\title{
LA GENÈSE
}

\section{L'ÉNERGIE PSYCHIQUE}

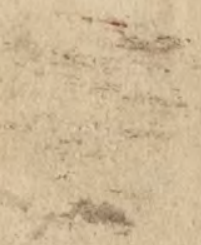

http://rcin.org.pl 


\section{OUVRAGES DU MEME AUTEUR}

Principes de l'évolution des Maladies infectieuses. 1918,1 volume in- 8 de 171 pages 5 fr.

Origine, évolution, traitement des Maladies non contagieuses. 1920, 1 volume in- 8 de 130 pages.......................... 


\section{ESSAI DE PHILOSOPHIE BIOLOGIQUE}

\section{LA GENESE}

DE

\section{LÉNERGIE PSYCHIOUE}

man

\section{J. DANYSZ -}

AVEC UNE LETTRE-PRÉFACE DE

\section{JULES PAYOT}

RECTEUR DE L'UNIVERSITÉ D'AIX-EN-PROVENCE

\section{PARIS}

LIBRAIRIE J.-B. BAILLIÈRE ET FILS 19, Rue Hautefeuille

http://rcin.org.pl 
Połaczone Biblioteki WFiS UW, IFIS PAN i PTF T.3963

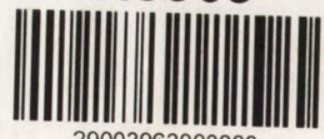

29003963000000

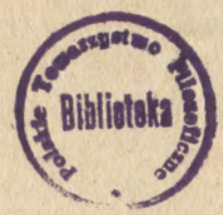

arreines. 524

http://rcin.org.pl 


\section{TABLE DES MATIÈRES}

Lettre-Préface de Jules Payot ............... $\mathrm{IX}$

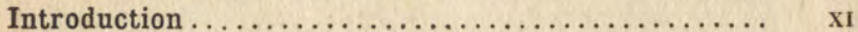

Livre Premier. - Evolution progressive de la matière et de l'énergie. De l'éther à l'homme. De l'attraction universelle à la pensée.

1. Nécessité de coordonner nos connaissances.... 1

2. Unités de matière et d'énergie. Synthèses successives ................... 7

3. Constitution des atomes.............. 9

4. Propriétés des atomes. Affinité chimique, moléculaire ...................... 11

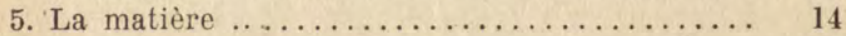

6. L'énergie ....................... 15

7. Unités " matière-énergie " ............. 18

8. Combinaisons chimiques. Les cristalloïdes.... 18

9. Les colloïdes $\ldots \ldots \ldots \ldots \ldots \ldots \ldots \ldots \ldots \ldots . \quad 19$

10. Micelles albuminoïdes. Multiplication. Accroissement infini. Différenciation........... 24

11. Substance cérébrale. Pensée............. 25

12. Tableau indiquant la succession des unités de matière et d'énergie................ 26

13. Lois de l'évolution des unités " matière-éner-

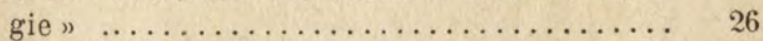

14. Le connu et l'inconnu................ 27

15. Constitution de l'univers. Formation des unités cosmiques $\ldots \ldots \ldots \ldots \ldots \ldots \ldots \ldots \ldots \ldots$ 
16. Influence de la température et de la pression sur les manifestations de l'affinité chimique.

17. Sélection par la décroissance progressive de la température et de la pression............

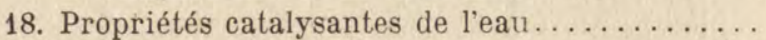

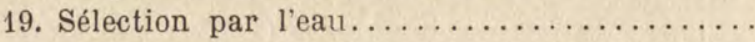

20. Milieu intramoléculaire et intramicellaire....

21. Formation des micelles organiques..........

22. Différenciation ................ 47

23. Cellules. Energie vitale. Tissus............

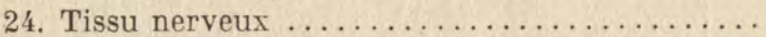

25. Energies rayonnantes. Le son: sa nature, sa formation, son aetion sur la matière vi-

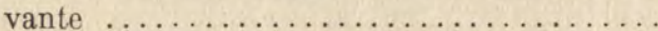

26. Intervention des énergies rayonnantes dans la formation des sens, de la mémoire, de la conscience et de la pensée..............

27. Comment expliquer l'absence des organes des sens chez les végétaux................

28. Evolution du tissu nerveux et des énergies ner-

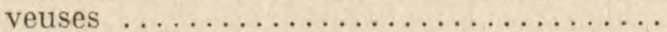

1. L'évolution progressive continue des vertébrés doit être cherchée dans le développement progressif du cerveau. - L'évolution du pallium..........

2. Développement parallèle du tissu nerveux et des énergies nerveuses......

3. L'immortalité .................. 77 88

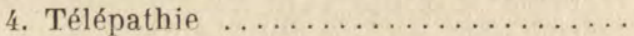

5. Résumé du paragraphe $28 \ldots \ldots \ldots \ldots$

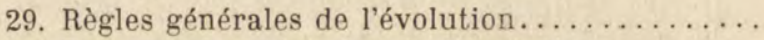

30. Les grandes périodes de l'évolution..........

31 . Le rôle de l'organisation des unités "matièreénergie” en associations de plus en plus complexes, dans, l'accroissement progressif de la puissance d'action de l'énergie....... 
32. Discontinuité et continuité de l'évolution. Rôle des catalyseurs. Eléments de structure et éléments de travail.................

33. Règles générales de l'évolution des milieux intérieurs. Sélection par l'intelligence. Température constante................... 34. Résumé et conclusions du Livre Premier......

Livre II. - Réactions de la matière vivante.

35. Evolution naturelle spontanée.............

36. Micelle albuminoïde vivante. Unité physiologique ..................... 125

37. Réactions provoquées par les cristalloïdes.... 136

38. Réactions provoquées par les colloïdes....... 151

1. Anaphylaxie ................ 153

2. Le rôle des colloïdes dans l'évolution.. 161

3. Résumé .................. 172

39. Rôle des énergies radiantes dans l'évolution progressive .................... 173

40. Rôle des énergies nerveuses dans l'évolution... 178

1. Réactions chimiques provoquées par les émotions ............... 178

2. Intervention des conceptions conscientes ................... 186

3. Résumé du paragraphe $40 \ldots \ldots \ldots \ldots . \quad 189$

41. Rôle des acides aminés et des vitamines...... 190

42. Résumé et conalusions du Livre II......... 198

Livre III. - L'évolution des êtres vivants, de l'individu et de l'espèce.

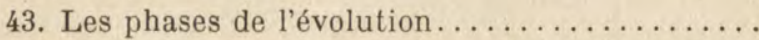

44. Evolution d'une plante annuelle et d'une plante vivace .................... 211

45. Multiplication des êtres unicellulaires: Infusoires ................... 217

46. Multiplication des microbes........... 219

47. L'individu et l'espèce................. 224

48. Formes de résistance. Conjugaison........ 227 
49. Accroissement continu ............... 230

50. Evolution de l'individu animal........... 232

51. Evolution des espèces animales........... 243

52. Filiation des espèces animales........... 247

53. Conditions de développement des cellules sexuelles ...................... 249

54. Les phases de l'évolution.............. 251

55. Fécondation ..................... 257

56. Evolution de l'oosperme. Division et différenciation ....................... 258

57. Relations entre l'individu et ses cellules sexuelles. Formation des cellules sexuelles.

58. Transmission de caractères nouveaux. Hérêdité et évolution................... 264 59. Résumé et conclusions du Livre III........ 267

Conclusions générales.

60. La place de l'homme dans la nature......... 271

61. Orientation actuelle de l'évolution............ 274

62. Classification des hommes suivant le degré de développement de leur encéphale et de leur énergie psychique ....................

63. Le rôle de la pédagogie dans l'éducation et dans

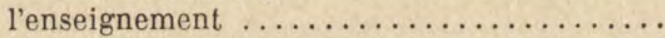

64. Tendance à une différenciation et à une sélection des individus d'une mentalité de plus en plus élevée. Réactions obligatoires et possi-

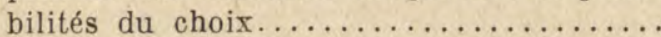
260

276 278 281

65. Classification des hommes suivant la nature des mobiles qui les dirigent dans leurs actions $\ldots \ldots \ldots \ldots \ldots \ldots \ldots \ldots \ldots \ldots \ldots \ldots \ldots \ldots \ldots \ldots \ldots \ldots \ldots$

66. L'évolution ne connaît pas de révolutions.... 285 291

67. Le chemin du bonheur plus grand pour le plus grand nombre 


\section{Mon cher Monsieur Danysz,}

Les quelques chapitres de votre nouveau livre qu'il m'a été donné de lire à Aix ont produit sur moi une double impression. Sur la première, j'aurais mauvaise grâce à insister: c'est celle d'une science profonde, d'une admirable prêcision, et où l'on devine votre vie tout entière consacrée, dans les laboratoires de l'Institut Pasteur, à la lutte patiente, persévérante pour conquérir un peu de cette vérité scientifique qu'on ne gagne qu'à la sueur de son front. Cette vérité est fuyante, mais, dans votre livre sur les Maladies chroniques non contagieuses, vous avez su saisir une découverte singulièrement bienfaisante et qui suffira, à elle seule, à immortaliser votre nom.

La seconde impression que j'ai éprouvée à la lecture de votre livre est analogue à celle que l'on éprouve en découvrant tout à coup, du haut d'une montagne, un vaste horizon...Si je vous ai bien compris, vous démontrez ce que tant de philosophes ont simplement affirmé: que l'univers tout entier tend vers la pensée. La matière si prodigieusement complexe a abouti à la substance cérébrale. Celle-ci s'est développée d'une façon éminente dans le cerveau humain. 
L'intelligence humaine s'est d'abord développée lentement durant l'immense pértode de la préhistoire. Ce développement est devenu considérable et rapide depuis que les inventions se sont multipliées et, à l'heure actuelle, tout nous permet de prévoir que la conquête de l'énergie spirituelle donnera des résultats merveilleux. L'homme actuel n'est qu'un stade dans cette conquête. Il sera dépassé, et les hommes dans l'avenir seront aussi supérieurs à l'homme actuel que celui-ci l'est à l'homme de l'époque des silex taillés. L'évolution intellectuelle, déjà rapide, le deviendra de plus en plus.

Quelles perspectives vous ouvrez sur l'avenir! Votre démonstration scientifique que l'évolution se fera vers une conquête de plus en plus haute de l'énergie spirituelle est saisissante; elle intéressera tous les philosophes et elle est de nature à renouveler entièrement la question si controversée de l'immortalité.

Ce livre vous fait grand honneur.

JuLES Payot. 


\section{INTRODUCTION}

En commençant ce livre j'avais un but précis : envisager sous un jour nouveau, et soumettre aux réflexions des lecteurs la question qui nous préoccupe tous, celle de la place de l'homme dans la nature et de son rôle dans les transformations continuelles de toutes choses, de son propre organisme et du milieu, dans lequel il vit, et fournir à ces réflexions les documents précis que la science expérimentale a mis à notre disposition jusqu'à ce jour.

Je me suis efforcé de démontrer que, malgré son origine commune avec tout autre être vivant, tant au point de vue de sa structure et de sa composition chimique qu'à celui de l'énergie qui l'anime, malgré qu'au point de vue purement biologique, l'individu homme, ou plus exactement l'ensemble de ses tissus somatiques (ce qui veut dire l'ensemble de l'organisme, excepté ses cellules sexueHles qui ne sont pas indispensables à la vie de l'individu), ne peut être considéré comme autre chose qu'un milieu de culture d'un être unicellulaire, qui se multiplie uniquement par simples divisions et conjugaisons de cellules libres et entièrement comparable, en cela, à un infusoire quelconque, en un mot, tout en reconnaissant comme incontestables les postulats essentiels des 
théories transformistes et évolutionnistes, on doit assigner à l'homme cultivé, tel qu'il est aujourd'hui, une place spéciale, unique, dans la nature.

L'homme est le premier être vivant qui, arrivé à un certain degré de développement cérébral et psychique, s'est demandé pourquoi il vit et, une fois qu'il s'est posé cette question, il n'a cessé d'y réfléchir et de chercher à y répondre.

Il a été amené ainsi à chercher à connaître son passé et le mécanisme de son présent et a éprouvé en même temps le besoin impérieux de prévoir son avenir, ou plutôt de s'en faire une idée.

Et rien n'est plus naturel que ce besoin de l'idée d'un avenir au delà du corps périssable, parce que, ayant conscience de sa supériorité psychique incontestable sur tous les autres êtres vivants et obligé de constater en même temps, que son corps était soumis aux mêmes besoins et sensations, que les corps des animaux qui, sous certains rapports, lui sont même supérieurs, l'homme ne pouvait se résigner à concevoir une existence sans autre but que la satisfaction d'occasionnels besoins immédiats et il a imaginé, aux différentes époques de son évolution, une genèse, un mécanisme vital et un avenir conformes au niveau de ses connaissances du mécanisme des réactions de son propre milieu, de son organisme, et du milieu extérieur.

C'est ainsi que, ne pouvant se faire aucune autre idée de l'origine de la vie, les premiers penseurs n'ont pu imaginer qu'une création et une ame immortelle qui, pour la durée de la vie de l'organisme devenait son énergie vitale et que ces simples vues d'esprit sont devenues des religions, c'est-àdire des professions de foi d'autant plus tenaces et 
farouches qu'elles étaient moins compréhensibles pour les foules auxquelles on les imposait.

Eh bien, ces théories devenues religions avaient le grave défaut, commun à toutes les religions que l'on a imposées à l'humanité jusqu'à présent, de fermer les portes sur l'inconnu, d'empêcher l'esprit de chercher à mieux comprendre, et s'il découvrait. un fait nouveau, d'imposer à la signification de ce fait, une interprétation convenue d'avance, et par cela même, inexacte.

Or, tout en reconnaissant que les théories sont nécessaires, nous savons qu'elles ne peuvent être que l'expression d'un ensemble de connaissances d'un moment, et loin d'en faire des professions de foi, nous en faisons des conceptions basées sur ces connaissances et destinées à rouvrir des portes nouvelles sur l'inconnu, au lieu de les fermer.

Une théorie sera toujours nécessairement une croyance parce que, tout en basant l'explication d'un phénomène sur un ensemble de faits connus, cet ensemble n'embrasse jamais tous les éléments qui concourent à ses manifestations, et cela serait-il possible, que l'explication d'un même phénomène variera avec la valeur que l'on donnera à chacun de ces éléments et sera différente pour les esprits différents.

Aussi, ne peut-il être question de chercher à enlever à l'humanité ses croyances, ses religions et ses illusions, mais il est dans l'intérêt d'une évolution progressive, d'en modifier les tendances, d'en faire des conceptions basées sur des ensembles de faits de mieux en mieux connus, des leviers du perfectionnement, non seulement de l'individu mais aussi de l'espèce. 
Le but de cet ouvrage est de montrer que ce perfectionnement ne peut être obtenu que par une évolution simultanée et équilibrée du corps et de l'esprit, d'indiquer par quel mécanisme on peut l'obtenir et quelles peuvent en être les limites; de montrer que, depuis qu'il en a eu conscience, l'homme est devenu capable de faire prendre à cette évolution, par des actes volontaires, une direction déterminée, qu'il sait déjà sur quels organes ou tissus de son organisme il faut agir et qu'il apprendra peu à peu comment il faut agir pour rendre cette évolution plus sûre et plus rapide, qu'en un mot il trouvera "le pourquoi" de sa vie dans l'idéal d'un avenir illimité de son espèce, dans sa croyance à la toute-puissance de l'homme futur.

Le développement de nos connaissances, de notre conscience en étendue et en délicatesse de perception, se faisait toujours et se fait encore en procédant du plus simple au plus complexe. Tout phénomène se présente à nous, tout d'abord par l'effet global qu'il produit, comme une entité simple, indivisible. Les phénomènes connus de cette façon peuvent être coordonnés en un ensemble qui parait logique à un moment donné. Le fait que le soleil tourne autour de la terre, et que c'est un Dieu qui se lève le matin et se couche le soir, était aussi évident et aussi bien adapté à l'état d'esprit de nos ancêtres, que les lois astronomiques établies par Kopernik, Newton, Einstein, le sont pour notre esprit d'aujourd'hui.

Mais, faut-il en conclure qu'il en sera toujours ainsi, qu'en changeant d'idée au sujet de la valeur respective et de la coordination des phénomènes nous n'arriverons qu'à changer une erreur en une autre erreur qui semblera à nos successeurs tout 
aussi peu justifiée, quoique peut-être d'une naïveté moins poétique?

Certainement non! Nous avons appris que pour apprécier la valeur d'un phénomène il faut connaître les éléments dont il se compose, le mécanisme de chacun de ces éléments, ainsi que leurs interactions; nous avons appris à le reproduire et souvent à le modifier à volonté ou à l'adapter à nos besoins. Nos théories ne sont plus basées uniquement sur des idées préconçues, mais sur des faits constatés par l'expérience, sur la conception de la valeur relative de ces faits que nous cherchons à classer suivant l'importance de leur rôle dans le mécanisme de chaque phénomène et, si nous prévoyons qu'elles peuvent être modifiées dans l'avenir, nous savons aussi, que ces théories appuyées sur des faits bien étudiés, dont elles expliquent le mécanisme, indiquent en même temps ce qu'il reste à chercher et provoquent ainsi des travaux qui nous permettent de faire quelques pas de plus dans l'intimité, dans la connaissance profonde du mécanisme et du sens général, de la raison d'être des phénomènes.

Pour rendre l'exposé qui suit, aussi clair que possible, j'ai suivi ce chemin à rebours. En effet, pour expliquer la formation matérielle et énergétique de l'homme actuel ainsi que le mécanisme de ses fonctions, et indiquer quel peut être son avenir, il m'a semblé indispensable de retracer les grandes lignes de l'évolution de la matière inorganique dont il est composé, en commençant par les unités les plus simples, actuellement connues, les électrons, - peut-être les éthérons, - de montrer ensuite comment la matière inorganique a pu devenir vivante, comment l'être vivant le plus simple, la 
cellule, a pu devenir homme, et enfin quel peut être l'avenir de l'homme.

Je suis particulièrement heureux de pouvoir exprimer ici mes sincères remerciements à mes collaborateurs et collaboratrices et plus spécialement à Mmes Michel-Danysz, Desmoulins-Simonneau et mon fils Stéphane, qui ont bien voulu m'aider dans mes recherches et mes expériences, ainsi que de témoigner mes sentiments de considération et de cordiale amitié à J.-G. Haller, qui a été pour moi le modèle de l'homme d'action de l'avenir, qui, par son éducation philosophique, saura concilier les nécessités de la vie individuelle avec le progrès général de l'humanité. 


\title{
LA GENÈSE \\ DE L'ÉNERGIE PSYCHIQUE
}

\author{
LIVRE PREMIER
}

\section{ÉVOLUTION DE LA MATIÈRE ET DE L'ÉNERGIE DE L'ÉTHER A L'HOMME} DE L'ATTRACTION UNIVERSELLE A LA PENSÉE

\section{1. - Nécessité de coordonner nos connaissances}

Les organismes vivants les plus complexes sont composés d'éléments que l'on retrouve dans la nature inerte. Un microbe ou un infusoire, qui vit dans un bouillon de culture et qui se multiplie aux dépens des substances inorganiques qui s'y trouvent, ne peut construire ses tissus qu'exclusivement avec les matières minérales qu'il trouve dans son milieu, et les réactions chimiques que ces substances subissent spontanément dans les tissus vivants ne diffèrent en rien de celles que nous pouvons réaliser avec les mêmes substances dans nos laboratoires.

La seule différence qui existe entre les éléments qui composent le milieu et ces mêmes éléments qui composent les corps de la cellule vivante, c'est que,

Danysz. - La genèse de l'énergie psychique. 
dans le milieu les éléments sont inertes les uns pour les autres, c'est-à-dire s'y trouvent dans un équilibre très durable, sinon absolument stable et qui ne peut être modifié que par des énergies extérieures, telles que la chaleur, la lumière, l'électricité ou les réactifs chimiques, tandis. que dans la cellule, il s'établit entre ces mêmes éléments un équilibre dynamique, très instable dans les réactions de détail, mais très stable dans l'ensemble. Les éléments d'une cellule vivante subissent une série de réactions de synthèse et de décomposition, qui se succèdent d'une façon incessante et toujours dans le même ordre, et qui s'effectuent spontanément, c'est-à-dire sans intervention d'autres énergies que celles que la cellule trouve à l'état potentiel dans les éléments de son milieu intérieur.

Mais, si nous savons que les choses se passent ainsi, si nous en connaissons le mécanisme et pouvons même entrevoir la source de l'énergie qui fait travailler la cellule, - qui, en somme, ne fonctionne pas autrement qu'une machine à vapeur, en brûlant du carbone -, il nous faut chercher encore par quelle série de formations ou d'évolutions partielles, matérielles et énergétiques, la matière inerte est devenue vivante; ou, en d'autres termes, d'où est venue, comment s'est formée l'énergie qui puise dans le milieu extérieur, transporte dans le foyer de la chaudière et allume le charbon nécessaire pour faire travailler la machine.

Il ne faut pas se faire d'illusions. Il nous sera encore impossible de préciser tous les détails de ce problème, mais il n'en est pas moins intéressant de synthétiser l'ensemble de nos connaissances à ce sujet, de faire ressortir quelques lois ou règles géné- 
rales qui régissent l'évolution des unités successives de la matière et des formes d'énergie, parce que, seule, la connaissance de ces règles générales nous permettra de classer les faits d'observation et d'expérience suivant leur importance, de leur donner leur véritable signification, d'ouvrir de nouveaux horizons pour des recherches nouvelles. Ce travail, cette sorte d'examen de eonscience scientifique nous semble aussi nécessairement obligatoire dans l'évolution de nos connaissances que la succession de synthèses dans l'évolution des unités de matière. II forme une étape, un échelon indispensable sur lequel on peut s'appuyer pour monter plus haut à l'échelle du progrès, une étape sans laquelle aucun travail bien organisé n'est possible.

L'homme est un produit de la nature. L'homme vivant est un assemblage de matière et d'énergie; dans sa formation et son évolution il subit les lois générales qui régissent l'évolution de toute autre unité de matière et de toute autre forme d'énergie, mais il est la seule unité de l'évolution qui possède le privilège de la forme d'énergie consciente d'ellemême. Il constate sa propre existence, ainsi que l'existence de toutes choses autour de lui et, ayant éprouvé le besoin de connaître le "comment " et le "pourquoi " de toutes choses, il arrive peu à peu à modifier le cours des phénomènes naturels spontanés, à les adapter à ce qu'il croit lui être utile ou agréable, à son individu et à son espèce.

Et l'évolution du savoir passe toujours par les trois étapes successives : l'observation des choses et des phénomènes dont la conscience constate l'existence par l'intermédiaire des organes des sens, la recherche du mécanisme des phénomènes, dont l'analyse 
confirmée par l'expérience nous apprend "comment " ils se produisent et enfin l'interprétation du " pourquoi » des phénomènes, la recherche des relations qui peuvent exister entre eux, de leur raison d'être, de l'importance de leur rôle dans l'ensemble des phénomènes naturels.

Ces trois stades de l'évolution de la connaissance correspondent à trois sortes de réactions de l'organisme : purement réflexe ou automatique, instinctive et raisonnée, et, ainsi que nous le verrons plus loin, dans le chapitre consacré à l'évolution du tissu nerveux, ces réactions ont leur origine et leur siège dans trois sortes d'organes, de connexions et de centres nerveux, qui naissent successivement les uns des autres.

Et quand la conscience est parvenue au plus haut degré de son évolution actuelle, quand elle a éprouvé le besoin de comprendre le sens des phénomènes, leurs connexions, relations et réactions réciproques, leur rôle dans l'évolution générale, elle a été obligée de reeonnaître que la connaissance d'un phénomène de son "comment" et de son "pourquoi " ne peut être complète, qu'à la condition d'en trouver l'origine, le passé et, autant que c'est posșible, d'en prévoir lavenir.

Ce sont toujours les manifestations énergétiques qui nous frappent les premières. Nous sommes amenés à les étudier en elles-mêmes, comme des entités indépendantes de la matière, c'est ensuite seulement que la réalité des choses nous oblige à en ehercher l'origine dans la matière dont elles émanent.

L'homme a eu conscience de sa pensée avant de connaître son cerveau. Il apprend peu à peu à eonnaître son corps, comme il apprend à connaître 
le monde extérieur et c'est là la raison de la conception d'une énergie psychique, d'une "âme" complètement indépendante du corps. Il a fallu de longues recherches pour constater que l'âme a subi une évolution progressive; que telle que nous la connaissons aujourd'hui chez l'homme civilisé, elle est formée de qualités et de facultés qui sont apparues successivement à mesure que se développait le tissu nerveux chez lui et avant lui chez les autres êtres vivants, qu'elle a donc eu pour origine la sensibilité nerveuse, que cette dernière n'est que le résultat d'une différenciation et d'une localisation de la sensibilité générale de la matière vivante qui, à son tour, doit cette propriété à l'affinité chimique et aux formes d'énergie physique dont est douée la matière minérale.

Pour trouver l'origine de la pensée qui est aujourd'hui l'apanage exclusif du cerveau humain, il faut donc descendre peu à peu jusqu'à la forme d'ênergie la plus simple : l'attraction universelle, - ainsi qu'à tous les échelons formés par les unités de matière de plus en plus simples : les micelles, les molécules, les atomes, jusqu'à l'éthéron qui est à la limite de ce que nous pouvons concevoir de plus simple actuellement.

Avec les unités matérielles et les formes d'énergie de plus en plus simples ou complexes on peut done former aujourd'hui une chaîne ininterrompue depuis l'éthéron jusqu'à l'homme et depuis l'attraction universelle jusqu'à la pensée, une chaîne dans laquelle les chaînons: "unités de matière ", et "formes d'énergie » se commandent réciproquement.

L'étude de chacun de ces chaînons est aujourd'hui l'objet d'une ou de plusieurs grandes branches de 
sciences spéciales: physique, chimie, chimie physique, astronomie, toutes les branches de la biologie, en un mot de tout notre savoir. Notre but, en entreprenant cette étude, ne peut donc pas être de donner ici un résumé de toutes ces sciences, mais simplement d'en prendre les notions exactes qui nous semblent utiles pour montrer l'existence d'une évolution progressive dans l'univers et l'enchaînement nécessaire des formations successives, de chercher à expliquer comment les choses ont pu se passer et, autant que cela sera possible, pourquoi il devait en être ainsi.

Ceci fait, nous ehercherons à nous expliquer, avec un peu plus de détails, les propriétés fondamentales et les réactions caractéristiques de la matière vivante, ainsi que l'évolution des individus vivants et des espèces et notamment les raisons du "pourquoi " de l'évolution cyclique de l'individu, de sa mort nécessaire et de la durée, théoriquement infinie de l'espèce.

La matière vivante est formée des éléments de la matière minérale. Les réactions chimiques de la matière vivante ne sont autres et ne peuvent être autres que, celles des éléments inorganiques; mais à chaque étape de synthèse de la matière surgissent des formes d'énergie nouvelles. Aussi, pour apprécier les propriétés des organismes vivants, comprendre le mécanisme de leurs fonctions, établir les règles de leur évolution, faut-il avant tout établir ce que nous savons de l'évolution des unités "matière-énergie " de la matière inorganique. Il nous serait impossible d'entrer ici dans tous les détails de cette étude, et ce n'est d'ailleurs pas nécessaire. Il nous suffira d'indiquer les règles générales de 
l'évolution des unités "matière-énergie " de la matière minérale pour nous faire ensuite une idée suffisamment claire de l'évolution des êtres vivants.

\section{2. - Unités de matière et d'énergie. Synthèses successives}

L'étude de toutes les choses qui nous entourent et des pénomènes dont nous sommes les témoins ou les agents nous a conduit à constater que les unités matérielles distinctes qui ont apparu successivement à la surface de la terre ont pour origine commune l'unique matière primordiale : l'éther des physiciens et l'énergie universelle : le mouvement.

Ainsi, nous avons appris peu à peu que l'homme, de même que tous les organismes multicellulaires, ont eu pour origine des êtres unicellulaires et qu'ils sont encore aujourd'hui les produits de la multiplieation d'une seule cellule; que ces cellules primordiales résultent de la combinaison des molécules d'un certain nombre d'éléments simples ;

que ces molécules sont formées de la réunion d'un certain nombre d'atomes composés, formés euxmêmes de l'assemblage d'un plus ou moins grand nombre d'atomes simples et ces derniers d'électrons;

et enfin qu'au delà des électrons, il y a l'éther, c'està-dire quelque chose qui échappe complètement à nos moyens actuels d'appréciation, mais dont nous sommes obligés d'admettre l'existence, parce que seule l'existence de l'éther nous permet d'expliquer la propagation dans l'espace des rayons ultra-violets, lumineux, caloriques et électriques et autres énergies rayonnantes.

Ce sont les vibrations, c'est-à-dire certains mouve- 
ments coordonnés de l'éther qui se traduisent pour nos sens ou nos instruments de mesure, sous forme d'énergie ultra-violette, lumineuse, calorique ou électrique, et comme il nous est absolument impossible de concevoir un mouvement autrement que comme un corps, une unité matérielle en mouvement, nous sommes bien obligés d'admettre que, tout en ne possédant aucun moyen de les apprécier de quelque façon que ce soit, l'éther doit être eomposé d'unités distinctes, "d'éthérons ", de même que l'eau ou l'air sont composés de molécules distinctes.

L'unité d'éther serait donc l'unité de matière la plus petite et la plus simple que nous puissions concevoir aujourd'hui et, sur notre planète, l'homme est actuellement l'unité de matière la plus complexe, par conséquent, on pourrait dire en dernière analyse que l'unité de matière " homme » est de l'éther condensé.

Mais là il faut s'entendre ! La condensation pure et simple des unités d'éther ne donnera jamais un homme, quel que soit le nombre de ces unités réunies ensemble. Pour former cette unité, la plus complexe de matière et d'énergie, il à fallu une longue évolution, dont les étapes sont marquées par l'apparition successive des électrons, des atomes, des molécules composées, des micelles et enfin des cellules vivantes, c'est-à-dire d'unités de matière et d'énergie dont la complexité plus grande à chaque degré résulte de la réunion en un ensemble distinct d'unités d'ordres inférieurs plus simples. Ainsi, les unités d'éther, en se condensant, ne peuvent former que des électrons. Les unités d'éther sont bien probablement toutes identiques entre elles, mais elles peuvent se grouper de deux façons différentes et en nombres différents puisqu'on admet l'existence de deux sortes 
d'électrons: les uns sont des charges électriques positives, les autres négatives.

La forme d'énergie "électricité " n'existe probablement pas dans un milieu composé uniquement d'unités d'éther. On constate ses manifestations dans les électrons et on ne peut pas se la représenter autrement que comme une résultante de la différence de structure et des mouvements, des électrons positifs et négatifs réagissant les uns sur les autres.

Et de même que c'est l'énergie propre des unités d'éther qui détermine le groupement de ces unités de premier ordre en unités du deuxième ordre, ou électrons positifs et négatifs, c'est l'électricité qui détermine le groupement des électrons en unités du troisième ordre, ou atomes simples dont on commence à entrevoir la constitution.

\section{3. - Constitution des atomes}

Suivant la théorie indiquée par Crookes, formulée il y a une vingtaine d'années par J.-J. Thomson, confirmée et complétée ensuite par Van den Broeck, Rutherford et par Bohr, on doit se représenter l'atome comme un ensemble analogue dans sa structure et dans ses mouvements à notre système solaire, c'està-dire formé d'un noyau central composé d'électrons positifs et d'un nombre plus ou moins grand d'électrons négatifs qui tournent autour de ce noyau, comme les planètes tournent autour du soleil.

Van den Brock et Rutherford ont établi par l'étude de certaines propriétés des corps radio-actifs, que le nombre d'électrons négatifs d'un atome est égal à ce qu'on appelle le "nombre atomique", celui des électrons positifs, au "poids atomique». Pour un 
atome d'hydrogène, l'élément le plus léger, ce serait un électron positif et un négatif; pour un atome d'uranium, l'élément le plus lourd, 238 électrons positifs et 92 négatifs.

Nous ne savons pas combien d'espèces d'atomes différents se sont formés par la réunion des électrons, parce que nous ne connaissons qu'un petit nombre d'éléments monoatomiques. Ce sont, par exemple, l'hélium, le crypton, le néon, que l'on trouve à l'état de gaz dans l'air et que l'on a appelés gaz inertes, privés d'affinité, parce que, dans les conditions dans lesquelles on a pu les étudier jusqu'à présent, on n'a pu les faire entrer dans aucune combinaison eonnue.

Nous ne savons pas non plus par quelle forme d'énergie les atomes simples ont été réuniś en atomes composés. Pour Soddy, un atome composé ne résulte pas d'une combinaison d'un certain nombre d'électrons et d'atomes simples, ce n'est pas une combinaison chimique comparable à un sel formé d'un plus ou moins grand nombre d'atomes composés, mais on a constaté avec certitude que l'atome d'uranium I (238) devient successivement de l'uranium $x_{1}, x_{2}$, II (234) de l'ionium (230), du radium (226), de l'émanation (222), du radium $\mathrm{A}(218)$, du radium $\mathrm{B}, \mathrm{C}, \mathrm{C}^{\prime}$ (214), du radium $\mathrm{C}_{2}, \mathrm{D}, \mathrm{E}, \mathrm{F}$ (210) et enfin du radium $\mathrm{G}$ ou du plomb (206) en perdant chaque fois un atome d'hélium (4), que, par conséquent, la molécule d'uranium I contient au moins neuf atomes d'hélium (1). On peut en déduire que les atomes de tous les autres

(1) La complexité des atomes pourrait faire admettre aussi que les atomes d'hélium ne se trouvent pas tels quels dans la molécule de l'uranium, mais qu'ils se forment par la décomposition de ce dernier. 
éléments sont formés par l'assemblage d'un nombre plus ou moins grand d'atomes simples.

On peut donc affirmer que les unités de $3^{e}$ ordre, les atomes "s'assemblent " pour former des unités de $4^{\circ}$ ordre; les atomes composés dont on connaît aujourd'hui 86 espèces différentes et dans lesquelles apparaît une forme d'énergie nouvelle: l'affinité chimique ou moléculaire.

\section{4.-Propriété des atomes. Affinité chimique moléculaire}

L'éther, les électrons et les atomes sont done les premiers échelons de l'évolution de la matière. L'étude de la formation et des propriétés de ces premières unités de la matière est du domaine exclusif de la physique, parce qu'on ne leur connait aucune propriété chimique.

Avec l'apparition des atomes composés commence une nouvelle étape de cette évolution ascendante qui nous mènera jusqu'à l'homme et dont le facteur initial est l'affinité chimique. L'étude des combinaisons entre atomes appartiendra désormais à la chimie, sans cesser toutefois d'avoir recours à la physique, parce que, à côté des propriétés chimiques des unités que les combinaisons auront formées, nous aurons à considérer leurs structures.

Qu'est-ce qu'un atome composé?

Qu'est-ce que l'affinité chimique?

Au point de vue de leur structure, nous savons que les atomes composés sont formés de plusieurs atomes simples, de sorte qu'en admettant la théorie de Thomson, pour la structure de l'atome, les atomes composés seraient des systèmes de plusieurs sys- 
tèmes solaires en mouvement, maintenus ensemble par un noyau réel ou un centre d'attraction idéal.

Comment les atomes sont-ils rangés autour de ce centre d'attraction et quels sont leurs mouvements, le sens et la vitesse de ces mouvements ? Décriventils des orbites circulaires ou elliptiques, ou sont-ils animés de mouvements de va et vient? Nous ne le savons pas; mais ce que nous pouvons admettre avec certitude, c'est que l'ensemble qui forme l'atome ainsi que toutes les unités de tous les ordres qui le composent sont animés de mouvements incessants et que c'est du concours de tous ces mouvements, de toutes ces formes d'énergie, que résulte l'énergie d'ensemble de l'atome, l'affinité chimique.

Cest en vertu de cette nouvelle forme d'énergie que les atomes composés se combinent entre eux pour former des molécules, c'est-à-dire tous les corps liquides et solides qui constituent la croûte terrestre et tous ceux que nous savons préparer dans nos laboratoires, et l'étude des propriétés chimiques de ces corps nous a appris que l'affinité est une forme d'énergie qui résulte des différences des propriétés physiques des atomes, d'une part, de leur structure qui donne la valence de la molécule, d'autre part, du sens et de la vitesse des mouvements de l'ensemble de la molécule, ainsi que de tous ses composants à tous les degrés: des atomes simples, des électrons dans les atomes et des étherons dans les électrons, qui déterminent le choix ou l'électivité des attractions.

Les différences des charges électriques jouent un rôle important dans les manifestations de l'affinité, mais ne l'expliquent pas entièrement. Entre autres, elles n'expliquent pas ce fait, que pour chaque couple d'éléments qui peuvent se combiner, il faut des con- 
ditions de milieu et, notamment, de température et de pression différentes pour que l'affinité puisse so manifester, alors que dans d'autres conditions de milieu, les atomes de ces mêmes éléments resteront indifférents, et que dans certains autres encore ils vont se repousser.

Il faut noter aussi que l'affinité ne s'exerce pas à distance; elle ne rayonne pas dans l'espace, ne provoque aucune manifestation appréciable dans le milieu extérieur des molécules, comme l'électricité, la lumière ou le son. On peut dire que c'est une forme d'énergie qui n'apparaît pas et ne peut se manifester que dans ou par le milieu intérieur des atomes. Pour se combiner, deux atomes doivent "s'accrocher", pénétrer dans les zones d'influences intimes réciproques.

L'étude de ces conditions dans lesquelles se manifeste l'affinité entre deux éléments, dans lesquelles deux atomes différents "s'accrochent " l'un à l'autre et restent accrochés en perdant toutes leurs propriétés individuelles pour les retrouver quand ils redeviennent libres, est donc une des plus intéressantes de la chimie physique, une des plus importantes aussi, parce qu'elle nous permettra d'entrevoir l'action des catalyseurs et, par là, le passage tout naturel de la matière dite "inerte" à la matière vivante.

Toutefois, les notions que nous cherchons à résumer ici forment un ensemble tellement complexe que, pour les coordonner et les présenter d'une façon aussi claire que possible, il nous faut d'abord essayer de définir les principaux éléments de l'évolution progressive. 


\section{5. - La matière}

On peut appeler matière la somme de toutes les unités contenues dans l'espace, dont il est possible de mesurer ou de concevoir un volume et un poids.

Suivant le degré de leur évolution qui dépend d'un ensemble de conditions du milieu dans lequel elles se trouvent à un moment donné, ces unités sont plus ou moins complexes. Théoriquement, on ne peut pas imaginer une unité de matière qui ne serait pas formée de la réunion d'unités plus petites et plus simples. Chaque unité de matière serait donc infiniment divisible et devrait pouvoir former, par réunion ou combinaison avec d'autres unités du même ordre ou avec des unités de l'ordre inférieur, des unités de plus en plus complexes. Théoriquement, l'évolution ascendante ou la synthèse, de même que l'évolution descendante ou la décomposition, ne devrait donc pas avoir de limites.

Pratiquement, au point de vue de l'évolution prógressive, la première unité, la plus petite et la plus simple dont nous puissions apprécier le volume et le poids est l'électron (1); la dernière, sur notre planète, est actuellement l'homme.

Entre l'électron et l'homme, on peut distinguer sept grandes étapes ou degrés de synthèse caractérisées par la formation dans l'ordre ascendant des atomes simples, des atomes composés ou des molécules simples, des complexes ou micelles, des cel-

(1) On est pourtant obligé d'admettre l'existence d'un milieu formé d'unités encore inflniment plus petites que les électrons, e'est l'éther des physiciens qu'il n'est guère possible d'imaginer autrement que formé d'unités distinctes et que l'on pourrait appeler éthérons. 
lules, des tissus et enfin de la substance nerveuse et cérébrale.

\section{6. - Energie}

L'énergie c'est le mouvement; et, ainsi que nous l'avons indiqué plus haut, il est impossible de se représenter un mouvement d'une façon autre, que comme le déplacement d'une unité matérielle. Toutes les formes d'énergie que nous connaissons ne peuvent être que les effets ou les résultantes de mouvements des unités matérielles simples ou plus ou moins complexes.

Suivant la nature des unités matérielles et de leurs mouvements d'ensemble ou de leurs mouvements intérieurs, on peut classer les énergies en trois catégories différentes:

$1^{\circ}$ Les formes d'énergie produites par les mouvements rythmiques sur place de certaines unités de matière;

$2^{\circ}$ Celles qui sont produites par le déplacement dans l'espace ou par des projections d'unités matérielles;

$3^{\circ}$ Celles qui résultent d'un ensemble de mouvements combinés d'un certain nombre d'unités matérielles réunies en unités distinctes, comme par exemple les mouvements du soleil, des planètes et de leurs satellites dans un système solaire dont l'ensemble forme une unité distincte.

Dans la première catégorie de formes d'énergie que nous connaissons actuellement, doivent êtré rangées les énergies rayonnantes ou rythmiques, à savoir : les rayons $\mathbf{X}, \gamma$, ultra-violets, visibles, caloriques et 
électriques, provoqués par les mouvements rythmiques des électrons qui font vibrer l'éther, et le son provoqué par les mouvements rythmiques des molécules qui font vibrer l'air ou toute autre substance sólide, liquide ou gazeuse composée de molécules.

Les formes d'énergie qui résultent du mouvement de projection, de déplacement des unités matérielles dans l'espace, sont les rayons $\alpha$ (atomes d'hélium) et les rayons $\beta$ (électrons négatifs), et je crois que l'on doit ranger dans la même catégorie: l'odeur qui résulte de certains mouvements d'ensemble de certaines molécules simples ou composées sous forme de gaz ou de vapeur, et la saveur produite par certains mouvements de molécules simples, composées ou complexes, sous forme liquide.

C'est probablement grâce aux répulsions réciproques que les molécules des gaz, ainsi que les molécules en suspension dans l'eau, occupent, dans certaines limites, tout l'espace qu'elles ont à leur disposition.

Les formes d'énergie de la troisième catégorie, celles qui résultent du concours d'un certain nombre d'unités matérielles en mouvement, réunies en unités distinctes d'un ordre supérieur, sont fonction, dans leurs manifestations, de la composition, de la structure et des mouvements intérieurs, c'est-à-dire de l'ensemble des conditions du milieu intérieur des unités de matière qui les produisent. Ces énergies ne rayonnent pas dans l'espace et ne sont donc pas directement transmissibles. Ce sont ces énergies du milieu intérieur qui sont appelées à exécuter, échelon par échelon, le travail de construction des unités de plus en plus complexes.

Ce sont l'affinité chimique, l'affinité colloïdale ou 
micellaire, la sensibilité et enfin la conscience et la pensée.

De par leur formation par la constitution du milieu intérieur, ainsi que du fait qu'elles ne sont pas rayonnantes, ces énergies ne peuvent ni augmenter, ni diminuer, ni se transformer en énergie d'autres formes. La quantité de lủmière ou de chaleur qu'un corps quelconque possède à un moment donné sera constamment diminuée avec le temps, par rayonnement dans l'espace et ne pourra être retrouvée, que par un apport nouveau de ces énergies du milieu extérieur, tandis qu'un élément quelconque possédera toujours la même quantité d'affinité chimique, la même force de combinaison, dans les mêmes conditions de milieu (1). Mais, ainsi que nous le verrons plus loin, en parlant de la nature de l'affinité chimique, la manifestation de ces énergies, leur plus ou moins de force et d'activité dépendent de l'état dans lequel se trouve l'unité qui la produit, ainsi que des conditions de milieu extérieur qui peuvent modifier cet état.

Ainsi, les diverses formes d'énergie ne peuvent être que les manifestations des mouvements de diverses unités matérielles, agissant, soit par l'ensemble de leurs masses, soit par une coordination des mouvements de leurs composants (2).

(1) Excepté les corps radioactifs. L'unanium, par exemple, est' un corps chimiquement différent du radium.

(2) D'après les physico-mathématiciens modernes (Lorenz, Langevin, etc.), les électrons, dont sont formés les atomes, ne seraient pas matériels. Ce seraient des unités d'énergie électromagnétique toute seule, sans aucun support matériel. C'est une conception mathématique dont nous n'avons pas à nous occuper ici.

Daxrsz. - La genèse de l'énergie psychique. 


\section{7. - Unités " matière-énergie »}

En réalité, l'univers n'est pas composé de matière et d'énergie, mais uniquement d'unités de matière en mouvement ou, en d'autres termes, d'unités matièreénergie, dont les manifestations de plus en plus complexes résultent d'une série de synthèses successives.

Nous ne connaissons pas les formes d'énergie qui déterminent la formation de électrons. C'est peut-être l'attraction universelle?

Nous ne pouvons pas affirmer non plus avec certitude si les électrons sont formés de la réunion des éthérons, mais on peut admettre que la réunion des électrons en atomes est déterminée par la forme d'énergie électricité qui naît de la différence de structure entre les électrons positifs et négatifs, si tant est qu'il y a une électrieité positive.

Nous ne savons pas encore si les atomes élémentaires, que l'on peut appeler aussi atomes composés $(\mathrm{O}, \mathrm{H}, \mathrm{Na}, \mathrm{Cl}$, ete.) pour les distinguer des molécules composés $\left(\mathrm{H}^{2} \mathrm{O}, \mathrm{NaCl}\right.$, etc.), sont formés directement de la réunion des électrons, ou de la réunion d'atomes simples.

$\mathrm{Si}$, entre les électrons et les atomes composés, la formation intermédiaire des atomes était nécessaire, il faudrait encore chercher la forme d'énergie qui réunit les atomes simples en atomes composés, parce que, autant qu'il est possible d'en juger, cette énergie n'est pas l'affinité ehimique.

\section{8. - Combinaisons chimiques. Les eristalloïdes}

La réunion des électrons ou des atomes, ou encore des uns et des autres en atomes composés a eu pour 
résultat, ainsi que nous l'avons vu, la naissance d'une énergie nouvelle, l'affinité chimique, qui ne se manifeste et n'existe pas dans les unités d'ordre inférieur. C'est grâce à l'affinité ehimique que les atomes composés, au nombre de 86 , se combinent entre eux, dans certaines conditions, en molécules: acides, bases et sels, plus ou moins complexes.

Les réactions de combinaison entre les acides, les bases et les sels sont toujours précédés d'une dissociation des moléeules composées en atomes, de sorte que les réactions s'effectuent, en réalité, entre atomes, et le seul facteur de ces réactions est toujours l'affinité chimique, que l'on pourrait appeler aussi affinité atomique ou moléculaire.

\section{9. - Les colloïdes}

Les colloïdes diffèrent des cristalloïdes par un ensemble de propriétés chimiques et physiques.

Au point de vue chimique, une moléeule est un composé défini de deux ou plusieurs atomes qui se combinent entre eux toujours dans les mêmes proportions, suivant leurs valences, et donnent toujours des produits identiques, même si la technique opératoire, concentration des liquides, etc., n'est pas toujours la même.

Les micelles, au contraire, ne sont jamais des corps purs dans le sens que l'on altribue à ce mot en chimie cristalloïde, et les proportions des éléments qui les composent peuvent varier dans de larges proportions.

Ainsi dans l'hydrate chloroferrique $\left(\mathrm{Fe}^{2} \mathrm{Cl}^{6}, \mathrm{n} \mathrm{Fe}^{2}\right.$ $\left(\mathrm{OH}^{6}\right)$, la valeur de $n$ peut varier de 5 à 800 , c'est-àdire que pour une molécule de chlorure il y aura 
5 à 800 molécules d'hydrate, ce sera toujours un liquide brun ou rouge foncé, mais ses autres propriétés physiques varieront autant que les proportions de deux composants. Relativement très stable, tant que la valeur de $n$ ne dépassera pas 20, le composé le deviendra de moins en moins quand la valeur de $n$ augmentera.

Toutefois, pour se rapprocher autant que possible des réactions que peuvent subir les colloïdes qui composent la matière vivante, on doit se représenter les variations des micelles d'une façon différente.

Une micelle est toujours composée d'une partie inactive et d'une autre, électriquement active. Dans l'exemple que nous avons choisi, la partie inactive c'est l'hydrate ferrique, la partie active le chlorure. Eh bien, en réalité, les propriétés de l'hydrate ne peuvent varier que si les proportions du chlorure varient.

$\mathrm{Si}$, par exemple, il y a dans un liquide 100 molécules d'hydrate pour 20 molécules de chlorure, il y aura un groupe de 5 molécules d'hydrate réunies en une seule particule pour une molécule de chlorure. $\mathrm{Si}$ alors on enlève 10 molécules de chlorure (par dialyse), les particules d'hydrate se réuniront deux par deux et chaque particule composée de 10 molécules d'hydrate sera tenue en équilibre par une molécule de chlorure; si on enlevait encore 9 molécules de chlorure, pour n'en laisser qu'une, toutes les 100 molécules d'hydrate se réuniraient en une seule masse qui serait tenue en équilibre par la seule molécule de chlorure qui reste, mais cet équilibre sera bien plus instable que si la proportion était de 1 à 5 .

Ainsi, le volume et la précipitabilité de la partie inactive de la micelle, mais qui cependant donne son caractère chimique au colloïde, augmente à mesure 
que la quantité des molécules de la partie active diminue et c'est là une notion de la plus haute importance pour la compréhension des réactions de la matière vivante, composée, comme on sait, de cellules qui sont autant de dialyseurs.

La partie active est généralement un sel ou un acide facilement dialysable; la partie inactive peut dialyser ou non, suivant la grosseur et le nombre des molécules dont elle est composée. Généralement, dans une cellule vivante, elle n'est pas dialysable et reste constamment à cet état intermédiaire entre le solide et le liquide (entre le gel et le sol) appelé précisément colloïde, qui explique la facilité des échanges entre la cellule et son milieu extérieur, toujourś liquide.

Il y a des colloïdes qui, une fois solidifiés, ne se redissolvent plus, et d'autres, pour lesquels ces réactions sont réversibles. Cela dépend de la composition chimique et de la structure de la partie inactive des micelles et explique la formation des parties plus ou moins stables ou instables de la cellule (membrane, plasma, noyaux, etc....).

$\mathrm{Si}$, à ces notions on ajoute encore celle de fixation élective, de la propriété des colloïdes " d'attirer » en quelque sorte et de retenir certaines substances sans qu'il soit possible d'expliquer cette fixation par l'affinité moléculaire, on est bien obligé d'attribuer aux micelles une propriété nouvelle, une forme d'énergie, qui n'existe pas dans les molécules et qu'on peut appeler affinité colloüdale ou micellaire.

L'affinité micellaire est beaucoup plus élective que l'affinité moléculaire, tous les éléments ne peuvent pas se grouper de façon à former des unités colloïdales. De même, le pouvoir d'une micelle de fixer 
un élément ou un composé étranger s'exerce de préférence sur certaines substances et pour les substances choisies, dans des limites très larges.

Ainsi, la soie peut être teinte par toutes sortes de couleurs, mais la solidité des teintures sera très différente: par exemple, l'acide picrique fixé, sera facilement enlevé par des lavages, tandis que le vert de malachite tiendra malgré tous les lavages.

Suivant les expériences de Devaux, rapportées par J. Duclaux, dans son intéressant ouvrage sur les colloïdes, le calcium est fixé par les parois cellulaires végétales, infiniment plus, quand il est sous forme de carbonate que sous forme de chlorure ou de nitrate. Le fer est fixé par les parois des tissus dans les dilutions de 1 p. 100 millions et un métal fixé peut être déplacé par un autre sans qu'il soit possible d'attribuer ces réactions à l'affinité moléculaire.

Ainsi, le lithium peut être déplacé par le cuivre, le calcium, le baryum, le fer et tous ces métaux peuvent se déplacer réciproquement dans le même colloïde.

Seule, cette affinité colloïdale élective peut expliquer les perturbations provoquées par certaines substances antiseptiques, des toxines, venins des alcaloïdes, etc., à doses extrêmement petites, dans les organismes vivants.

Au point de vue physique, les micelles diffèrent des molécules d'abord par leurs formes et leurs structures aussi peu stables et peu définies, que par la nature de leur composition chimique.

On ne connaît pas encore de méthode qui permette la détermination exacte du poids moléculaire d'un colloïde parce que ce poids, comme on devait s'y attendre, peut varier, pour le même colloïde, dans des limites considérables. 
On détermine le poids moléculaire des cristalloïdes par la cryoscopie qui consiste à mesurer au thermomètre le point de congélation d'un liquide, dans lequel on a fait dissoudre un poids donné d'une substance.

On a constaté, en effet, que le point de congélation d'une solution aqueuse d'un cristallö̈de quelconque est toujours inférieur au point de congélation de l'eau pure, et que l'abaissement de ce point de $1^{\circ}, 85$ correspond exactement au poids moléculaire exprimé en grammes de la substance dissoute. Ainsi, connaissant les poids atomiques du carbone [12], de l'hydrogène [1], de l'oxygène [16], et la formule de l'alcool $\left[\mathrm{C}^{2} \mathrm{H}^{6} \mathrm{O}\right]$, on trouve le poids moléculaire de l'alcool égal à 46 , et en ajoutant 46 grammes d'alcool à 1 litre d'eau pure, on trouve le point de congélation de cette solution de $1^{\circ}, 85$ au-dessous du point de congélation de l'eau pure.

Pour le poids moléculaire de l'amidon, évalué de cette façon: Sabanejew a trouvé plus de 30.000 ; Brown et Morris, de 20.000 à 30.000 .

Pour le glycogène: Sabanejew, environ 1.600 ; Gruzewska, plus de 200.000 .

Pour la silice dialysée $\left(\mathrm{SiO}^{2}=60\right)$ : Sabanejew, plus de 50.000 .

Pour l'albumine: Sabanejew et Alexandrow, 13.000 à 15.000; Bugarsky et Liebermann, 6. 400 .

Il est à peu près certain, que ces différences ont pour cause non pas tant les inexactitudes possibles des méthodes, que les différences réelles dans les quantités de molécules qui composent la partie inactive des micelles de mêmes produits, étudiées d'après des échantillons de provenances différentes.

On constate donc, que les différences de complexité 
entre une micelle et une molécule sont de même nature, sinon tout à fait du même ordre de grandeur que celles qui distinguent ces dernières des atomes composés, ceux-ci des atomes simples et ainsi de suite.

Les propriétés chimico-physiques de la micelle naturelle qui en font une unité de matière-énergie distincte, supérieure à celle de la molécule, consistent donc avant tout, dans le nombre, l'arrangement et le choix des molécules qui la composent, en second lieu dans ce fait, qu'une micelle contient toujours, dans son milieu intérieur une quantité notable d'eau qui, ainsi que nous le verrons plus loin, joue le rôle de catalyseur dans les réactions que la micelle subit dans son milieu intérieur et enfin dans ce fait que, grâce à sa formation de deux parties: l'une active et l'autre inactive, elle doit être animée d'un mouvement d'ensemble différent, par sa nature, du mouvement d'une molécule.

Nous verrons plus loin, en parlant de la nature de la forme d'énergie que nous avons appelée "affinité moléculaire ", quelle peut être la nature de l'affinité micellaire et en quoi elle diffère de l'autre.

\section{0. - Micelles albuminoïdes. Multiplication. Accroissement infini. Différenciation.}

La réunion d'un certain nombre de micelles simples en unités matière-énergie d'un ordre plus élevé a conduit à la formation des micelles albuminoïdes douées d'une forme d'énergie nouvelle que l'on a appelée vie ou énergie vitale, qui détermine la continuité et l'accroissement infini d'unités matière-énergie pourvues d'un certain équilibre physico- 
chimique instable, et conduit, par là, à la réunion des micelles albuminoïdes en cellules et des cellules, par différenciation, en tissus, et enfin à l'unité matière-énergie la plus complexe: la substance cérébrale qui se manifeste sous la forme d'énergie la plus complexe, elle aussi, la perisée.

\section{1. - Substance cérébrale. Pensée.}

De même que c'est l'apparition de l'électricité par la formation des électrons, que c'est l'apparition de l'affinité chimique par la formation des molécules simples, que ce sont en un mot, les formes d'énergies issues des unités matérielles à chaque étape de synthèse, qui déterminèrent par leurs caractères propres, les formations, les propriétés et les réactions des unités matière-énergie des ordres supérieurs, de même les formes d'énergie qui émanent des unités de l'ordre le plus élevé, confèrent un caractère particulier aux propriétés et aux réactions des unités matière-énergie de tous les ordres inférieurs réunies dans l'unité la plus élevée, et réagissent aussi, dans une certaine mesure, sur la somme de toutes les unités matière-énergie qui forment son milieu extérieur.

C'est ainsi que la pensée, issue de la formation de la substance cérébrale, forme d'énergie actuellement la plus complexe et la plus élevée, détermine et déterminera dans l'avenir, d'une part, l'évolution de la substañce cérébrale par de nouvelles différenciations et synthèses et par là la création de formes d'énergie nouvelles, dont il nous est impossible de prévoir la puissance et réagira, d'autre part, sur son milieu intérieur (l'ensemble du corps humain et sur son 
milieu extérieur, pour l'organiser à sa convenance, ce qui veut dire, en dernière analyse, pour obtenir des rendements parfaits.

Avant d'aller plus loin, il n'est peut-être pas inutile de représenter le simple exposé que nous venons de faire par un tableau.

\section{2. - Tableau}

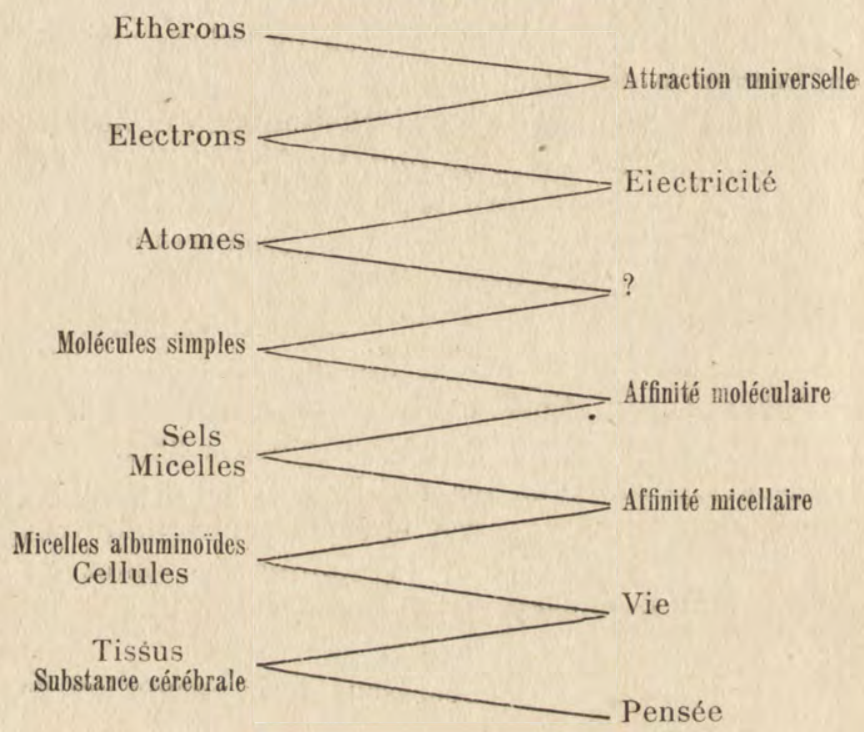

13. - Lois de l'évolution des unités matière-énergie

Dans la suite des synthèses et des différenciations dont nous venons d'indiquer les grandes étapes, il faut rappeler tout d'abord, que c'est la forme d'énergie créée par la réunion des unités d'un ordre en

\section{http://rcin.org.pl}


unités d'un ordre supérieur qui est le facteur nécessaire de cette nouvelle synthèse. C'est l'électricité qui réunit les électrons en atomes et c'est l'affinité chimique qui opère les combinaisons entre molécules simples; on peut donc admettre avec certitude que l'électricité ne peut exister autrement que dans un milieu composé d'électrons et d'éther, qu'en un mot, la forme d'énergie créée par une étape de synthèse est une énergie entièrement nouvelle, qui n'existe sous aucune forme, à aucun degré dans les unités matière-énergie d'ordres inférieurs.

Il faut en déduire tout d'abord que l'unité matièreénergie d'un ordre n'est pas la somme des unités de tous les ordres inférieurs, mais qu'elle est la résultante de la succession nécessaire des synthèses qui l'ont précédées; et ensuite, que l'évolution de la matière en unités-énergie de plus en plus complexes doit se faire pas à pas, et qu'il est impossible d'omettre un échelon quelconque dans la suite des degrés de synthèse que nous venons d'indiquer.

Il n'est pas possible d'imaginer l'existence des sels avant la formation des molécules qui ont fait naître l'affinité chimique, de même que l'existence et la vie de l'homme serait impossible dans un milieu exclusivement minéral.

\section{4. - Le connu et l'inconnu}

Mais, si nous sommes autorisés déjà aujourd'hui à formuler cette loi générale de l'évolution de la matière par des échelonș de synthèse obligatoirement successifs dans un ordre déterminé et immuable, dans lequel rien d'arbitraire ou d'imprévu ne peut arriver, parce que nous constatons que, dans notre 
monde, les choses se sont passées réellement ainsi, et qu'il nous est impossible d'imaginer qu'elles puissent se passer autrement, nous sommes encore bien loin de connaître le mécanisme intime de toutes ces formations successives.

Il y a encore bien plus d'inconnu que de connu dans le problème qu'il s'agit de résoudre, et nous serons obligé de nous borner ici, dans la plupart des cas, à réunir les éléments de ce problème et à le poser d'une façon compréhensible en rapport avec le niveau de nos connaissances actuelles.

Ainsi, en faisant le bilan de ce que nous savons et de ce que nous ignorons encore, nous pouvons affirmer l'existence réelle de toutes les unités " matière " depuis l'électron jusqu'à l'homme (ou le cerveau) et le fait qu'elles sont nées les unes à la suite des autres par une série de synthèses successives pour la matière inorganique, synthèses et différenciations pour la matière organisée, mais nous n'avons que des notions un peu précises sur la structure intime de ces unités, que pour les atomes et un certain nombre de molécules composées (cristalloïdes). Nous ne connaissons pas la structure intime des micelles, mais nous avons quelques notions sur la composition élémentaire ainsi que sur les détails de structure visibles des cellules et des tissus.

En fait de formes d'énergie, nous connaissons les lois de l'attraction universelle, qui semble être la propriété des éthérons, celle de l'électricité et de l'affinité moléculaire. Nous ignorons complètement la forme d'énergie qui réunit les électrons ou les atomes simples en atomes composés et nọs n'avons que quelques notions générales sur l'affinité mice!laire, sur la vie et sur la pensée. Nous en connaic.- 
sons les effets. Nous pouvons nous faire quelques idées sur le "comment" de leur action, mais nous en ignorons complètement le mécanisme intime, parce que ce mécanisme est fonction de leur structure intime, que nous ignorons complètement.

Toutefois, en nous résignant pour le moment à ne suivre et à ne voir que les plus grandes routes, les règles les plus générales de l'évolution, sans nous arrêter aux détails, qui nous conduiraient à des impasses impossibles à franchir et rendraient tout cet exposé incompréhensible, nous trouverons dans l'étude des conditions de milieux, des variations de ces milieux, des rapports entre ces milieux et les unités distinctes qui se sont formées dans leur sein et vivent à leurs dépens, un ensemble de notions suffisamment précises pour comprendre le mécanisme extérieur de cette synthèse spontanée dans le passé et pour formuler quelques règles de l'évolution de l'avenir.

\section{5. - Constitution de l'univers. Formation des unités cosmiques}

Les observations spectroscopiques des astres qui nous permettent d'étudier et d'apprécier aujourd'hui non seulement la composition chimique des soleils, mais aussi leurs températures ainsi que leurs mouvements de déplacement dans tous les sens, même dans le sens du rayon visuel, nous apprennent que le grand univers infini est formé de régions stellaires que l'on pourrait appeler "unités cosmiques".

L'unité dont notre soleil fait partie a, dans son ensemble, la forme d'un disque ou d'une lentille très aplatie, dont la grande circonférence est délimitée 
par la voie lactée et dont notre soleil occupe un point plus voisin du centre que de la périphérie.

A cet univers limité par la voie lactée, on a donné le nom de galaxie et on a trouvé que notre galaxie est composée d'environ deux milliards de soleils visibles, analogues au nôtre et dont les plus brillants ont une température de surface voisine de $15.000^{\circ}$. Tous ces soleils décrivent des orbites autour d'un centre idéal en se déplaçant avec une vitesse moyenne de 25 kilomètres par seconde et en formant deux 'courants de sens opposé.

Dans la direction perpendiculaire au grand axe de notre galaxie on trouve des nébuleuses dont le nombre, aujourd'hui inconnu, est d'environ un million et on a tout lieu de supposer que ces nébuleuses sont autant de galaxies analogues à la nôtre.

Les distances entre les galaxies, par rapport aux distances qui séparent les étoiles de notre galaxie, sont proportionnelles à leurs dimensions respectives. Elles ne peuvent être évaluées que par des milliers de siècles de lumière. (La lumière met 10 minutes pour arriver du soleil à la terre à raison de 300.000 kilomètres par seconde.)

Y a-t-il encore des ensembles formés par des millions ou des milliards de galaxie réunis en unités distinctes? Ces unités, les plus vastes que nous puissions admettre actuellement d'après les données astronomiques connues, de même que les galaxies et les systèmes solaires ont-ils des énergies propres ? Des énergies du milieu intérieur et des énergies rayonnantes, comme en possèdent toutes les autres unités matière-énergie que nous connaissons ?

L'avenir nous le dira! Pour le moment nous n'avons aucun moyen de l'apprécier. 
Nous pouvons done bien admettre qu'il y a dans l'espace des régions dans lesquelles la matière se trouve actuellement à un quelconque de tous les degrés d'évolution ascendante que nous venons d'énumérer, que, par conséquent, il pourrait exister une ou des régions dans lesquelles l'espace serait rempli exclusivement d'éther. Dans un tel espace, le milieu serait donc exclusivement l'éther, les unités matièreénergie les éthérons, les conditions de ce milieu : une température de zéro absolu. Si un tel milieu était complètement isolé de toute autre région de l'espace, il resterait probablement toujours dans un état d'inertie complète. Mais une telle supposition est impossible; nous savons, en effet, que d'autres régions de l'espace sont peuplées de mondes qui se trouvent au même moment à tous les autres degrés d'évolution que nous connaissons et qui émettent toutes sortes de formes d'énergie rayonnantes qui se propagent à travers tous les espaces qu'elles qu'en soient les dimensions (rayons $\mathbf{X}, \gamma$, ultra-violets, lumineux, caloriques, électriques, etc.). En outre, tout espace, quels qu'en soient les dimensions ainsi que l'éloignement des mondes constitués, doit être traversé par des particules matérielles de l'ordre de grandeur des électrons, des atomes ou même des molécules simples ou composées. On ne peut donc pas admettre un milieu complètement inerte, parce que les unités de tout éther d'un espace quelconque doivent être nécessairement animées de vibrations transmises par les énergies rayonnantes des mondes qui l'entourent.

Les particules matérielles qui entrent dans ce milieu en mouvement deviennent par leur masse relativement énorme, des centres de condensation 
des éthérons animés déjà de mouvements propres et cette condensation a pour effet une élévation progressive de température qui, à la suite, et concurremment avec l'attraction universelle, devient la condition de milieu, le facteur le plus important des formations suivantes et notamment des atomes et des molécules, et par là, d'un nouveau facteur de l'évolution ascendante : l'affinité moléculaire ou chimique.

L'affinité chimique est la forme d'énergie dont nous connaissons le mieux les lois et les effets; aussi, avant d'aller plus loin, est-il nécessaire de bien la définir, parce que cela nous aidera beaucoup à comprendre le rôle de certains autres facteurs dans l'évolution et notamment de la structure et de la sélection.

\section{6. - Influence de la température et de la pression sur les manifestations de l'affinité chimique}

L'eau est un composé relativement très stable. On peut la refroidir ou la chauffer, elle deviendra de la glace ou de la vapeur, la combinaison de deux molécules d'hydrogène avec une molécule d'oxygène n'en sera pas changée. Il faut la chauffer à plus de $1000^{\circ}$ pour que les deux éléments redeviennent libres, mais alors ils resteront indifférents l'un pour l'autre, non seulement à ces hautes températures, mais aussi à toutes les températures inférieures à $500^{\circ}$. L'affinité entre ces deux éléments ne se' manifeste sous forme d'attraction qu'à la température d'environ $500^{\circ}$, elle devient de la répulsion aux environs de $1000^{\circ}$.

Toutefois, ces deux limites seront déplacées quand on fera varier la pression qui agit en sens contraire 
de la température. Sous des pressions très élevées, l'eau restera de l'eau et l'hydrogène se combinera avec l'oxygène à des températures beaucoup plus hautes. Or, quels peuvent être les effets sur les molécules de l'action des variations de la température et de la pression?

On sait que la température est l'effet d'un certain mouvement des électrons et qu'il y a un rapport constant entre l'ampleur et la vitesse de ces mouvements et les degrés de la température. Plus la température est haute, plus grandes sont l'ampleur et la vitesse des mouvements des électrons et vice versa. En règle générale, la chaleur augmente le volume des corps, le froid le diminue et ce phénomène ne peut être expliqué autrement que par une dilatation ou un rétrécissement des atomes et des molécules ou, en d'autres termes, par l'allongement ou le racourcissement des diamètres des orbites que les électrons négatifs décrivent autour de leurs noyaux.

La pression agit en sens inverse. En augmentant ou en diminuant, elle a pour effet de diminuer ou d'augmenter les volumes des molécules, ainsi que les vitesses des mouvements des électrons.

On peut donc dire que, pour chaque couple d'éléments qui peuvent se combiner, les états de leur inertie réciproque, de leur combinaison ou de leur répulsion sont déterminés par les volumes des molécules et les vitesses des mouvements de leurs électrons; qu'en un mot, pour chaque couple d'éléments, il y a des constantes de volume et de vitesse dans lesquelles ces trois effets peuvent être réalisés et que ces constantes d'états intérieurs des molécules correspondent à certaines constantes du milieu extérieur et notamment de température et de pression.

Danysz - La genèse de l'énergie psychique. 
Or, les constantes de combinaison sunt très différentes pour les différents couples d'éléments qui peuvent se combiner: le sodium peut se combiner avec le chlore au-dessous de zéro, l'hydrogène ne se combine avec l'oxygène qu'à $500^{\circ}$, le fer avec l'oxygène à plus de $1000^{\circ}$, et les causes de ces différences ne peuvent être cherchées que dans les différences de structure intime des molécules. On peut donc dire que la structure des molécules, en corrélation avec les conditions de milieu, détermine un choix des éléments, plus généralement des unités matièreénergie, qui doivent participer à la formation des unités d'un ordre plus élevé dans les conditions de milieu de chaque moment.

\section{7. - Sélection par la décroissance progressive de la température et de la pression}

Ainsi, à ne considérer pour le moment que la période de l'évolution de la matière-énergie à partir de l'éther jusqu'aux molécules simples, nous pouvons nous représenter la marche des choses de la façon suivánte :

La condensation des unités de l'éther dans une région de l'espace a eu pour conséquence, d'une part, une élévation de température; d'autre part, une augmentation de la pression. Ces deux formes dénergie agissant en sens contraire, elles ont dû se limiter réciproquement, de sorte que l'accroissement de la température et de la pression a dû s'arrêter à un moment donné et ne pas dépasser un certain maximum.

A partir de ce maximum, qu'il nous est impossible d'évaluer, parce qu'il devait varier beaucoup suivant 
les dimensions de l'espace occupé et la quantité de matière intéressée, la dispersion dans l'espace environnant de la chaleur et d'autres énergies rayonnantes, ainsi que de certaines particules matérielles, les plus légères, a eu pour effet une diminution graduelle de la température et de la pression, et c'est ainsi que ces conditions de milieu sont devenues graduellement favorables à une série de sélections et de synthèses successives.

Toutefois, en admettant que les molécules se sont formées aux dépens des atomes, ces derniers aux dépens des électrons et enfin les électrons aux dépens de l'éther, nous constatons que toutes les unités de l'éther n'ont pas participé à la formation des électrons, ni tous les électrons à celle des atomes et des molécules, parce que dans un monde comme le nôtre, à côté de toute une série d'unités synthétisées successivement les unes par les autres, nous trouvons en même temps de l'éther, des électrons, des atomes, etc., à l'état libre (1).

Il est donc intéressant de noter qu'il y a eu une sélection à chaque étape de synthèse, et puisqu'il y avait cette sélection déjà à partir de l'éther, il faut nécessairement en déduire qu'il y avait aussi des

(1) L'existence simultanée des unités de matière de tous les ordres peut etre expliquée aussi par ce fait que les différentes régions du même espace pouvaient se trouver dans des conditions de milieu différentes, ou encore que ce fait est dû à une certaine loi d'équilibre qui régit les combinaisons et qui veut que les éléments contenus dans un espace ne se combinent jamais tous jusqu'au dernier: les atomes de chlore et d'hydrogène contenus dans un espace limité ne se combinent jamais tous pour former de l'acide chlorhydrique, il en restera toujours un certain nombre à l'état d'atome libre. Ces faits n'ont pu avoir qu'une influence de second ordre sur la loi de sélection déterminée par les modifications générales du milieu. 
différences de structure déjà parmi les unités de l'éther, parce que seules les différences de structure, et par là de stabilité et de durée, peuvent nous expliquer pourquoi, dans les mêmes conditions de milieu, certaines unités sont choisies pour participer à la synthèse d'un ordre plus élevé, tandis que d'autres restent libres et inertes.

Mais, quoi qu'il en soit de ces différences de structure des éthérons et des électrons qu'il nous est impossible d'apprécier autrement aujourd'hui, nous constatons avec certitude qu'il y a une sélection à chaque étape de synthèse et, ce qui nous importe de faire ressortir dès à présent, c'est que, dans l'évolution de la matière et des énergies, il faut considérer, d'une part, la formation d'unités matièreénergie de plus en plus complexes, dont les propriétés générales résultent d'une sélection opérée parmi les unités de l'ordre précédent; d'autre part, la formation d'un milieu extérieur, lui aussi de plus en plus complexe, formé, pour les unités de synthèse les plus élevées à un moment donné, par la somme de toutes les unités de tous les ordres inférieurs restées libres.

Ainsi pour l'homme, ou plutôt pour le cerveau, le milieu extérieur est formé tout d'abord par le reste de l'organisme humain et ensuite par la somme des unités du reste du monde animal, végétal, organique et inorganique, c'est-à-dire molécules, atomes, électrons, et éthérons libres.

Et il y a cette différence entre le milieu intérieur de l'homme et son milieu extérieur, que le premier contient en lui toutes les unités matière-énergie organisées de telle façon, que les énergies cinétiques propres à chacune de ces unités sont devenues en partie potentielles par leur association en un ensem- 
ble distinct, tandis que le milieu extérieur de l'homme contient toutes ces mêmes unités libres, c'est-à-dire non organisées dans un ensemble distinct et ayant conservé chacune pour elle son énergie cinétique propre.

On peut donc dire que l'unité de matière cerveau et son énergie cinétique, pensée, est la résultante de la sélection et de la synthèse de toutes les sélections et synthèses des unités matière-énergie qui l'ont précédée, tandis que son milieu extérieur est la simple somme des unités restées libres de toutes ces mêmes synthèses.

\section{8. - Propriétés catalysantes de l'eau}

Mais revenons à l'atome et voyons comment, par quelle suite de synthèses spontanées, par la mise en œuvre de quelles formes d'énergie nouvelles on peut arriver jusqu'à l'homme sans faire intervenir d'autres facteurs que ces mêmes énergies fournies successivement par la nature.

En perdant progressivement de la chaleur, la surface de la terre est arrivée au degré de température inférieure à $100^{\circ}$ permettant le dépôt de l'eau à l'état liquide.

C'est là le moment le plus important pour l'évolution progressive des unités de tous les degrés de synthèse qui vont suivre, parce que toutes auront désormais l'eau comme unique, indispensable milieu de toute réaction de synthèse spontanée au delà des sels.

S'il n'y avait pas d'eau à la surface de la terre, toutes les substances qui seraient déjà à l'état solide à une température inférieure à $100^{\circ}$, et ce serait 
l'immense majorité des corps qui forment la croûte terrestre, resteraient dans un état d'inertie qui, avec l'abaissement progressif de la température ne pourrait que devenir de plus en plus complète parce que, dans un milieu dépourvu d'eau, les réactions entre les éléments de ces substances exigent des températures beaucoup plus élevées.

Dans l'eau, toutes les réactions deviennent possibles à des températures comprises entre 0 et $100^{\circ}$, parce que l'eau a la propriété de dissoudre tous les sels et de les dissocier en éléments simples et en ions et, ce faisant, d'amener les molécules de tous ces éléments à leurs constantes de réaction. On peut dire que l'eau ou le milieu eau, réalise pour presque tous les éléments des conditions de réaction uniformes et remplace l'action de la température et de la pression qui, pour produire les mêmes effets sans intervention de l'eau, devraient être beaucoup plus élevées et très différentes pour les différents couples d'éléments; qu'en un mot, l'eau fait "revivre" un très grand nombre d'éléments et de leurs composés et rend bien plus uniformes les conditions de leurs réactions réciproques.

Quel peut être le mécanisme de cette action de l'eau?

Nous avons vu plus haut, en parlant de l'affinité chimique, que les états d'inertie, de combinaison ou d'attraction et de répulsion réciproques des éléments qui peuvent se combiner entre eux, sont déterminés par les volumes de leurs molécules et les vitesses des mouvements de leurs électrons, qu'il y a donc pour chaque couple de molécules un état "volume-vitesse " dans lequel les deux éléments restent inertes, un autre dans lequel ils s'attirent et se combinent 
et un autre encore dans lequel ils se repoussent; et enfin, nous avons vu aussi, que ces états sont fonction de l'action combinée de la température et de la pression, différentes pour les différents couples d'éléments.

Or, nous venons de voir que l'eau peut réaliser les conditions de combinaison et de répulsion pour tous les éléments à une température presque uniforme et nouś sommes en droit d'en conclure :

$1^{\circ}$ qu'en provoquant des réactions de dissociation et de combinaison, l'eau amène les molécules aux constantes "volume-vitesse " nécessaires à la réalisation de ces réactions;

$2^{\circ}$ qu'elle ne peut réaliser ce travail qu'en agissant par la vitesse des mouvements de ses propres molécules sur les volumes-vitesses des éléments qu'elle tient en solution.

Dans certains cas, l'eau agit par sa seule présence en dissociant les molécules composées et en rendant ainsi aux molécules simples et aux atomes les énergies cinétiques qu'ils ont momentanément perdues en se solidifiant; dans d'autres cas, l'eau participe aux réactions en dissociant ses propres molécules, mais dans l'un comme dans l'autre cas, il y a là une action que, depuis Berzélius, on appelle action de catalyse.

L'eau est le catalyseur le plus général et le plus actif pour toutes les substances qui existent à l'état solide à une température comprise entre 0 et $100^{\circ}$, elle ramène toues les réactions spontanées de la nature à une température très peu variable, sinon absolument constante. 


\section{9. - Sélection par l'eau}

Mais si l'eau dissout et dissocie toutes les substances plus ou moins simples ou complexes qui forment aujourd'hui la croûte solide de la terre, ainsi que les gaz qui forment l'atmosphère, elle ne le fait pas pour toutes dans les mêmes proportions. Il y a des éléments et des composés presque insolubles et d'autres qui sont déliquescents, et cette différence de solubilité devient le facteur le plus important des synthèses plus élevées, parce qu'il détermine la sélection et les proportions des éléments qui en feront partie.

Il est évident, en effet, que, dans la période des températures élevées, l'abaissement progressif de la température éliminait des réactions possibles tous les éléments qui se solidifiaient successivement et devenaient par là de plus en plus stables. Dans la période de l'eau, le degré de solubilité éliminera des réactions les substances les moins solubles et les plus stables, et ce sont les propriétés des composés les plus solubles et les moins stables qui détermineront les caractères et les propriétés générales dominantes des unités matière-énergie suivantes: les micelles.

Toutefois, avant d'arriver aux micelles, pour bien comprendre la nature et les réactions de ces substances, il nous faut faire ressortir encore quelques particularités des solutions aqueuses.

Certains sels d'argent sont presque insolubles dans l'eau. Il en faut 800 litres pour dissoudre, par exemple, 1 gr. d'iodure d'argent, mais on peut dissoudre 1 gr. de ce sel dans 1 c. c. d'eau, à la condition d'enlever les molécules dissoutes à mesure qu'elles apparaissent en solution. La solubilisation d'un sel n'a 
donc rien d'absolu, elle dépend d'un certain état d'équilibre, ou encore d'une réaction des molécules devenues libres sur celles, qui font encore partie du solide. On peut la modifier en neutralisant l'action des molécules dissoutes par un procédé chimique ou par un procédé purement mécanique.

En ajoutant à une émulsion de 1 gr. d'iodure d'argent dans 10 c. c. d'eau, du sulfate de potassium, par exemple, il se formera du sulfate d'argent encore moins soluble que l'iodure et qui formera un précipité, neutralisant ainsi les molécules d'iodure d'argent déjà dissoutes. Ce sera la neutralisation chimique, mais dans ce mélange il se formera en même temps de l'iodure de potassium qui neutralisera l'action des molécules d'iodure d'argent en solutions d'une façon mécanique.

En effet, en versant une solution concentrée d'iodure de potassium sur un précipité d'iodure d'argent, ce précipité se dissoudra très rapidement dans une très petite quantité d'eau, et cette action dissolvante sera purement mécanique, parce qu'il n'y a pas de réaction chimique possible entre l'iodure de potassium et l'iodure d'argent. Ce sont certainement' les mouvements des molécules de l'iodure de potassium en solution dans un milieu très concentré, c'est-à-dire très dense, qui empêchent les molécules d'iodure 'd'argent en solution de réagir sur celles qui sont encore solides et permettent ainsi la dissolution d'une grande quantité d'iodure d'argent dans une quantité d'eau relativement très petite. Il suffit, en effet, d'ajouter une certaine quantité d'eau pure à ce mélange parfaitement limpide pour reprécipiter l'iodure d'argent.

On peut donc faire entrer aussi l'action dissolvante 
du K.I. sur le $\mathbf{A g I}^{2}$ dans le cadre des actions de catalyse.

\section{0. - Milieu intra-moléculaire et intra-micellaire}

Ici, il nous faut de nouveau revenir en arrière pour parler d'un sujet que nous ne pouvions traiter jusqu'à présent sans anticiper sur le rôle de l'eau dans les réactions. Il s'agit de nous faire une idée de ce que peut être, par quoi doit être rempli l'espace compris entre les électrons qui composent un atome, entre les atomes simples dans le milieu intérieur d'un atome composé et d'une molécule composée plus ou moins complexe, enfin quel peut être, sous ce rapport, le milieu intérieur d'une micelle.

Je n'ai pu trouver aucun renseignement sur ce sujet dans les ouvrages de chimie et de physique que j'ai pu consulter, pas plus d'ailleurs que sur la question de savoir par quoi est occupé l'espace compris entre les atomes ou les molécules, qui étant continuellement en mouvement, ne peuvent pas adhérer les uns aux autres.

Logiquement, comme simple déduction de ce qui précède, on peut admettre que pour les molécules et les unités inférieures, l'espace libre du milieu intérieur doit être rempli par l'éther et les espaces intermoléculaires doivent contenir, en plus de l'éther, des électrons libres et peut-être aussi certains atomes simples libres, que nous ne connaissons pas encore; que dans le milieu intérieur des micelles, il y a des molécules libres d'eau. La quantité de l'eau intramicellaire doit varier autant que les poids et les proportions respectives des parties active et inactive des micelles; son rôle doit être celui de catalyseur 
qui délimite les réactions des micelles d'une façon encore plus étroite que celles des cristalloïdes. A de très rares exceptions près, les réactions entre les colloïdes qui composent la matière vivante, ne sont possibles qu'à des températures comprises entre $10^{\circ}$ et $55^{\circ}$. L'affinité micellaire des colloïdes naturels ne peut done s'exercer que dans ces limites de température et elle devient par là le facteur d'une sélection nouvelle d'éléments et de composés qui peuvent être actifs et réagir à cette température.

\section{1. - Formation des micelles organiques}

La structure intime des micelles nous est inconnue; toutefois, en nous représentant un atome comme un soleil entouré de planètes ou, ce qui serait peutêtre plus exact, comme une planète entourée d'un nombre plus ou moins grand de satellites, et une molécule simple comme un système solaire composé d'un soleil au centre et d'un plus ou moins grand nombre de planètes entourées de leurs satellites, nous serons obligés de nous figurer une micelle organique comme un vaste univers composé de centaines et de milliers de systèmes solaires, le tout ayant de l'eau pour milieu extérieur et également de l'eau comme milieu intérieur.

Nous ne connaissons pas de micelles albuminoïdes libres qui se seraient formées spontanément dans la nature; nous n'avons encore trouvé aucun moyen de savoir s'il s'en forme ençore spontanément aujourd'hui et nous ne savons pas en préparer dans nos laboratoires. Nous ne savons pas s'il s'en forme actuellement, parce que les milieux naturels dans lesquels il pourrait s'en former sont toujours peuplés 
d'êtres vivants, et comme nous savons que tous les êtres vivants produisent ces substances colloïdales, nous pouvons toujours supposer que celles que nous trouverions dans ces milieux naturels proviennent précisément de ces êtres vivants.

Pourtant nier la formation des micelles organiques avant la formation des cellules, ce serait nier toute l'évolution, dont nous avons aujourd'hui des preuves indiscutables; ce serait tout aussi impossible que d'admettre l'apparition des animaux avant celle des plantes, sans lesquelles les animaux ne pourraient pas vivre et qui en sont donc le milieu d'évolution indispensable.

Quelles que soient nos croyances et nos idées sur l'origine de l'univers, nous sommes absolument obligés d'admettre, que la partie de cet univers accessible à notre conscience, n'a pas été créée de toutes pièces, telle que nous la voyons aujourd'hui, qu'il y a eu, depuis l'éther jusqu'à l'homme, une évolution par une série d'étapes successives, dont aucune n'a pu être sautée, parce que lé travail indispensable à la formation des unités d'une quelconque de ces étapes n'a pu être formé que par l'énergie qui s'est créée dans les unités de l'étape précédente.

Aussi sommes-nous bien obligés d'admettre qu'il y a eu formation des micelles ou, plus généralement, des substances colloïdales organiques avant la formation des cellules vivantes, des êtres unicellulaires.

La complexité de structure et de mouvements de ces micelles, formées d'une trentaine d'espèces chimiques différentes qui peuvent former entre elles des millions (1) de composés différents peu stables,

(1) Au moins autant qu'il y a actuellement d'espèces diffé- 
ne nous permet pas encore d'en pénétrer les détails, ni même dé les imaginer.

Cela ne peut d'ailleurs pas être l'objet de cette courte étude, qui n'a d'autre but que d'indiquer les règles générales de l'évolution de la matière et de l'énergie par la formation d'une série d'unités matière-énergie successives, sans nous occuper autrement du "comment» et du "pourquoi " de ces choses que pour montrer ce que nous savons déjà et ce qui nous en reste encore à apprendre.

Avant la découverte des substances radioactives, aucun fait précis ne pouvait nous indiquer la façon dont la matière inorganique, considérée comme inerte, pouvait devenir mobile, c'est-à-dire vivante, et les vitalistes avaient beau jeu en soutenant qu'une " énergie vitale " indépendante de la matière est apparue brusquement à un moment de la genèse et a " animé " la matière qui compose les êtres vivants.

Aujourd'hui, nous ne sommes plus réduits à nous contenter d'une hypothèse de ce genre qui, sans rien expliquer, rendait encore, de par sa nature, toute recherche inutile. Nous savons avec certitude qu'il n'y a pas de matière immobile et que l'origine de la vie peut être cherchée avec une vraisemblance scientifique suffisante dans l'organisation des formes d'énergie qui l'ont précédées et dont sont douées les unités matérielles inorganiques que nous venons d'étudier.

Nous ne connaissons donc pas la structure intime des micelles organiques et nous ne savons pas comment les composés à plusieurs degrés de complexité des 20 à 30 éléments, choisis parmi les plus com-

rentes de protistes, de végétaux et d'animaux de différents tissus et organes. 
plexes et les plus avides de combinaison, que nous trouvons dans ces micelles, se sont réunis pour former ces ensembles éminemment instables, mais d'un équilibre physico-chimique relativement très constant. Mais, en tenant compte:

$1^{\circ}$ de la série de sélections successives des éléments et de leurs composés depuis l'atome jusqu'à la micelle, sélections qui ont abouti à la réunion en unités distinctes du carbone, de l'hydrogène, de l'oxygène de l'azote en quantités dominantes et d'une quinzaine d'autres éléments caractérisés par la propriété de former avec les précédents des composés complexes et relativement peu stables, facilement dissociables, tels que le chlore, l'iode, le soufre, le phosphore, l'arsenic, le silicium, le sodium, le potassium, le calcium, le fer, le manganèse, dont un, le potassium, est sensiblement radioactif;

$2^{\circ}$ de l'action catalytique de l'eau sur la réaction de tous ces éléments et de leurs composés, ainsi que de l'action catalytique réciproque de ces composés, actions qui ramènent ces éléments et leurs composés à des constantes de réaction à peu près uniformes, ou, en d'autres termes, qui permettent une série de dissociations et de combinaisons successivement alternantes dans des conditions de milieu, c'est-à-dire de densité, de pression et de température très peu variables;

$3^{\circ}$ de la somme d'énergies rayonnantes qui doit nécessairement se dégager de toutes ces réactions qui s'opèrent à l'intérieur des micelles;

$4^{\circ}$ de ce fait qu'une telle micelle n'a pu se former que dans un milieu aqueux qui tient en solution, à l'état libre, tous les éléments dont elle est formée et que, grâce aux affinités de ses composants, elle peut 
puiser dans son milieu extérieur les éléments nouveaux qui, par leurs réactions, entretiendront l'énergie de la micelle à un niveau à peu près constant; $5^{\circ}$ et enfin de toutes les énergies rayonnantes du milieu extérieur (rayons $\mathbf{X}$ et $\gamma$ ultra-violets, visibles, caloriques, électriques, sonores, etc.), qui sont autant d'excitants de l'activité de ses composants.

On peut facilement comprendre que de telles espèces d'unités-énergie ont pu se former et vivre sans autre intervention que le concours d'une part de la somme de toutes les unités-énergie qui se sont spontanément formées avant elles comme milieu extérieur et, d'autre part, l'organisation d'un certain nombre de ces unités successivement sélectionnées en un ensemble pourvu d'un équilibre dynamique propre, comme milieu intérieur.

\section{2. - Différenciation}

Pour nous expliquer la formation des cellules vivantes et des organismes multicellulaires, nous voyons entrer en jeu la différenciation, un facteur nouveau, qui, autant qu'il est possible de le savoir aujourd'hui, n'intervenait pas dans la formation des unités-énergie d'aucun des ordres inférieurs.

On sait que les différentes cellules vivantes sont formées de plusieurs espèces de matières albuminoïdes, parce que, en décomposant par l'hydrolyse les cellules ou les tissus de végétaux ou d'animaux différents, on trouve, comme produits de décomposition, des amino-acides différents et en proportions différentes. On sait aussi, par l'examen micromorphologique et microchimique, qu'une cellule quelconque est formée de plusieurs parties distinctes: 
le plasma, dans lequel on trouve plusieurs sortes de granules, le noyau,'souvent un nucléole, l'enveloppe, qui jouent dans la vie de la cellule des rôles différents et doivent, par conséquent, avoir des compositions et des structures différentes. On a appris enfin, par des analyses biologiques, que les mêmes tissus (sang, muscles, etc.) appartenant à des animaux ou végétaux d'espèces différentes, doivent être composés de complexes de structures différentes, bien qu'il ait été imposssible jusqu'à présent de déceler les causes de ces différences par l'analyse chimique.

Or, si nous pouvons considérer comme certain, qu'un composé inorganique, quelle qu'en soit la complexité, résulte toujours de la réunion d'un certain nombre d'unités d'espèces différentes, nous constatons qu'à partir de la micelle organique, les choses ne se passent pas de la même façon.

On peut bien admettre que, dans un milieu donné, il se soit formé des micelles de plusieurs espèces différentes, mais il est plus que probable que les substances albuminoïdes différentes que l'on trouve dans le noyau, le plasma et l'enveloppe d'une cellule, ne proviennent pas de la réunion en une cellule d'un certain nombre de ces micelles d'origines différentes. Nous ne savons pas comment s'est formée la cellule ou l'être unicellulaire le plus simple, pas plus que nous ne connaissons le mécanisme de la formation d'un atome ou d'une molécule simple, mais nous savons comment un organisme multicellulaire différencié se forme d'un ovule, et nous avons quelques raisons de supposer que la formation de différents organes dans une cellule, résulte de la réunion en un ensemble distinct d'un certain nombre des produits de la multiplication d'une seule 
micelle ; qu'en un mot, les différences constatées à un moment donné résultent d'une différenciation postérieure à la réunion d'unités qui étaient à l'origine identiques entre elles.

On peut donc admettre que la différenciation dans une cellule, est une conséquence obligatoire de la multiplication d'une micelle en un plus ou moins grand nombre de micelles tout d'abord identiques et qui deviennent différentes parce qu'elles restent réunies, comme la différenciation d'un organisme multicellulaire résulte de la multiplication d'un seul ovule fécondé.

La raison de la différenciation, on la trouvera, ou du moins on doit la chercher, dans les différences des conditions de milieu et par conséquent de nutrition, dans lesquelles se trouvent, à un moment donné, les micelles ou les cellules par rapport à l'ensemble de l'organisme et par rapport à son milieu extérieur.

\section{3. - Cellules. Energie vitale. Tissus}

Quelle est la forme d'énergie nouvelle qui apparaîtra par la formation des micelles organiques ou albuminoïdes ?

C'est la vie ou la faculté de se nourrir, de se reproduire, de s'accroître et de se différencier en unités plus complexes.

Les phénomènes de nutrition et de multiplication s'expliquent par l'action du concours de l'affinité chimique et de l'action des catalyseurs. Pour comprendre la synthèse des micelles en cellules et de ces dernières en tissus, il faut faire intervenir les facteurs structure, sélection et différenciation, et pour nous faire

Danysz. - La genèse de l'énergie psychique. 
une idée du mécanisme de l'intervention de ces trois facteurs, il est nécessaire de tenir compte de ce fait, que dans un milieu aussi complexe qu'est l'ensemble d'une micelle organique, il se fait des composés plus ou moins stables, dont la nature et les proportions sont déterminées par la nature et les proportions des éléments qui entrent en jeu; que les réactions entre les éléments moins stables sont plus actives qu'entre ceux qui sont plus stables et qu'enfin, il y a des composés dont la stabilité les fait éliminer à peu près complètement des réactions vitales proprement dites.

Il se fait donc là une nouvelle sélection entre tous ces composés, dont les plus stables deviennent les éléments de structure, tandis que les autres, les moins stables, continuent la vie active; mais les uns comme les autres concourront à la formation des unités plus complexes.

Mais, avant d'aller plus loin, il nous faut bien spécifier ici que, dans l'exposé qui va suivre, nous n'aurons pas à nous occuper de l'évolution cyclique des individus : protiste, plante, animal, dont l'existence est éphémère et que nous aurons à étudier dans les chapitres suivants. Ici, nous n'aurons à examiner que l'évolution continue de la matière qui, à partir de la formation des micelles organiques peut être appelée vivante, au seul point de vue de l'apparition des formes d'énergie nouvelles par la construction des unités matière-énergie plus complexes.

Nous constatons donc, que la réunion d'un certain nombre de micelles a donné naissance à des êtres unicellulaires, qui, par leurs réunions multiples ont construit des tissus, dont sont composés 
les êtres vivants multicellulaires, et il faut noter ici, que ces constructions successives ne résultent pas de la réunion d'unités d'espèces différentes en unités plus complexes, mais de la multiplication d'unités de la même espèce qui, une fois réunies, deviennent différentes, parce qu'elles se trouvent, les unes par rapport aux autres, dans des conditions de milieu et par conséquent de nutrition différentes.

Dans ses caractères les plus généraux, ce processus de construction, suivant certains plans déterminés, peut donc être comparé plutôt au phénomène de cristallisation qu'à des combinaisons entre complexes différents.

Nous ne connaissons pas les lois de l'énergie qui intervient dans ces constructions de plus en plus complexes, mais nous pouvons affirmer, qu'il n'y a pas de différences essentielles entre l'énergie qui détermine la réunion des micelles en cellules et celle qui détermine la formation des tissus et des organismes. Ce sont là des différences du même ordre que celles que l'on constate entre les molécules simples et les molécules composées de plus en plus complexes, dont la formation est déterminée par l'affinité chimique.

Une forme d'énergie entièrement nouvelle apparaît seulement avec la formation du tissu nerveux et de son expression la plus haute, la substance cérébrale, c'est la sensibilité consciente et la pensée.

\section{4. - Tissu nerveux}

Pour nous expliquer le mécanisme tout extérieur, pour ainsi dire, de la formation du tissu nerveux dans la matière vivante, il nous faut ici faire entrer 
en jeu les formes d'énergie du milieu extérieur à l'organisme, des énergies rayonnantes et rythmiques: lumière, son, odeur, saveur, etc., et probablement quelques autres encore, dont nous ne connaissons ni la nature ni les lois, mais dont l'action s'est manifestée par la formation de la sensibilité générale, du sens de l'espace, de l'orientation, etc...

\section{5. - Energies rayonnantes. Le son : sa nature, sa formation, son action sur la matière vivante}

Pour nous faire une idée aussi précise que possible de la façon dont ces énergies rayonnantes ont pu intervenir dans la formation du tissu nerveux, nous ne saurions mieux faire que d'étudier ce problème d'après un exemple précis, en choisissant pour cette étude une des mieux connues de ces énergies rayonnantes et notamment le son. Et ceci nous permettra en même temps de revenir ici, encore une fois, sur' la corrélation nécessaire entre l'apparition de certaines formes d'énergie et la formation de certaines unités de matière, - et de faire remarquer qu'il y a lieu d'établir une différence primordiale entre les énergies rayonnantes' et celles dont nous nous sommes occupés jusqu'à présent. Les premières sont engendrées ou résultent des mouvements d'ensemble de certaines unités de matière, du volume de ces unités et de la vitesse de leurs mouvements, indépendamment de leur composition, de leur structure et des mouvements intérieurs de leurs composants : les rayons lumineux, caloriques ou électriques peuvent être produits par les mouvements des mêmes électrons, les différences de manifestation de ces mouvements, sous forme d'ondes visibles, caloriques 
ou électriques ne sont dues qu'à des différences d'ampleur et de vitesse.

L'affinité chimique, l'action de catalyse, la vie, la pensée (?) qui ne rayonnent pas dans l'espace, ce sont des énergies du milieu intérieur, résultant de la structure et des mouvements intérieurs des composants des unités, qui les font naître.

On sait que le son ne se transmet pas dans le vide.

Quand on fait fonctionner un timbre électrique placé sous une cloche, dans laquelle on a fait le vide, on n'entend aucun son, on l'entend au contraire très bien quand la même cloche est remplie d'air. Cela veut dire que le son ne peut pas être transmis par un milieu privé de molécules, ou plus exactement, très pauvre en molécules, ou encore dans lequel les molécules d'air ou d'un gaz quelconque sont séparées les unes des autres par des espaces relativement très grands.

On sait aussi, que ce même espace ou milieu, appelé éther, dans lequel le son ne se produit plus ou devient imperceptible, peut encore transmettre la lumière, la chaleur, l'électricitś et une série d'autres formes d'énergies rayonnantes.

En admettant qu'il y a quelque part dans l'univers un espace dans lequel la matière n'a pas dépassé dans son évolution le stade "électron ", c'est-à-dire serait formée uniquement d'élctrons libres et d'unités encore plus petites, on doit donc admettre aussi, que, dans un tel espace la forme d'énergie " son " n'existerait pas. Elle ne peut se produire et se manifester qu'à un degré supérieur d'évolution de la matière : au stade " molécule ».

En ajoutant encore que les électrons ne perdent aucune de leurs propriétés individuelles en se réunis- 
sant en atomes et en molécules, on doit nécessairement conclure de ce qui précède, que l'unité de matière "molécule » est une formation nouvelle, et le "son" une forme d'énergie nouvellement créée par l'apparition des molécules.

Le mouvement rythmique, producteur du son, peut donc se produire dans tous les milieux composés de molécules suffisamment condensées ou agglomérées, et si on peut affirmer que ce mouvement particulier d'un certain nombre de molécules coordonnées d'une certaine façon, se produisait dans la nature avant l'apparition des êtres vivants, il est tout aussi certain que ces vibrations des molécules ne pouvaient être perçues et enregistrées sous forme de bruit ou de son qu'à partir de l'apparition des êtres vivants pourvus d'oreilles et d'un cerveau capable d'en avoir conscience, c'est-à-dire d'un tissu nerveux différencié. Ce tissu n'existe pas chez les plantes ni chez les êtres unicellulaires; il apparaît pour la première fois chez les animaux appartenant à la classe des cœlentérés et notamment chez les méduses.

Dans l'histoire de l'évolution du phénomène son, il y aurait donc à noter que le mouvement "son " n'est devenu sensation sonore qu'au moment de l'apparition des méduses, c'est-à-dire du premier appareil nerveux capable de l'enregistrer sous cette forme et que depuis ce moment, ce mouvement des molécules a été le seul facteur du développement progressif de l'appareil de l'audition jusqu'à celui de l'hom'me, où il est devenu compréhension musicale. Il a contribué ainsi, dans une certaine mesure, au développement du système nerveux central.

Il n'est pas douteux que le mouvement son, de 
même que les autres mouvements rayonnants et rythmiques perçus aujourd'hui par nos organes des sens sous forme de la lumière, de l'odeur, de la saveur, etc., et qui existaient avant l'apparition de la matière vivante, ont été les facteurs directs et principaux, sinon exclusifs de la formation et de la différenciation du tissu nerveux et par ce dernier du développement progressif des êtres vivants. Et cette conception nous permet de nous faire une idée assez claire de l'intervention du facteur "mécanique " dans l'évolution de la vie.

En résumé, le phénomène "son " est né dans la nature, sous forme d'un. certain mouvement coordonné des unités matérielles au stade "molécule " de l'évolution de la matière. Il n'existait pas au stade "électron ». On ne sait pas quelle est son action sur la matière inerte. Pour la matière vivante, le mouvement son est devenu sensation sonore et comme tel un des factèurs du développement du tissu nerveux qui, à son tour, l'a fait évoluer en compréhension de l'harmonie musicale.

\section{6. - Intervention des énergies rayonnantes dans la}

formation des sens, de la mémoire, de la conscience et de la pensée.

Toutes les autres énergies rayonnantes ont dû agir de la même manière : en frappant d'une façon continue certains éléments de la matière vivante, éminemment plastiques et mobiles, leurs mouvements rythmiques réguliers ont imprimé un certain rythme aux mouvements de ces éléments et ont provoqué ainsi la formation des structures, ou en d'autres termes, la construction d'appareils sensibles à ces 
vibrations, c'est-à-dire de récepteurs capables de vibrer à l'unisson. C'est ainsi que le son a créé l'oreille et la lumière, l'œil.

Le mécanisme de la formation des organes de l'olfaction et du goût est un peu différent. Dans la formation de l'œil et de l'oreille, les corpuscules en mouvement n'interviennent que par leur nature physique. Une corde fabriquée en un métal quelconque donnera la même gamme de son qu'une corde en boyau ou en soie et la vibration d'un même électron donnera des couleurs différentes suivant la vitesse et l'ampleur de ses mouvements.

Dans la formation des papilles gustatives et olfactives, ce sont au contraire les propriétés chimiques des molécules qui jouent le rôle le plus important. Ce sont encore des mouvements, mais dans ce cas des mouvements du milieu intérieur, non rayonnants, de certaines molécules qui produisent des sensations de saveur ou d'odeur et provoquent la formation de cellules sensitives spéciales.

Nous ignorons encore le mécanisme intime par lequel la réception de ces mouvements rythmiques par les organes des sens a contribué à la formation et au développement des centres spéciaux dans la substance cérébrale, mais nous pouvons affirmer que les choses se sont passées ainsi, parce que. nous constatons que chez les animaux privés de certaines de ces vibrations pendant une longue série de générations, il se produit l'atrophie plus ou moins complète des sens qui correspondent à ces vibrations. Les poissons et autres animaux qui vivent dans les cavernes ou dans les profondeurs de la mer, c'est-à-dire dans l'obscurité complète, deviennent aveugles, leurs yeux ainsi que les centres cérébraux de la vision s'atro- 
phient et ils perdent ainsi l'organe de réception et l'organe de conscience de la vision. Nous savons aussi par l'expérience, qu'un entraînement et un exercice convenable peuvent développer dans une large mesure la délicatesse, la finesse et l'ampleur de toutes les sensations et par là des centres de conscience correspondants, et si nous ignorons le mécanisme par lequel la formation d'un appareil récepteur arrive à former le centre de conscience matériel correspondant, si nous ne savons pas comment de la réunion de tous ces centres se forme l'organe matériel de la pensée, nous pouvons certainement admettre, que les mêmes mouvements rythmiques souvent répétés provoquent la formation de la mémoire qui détermine la conscience, et que c'est du concours de toutes ces mémoires et de ces consciences particulières, que naît la faculté de former des idées et enfin la faculté de combiner ces idées, c'est-à-dire la pensée.

\section{7. - Comment expliquer l'absence des organes des sens chez les végétaux}

On sait que les plantes ne possèdent aucun organe des sens qui, dans le règne animal, apparaissent déjà au plus bas de l'échelle, chez les coelentérés, - on en trouve même, à l'état rudimentaire, chez certains êtres unicellulaires (velvox, vorticelle), - et il n'est pas sans intérêt de rechercher ici, quelles peuvent être les causes de cette infériorité des plantes sous ce rapport.

Pour trouver ces causes, il faut noter tout d'abord que les plantes existent à la surface de la terre depuis plus longtemps ou au moins aussi longtemps que les animaux. On peut très bien admettre, en effet, que 
déjà à partir de certains protistes, il y a eu bifurcation dans l'évolution des êtres vivants. Les uns ont continué à se nourrir exclusivement de substances inorganiques et sont restés plantes, les autres ont appris à assimiler des substances organiques provenant des produits de décomposition des cellules mortes et sont devenus animaux.

$\mathrm{Au}$ point de vue de leur nutrition, les plantes diffèrent donc des animaux, par ce fait que les premières font la synthèse totale de leurs tissus à partir des composés inorganiques simples, tandis que les animaux ont désappris à faire cette synthèse totale. Pour former leurs tissus ils sont obligés de trouver dans leur milieu extérieur des matériaux d'une structure plus élevée, préparés par les végétaux et notamment des amino-acides.

On peut dire que, pour faire œuvre de constructeurs, les végétaux sont obligés de fabriquer tout d'abord des briques et des planches avec de l'argile et du bois brut, tandis que les animaux n'ont pas à s'occuper de ces travaux préparatoires, ils emploient des briques, des poutres et des planches toutes faites.

Les animaux vivent, dans une certaine mesure, en parasites, du travail des végétaux.

Dans un autre ordre d'idées, on constate que la proportion des éléments stables, de structure ou de charpente, par rapport aux éléments de vie active, du plasma, est beaucoup plus grande chez les végétaux que chez les animaux. Pour un même poids de matière, la somme totale des unités de travail est beaucoup plus grande chez les animaux que chez les végétaux.

On constate donc que, d'une part, les végétaux ont à leur disposition des moyens plus restreints que les 
animaux pour accomplir un travail plus considérable; que d'autre part, ce travail est rendu plus pénible par la lenteur plus grande des échanges et des réactions à cause de la prédominance des éléments de structure, ce qui fait que la cellule d'un végétal est plus inerte vis-à-vis de son milieu extérieur, qu'elle est bien moins sensible aux excitations extérieures et en particulier à l'action des énergies rayonnantes rythmiques, que la cellule animale.

Les éléments de la charpente relativement très stables, sont tout aussi nécessaires au développement d'un être vivant que les éléments de vie active, du plasma; mais il y a antagonisme dans les rôles que ces deux éléments jouent dans l'évolution. Il faut un certain équilibre entre eux, pour que leur concours puisse conduire à des synthèses nouvelles plus complexes.

Une chitinisation ou une ossification trop rapide marquerait un arrêt de développement prématuré chez les animaux : le développement proportionnellement démesuré du tissu ligneux, qui absorbe presque toute leur énergie vitale, a empêché les plantes d'évoluer au delà du stade qu'elles occupent actuellement et qu'elles ne dépasseront pas, tant que le rapport entre les tissus de construction et le plasma restera le même.

On peut donc dire que l'arrêt de développement des végétaux au stade "pré-nerveux " est déterminé par leur inertie trop grande à l'action des énergies rayonnantes de leur milieu extérieur, déterminée elle-même par un développement disproportionné de leur charpente cellulosique, - et que, la formation ainsi que le développement progressif du tissu nerveux chez les animaux, a été rendu possible par l'uti- 
lisation de l'énergie dont dispose le milieu intérieur de l'organisme, au travail d'une différenciation plus haute et plus fine des tissus instables, plutôt qu'au travail de construction des tissus de charpente, relativement inertes (1).

\section{8. - Evolution du tissu nerveux et des énergies nerveuses}

\section{L'évolution progressive continue des vertébrés doit être attribuée au développement progressif du cerveau. L'évolution du pallium. - On peut admettre avec certitude que les sens de la vision et de l'ouïe se sont formés chez les animaux, sous l'action de la lumière et du son, c'est-à-dire sous l'action des énergies rayonnantes qui, par la répétition des mou- vements rythmiques qui les caractérisent, ont pro- voqué la différenciation et l'organisation de certaines}

(1) Nous ne voudrions pas trop empiêter ici sur des sujets qui seront traités avec plus de fruit dans les chapitres suivants consacrés plus spécialement à la biologie, mais il n'y a pas d'inconvénient à signaler déjà, au risque de se répéter en y revenant plus loin, que, puisqu'on cherche à intervenir sciemment dans l'évolution actuelle et future de l'homme, on peut trouver des directives intéressantes dans ce qui vient d'être dit au sujet de l'évolution des plantes.

Pour que l'évolution de l'homme puisse se faire dans les meilleures conditions, il faut une certaine harmonie dans le développement des différents tissus et organes qui composent le corps humain.

Point n'est besoin d'une longue discussion pour démontrer l'importance de l'intervention de différentes qualités de la pensée dans l'exécution de tout travail, quelle qu'en soit la nature, pour comprendre que c'est par l'intelligence que nous pouvons rendre facile et agréable tout travail qui, sans cela, demanderait des efforts beaucoup plus longs et plus pénibles, qu'en un mot, le rendement du travail cérébral sera toujours infiniment supérieur au rendement du travail musculaire.

Il n'est plus nécessaire de démontrer aujourd'hui que le sur- 
cellules, devenues ainsi des récepteurs capables d'enregistrer ces vibrations rythmiques.

Ce sont là des réactions provoquées par des agents physiques sur la matière vivante. La nature chimique des sources de lumière ou du son ne peuvent jouer dans la formation de l'œil et de l'oreille qu'un rôle probablement tout à fait secondaire.

On peut en dire autant des organes du tact, dont la formation procède pourtant d'un ordre d'actions tout à fait différent. Dans la formation des cellules sensitives tactiles, on ne peut guère faire intervenir un mouvement rythmique quelconque, mais il nous faut reconnaître que, dans l'état actuel de nos connaissances, il n'est guère possible de nous faire une idée autre sur l'origine et le mécanisme de la différenciation de ces cellules, que de dire qu'elle provient de la sensibilité générale de la matière vivante.

menage physique s'oppose au développement du cerveau et que, par un entrainement et un exercice convenable on peut développer les valeurs de la conscience, de l'imagination et de l'intelligence.

L'étude des propriétés des corps radioactifs nous a appris que l'énergie calorique renfermée dans un kilogr. d'uranium est égale à celle que l'on obtient en brûlant 400 tonnes de charbon. Tout atome confient une somme d'énergie analogue, on peut donc facilement se représenter quelle bienfaisante révolution dans l'ordre social on peut espérer du jour où il nous sera possible de retirer et d'utiliser à volonté l'énergie enfermée dans les atomes. Et il n'est pas nécessaire d'insister sur le choix des moyens à employer pour arriver à ces résultats le plus rapidement possible. Il est évident en effet qu'on n'y arrivera qu'en organisant de mieux en mieux l'instruction générale par l'établissement d'instituts de recherches scientiflques, en les dotant sans compter de tous les moyens d'action dont on peut disposer à chaque moment donné, en facilitant la sélection des cerveaux les plus capables par leurs qualités d'imagination et d'intelligence, de diriger les travaux de ces instituts. 
Les organes de l'odorat et du goût ont été formés par certaines propriétés chimiques des substances, qui ont pour les êtres vivants une odeur ou une saveur et, dans ce cas, on peut admettre aussi que la différenciation a eu pour origine un mouvement particulier des molécules.

Nous savons, en effet, que les molécules chimiques diffèrent entre elles par le nombre d'atomes dont elles sont composées, et par l'arrangement de ces atomes et surtout par la rapidité, le sens et l'ampleur des mouvements de ces atomes et de l'ensemble de la molécule, et nous pouvons bien admettre que ce n'est ni la structure ni le volume, mais seul le mouvement d'un corps qui peut provoquer un mouvement ou une vibration concordante dans un autre corps, que c'est par conséquent le mouvement rythmique de certaines molécules qui correspond à certaines propriétés chimiques, qui a provoqué la différenciation des cellules olfactives et gustatives. Nous avons probablement pour chaque saveur et chaque odeur des cellules sensitives spéciales et les substances qui ne possèdent pour nous ni goût ni odeur, sont celles qui sont complètement insolubles (dans les liquides ou dans les gaz) ou bien celles pour lesquelles nous n'avons pas de cellules sensitives réceptrices.

On peut et on doit même admettre qu'il en est ainsi, que ce sont les vibrations rythmiques du milieu extérieur qui ont provoqué la formation des premières cellules sensitives, leur groupement en organes des sens et enfin la différenciation des centres nerveux qui enregistrent ces impressions.

Nous ignorons le mécanisme intime de ces formations, parce que ce mécanisme est déterminé par des réactions chimiques qu'il nous est encore impossible 
de suivre ou d'analyser dans une cellule vivante; mais en examinant le développement du système nerveux, d'une part, dans la série animale, d'autre part, pendant la vie embryonnaire des individus, on constate, que ce sont les cellules sensitives ou les organes des sens qui apparaissent les premiers, avant l'apparition des centres nerveux.

Ainsi, chez les cœlentérés adultes (fig. 1 et 2 ), on trouve des cellules sensitives et des organes munis d'otolithes que l'on peut considérer comme des oreilles rudimentaires, sans connexion avec un organe ou un tissu, auquel on pourrait attribuer le rôle d'un centre nerveux.

Chez un grand nombre de larves d'invertébrés (vers, mollusques, arthropodes) et de vertébrés, on voit un œil, une oreille ou un organe olfactif apparaître avant le cerveau.

Au plus bas de l'échelle, chez les porifères (éponges), on ne trouve en fait d'organes des sens que des cellules tactiles. Les cœlentérés (méduses, coraux, etc.) sont également dépourvus d'un système nerveux proprement dit. On trouve les premières fibres nerveuses chez les plathelminthes (fig. 3) et les premiers ganglions nerveux, que l'on peut considérer comme un cerveau chez les nématodes (fig. 4).

A partir des cœlentérés, le système nerveux central des invertébrés se compose essentiellement d'un collier de ganglions qui entoure l'œsophage. La partie dorsale de ce collier correspond au cerveau des vertébrés. Les ganglions sus-œsophagiens (fig. 5) reçoivent les filets nerveux de tous les organes des sens. Les ganglions sous-œsophagiens se continuent en une chaîne de ganglions tout le long de la face ventrale qui, par ses fonctions, sinon par sa position par 
rapport au tube digestif, représente à la fois la moelle épinière et le système nerveux sympathique des vertébrés.

L'évolution progressive du système nerveux central des invertébrés consiste dans le développement de plus en plus considérable du ganglion céphalique dorsal ainsi que de la chaîne des ganglions ventraux. Les deux systèmes, le dorsal et le ventral, se développent parallèlement et on constate que la masse totale des ganglions ventraux forme toujours un volume plus considérable que la masse des ganglions cérébraux (fig. 6).

Il n'existe pas chez les invertébrés, même les plus développés au point de vue nerveux, tels que les abeilles ou les fourmis, d'organe nerveux central qui prédominerait nettement et de beaucoup sur l'ensemble de tous les autres ganglions, comme c'est le cas chez les vertébrés supérieurs. En nous représentant le corps d'un animal comme formé d'une série de segments accolés les uns aux autres, qui se sont différenciés de plus en plus, dans le cours de l'évolution progressive, en régions céphalique, thoracique, abdominale et caudale, on constate qu'au point de vue nerveux, cette différenciation s'est arrêtée chez les invertébrés à un certain stade, qui laisse à chacun de ces segments une certaine indépendance individuelle, parce qu'il est pourvu d'un ganglion nerveux relativement très important.

On peut dire que, quel que soit le développement de l'énergie nerveuse chez les invertébrés, que l'on peut considérer, chez les abeilles et les fourmis, comme supérieure à celle de beaucoup de vertébrés, ce développement se trouve arrêté aujourd'hui au stade de "l'instinct ", c'est-à-dire de réactions ré- 

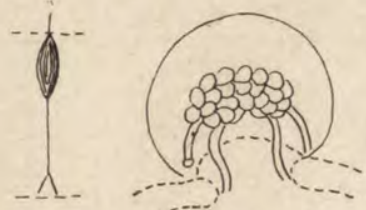

Fig. 1 .

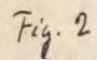

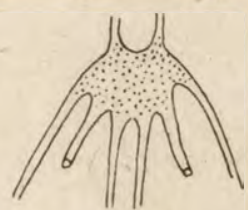

Fig. 4

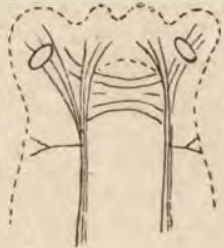

Fig. 3
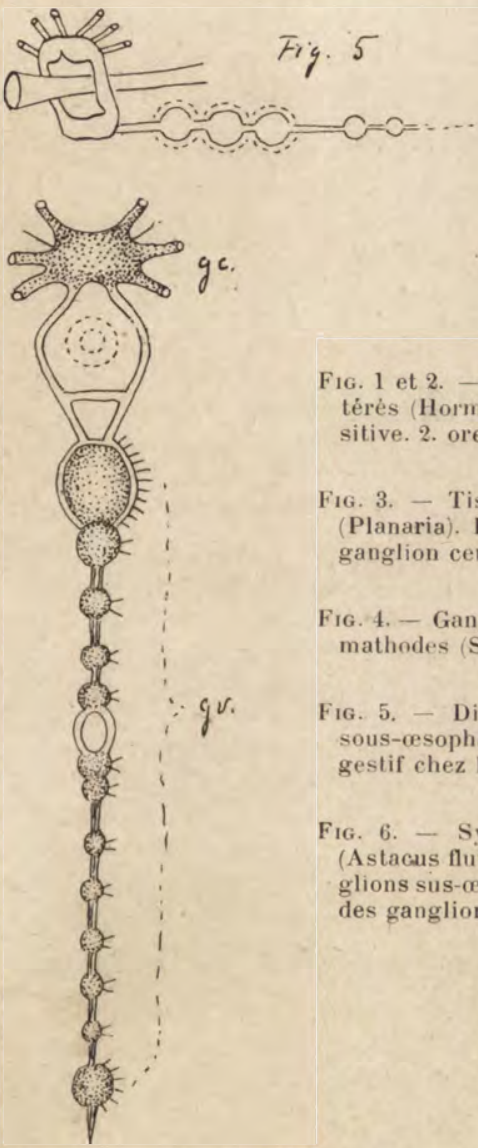

Fig. 1 et 2. - Organes des sens des Cœlentérès (Hormiphora plumosa). 1. cellule sensitive. 2 . oreille rudimentaire.

Fig. 3. - Tissu nerveux des Platelminthes (Planaria). Les yeux ne sont reliés à aucun ganglion ceniral.

Fig. 4. - Ganglions sus-œsophagiens des Némathodes (Sagitta bipemetata).

Fig. 5. - Disposition des ganglions sus et sous-esophagiens par rapport au tube digestif chez les invertébrés.

FIG. 6. - Système nerveux de lécrevisse (Astacus fluviatilis, d'après Huxley).gc. ganglions sus-œsophagiens (cerveau) gv. chaine des ganglions sous-œsophagiens. 
http://rcin.org.pl 

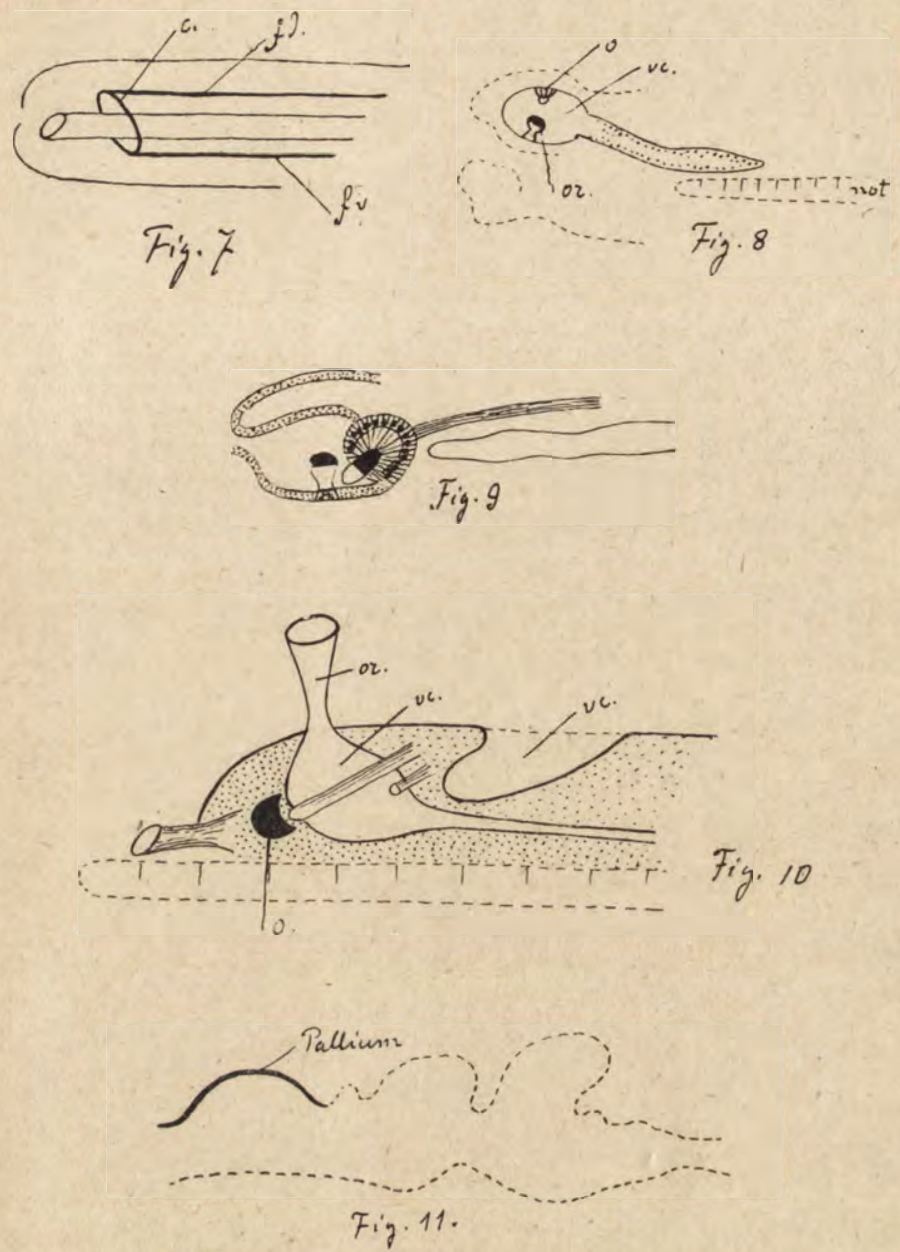

F16. 7. - Balanoglossus. c. collier nerveux. fd. filet nerveux dorsal. fv. filet nerveux ventral.

Fıg. 8. - Larve d’ascidie (d’après Seeliger), vc. vésicule cérébrale. o. ail. or. oreille. $n l$. notocorde.

FIG. 9. - Ascidia mammillata (d'après Kowalewsky).

Fıg. 10. - Amphioxus lanceolatus (d’après Hatschek), vc. vésicules cérébrales.

Fig. 11. - Encéphale de poisson. 
flexes; ce qui d'ailleurs n'exclut pas une certaine centralisation et coordination de ces réactions, dans les ganglions cérébraux; mais ce centre de coordination n'est pas assez puissant, ne contient pas de régions assez différenciées, pour permettre la formation de la pensée proprement dite, la conception d'idées abstraites.

Et l'on ne trouve, dans l'anatomie et la structure intime du système nerveux des invertébrés, aucun indice d'une tendance à un développement supérieur qui permettrait de prévoir l'élévation de l'énergie nerveuse au degré de la pensée, précisément parce que, dans les filiations que l'on peut établir, on ne voit nulle part une tendance à la formation d'un organe central de plus en plus puissant et dominant sans conteste tous les autres centres nerveux.

Il est, au contraire, assez aisé de trouver cette tendance dans la filiation des vertébrés.

On peut admettre avec Kowalewsky et la plupart des zoologistes postérieurs que ce sont les tuniciers (urochorda, ascidies) qui relient les vertébrés aux invertébrés. Ils sont reliés aux vertébrés par les acrania (amphioxus), qui ne possèdent pas encore de crâne, mais sont pourvus d'une notocorde, c'est-àdire d'une colonne vertébrale rudimentaire, aux invertébrés par les hemicordes (balanoglossus), qui n'ont pas de notocorde, mais possèdent un filet nerveux dorsal que l'on peut assimiler à la moelle épinière des vertébrés. Il manque aujourd'hui les échelons qui nous permettraient d'établir une filiation précise entre les protozoaires et les hemicordes, mais on admet généralement que les protozoaires ou les cœlentérés ont donné naissance en même temps à plusieurs types d'animaux, qui ont évolué parallèle-

Danysz. - La genèse de l'énergie psychique. 
ment et indépendamment les uns des autres : échinodermes (oursin), molluscoïves (oristatella, terebratula), vers, arthropodes, mollusques, et entre lesquels il n'y a pas de filiation à établir.

Il en est de même chez les vertébrés, ou plus exactement les chordata, dont la filiation peut être suivie depuis le balanoglossus jusqu'à l'homme, en passant par les poissons élasmobranches (chien de mer et les amphibiens et en donnant en route des branches collatérales représentées aujourd'hui par les poissons osseux, les reptiles et les oiseaux.

L'évolution progressive continue des vertébrés doit être cherchée dans le développement progressif du cerveau, qui seul peut devenir pour l'animal une source d'énergies constructives nouvelles.

J'ai cherché à rendre cette évolution plus facile à apprécier par une série de figures qui représentent d'une part les encéphales de différentes classes d'animaux (fig. 7 à 18); d'autre part, par une série de dessins schématiques (fig. 19 à 25), et je pense qu'en examinant ces dessins, il est impossible de ne pas constater les relations étroites entre la conformation anatomique et les volumes respectifs des différentes parties d'un encéphale, d'une part, et d'autre part, la puissance et la qualité de l'énergie nerveuse de l'animal étudié.

On constate ainsi qu'il y a une corrélation étroite entre le développement du cerveau antérieur, du pallium et le degré, ainsi que la qualité de l'intelligence.

On ne sait pas s'il existe chez les invertébrés des 
ganglions cérébraux, dont la structure et les fonctions pourraient être comparées au pallium des mammifères, mais on peut affirmer qu'il n'y a rien que l'on puisse comparer au pallium chez les acraniens.

On voit ce centre apparaître chez les poissons sous forme d'une mince couche de cellules nerveuses, qui devient plus volumineuse chez les batraciens, et s'accroît progressivement chez les reptiles, les oiseaux et les mammifères, pour devenir relativement énorme chez l'homme, où il recouvre complètement, par ses deux hémisphères, toutes les autres parties de l'encéphale, tandis que toutes ces autres parties, le cerveau moyen, le cervelet et la bulbe deviennent relativement plus réduites.

Nos connaissances concernant la structure intime du pallium de l'homme et des différents vertébrés présente encore de nombreuses et d'importantes lacunes et l'étude de la localisation des différentes facultés, dont elle est le siège, est à peine ébauchée.

L'expérimentation qui pourrait nous donner des notions précises sur ce sujet est difficile chez les animaux, parce qu'il est souvent impossible de trouver la relation exacte entre la lésion que l'on pratique dans les hémisphères cérébrales d'un cobaye, d'un lapin et même d'un chien et les modifications dans les manifestations psychiques qui peuvent en résulter, et qu'une telle expérimentation est tout à fait impossible chez l'homme.

Toutefois, l'étude de la structure des hémisphères cérébraux de l'homme a permis à Flechsig (1) de déduire, que l'écorce cérébrale (cerveau antérieur)

(1) Nous reproduisons ces données d'après l'ouvrage de A. VAn Gehuchten: Anatomie du système nerveux de l'homme. Louvain, 1900. 
peut être divisée, au point de vue de ses fonctions, en deux zones nettement distinctes: une zone comprenant toutes les régions de l'écorce reliées par des fibres de projection centripètes et centrifuges à des centres nerveux inférieurs: la zone des centres de projection ou les sphères sensorielles, et une zone comprenant toutes les parties de l'écorce dépourvues ou plutôt beaucoup plus pauvres en fibres de projection, mais reliées par de nombreuses fibres d'association entre elles et aux sphères sensorielles: la zone des centres d'association.

La zone des centres de projection comprend quartre sphères sensorielles : la sphère tactile, la sphère olfactive, la sphère visuelle et la sphère auditive.

La sphère tactile, la plus étendue de toutes, comprendrait les circonvolutions centrales, le lobule paracentral, la partie voisine de la circonvolution du corps calleux et la partie postérieure des trois circonvolutions frontales. Cette sphère reçoit et réfléchit toutes les impressions de sensibilité générale, perçues par les terminaisons sensitives périphériques.

La sphère olfactive comprendrait le trigone olfactif et la partie voisine de la circonvolution du corps calleux, la substance perforée antérieure, le repli uniforme, la circonvolution semi-lunaire, la circonvolution ambiante et la partie voisine de la circonvolution de l'hippocampe.

La sphère visuelle comprend la partie interne de chaque hémisphère cérébral qui entoure la fissure calcarine.

La sphère auditive embrasse la partie moyenne de la première circonvolution temporale et. la partie correspondante de cette circonvolution qui concourt à former l'opercule inférieur de la fissure de Sylvius. 


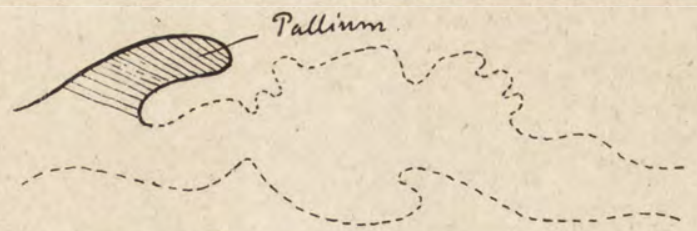

\section{Fig. 12}

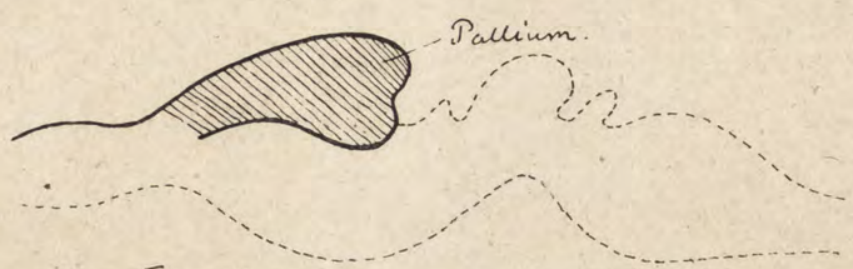

Fig. 13
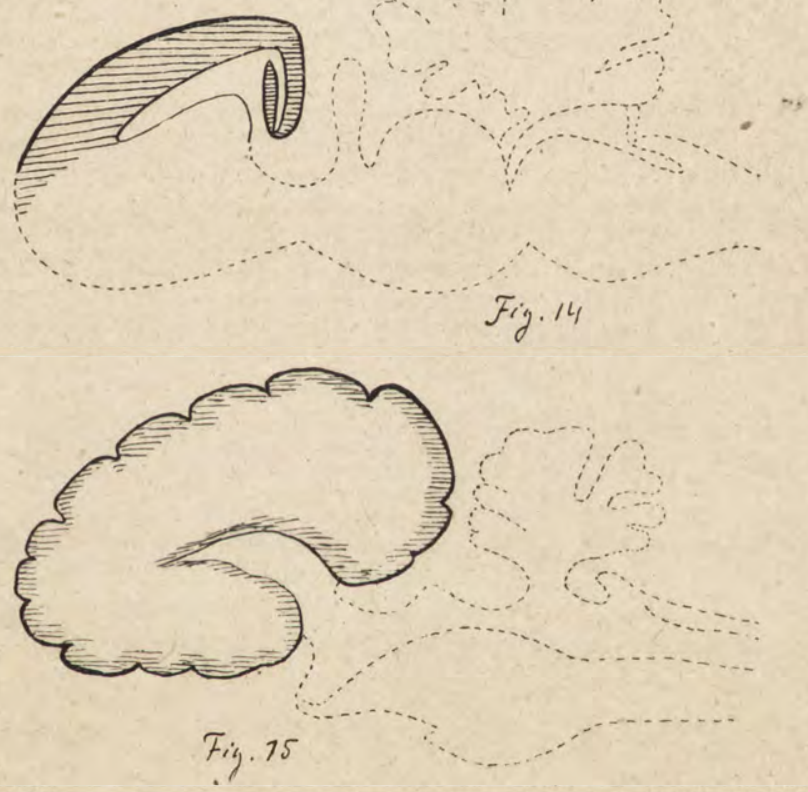

Fig. 12. - Encéphale d'amphibien.

Fig. 13. - Encéphale de reptile.

Fig. 14. -- Encéphale d'oiseau.

FiG. 15. - Encéphale de mammifère inférieur. http://rcin.org.pl 

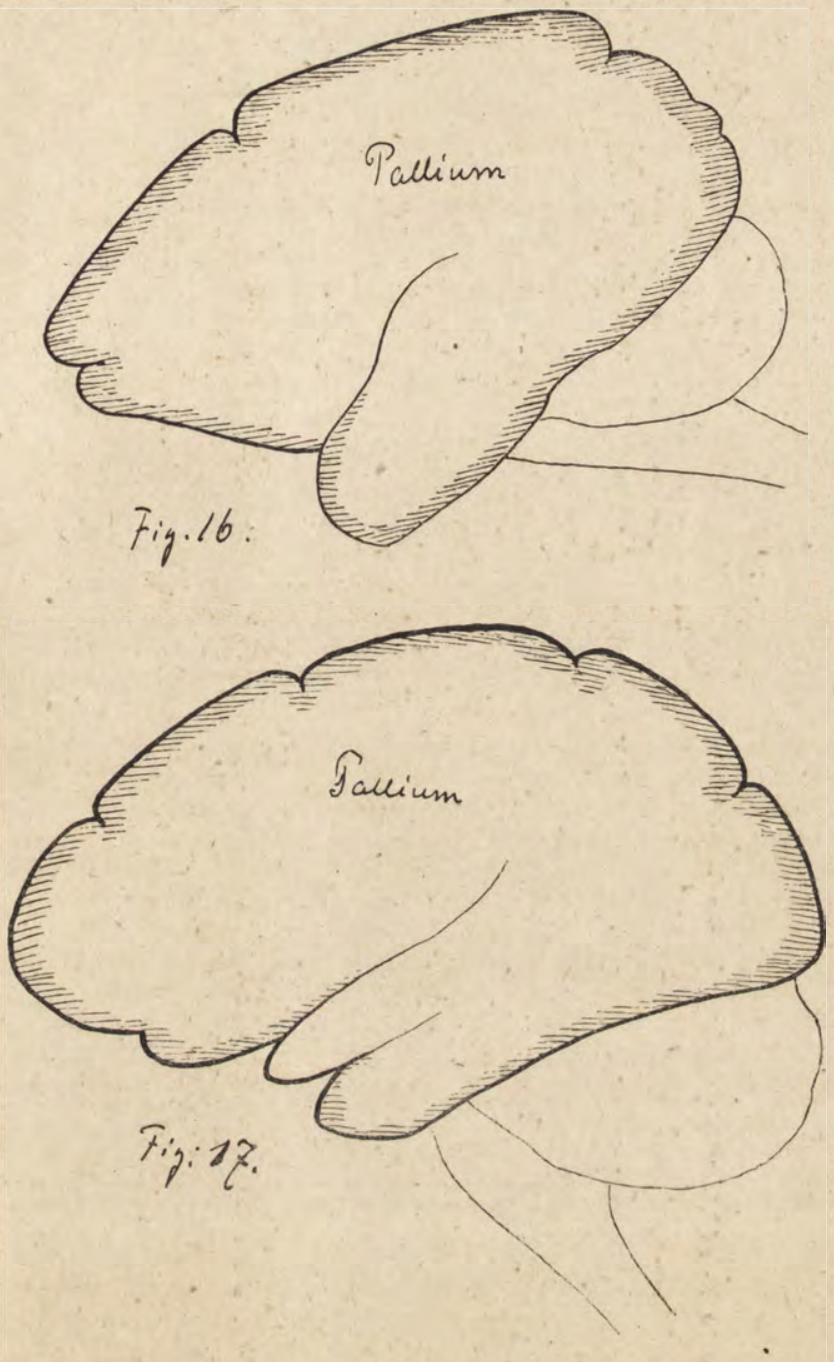

Fig. 16. - Encéphale de macaque.

Fi6. 17. Encéphale de gorille. 
http://rcin.org.pl 


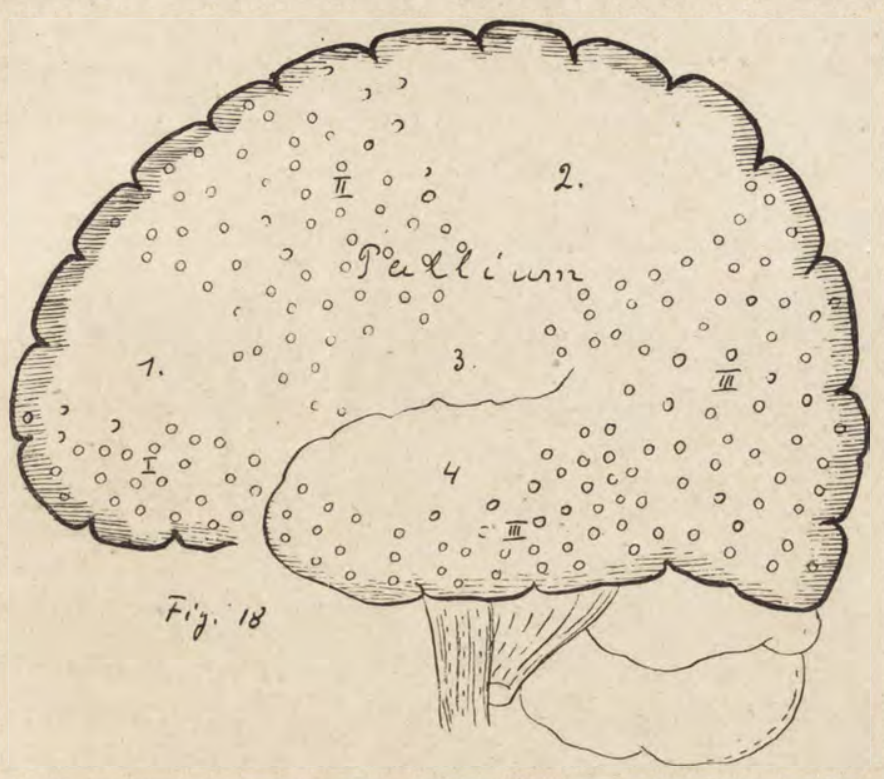

Fig. 18. - Encéphale de l'homme (Pallium).

1. Sphère visuelle. - 2. Sphère tactile.

3. Sphère auditive. -4 Sphère olfaclive.

I, II, III, Sphères d'association.

Les fig. 11 à 18 montrent le développement progressif du Pallium chez les vertébrés, depuis celui des poissons jusqu'à celui de Thomme. 
http://rcin.org.pl 

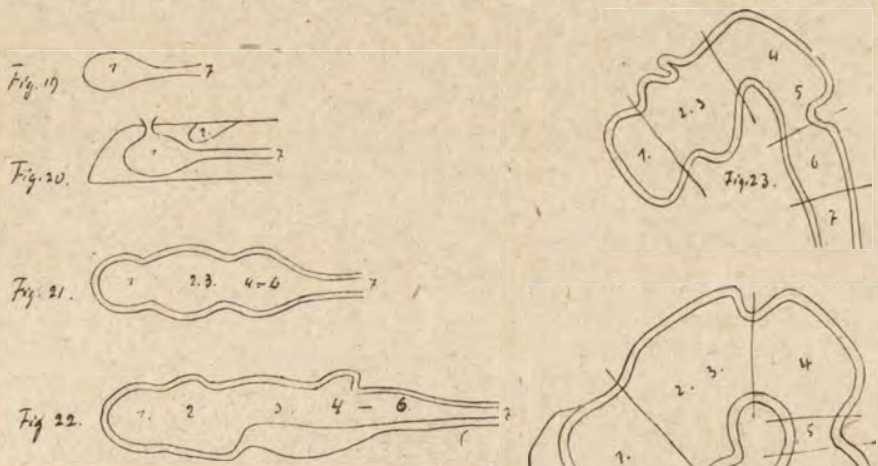

Fig. 19. - Vésicule cérébrale d'Ascidie.

Fı́. 20. - Vés. cérébr. d'Amphioxus.

Fıg. 21 et 22. - Vés. cérébr des Vertébrés inférieurs.

Fig. 23. - Encéphale dun embryon de poulet de $41 / 2$ jours.

- Fig. 21. - Encéphale đ'un embryon humain d'un mois 1 . cerveau antérieur.

2. cerveau intermédiaire. 3. cerveau moyen. 4. cervelet. 5. protubérance. 6 . bulbe. 7. mœlle épinière. L'unique vésicule de l'ascidie se subdivise successivement en six compartiments, dont les parois s'épaississent et se différencient pour devenir les régions et les centres nerveux que l'on trouve dans l'encéphale de l'homme adulte.

Fig. 25. - Encéphale d'un homme adulte.
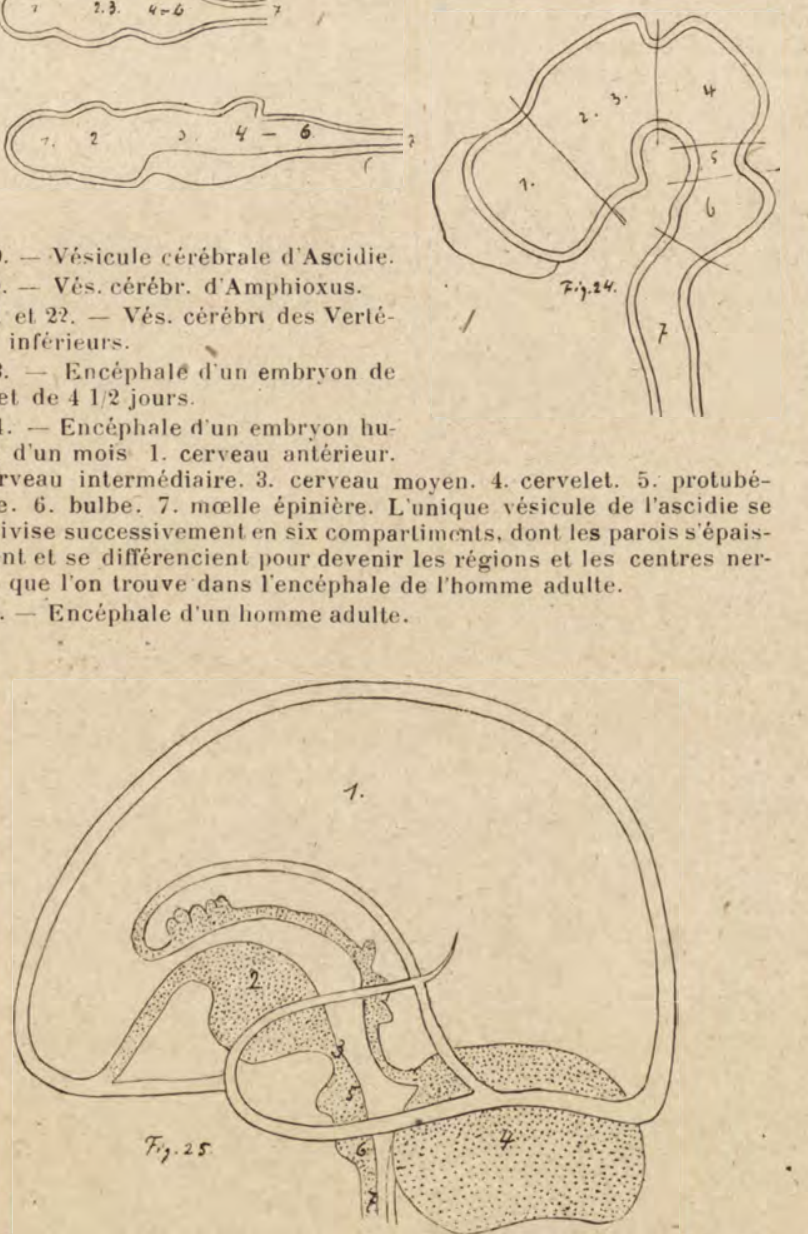

Fig. 19 à 25. - Evolulion des vésicules cérébrales depuis lascidie

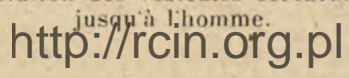


http://rcin.org.pl 
Au point de vue anatomique et fonctionnel, les sphères sensorielles représentent la projection sur l'écorce cérébrale des surfaces sensibles, dans lesquelles se terminent les nerfs périphériques; aussi leurs étendues respectives sont en rapport avec l'étendue de ces surfaces.

La sphère olfactive est la moins développée parce qu'elle n'a à desservir que la petite région de la muqueuse des fosses nasales. La sphère auditive reçoit les impressions de la surface sensible de l'organe de Corti, de l'oreille interne; la sphère visuelle, celles de la rétine. A ces trois sphères sensorielles n'aboutissent que les excitations du monde extérieur. La sphère tactile est plus étendue que les trois autres ensemble, parce qu'elle reçoit les impressions tactiles de la surface et de l'intérieur du corps tout entier et en outre celles de la sensibilité gustative, génitale, etc. Elle tient aussi dans sa dépendance tous les mouvements du corps.

La zone des centres d'association est formée de trois centres distincts : le grand centre d'association postérieur, le centre moyen et le centre antérieur (fig. 18).

$\mathrm{Au}$ point de vue anatomique, le centre d'association postérieur occupe le précoin, une partie de la circonvolution linguale, la circonvolution fusiforme, toutes les circonvolutions pariétales, la circonvolution temporale inférieure et la partie antérieure de la face externe du lobe occipital.

Le centre d'association moyen correspond à l'insula de Reil.

Le centre d'association antérieur est constitué par la moitié antérieure de la circonvolution frontale moyenne, de la circonvolution frontale inférieure et 
par la circonvolution droite que l'on trouve sur la face inférieure du lobe frontal.

Chez l'homme, la zone des centres d'association occupe donc les deux tiers de la surface totale des hémisphères cérébraux.

En ce qui concerne leur structure, Flechsig admettait tout d'abord que les centres d'association sont complètement dépourvus de fibres de projection et par conséquent complètement indépendants des masses grises inférieures du névraxe, ils ne pourraient donc communiquer avec le monde extérieur, ainsi qu'avec les tissus et organes intérieurs du corps, que par l'intermédiaire des zones sensorielles, auxquelles ces dernières sont reliées par un nombre incalculable de fibres nerveuses. Il a dû reconnaître pourtant à la suite des travaux de Sachs, Dejerine, Monakow, etc., que les parties corticales qui constituent les zones d'association, sont pourvues d'un certain nombre de fibres de projection qui les mettent directement en rapport avec le système nerveux inférieur.

Quoi qu'il en soit, les différences fonctionnelles entre les sphères sensorielles et les sphères d'association ne sont pas niables. Les premières sont reliées au névraxe par de gros faisceaux de fibres nerveuses visibles à l'œil nu, tandis que les centres d'association ne sont pourvus que de rares fibres de projection qu'il faut chercher, pour les trouver.

Pour confirmer ses idées sur les différences fonctionnelles entre les centres sensoriels et les centres d'association, Flechsig rapporte, d'après Heubner, les deux observations cliniques suivantes:

$1^{\circ}$ La sphère auditive gauche entièrement intacte a été séparée de toutes les parties voisines de l'écorce 
situées au-dessus, en arrière et en dessous par un foyer de ramollissement. Le malade porteur de cette lésion, pouvait répéter tous les mots qu'on prononçait devant lui, mais n'en comprenait pas le sens et, de plus, il n'en gardait le souvenir que pendant quelques secondes. Le souvenir des images auditives et la valeur conventionnelle des mots se localisent donc en dehors de la sphère auditive, dans le grand centre d'association postérieur.

$2^{\circ}$ L'observation de Nothnagel : à la suite d'un ramollissement de la zone corticale de deux sphères visuelles survenu brusquement pendant la nuit, un homme devint aveugle. La destruction de ces sphères le met hors d'état de recevoir encore de nouvelles sensations visuelles. Malgré cela, cet homme conserve le souvenir de l'aspect extérieur des objets vus antérieurement et peut encore se représenter des images visuelles. Les images visuelles doivent donc se localiser dans le cerveau, au dehors de la sphère visuelle, dans la partie voisine du grand centre d'association postérieur.

Mais ce qui semble le mieux démontrer le rôle d'organe de la pensée des sphères d'association, c'est l'étude du développement du système nerveux et des facultés psychiques chez l'embryon humain.

D'après Flechsig, l'axe nerveux est formé exclusivement de substance grise jusque vers le milieu du cinquième mois de la vie embryonnaire. La substance blanche ne fait son apparition qu'à partir de cette époque et en comparant les embryons d'âges différents, Flechsig a constaté que cette apparition de la substance blanche se fait d'après un ordre déterminé et toujours le même, de sorte que, connaissant l'âge de l'embryon, on peut dire d'avance dans quel état 
de développement se trouvent les fibres nerveuses.

Il a constaté ensuite que les fibres qui ont les mêmẹs connexions anatomiques et, par conséquent, remplissent les mêmes fonctions physiologiques, développent leur myéline à la même époque, tandis que les faisceaux de fibres nerveuses ayant des connexions anatomiques différentes et qui sont d'une valeur fonctionnelle différente, prennent leur myéline à des époques différentes, de sorte qu'il a pu distinguer et suivre avec facilité et sans erreur possible les fibres à connexions anatomiques différentes dans leur trajet à travers le névraxe et établir leur origine et leurs terminaisons.

Flechsig a pu établir ainsi, que ce sont les fibres sensitives, c'est-à-dire centripètes qui arrivent les premières à l'état de maturité complète. Elles commencent à former leur myéline à partir du huitième mois de la vie intra-utérine et parmi ces fibres, les premières formées sont celles, qui relient les cordons postérieur's de la moelle avec les hémisphères cérébrales, et ensuite successivement les fibres olfactives, optiques et acoustiques. Comme ce sont les seules fibres complètement formées à ce stade de développement de l'embryon, on peut facilement suivre leur trajet et se convaincre qu'elles se terminent dans l'écorce grise des sphères tactile, olfactive, visuelle et acoustique.

Les fibres descendantes, centrifuges ou motrices ne commencent à se former qu'au moment où le développement des fibres sensitives est complètement achevé, c'est-à-dire après la naissance de l'enfant. Elles arrivent à maturité pendant le premier mois de sa vie.

Ainsi on peut considérer comme bien établi, que 
ce sont les excitations venant du milieu extériéur qui provoquent la formation des premières cellules réceptrices et des fibres nerveuses qui transmettent ces excitations à l'écorce cérébrale et que c'est sous l'action de ces excitations, que les cellules de l'écorce acquièrent le degré de développement nécessaire, pour provoquer à leur tour la formation complète des fibres descendantes, qui dirigent les réactions des organes.

"A ce moment, les centres d'association, formés uniquement de substance grise, sont encore complètement hors de fonction. »

Toutes les impressions tactiles, olfactives, visuelles, gustatives et acoustiques, conduites vers les sphères correspondantes de l'écorcre cérébrale, ne sont perçues que pendant le temps qu'elles durent. Dès qu'une de ces impressions cesse d'exciter l'organe périphérique, l'image cérébrale s'évanouit. Un enfant d'un mois ne peut répondre aux excitations du dehors que par des réflexes isolés, parce que ses centres sensoriels, complètement séparés les uns des autres par les centres d'association incomplets, ne communiquent pas encore entre eux. L'enfant d'un mois ne peut donc répondre aux excitation's du dehors que par des mouvements purement réflexes et se trouve ainsi, au point de vue fonctionnel, inférieur aux mammifères adultes complètement dépourvus des centres d'association, parce que chez ces derniers il y a entre les centres sensoriels des liaisons qui permettent la coordination de certains mouvements d'ensemble.

Il est facile de suivre chez l'enfant le développement parallèle, d'une part, des fibres qui relient les centres sensoriels aux centres d'association, ainsi qué Dunvsz. - La genèse de l'énergie psychique. 
ces derniers entre eux et, d'autre part, le développement progressif de son intelligence. Ces fibres commencent à se myéliniser au commencement du deuxième mois après la naissance. Elles se rendent dans l'écorce grise voisine de la sphère sensorielle à laquelle elles appartiennent. "Là, dit Van Gehuchten, d'après Flechsig, va se former un nouveau centre, où sera conservé le souvenir des impressions tactiles, visuelles, olfactives ou acoustiques. L'enfant commencera à reconnaître, à partir de cette époque, les impressions déjà ressenties antérieurement. Les fibres d'association pénétreront plus avant encore dans les sphères intellectuelles: les unes relieront directement les sphères sensorielles entre elles : soit la sphère auditive à la partie inférieure de la sphère tactile: l'enfant pourra répéter les mots entendus, mais sans les comprendre; les autres se termineront dans les sphères intellectuelles : il se formera là des centres où vont se rencontrer des fibres venant de la sphère visuelle, de la sphère auditive et de la sphère tactile: les impressions visuelles, tactiles et auditives pourront être comparées entre elles et l'enfant commencera à saisir la signification des objets du monde extérieur. "

La formation et la myélinisation des fibres nerveuses d'association, ainsi que la formation des centres d'association secondaires va se poursuivre pendant longtemps jusqu'à l'âge adulte et probablement au delà, mais on trouvera là, très probablement, des différences de race et des différences individuelles considérables. C'est là un territoire encore bien peu exploré en ce moment, mais on peut admettre la durée plus ou moins longue du développement des centres nouveaux, en constatant l'arrêt plus ou moins 
précoce ou tardif des facultés psychiques chez les hommes appartenant aux différentes races et chez différents individus de la même race. Pour ne prendre comme exemple, que les extrêmes, on constate chez les nègres d'Afrique un arrêt du développement de l'intelligence à un âge bien plus bas (12 à 16 ans) que chez les blancs et on peut dire que chez ces derniers les différences individuelles sont tellement considérables qu'il est presque impossible d'assigner à ce développement une limite quelconque.

Chez les hommes de race blanche et jaune, on peut trouver, en effet, de nombreux exemples de l'apparition des facultés cérébrales nouvelles à tout âge et il n'est pas douteux que l'apparition de ces idées d'une orientation ou d'une puissance nouvelles doive correspondre à la différenciation des centres d'association secondaires nouveaux et de connexions nouvelles.

Voilà à peu près tout ce que l'on peut dire aujourd'hui de la structure générale du pallium de l'homme et des fonctions des différentes sphères qu'on peut $\mathrm{y}$ distinguer.

Nous avons vu plus haut que les sphères d'association occupent chez l'homme à peu près les deux tiers de la masse totale du pallium; chez les singes anthropoïdes, l'ensemble de ces sphères n'en occupe que la moitié; chez les mammifères supérieurs, un tiers, un quart; chez les rongeurs et chez tous les vertébrés inférieurs, on n'en trouve plus du tout.

Il faut reconnaître que c'est un territoire qu'il y aurait grand intérêt à explorer avec beaucoup de soin et de précision, que cela n'a été possible de le faire jusqu'à présent, et il est certain qu'on y reviendra quand les énergies intellectuelles absorbées aujourd'hui en grande majorité par les sciences 
physico-chimiques, éprouveront de nouveau le besoin de se retremper dans l'étude plus ingrate de l'anatomie et de l'histologie et d'y rechercher des bases positives d'idées nouvelles.

Toutefois, de ce que nous en savons aujourd'hui, on peut déjà tirer des aperçus généraux fort intéressants et suggestifs, que ceux qui nous ont suivis jusqu'à présent résumeront sans doute de la façon suivante :

Le tissu nerveux apparaît chez les cœlentérés, sous forme d'organes des sens rudimentaires (fig. 1) et de là il évolue:

D'une part, vers une formation de chaînes ganglionnaires avec prédominance marquée du système des ganglions ventraux, qui président à la vie réflexe et qui atteint son maximum de développement chez les insectes, développement relativement supérieur à celui du même système chez les vertébrés et chez l'homme;

D'autre part, en passant par les tuniciers, vers la formation de chaînes ganglionnaires avec prédominance de plus en plus marquée du système des ganglions dorsaux (cérébro-spinal) et enfin du cerveau proprement dit dont l'évolution progressive peut être suivie pas à pas depuis l'amphioxus jusqu'à l'homme (fig. 7 à 25).

Le cerveau, une fois formé, on constate un accroissement progressif et constant de sa partie terminale, du pallium qui devient d'abord le centre dominant des réactions sensorielles purement réflexes et ensuite, par une série de différenciations intimes, de plus en plus nombreuses et délicates, le centre d'association, c'est-à-dire de combinaison et de coordination des réactions réflexes enregistrées par les 
centres sensoriels, tel que nous le trouvons aujourd'hui chez l'homme civilisé.

2. Développement parallèle du tissu nerveux et des énergies nerveuses. - Parallèlement au développement anatomique et à la différenciation de plus en plus délicate du tissu nerveux, on constate le développement progressif de l'énergie nerveuse et cérébrale.

Aux réflexes purement tropiques et locaux chez les cœlentérés, succèdent, avec l'apparition des fibres et des ganglions nerveux, d'abord des réactions d'ensemble de mieux en mieux coordonnées, mais encore purement réflexes, et ensuite, avec la formation des centres d'association, des réactions raisonnées.

Est-il possible de délimiter nettement ces trois stades de l'évolution de l'énergie nerveuse, de trouver l'animal complètement dépourvu de la faculté de coordonner ses réflexes, et celui qui la possède déjà, peut-on désigner avec certitude l'animal qui a commencé le premier à réfléchir et à penser ?

Certainement non !

La méduse, l'actinie, exécutent certains mouvements d'ensemble sans posséder un centre de coordination différencié, et on trouve parmi les rongeurs des espèces qui, malgré l'absence complète de sphères d'association distinctes, font preuve de plus d'intelligence que certains carnassiers qui possèdent déjà des sphères d'association assez nettement délimitées.

La faculté de former des idées associées et de les exprimer, en un mot, de penser d'une façon consciente n'appartient réellement qu'à l'homme, mais entre l'intelligence d'un savant, d'un sociologue ou d'un artiste de premier ordre et celle d'un sauvage 
le plus primitif, n'y a-t-il pas une différence plus grande, qu'entre ce dernier et le chimpanzé ?

En suivant le développement des facultés psychiques chez l'homme à partir de sa naissance, on constate d'abord l'apparition de la mémoire réflexe, ensuite de la mémoire consciente, puis de la faculté de comparer, de combiner et de coordonner les impressions fixées par la mémoire et enfin celle de réagir à ces impressions, de les exprimer ou de les réaliser par des conceptions ou des actes individuellement volontaires sinon arbitraires, en un mot, de penser et d'agir en poursuivant des buts plus ou moins bien définis suggérés par des impressions venues du milieu extérieur et intérieur de l'organisme et qui se sont imprimées sur l'écorce cérébrale d'une façon consçiente ou inconsciente.

Les loups qui s'entendent à plusieurs pour organiser de véritables parties de chasse, dans lesquels les uns se mettent à l'affût, tandis que d'autres font les rabatteurs; le chien qui a appris à se défaire du collier qui le tient attaché à sa niche et à se le remettre lui-même après une fugue qu'il sait défendue, font preuve de réflexion, de pensée consciente. Les carpes qui viennent en foule se ranger au bord du bassin à l'approche d'un homme, savent nécessairement ce qui les attend et témoignent ainsi de mémoire consciente et d'un rudiment de réflexion leur permettant de saisir les relations entre les causes et les effets des phénomènes qui les intéressent.

Il est inutile de multiplier ici les exemples de la sensibilité consciente ou de l'intelligence des bêtes, que de nombreux écrits ont suffisamment popularisés, pour nous convaincre que, s'il y a aujourd'hui 
des lacunes considérables entre les degrés de l'échelle du développement du tissu nerveux des animaux qui existent actuellement et des fossiles, par exemple, entre les cœlentérés et le balanoglossus, entre ce dernier et les poissons, une filiation dans l'évolution de ce tissu, une progression continue dans le volume, la complexité et la différenciation de ce tissu depuis les protozoaires jusqu'à l'homme, est indéniable.

La formation du tissu nerveux a son origine dans la sensibilité générale de la matière vivante aux agents physiques et chimiques du milieu extérieur; dans les excitations qui se traduisent par des modifications de composition chimique et de structure intime, qui, à leur tour, ont pour conséquence la différenciation, la sélection et enfin l'organisation des éléments les plus actifs en cellules, fibres et centres nerveux.

Comme pour toutes les unités matière-énergie qui ont précédé la formation de la cellule vivante, l'évolution du tissu nerveux d'abord puis cérébral, d'une part, de formes d'énergie nerveuse et cérébrale de plus en plus variées et puissantes, d'autre part, est la conséquence directe d'une série ininterrompue de réactions consécutives et alternantes entre les éléments sélectionnés et réunis en unités distinctes de plus en plus complexes et les formes d'énergie nouvelles de plus en plus puissantes qui en procèdent.

On peut résumer cette évolution par le tableau suivant : 
Cellule vivante

Cellules nerveuses périphériques

Ganglions ou centres nerveux correspondants

Connexions entre plusieurs centres sensoriels

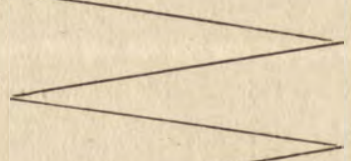
Sensibilité générale réactions tropiques

Sensibilité différenciée réactions réllexes

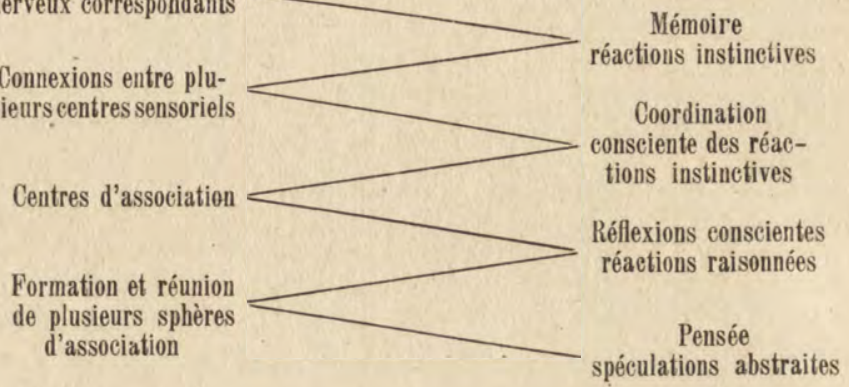

Y a-t-il une raison valable de supposer que cette évolution progressive, dont nous avons parcouru les étapes depuis l'éthéron, s'arrêtera à l'homme tel qu'il est aujourd'hui ? L'idée que l'homme tel qu'il est, ne serait passible que d'un perfectionnement moral est encore actuellement la base fondamentale de toute notre philosophie et les plus folles imaginations des conteurs, qui se sont transportées par la pensée dans l'avenir, n'ont su nous représenter l'homme futur que comme un mécanisme de plus en plus perfectionné, ou dégoûté de tout progrès mécanique, mais guère plus riche en puissance nouvelle, que nous le sommes à ce jour.

Pour les astronomes, qui se sont amusés à vaticiner, l'homme, issu de la terre et attaché à elle, devra nécessairement finir avec elle ou plutôt il périra dégénéré, revenu à la vie de cavernes sur une terre, dont toute la surface sera recouverte d'un 
rocher, formé par l'eau complètement solidifiée et aussi dur que du granit. Les radiologistes nous promettent, il est vrai, que la chaleur de la terre sera maintenue au même point par le rayonnement des corps radioactifs ou même augmentée jusqu'à une température qui la fera revenir à l'état de gaz dont elle est sortie.

Quoi qu'il en soit de ces deux modes d'anéantissement par le froid ou par une chaleur excessive, on n'a prévu jusqu'à présent d'autre avenir pour la vie ou la matière vivante sur cette terre, qu'une destruction totale, de sorte que, pour les penseurs, pour lesquels l'idée d'une âme immortelle ou, en d'autres termes, d'une énergie métaphysique, ne repose sur aucun fondement raisonnable, tous les efforts de notre énergie vitale n'auraient d'autre raison d'être que de poursuivre les misérables buts plus ou moins immédiats de la vie courante.

Rien, en effet, n'est plus décourageant et démoralisant qu'un travail sans but, et la satisfaction à des nécessités immédiates ne peut devenir une source d'énergie nouvelle et féconde que s'il nous est possible de voir au delà, des buts plus lointains.

Or, la science est arrivée à un tournant qui lui permet de prévoir un avenir moins désespérant.

Les pronostics les plus pessimistes, basés sur ce que nous savons de son passé, nous permettent de prévoir pour la terre encore une existence d'au moins quelques milliers de siècles, dans des conditions qui ne seront pas très sensiblement diftérentes de celles, dans lesquelles nous vivons actuellement.

Que deviendra alors, quelles transformations peut subir le cerveau de l'homme actuel pendant ces milliers d'années?

Danysz. - La genèse de l'énergie psychique. 
Suivons encore une fois l'évolution du pallium depuis le stade poisson jusqu'à celui de l'homme actuel.

Nous voyons qu'il y a là une progression continue.

Peut-on alors admettre raisonnablement que le cerveau de l'homme restera définitivement stabilisé dans son volume, sa forme et sa structure actuels?

Tout nous porte à croire que non, qu'au contraire, son évolution progressive sera de plus en plus rapide, parce que le développement de la conscience, qui devient chaque jour plus délicate et embrasse des champs chaque jour nouveaux et plus vastes, influera sur le développement de nouveaux centres de perception et d'association qui, à leur tour, augmenteront la puissance de l'ensemble de l'énergie psychique.

Et quand on vient à comparer la durée des périodes du progrès depuis les temps historiques et ce que nous savons des temps préhistoriques, on en arrive forcément à conclure, que cette progression sera de plus en plus rapide.

On trouve des hommes remarquablement intelligents dans l'antiquité égyptienne, chinoise, grecque, et on veut en conclure que si, au point de vue de son développement psychique, l'homme n'a pas fait de progrès sensibles depuis trois ou quatre mille ans, il n'y a pas de raison de supposer qu'il en fera dans l'avenir. Un tel argument, qui pouvait encore être admis par les savants de la génération qui nous a précédé, ne résiste plus à une critique basée sur les faits connus aujourd'hui.

On admettait alors que les premiers hommes ont apparu sur la terre il y a dix mille, tout au plus quelques dizaines de millénaires. Aujourd'hui, on sait d'une façon certaine, par la découverte de mâchoires 
et de crânes humains de Nauen (près de Heidelberg), de Neanderthal (près de Elberfeld), de La Chapelle (Somme), que l'homme existait déjà pendant et avant les grandes périodes glacières. Des squelettes presque entiers trouvés dans les grottes de Grimaldi (près de Menton), dans la Dordogne, la Charente, à Gibraltar, en Belgique, en Bohême, en Angleterre, prouvent que déjà à cette époque lointaine, l'homme était répandu dans toute l'Europe, et la forme de son crâne, fortement aplati dans la région frontale, avec des arcades sourcilières très saillantes, fait penser que son pallium devait être nécessairement moins développé que celui d'un homme de la race la plus inférieure d'aujourd'hui.

A en juger par l'âge probable des formations géologiques dans lesquelles on trouve des débris humains, on doit admettre que la race d'homme, appelée race de Neanderthal, a vteu au moins cent à cent cinquante mille ans avant notre époque historique. L'industrie de ces hommes, c'est-à-dire leur intelligence, était en rapport avec le développement de leur cerveau. Les silex taillés en sont les seuls témoignages.

Les preuves d'un développement cérébral et d'une intelligence sensiblement plus avancés ont été trouvés par Louis Lartet, dans la grotte de Cro-Magnon, dans la Dordogne, sur les bords de la Vézère. Les parois de cette grotte sont couvertes de dessins gravés dans la pierre, de peintures et des sculptures représentant les hommes ainsi que les animaux : rennes, bisons, mammouths, rhinocéros, lions, qui peuplaient ces régions à cette époque. On y trouve même des tableaux représentant des chasses et des batailles, mais comme preuves de l'industrie, uniquement en- 
core des outils taillés dans la pierre, les cornes et les dents des mammouths.

Les hommes de cette époque, la race de renne, ont vécu, selon toute probabilité (Marcelin Boule) environ vingt mille ans avant l'époque historique. Ils ont laissé des traces de la connaissance de l'utilisation du feu, mais pas encore du travail d'un métal quelconque.

Nous avons tenu à reproduire ces faits, d'après les documents les plus récents, pour faire ressortir :

D'une part, que trente ou quarante siècles sont une durée bien faible pour produire des modifications anatomiques et histologiques notables dans le cerveau pour nos moyens d'investigations actuels et, par là, dans les facultés psychiques de l'homme.

D'autre part, que la durée des époques par lesquelles on peut marquer les progrès dans l'activité intellectuelle de l'homme, ou, en d'autres termes, que les durées des étapes successives de l'évolution progressive du tissu cérébral et des énergies qui en émanent, sont de plus en plus courtes.

Il a fallu 150.000 ans pour arriver de l'homme du Neanderthal, qui connaît déjà le feu et sait tailler le silex, à l'homme du Cro-Magnon, qui ne connaît pas encore l'usage des métaux, une vingtaine de mille pour arriver de là aux premiers documents écrits et des milliers, puis seulement des centaines d'années, pour accomplir tous les progrès qui marquent les étapes successives de l'époque historique.

Et quand on cherche à se rendre compte de quelle nature pouvaient être les agents de cette évolution progressive de l'homme, on trouve que ce sont les découvertes des instruments, appareils et méthodes qui, en augmentant la puissance d'action de ses sens naturels, ont rendu l'exécution de tous les travaux plus 
rapide, plus facile et plus sûre et ont dû réagir par là, parallèlement, sur le développement en volume et en différenciation de plus en plus délicate des centres sensoriels et des centres d'association d'une part, de la conscience et de la faculté de coordination des idées, d'autre part.

La découverte du feu et son utilisation comme source d'énergie calorique et lumineuse, des propriétés des métaux, de la balance, des lentilles grossissantes, de l'électricité, de la vapeur, des lois de chimie et de physique, de systèmes et méthodes de plus en plus sûres de recherches et de travail, des moyens de transport facilitant les communications entre les hommes de différentes races, en un mot les découvertes concernant des faits précis qui consti-tuent la charpente solide et toujours perfectible de notre savoir, ont eu et auront toujours une influence infiniment plus considérable sur le développement de l'énergie psychique et de son organe, que les spéculations philosophiques d'ordre politique, moral ou social. Autant qu'il est possible d'en juger par les documents des temps historiques, ce genre de spéculation doit plutôt être considéré comme un frein, un agent de décadence, que de progrès.

Le système d'organisation et d'exécution du travail découvert et introduit dans la pratique par l'ingénieur américain Taylor, fera certainement bien plus pour le bonheur et le développement intellectuel de l'ouvrier que le système de Marx appliqué par Lénine.

La découverte du piano, celle du microphone ou du microscope ou la co:nbinaison d'un orchestre contribuera avec certitude au développement des centres auditif et visuel de l'écorce cérébrale et par là au 
développement parallèle des centres d'association correspondants, et il est non moins certain que le système de Bouddha ou de Mahomet n'a pu y contribuer en aucune façon et a marqué au contraire un temps d'arrêt dans ce développement, en remplaçant chez l'homme les désirs d'activité créatrice de constructions réelles par des visions purement imaginaires et nécessairement stériles.

La philosophie a pourtant un vaste champ d'activité créatrice ouverte devant elle, et cette activité sera féconde au lieu d'être néfaste, à condition d'abandonner les chimères d'imagination pure et de se consacrer à l'interprétation de plus en plus exacte, à la généralisation et à la synthèse des faits et des phénomènes connus.

Aristote, Euclide, Galilée, Kopernik, Newton, Diderot, Lamarck, Darwin, Pasteur, Hertz, Curie, Virchof, Spencer, Loeb, Einstein, etc., ont contribué bien plus au progrès général de l'humanité que Confucius, Kant ou Hegel, et il est intéressant de faire remarquer ici, à titre d'exemple frappant, que si le travail, bien organisé et adapté au développement des connaissances réelles, a fait de l'Allemagne, en quelques dizaines d'années, un des peuples les plus puissants de la terre, c'est la généralisation par l'instruction des principes énoncés par ses philosophes (Hegel, Fichte, etc.), qui l'a conduite à une catastrophe dont elle ne se relèvera peut-être jamais complè-. tement.

Ainsi, c'est l'étude des phénomènes réels qui impressionnent nos sens, ainsi que des objets, tissus, organes du milieu extérieur et intérieur, dont nous pénétrons de mieux en mieux la structure intime, la composition et les fonctions à l'aide de nos instru- 
ments et de nos méthodes de recherches expérimentales qui, en augmentant la puissance et la délicatesse de notre conscience, provoquera la formation des centres de perception et d'association nouveaux dans le pallium.

Et connaissant l'histoire de l'évolution du pallium depuis l'époque où il s'est formé chez les premiers poissons jusqu'à celle de l'homme civilisé d'aujourd'hui, nous pouvons affirmer avec certitude:

$1^{\circ}$ Que le pallium continuera à se développer ;

$2^{\circ}$ Que ce sont principalement les sphères d'association qui s'enrichiront de centres nouveaux;

$3^{\circ}$ Que cette évolution sera de plus en plus rapide, parce que chaque découverte a généralement pour résultat d'ouvrir des horizons nouveaux à la recherche de plusieurs autres.

On peut prévoir l'arrivée d'un moment où la différence entre le cerveau de l'homme civilisé d'aujourd'hui et celui de l'homme futur ou plutôt de certains individus ou races d'hommes futurs, sera à peu près la même que celle que nous constatons actuellement entre le pallium de l'homme et celui d'un singe, d'un chien et peut-être même du poisson. Et alors quelles seront les facultés psychiques nouvelles, dont ce pallium futur sera l'organe- Tout ce qu'on peut en dire aujourd'hui, c'est que la puissance de l'énergie cérébrale sera alors formidable!

Il y a là de quoi exercer l'imagination des poètes, mais le moment n'est probablement pas très éloigné où les continuateurs des beaux travaux de Cajal, Van Gehuchten, Dejerine, Nageotte, Flechsig His, Bechteref, Marinesco, Monakow, Minkowski, etc., montreront la structure intime du pallium, la différenciation et les fonctions des différents centres qui la 
composent, d'une façon suffisamment précise, pour qu'il soit possible de prédire avec une probabilité suffisante dans quel sens l'accroissement de l'énergie psychique sera dirigé tout d'abord.

Actuellement, tout ce que nous pouvons en dire, c'est que l'évolution progressive constatée jusqu'à présent nous oblige à admettre la continuation de cette évolution à l'infini, parce que la puissance du facteur dominant de cette évolution, de l'unité matière-énergie: cerveau-pensée, s'accroît continuellement et devient par cela même illimitée.

3. L'immortalité. - Avant de clore ce chapitre, il nous semble encore nécessaire de soumettre à la discussion et à une analyse aussi objective que possible une question qui préoccupe beaucoup d'esprits éminents, la question de l'âme, telle que la conçoivent les croyants et telle qu'elle peut être pour les biologistes.

Pour les croyants, l'existence d'une âme immortelle est une profession de foi, qui échappe par cela même à toute discussion et nous avons vu que, pour le biologiste-philosophe, nier l'existence d'une énergie psychique consciente, qui a pour origine et pour organe le cerveau de l'homme, d'une énergie, pour laquelle nous avons été obligés d'admettre une évolution illimitée, c'est-à-dire éternelle, serait nier l'évidence.

Sur ce point, sur la question de principe, il y a donc un accord parfait. Les difficultés, ou plutôt les malentendus commencent quand on arrive à examiner les questions de détail concernant l'origine et 
là nature de cette âme-énergie, qui pourtant repose sur la question primordiale de savoir s'il est possible d'admettre l'existence d'une "énergie en soi ", ce qui en définitive reviendrait à dire d'un " mouvement en soi ». Posée de cette façon, cette question trouve donc sa réponse en elle-même. Nous avons vu, en effet, en définissant les unités matière-énergie, qu'un mouvement ne peut être que considéré comme l'effet produit par une particule matérielle, d'une unité de matière en mouvement, que le mouvement est une propriété indissoluble de la matière, que toutes les formes d'énergie les plus complexes et les plus subtiles ne sont et ne peuvent être que des effets ou des manifestations des mouvements combinés de particules matérielles successivement sélectionnées parmi les plus actives et réunies elles-mêmes en complexes de plus en plus compliqués.

Les malentendus entre les conceptions chrétiennes sur l'origine et la nature de l'âme, telles qu'elles s'imposent encore aujourd'hui sous forme de professions ou d'articles de foi aux peuples civilisés, et les conceptions biologiques sur l'origine et la nature de l'énergie psychique, ont pour cause unique le fait, que les premières avaient été conçues et formulées par des cerveaux de vingt siècles plus jeunes, par des cerveaux qui ne savaient pas, ne pouvaient pas savoir ce que nous savons aujourd'hui et n'étaient pas pourvus de tous les centres de perception d'association dont ils se sont entrichis depuis.

Pour le biologiste d'aujourd'hui, admettre l'existence d'une âme ou, en d'autres termes, la production de la pensée, sans l'existence d'un cerveau vivant qui la produit, équivaudrait à admettre l'existence d'une affinité chimique sans molécules chimiques 
pour un chimiste, de l'électricité, de la lumière ou de la chaleur sans électrons ou atomes, pour un physicien.

Et il n'est pas sans intérêt de noter ici combien certains prêtres, gardiens instruits de la foi chrétienne, ont progressé dans le sens de la conception d'une évolution de l'âme. Le rév. Canon Barnes admet dans un prêche, dont nous trouvons un résumé dans le Daily News de Londres du 17 janvier 1921, quatre stades ou périodes dans l'évolution de l'âme humaine à partir des organismes dépourvus d'âme. Ce seraient :

$1^{\circ}$ La période de l'action instinctive;

$2^{\circ}$ La période de perception du monde extérieur;

$3^{\circ}$ De l'action intelligente;

$4^{\circ}$ De conception abstraite, idéale.

N'est-il pas évident que le moment est proche où la religion, ou plutôt les prêtres, de plus en plus instruits (condition indispensable pour maintenir le contact spirituel et exercer une influence bienfaisante sur leurs fidèles), seront obligés de faire encore quelques pas dans les deux sens de cette évolution, dont ils admettent déjà un tronçon, pour l'instant isolé? Qu'ils seront forcément amenés à chercher d'une part l'origine de l'action instinctive dans la sensibilité générale de la matière vivante, celle-ci dans les affinités colloïdale et moléculaire, et ainsi de suite jusqu'à l'énergie primordiale qui est probablement l'attraction universelle; d'autre part, à se convaincre que l'âme de l'homme actuel ne peut être autre chose que la dernière, la plus parfaite et la plus puissante forme de cette énergie universelle et de trouver ainsi l'explication réelle et si simple de son immortalité par sa perfectibilité infinie?

\section{http://rcin.org.pl}


Et ne serait-il pas plus consolant pour nous, simples mortels, et surtout plus fécond pour notre activité, de penser que les efforts de l'intelligence, dont nous avons quelques raisons d'être fiers quelquefois, ne finiront pas sans retour avec les dernières gouttes d'eau solidifiées ou le dernier grand feu qui transformera la terre en vapeurs; mais qu'ils contribuent à cette évolution progressive qui permettra à l'homme futur de maîtriser la nature au point de prévoir les changements cosmiques possibles et d'en modifier le cours à sa convenance, que de croire à une âme immortelle, mais condamnée à cette existence éternelle sans but, tant soit peu défini?

4. Télépathie. - Encore quelques mots sur un objet qui n'est pas encore entré dans le domaine de la science expérimentale proprement dite, mais qui mérite une analyse, parce qu'il préoccupe beaucoup d'esprits et, parmi eux, ceux de plus d'un savant de premier ordre (Crookes, Ch. Richet, Oliver Lodge, etc.). Il s'agit de télépathie.

Nous avons vu dans les chapitres précédents, que les différentes formes d'énergie que nous connaissons peuvent être rangées dans deux grandes classes :

$1^{\circ}$ Les énergies rayonnantes, telles que l'attraction universelle, l'électricité, la lumière, la chaleur, le son, qui peuvent agir à distance, ce qui veut dire qui rayonnent dans l'espace, peuvent être transmises d'un endroit à un autre;

$2^{\circ}$ Les énergies que nous avons appelées $d u m i$ lieu intérieur, caractérisées par le fait qu'elles ne rayonnent pas dans l'espace et n'agissent pas à distance (appréciable). 
De ces dernières, nous n'en connaissons, à propre ment parler, qu'une seule assez bien, pour pouvoir en provoquer les manifestations à volonté, c'est l'affinité chimique.

Dans laquelle dé ces deux classes allons-nous placer l'énergie psychique?

Il a été impossible jusqu'à présent de provoquer à volonté le passage direct d'une pensée ou d'une impression nerveuse quelconque d'un individu quelconque à un autre. On ne le peut que par l'intermédiaire des organes des sens. On sait aussi que dans le milieu intérieur du corps, une manifestation de la volonté, un mouvement volontaire quelconque ne peut se produire que si le centre de la volonté est relié à l'organe ou le tissu qui doit être mis en mouvement par un ou une série de filets nerveux spéciaux. Il nous serait impossible d'exécuter volontairement un -mouvement quelconque, de parler ou d'écrire, si les filets moteurs qui relient la langue ou la main aux centres moteurs étaient coupés ou bien si ces centres eux-mêmes étaient paralysés.

Pour que la transmission directe de la pensée d'une personne à une autre soit possible, il faudrait admettre la production et l'émission par le cerveau d'ondes psychiques comparables aux ondes électriques de Hertz, qui servent à la télégraphie sans fil et ceux qui croient à la transmission de la pensée soutiennent que, puisque personne ne pouvait se douter de l'existence des ondes hertziennes avant la découverte des appareils qui les émettent et qui les enregistrent (ce qui n'est d'ailleurs pas tout à fait exact, parce que Maxwell a prévu l'existence de ces ondes par des déductions théoriques), on pourrait en dire autant des ondes psychiques, pour 
lesquelles certaines personnes seulement ont le privilège de posséder des organes émetteurs ou enregistreurs.

Un tel raisonnement, il est à peine besoin de le dire, ne résiste pas à la critique. Personne, jusqu'à présent, n'a manifesté le pouvoir d'émettre ou d'enregistrer à volonté les ondes psychiques, ce qui seul indiquerait l'existence préalable d'organes émetteurs et enregistreurs de ces ondes. Ces phénomènes, autant qu'on peut en juger par les récits de ceux qui les ont éprouvés, se manifestent brusquement, généralement une seule fois. Pour en admettre la réalité, il faudrait donc admettre avant tout la possibilité de la formation brusque, sans aucune cause apparente de ces organes spéciaux.

Dans son livre intitulé Raymond, Sir Oliver Lodge décrit une série de communications qu'il a eues avec son plus jeune fils, tué à la guerre. Ce fils manifeste sa présence par toutes sortes de moyens détournés, des tables tournantes, des coups frappés sur les portes ou les murs, des "médiums "... et pourtant, si l'âme était l'essence même de la pensée et pouvait se déplacer dans l'espace, comme on l'admet généralement dans le monde des spirites, est-ce que cette âme de Raymond aurait besoin d'un pied de table et de tous ces petits subterfuges pour se manifester dans la pensée consciente de son père?

Inutile, je pense, d'insister sur l'absence de tout fondement scientifique concernant la transmission directe de la pensée. Nous ne savons pas ce que nous réserve l'avenir sous ce rapport. Il arrivera peut-être un moment où l'homme sera pourvu d'organes d'émission et d'enregistrement des ondes psychiques; pour le moment, il serait beaucoup plus profitable 
d'approfondir nos connaissances sur la production et le passage dans les fibres des courants nerveux dont nous constatons l'existence réelle. Il est possible d'admettre, en effet, que l'homme parviendrait peutêtre à modifier, dans un sens qui lui serait utile, la structure et le fonctionnement de certains organes, s'il en connaissait exactement le mécanisme le plus intime, s'il savait avec une précision suffisante ce qu'il faudrait vouloir, non seulement en gros, mais en détail.

Toutefois, s'il est impossible de nier en bloc les manifestations télépathiques, il s'agit d'en expliquer le mécanisme; mais la tâche n'est pas facile, parce qu'on ne dispose jamais de tous les éléments, de toutes les données qui composent ce problème et dont l'appréciation serait indispensable pour le résoudre d'une façon impartiale.

Tout ce qu'on peut en dire tout d'abord, c'est que ce sont surtout les personnes facilement suggestionnables, tant par d'autres personnes que par ellesmêmes, qui ont éprouvé ces manifestations, et on sait combien fortes sont quelquefois certaines illusions. Ensuite, il faut tenir compte d'un certain travail dont nous n'avons pas conscience et dont le cerveau peut être le siège. Un exemple d'un tel travail a été donné par Henri Poincaré à l'une de ses conférences aux Sociétés Savantes.

Le savant mathématicien raconte, qu'il a eu brusquement la révélation de la solution d'un problème de mathématique, auquel il avait travaillé sans succès deux ans auparavant et auquel il n'a jamais plus réfléchi pendant ces deux années. La révélation s'est produite d'une façon aussi brusque qu'inattendue, au moment où, appelé à faire son service 
militaire, il montait dans le train avec quelques camarades.

Le cerveau peut donc non seulement retenir les impressions reçues, mais il peut les modifier et même les faire évoluer dans un sens précis et ne les faire apparaître dans la conscience, qu'au moment où elles sont suffisamment mûres, après une sorte d'incubation plus ou moins longue.

Il est donc possible d'admettre, que des impressions de toutes sortes peuvent subir des incubations analogues et que les manifestations soi-disant télépathiques ne seraient que des révélations qui, quand elles coïncident plus ou moins avec un fait concernant une personne qui nous intéresse, nous donnent l'illusion d'une transmission psychique.

C'est de cette façon, par un véritable processus de l'inconscient, que Flournoy, l'éminent philosophe de Genève, cherche à expliquer les "révélations" d'un de ses médiums, qui croyait communiquer avec les habitants de la planète Mars. Et il nous semble intéressant de faire remarquer ici, que dans la plupart de cas analogues cités par les instituts des sciences psychiques, il ne peut être question d'une transmission directe de la pensée, telle que nous l'avons définie: une énergie du milieu intérieur, non rayonnante.

Nous avons vu, en effet (tableau, p. 80), que la pensée procède, par une série d'étapes successives, de la sensibilité générale, et cette dernière de l'affinité chimique, et nous avons vu par ailleurs que ces formes d'énergies ne sont pas transmissibles.

Nous devons donc admettre, jusqu'à nouvel ordre, que la pensée ne peut être éveillée que par des impressions sensorielles indirectement transmises du milieu extérieur ou par des réminiscences incubées 
de ces mêmes impressions antérieurement enregis. trées. Ce qui donne l'apparence de spontanéité, c'est le fait, que ces impressions ont pu se produire dans un passé très lointain, qu'elles peuvent même être transmises par hérédité.

Je ne me flatte pas d'avoir convaincu par ces arguments les vrais croyants à la réalité des phénomènes de la télépathie, mais je ne les crois pas inutiles, comme indication de quelques données de ce problème, pour ceux qui voudraient essayer de le résoudre.

5. Résumé. - Pour résumer les faits et les idées contenus dans ce chapitre, il faudrait donc retenir :

$1^{\circ}$ Que dans l'évolution de la matière vivante, la formation des cellules et du tissu nerveux différencié a constitué une première étape d'une importance décisive dans l'évolution progressive. La sensibilité nerveuse a été, en effet, le seul facteur déterminant du perfectionnement graduel du corps des invertébrés et des vertébrés inférieurs en vue d'une adaptation de plus en plus parfaite au milieu extérieur, par la délicatesse et une coordination de plus en plus subtiles des réactions réflexes.

$2^{\circ}$ Que tant que dans l'être vivant formé par une série de segments (metamères) il ne s'est formé, dans aucun de ces segments, un centre nerveux dominant nettement l'ensemble de tous les autres centres, l'énergie nerveuse ne pouvait se manifester que par des réactions bien plus réflexes que réfléchies.

$3^{\circ}$ Que la formation d'un cerveau dominant nettement, par son volume et par la complexité de sa structure, l'ensemble de tous les autres centres nerveux, ainsi que la formation des sphères d'associa- 
tion, qui ont eu pour conséquence l'apparition de l'énergie psychique, a constitué un nouvel échelon d'une importance décisive de l'évolution progressive, en permettant à l'homme d'intervenir d'une façon consciente dans l'adaptation du milieu extérieur à ses besoins.

Les deux grandes périodes de l'évolution des êtres vivants déterminées par l'évolution du tissu nerveux seraient done :

La première : La période d'adaptation des êtres vivants au milieu extérieur dans lequel ils sont obligés de vivre, par l'énergie nerveuse réflexe.

La deuxième : La période d'adaptation du milieu extérieur à la vie de l'animal par l'intervention de l'énergie psychique consciente.

\section{9. - Règles générales de l'évolution}

En résumant les données que nous venons d'analyser, on peut donc affirmer que les règles générales de la succession des synthèses des unités "matièreénergie " sont déterminées:

1. Par les variations des conditions de milieu, c'est-à-dire de la température et de la pression qui, agissant en sens contraire l'une de l'autre, s'équilibrent à un moment donnée et ne peuvent pas dépasser un certain " maximum ".

L'élévation progressive de la température et de la pression est déterminée par la condensation des unités de la matière, probablement sous l'action de l'attraction universelle; le "maximum " de température et de pression est déterminé par la quantité de matière qui se condense dans un espace déterminé. A partir de ce "maximum ", il y a abaissement pro-

DAnYsz. - La genèse de l'énergie psychique. 
gressif de la température et de la pression par rayonnements et par projections dans l'espace;

2. Par une loi de structure, dont nous ignorons la nature, mais dont nous constatons l'intervention par ce fait, que, dans tout l'univers accessible à nos investigations, il se forme des espèces d'unités primordiales (électrons, atomes, molécules) d'une structure identique;

3. Par une sélection, qui est elle-même fonction de la structure et qui a pour résultat de ne faire participer aux synthèses successives, déterminées par les conditions de milieu, que les unités les moins stables et par là les plus actives, qui déterminent, à leur tour, la nature et les propriétés caractéristiques des unités "matière-énergie " d'un ordre plus élevé, qui en dérivent.

La loi générale de l'évolution des unités " matièreénergie " que l'on peut faire ressortir de cet ensemble de faits et de considérations que nous pouvons résumer et formuler avec une précision suffisante serait donc :

Les unités matière-énergie d'un ordre quelconque ne sont formées et ne peuvent être formées que par le travail organisé résultant de la réunion et de l’organisation en unités de travail distinctes des unités de. travail de l'ordre immédiatement précédent.

\section{0. - Les grandes périodes de l'évolution}

L'évolution générale peut être divisée en trois grandes périodes caractérisées par des conditions de milieu différentes et par l'apparition des unités " matière-énergie » qui en modifient certaines règles générales. Ce sont: 
$1^{\circ}$ La période de température-pression ascendante et descendante jusqu'aux environs de $100^{\circ}$ de température et du degré de pression atmosphérique à la surface de la terre, pendant laquelle se sont formés les électrons, les atomes, les molécules simples et la plupart de leurs composés, notamment l'eau à l'état liquide.

$2^{\circ}$ La période de l'eau, qui par ses propriétés de dissolvant et de catalyseur général, ainsi que par la réalisation des conditions de température et de pression à peu près uniformes pour presque toutes les réactions, rappelle à la vie un grand nombre de composés qui, sans l'intervention de l'eau resteraient inertes, parce que trop stables.

Il s'établit ainsi, dans ce nouveau milieu, des lois de structure et de sélection nouvelles, qui conduiront à la formation des colloïdes organiques et par là, à la formation des organismes.

$3^{\circ} \mathrm{La}$ période de la substance cérébrale et de son énergie la pensée.

Relativement à la durée des deux périodes précédentes que l'on peut évaluer à des millions de millions de siècles, on peut dire que cette dernière période ne fait que commencer, elle peut être évaluée à quelques centaines, tout au plus à quelques milliers de siècles, si l'on compte, bien entendu, son apparition non pas depuis la formation des premiers cerveaux rudimentaires, tels que nous les trouvons chez les animaux, mais à partir du moment de la formation d'un cerveau réellement pensant et conscient de ses actes.

L'apparition d'un tel cerveau a modifié de nouveau les conditions générales de l'évolution parce que, en faisant agir les formes d'énergie qui lui sont propres: 
la conscience et l'intelligence, il rappelle à la vie les unités devenues inertes et devient un nouveau facteur de sélection.

Le cerveau de l'homme ne sera peut-être jamais capable de modifier une loi naturelle quelconque, mais il est déjà devenu conscient du mécanisme et des lois d'un grand nombre de formes d'énergie qui se manifestent dans son milieu extérieur et dans son milieu intérieur, et est même arrivé à créer des formes d'énergie nouvelles. Il a reconnu la nécessité de bien connaître la structure et les lois de l'évolution de toutes les unités matière-énergie qui composent son milieu extérieur, pour bien comprendre la structure et le fonctionnement de son milieu intérieur, de l'organisme aux dépens duquel il se développe et pour rendre ainsi sa propre évolution plus sûre et plus rapide.

Avec la formation du cerveau pensant commence donc une période d'évolution nouvelle, parce que l'intervention de la pensée, la forme d'énergie consciente de ses buts, modifiera de nouveau les conditions de l'évolution des unités-énergie plus élevées.

On pourrait dire encore, que les trois grandes périodes de l'évolution sont caractérisées par la nature des énergies qui entrent en jeu : la première, par les énergies purement physiques; la seconde, par les énergies physiques et chimiques; la troisième, par les deux premières et par les énergies psychiques; mais il ne faut pas perdre de vue que toute énergie, quelle qu'en soit la nature, ne peut être qu'un mouvement, ou plus exactement, la résultante des mouvements coordonnés d'un nombre plus ou moins grand d'unités matérielles réunies en en- 
sembles d'équilibres dynamiques, que, par conséquent, toute énergie est, en dernière analyse, de nature physique.

\section{1. - Le rôle de l'organisation des unités matière- énergie en associations de plus en plus complexes dans l'accroissement progressif de la puissance d'action de l'énergie.}

Nous ne pouvons pas faire autrement que de nous représenter l'univers comme un espace infini contenant dans ses différentes régions de la matière et de l'énergie en quantités infinies et variables. Il ne peut donc pas être question de diminution ou d'accroissement de matière ou d'énergie daris l'univer's pris dans son ensemble. Mais, si l'univers représente ainsi un ensemble d'équilibre constant, il est composé en réalité d'une quantité infinie de régions distinctes qui se trouvent, à chaque moment, à des degrés d'évolution différents, qui ne sont donc pas à l'état d'équilibre constant les uns par rapport aux autres, qui évoluent et qui, du fait de cette évolution, subissent constamment des pertes de matière et d'énergie au profit des régions environnantes. Toutefois, au point de vue du travail qu'elle aura à exécuter, une unité "matière-énergie " n'a de valeur qu'autant qu'elle est organisée. Les unités matièreénergie éparses, sans liens déterminés, c'est-à-dire non organisées, ne sauraient exécuter aucun travail plus élevé sans s'organiser, c'est-à-dire se réunir entre elles en unités distinctes possédant un milieu intérieur pourvu d'un équilibre dynamique durable, et c'est là précisément en quoi consiste l'évolution progressive : dans l'organisation des unités matière- 
énergie d'un ordre pour la construction d'unités matière-énergie de l'ordre immédiatement suivant et plus élevé.

Les unités d'un ordre ne possèdent qu'exclusivement la forme d'énergie nécessaire pour exécuter le travail de construction des unités de l'ordre suivant et elles perdent, en exécutant ce travail, une partie de leur énergie propre sous forme d'énergies rayonnantes, mais en utilisant l'énergie qui leur reste à l'organisation d'une unité matière-énergie nouvelle, plus complexe, elles créent une forme d'énergie nouvelle, dont la valeur "travail" ou la puissance, devient infiniment plus grande que celle de la somme des énergies rayonnantes perdues.

De plus, il est nécessaire de faire remarquer ici, que l'énergie nouvellement formée est une forme d'énergie du milieu intérieur de l'unité nouvellement formée et que ces énergies du milieu intérieur, ne sont pas rayonnantes, pas transmissibles et par conséquent ne peuvent plus être perdues.

L'affinité chimique, par exemple, est une de ces énergies du milieu intérieur de la molécule. Elle peut ne pas se manifester dans certaines conditions de milieu, mais elle ne peut être jamais perdue ni même diminuée ou augmentée, tant que la molécule elle-même ne sera détruite.

Ainsi, dans le cours de l'évolution, les quantités des énergies perdues par rayonnement dans l'espace sont compensées et au delà, paŕr les qualités, par la puissance des moyens de travail dont dispose chaque unité matière-énergie résultant de l'organisation des unités de plus en plus complexes à chaque étape de cette évolution.

On peut dire que, si, par l'évolution progressive, 
la quantité totale de l'énergie comprise dans un espace déterminé diminue, la puissance des moyens mis en œuvre augmente progressivement à chaque étape de synthèse.

\section{2. - Discontinuité et continuité de l'évolution. Rôle des catalyseurs. Eléments de structure et élé- ments de travail.}

Présentée de cette façon, l'évolution générale apparaît comme un phénomène discontinu. En réalité, les liaisons entre les échelons successifs de synthèse sont assurées par l'évolution individuelle des espèces d'un même ordre d'unités, déterminée par les conditions du milieu de chaque moment, ainsi que par les lois de structure et de sélections.

L'étude de ces évolutions individuelles, à partir de la cellule, par conséquent des lois de l'évolution biologique, fera l'objet des chapitres suivants, l'étude de l'évolution des unités des ordres inférieurs étant du domaine de la physique et de la chimie pure, nous n'avons pas à nous en occuper ici.

Mais avant de clore ce chapitre, il nous semble important de faire remarquer encore, que tout cataclysme dans le monde des molécules, comme toute révolution dans la société humaine, ne peut que marquer un ou plusieurs pas en arrière dans l'évolution ascendante, parce que ces cataclysmes ne peuvent que détruire les organisations existantes et retransformer en énergies rayonnantes qui se perdent dans l'espace, les énergies déjà organisées, e'est-àdire le travail utile déjà accompli. Elles bouleversent l'équilibre entre les lois de structure et de sélections établies par les conditions de milieu générales du 
moment et rappellent à l'activité créatrice des individus qui ne se seraient pas imposés spontanément.

Eviter ces cataclysmes, sera l'œuvre du perfectionnement progressif de l'unité matière-énergie, dont le règne commence : du cerveau-pensée, - c'est donc à ce perfectionnement le plus rapide et le mieux compris que devraient tendre tous nos efforts.

\section{3. - Règles générales de l'évolution des milieux intérieurs. Sélection par l'intelligence. Tempéra- ture constante.}

Nous avons vu qu'il y a une uniformité très grande dans le mécanisme général de la formation des unités matière-énergie de toutes les catégories. Il y a bien des différences dans les détails : la réunion des unités différentes d'un ordre en unités plus complexes par condensation et par combinaison dans le monde inorganique, fait place à la différenciation dans le monde vivant; mais les règles générales de toutes ces formations sont partout les mêmes.

Nous l'avons déjà dit, mais il n'y a aucun inconvénient à le répéter encore une fois ici, puisque le but principal de ce travail est de faire ressortir ces lois : la règle générale du mécanisme de la formation de toutes les unités matière-énergie de tous les ordres de plus en plus complexes, c'est l'association des éléments différents en ensembles organisés pour un travail coordonné commun.

Ceci admis, il est intéressant de se rendre compte encore : $1^{\circ}$ quel est le mécanisme de l'organisation et du fonctionnement intérieur de ces associations; $2^{\circ}$ si ce mécanisme est le même pour les unités de tous les ordres. 
II nous faut reconnaître qu'il est encore impossible de trouver dans les données connues une réponse également satisfaisante à chacune de ces deux questions.

Les moyens d'investigation dont nous disposons actuellement ne nous permettent d'examiner en connaissance de causes, que l'organisation et le fonctionnement intérieur, ou, en d'autres termes, le milieu intérieur, des unités matière-énergie les plus élevées : des animaux supérieurs et en particulier de l'homme.

Comme tout autre, l'organisme de l'homme est composé essentiellement de quatre sortes d'éléments:

a) Eléments de structure: tissus osseux et conjonctifs qui donnent à l'ensemble une forme déterminée ;

b) Eléments de nutrition : appareils digestif, respiratoire, musculaire, circulatoire, glandulaire et génital, qui assurent l'assimilation, la désassimilation et la reproduction.

c) Eléments de sensibilité inconsciente: système nerveux appelé sympathique, qui règle automatiquement la nutrition de l'ensemble.

d) Eléments de sensibilité consciente : le cerveau, qui règle d'une façon en apparence volontaire les relations de l'organisme avec son milieu extérieur, - qui a par conséquent la faculté de constater sa propre existence et d'apprendre, par l'observation et l'expérience, à connaître le fonctionnement et la structure du milieu intérieur de l'ensemble dont il fait partie, comme de toute autre chose du milieu extérieur, dans lequel il vit.

Les deux systèmes nerveux se complètent réciproquement. Le système sympathique fonctionne comme s'il "savait" ce qui se passe ou ce qui doit 
se passer normalement dans toutes les régions, dans toutes les cellules du milieu intérieur de l'organisme, comme s'il avait conseience de toute modification, de toute rupture d'équilibre intérieur, ainsi que des mesures à prendre pour rétablir cet équilibre, comme si, en un mot, il avait conscience de sa structure, de ses moyens d'action ainsi que des moindres détails du fonctionnement particulier et des relations réciproques de tous les appareils, de toutes les cellules de l'ensemble. Le système sympathique est-il réellement conscient de son existence? Il nous est impossible de l'affirmer ou de le nier, mais, à en juger par la perfection et la sûreté de ses réactions, il est plus que probable qu'il agit d'une façon purement automatique, sans avoir conscience de son existence et de ses actes. Il n'a pas conscience du milieu extérieur à l'organisme, mais comme tout changement du milieu extérieur, tout agent non habituel qui pénètre de l'extérieur dans le milieu intérieur, en trouble plus ou moins l'équilibre fonctionnel normal, le système sympathique en est averti aussitôt et il en avertit le cerveau qui, à son tour, ayant conscience du milieu extérieur, peut réagir contre ce trouble et cherche à rétablir l'équilibre rompu, suivant le degré de conscience, ou, en d'autres termes, de connaissance des causes et des effets des réactions entre le milieu intérieur et extérieur et entre les différentes fonctions des tissus, organes ou appareils du milieu intérieur de l'organisme.

Le système cérébral n'est donc mis en rapport avec le milieu intérieur de son organisme et ne peut réagir sur lui que par l'intermédiaire du système sympathique, et encore il ne peut le faire qu'indirectement, par des agents étrangers, par des substances (médi- 
caments) qui, directement ou par l'intermédiaire du système glandulaire, exercent une action stimulante ou calmante sur les centres sympathiques.

Il nous est, en effet, encore impossible de calmer une douleur ou de provoquer une sensation agréable, de stimuler ou de modérer une sécrétion glandulaire par un simple acte de volonté, et nous ne le pouvons pas pour cette bonne raison que, n'ayant pas conscience, ne connaissant à peu près rien du mécanisme intime des réactions de notre organisme, du "comment " et du "pourquoi » de ses réactions, nous ne savons vouloir que d'un façon trop vague, nous ne pouvons pas donner des ordres précis au centre sympathique ou à l'organe dont l'équilibre fonctionnel est troublé.

Au point de vue de l'évolution individuelle ainsi que de l'évolution progressive, les quatre tissus qui constituent l'organisme de l'homme ont donc une importance très inégale.

Il s'est fait dans le milieu intérieur de l'unité matière-énergie "homme " une différenciation à plusieurs degrés que nous constatons sans en connaître le mécanisme intime, mais nous pouvons affirmer, sans crainte de nous tromper, que cette différenciation est le résultat d'une sélection des micelles, dont les plus actives et les plus sensibles aux réactions sont devenues les éléments constituants des cellules cérébrales et que le tissu cérébral, une fois formé, domine complètement toutes les fonctions de tous les autres tissus et détermine ainsi le développement de l'ensemble et son propre développement aux dépens de tous les autres tissus.

Il les fait travailler et il vit de leur travail.

Il n'en vit pourtant pas en parasite, parce que dans 
cette association, une sorte de symbiose, de différents tissus et organes, c'est le tissu nerveux qui, de par sa composition d'éléments les plus mobiles, doit être nécessairement aussi le plus actif, de sorte que s'il s'impose à son milieu, c'est uniquement par la quantité et surtout par la qualité du travail qu'il fournit.

Il "ressent» la nécessité d'assurer les meilleures conditions d'existence et de développement des tissus et organes qui le font vivre et il se conforme à çette nécessité, parce qu'il "comprend" qu'en agissant ainsi, il assure les meilleures conditions de son propre développement.

Dans cette association, le tissu cérébral est done l'élément sélectionné par le milieu intérieur de l'organisme. Une fois formé, il dirige le développement de ce milieu et devient ainsi, par son activité consciente, la pensée, le facteur de la sélection des éléments de toutes sortes les plus appropriés, pour faire monter l'évolution progressive de la matière à un échelon plus élevé.

Est-il possible de retrouver une organisation et une structure du milieu intérieur analogues dans les unités d'ordres inférieurs, ou, en d'autres termes, est-il possible de trouver ou de supposer chez les végétaux et les protistes dépourvus d'un tissu nerveux différencié, dans le milieu intérieur, des micelles organiques et inorganiques, des molécules, des atomes, des électrons, un élément ou un système qui dominerait par son activité tous les autres éléments, dont ce milieu intérieur est composé et qui deviendrait par là l'élément créateur des associations ou des différenciations plus élevées?

On peut l'affirmer pour tous les êtres vivants. Entre l'homme et les animaux vertébrés pourvus 
d'un cerveau analogue à celui de l'homme, il n'y a que des différences de quantité et de qualité. Chez les animaux dépourvus de cerveau proprement dit, c'est au système nerveux sympathique qu'appartient le ròle d'élément déterminant et créateur. Chez les protistes et les végétaux dépourvus de système nerveux différencié, ce rôle peut être attribué aux centrosomes ou à certains éléments du noyau.

$\mathrm{Au}$ delà, c'est encore l'inconnu.

Pour savoir ce qui se passe dans le milieu intérieur d'une molécule, il faudrait en connaître non seulement la structure apparente ou ce que l'on pourrait appeler la morphologie de la molécule telle que nous le montre l'étude des corps radioactifs, mais encore les différences des propriétés chimiques des différents éléments qui la composent.

La complexité de composition et de structure d'une molécule simple, que l'on peut comparer à une association d'un nombre plus ou moins grand de systèmes solaires, justifie pleinement la supposition que tous les éléments qui la composent n'ont pas des propriétés identiques, les uns par rapport aux autres et dans leurs relations avec l'ensemble.

Nous avons vu que la forme d'énergie dominante de la molécule, l'énergie créatrice des synthèses plus élevées, est l'affinité chimique; il s'agirait donc de savoir si cette propriété résulte d'une collaboration égale de tous les éléments, ou si elle n'appartient qu'à un certain nombre de ces éléments, qui se distingueraient par une activité spécialisée et plus grande.

D'après Soddy (1), le pouvoir de combinaison chi-

(1) Fr, Soddy: Le Radium, p. 308. Félix Alcan, éditeur, Paris (édition 1920). 
mique d'un élément doit s'expliquer par la tendance de l'atome, soit d'attirer à lui, soit d'abandonner un ou plusieurs électrons; il s'agirait donc de savoir si la liaison entre deux molécules différentes est ainsi assurée par des électrons quelconques ou par des électrons choisis de par une activité spéciale.

Il nous faut encore laisser cette question sans réponse, mais il nous a semblé utile de la signaler dans l'intérêt de la recherche d'une loi générale s'appliquant à la structure et à l'évolution des unités de tous les ordres, comme nous avons cherché à en établir une pour expliquer l'enchaînement de l'évolution progressive.

Pour nous faire une idée aussi complète que possible des conditions, dans lesquelles l'évolution progressive s'est effectuée jusqu'à présent et pourra peut-être continuer à l'infini, il nous faut prendre en considération encore un facteur de cette évolution, et notamment la stabilisation de plus en plus grande des conditions de température et par là des réactions dans les milieux intérieurs, à mesure que ces milieux deviennent plus complexes.

Cette stabilisation assure, en effet, aux unités qui en sont pourvues (les animaux supérieurs), une continuité et une régularité des réactions, qui aug.mentent considérablement leur puissance de travail et les rendent, dans une large mesure, indépendantes des variations du milieu ambiant.

La genèse de ce phénomène peut être expliquée sans trop de difficulté.

On sait que les réactions chimiques peuvent être endo- ou exothermiques. Dans le premier cas, la combinaison entre deux éléments se fait avec absorp- 
tion, la décomposition avec dégagement de chaleur. Ges réactions sont relativement très rares, elles donnent naissance à des composés dont la stabilité augmente avec la température. L'acétylène en est un exemple. Il se décompose très facilement et même avec explosion quand il est comprimé, à la température ordinaire; le carbone se combine, au contraire, avec l'hydrogène à la température de $3.500^{\circ}$ et le composé $\mathrm{C}^{2} \mathrm{H}^{2}$ doit être, suivant Le Chatelier, très stable à la température du soleil, vers $7.000^{\circ}$.

Dans la plupart des cas, les réactions sont exothermiques: les combinaisons dégagent de la chaleur et la stabilité du composé diminue avec l'accroissement de la température.

Quel est le mécanisme du dégagement et surtout de l'absorption de la chaleur par les combinaisons ? C'est là un problème dont l'étude n'a pas encore pu être abordée. Il faut supposer que ces manifestations thermiques sont déterminées par des réactions intraatomiques, mais pour le moment nous sommes obligés de nous borner à constater que les combinaisons endothermiques ont pour effet de refroidir le milieu dans lequel elles s'effectuent, les exothermiques, de l'échauffer, et que les décompositions agissent en sens contraire, de sorte que, si on réalise un certain nombre de ces réactions dans les deux sens dans un milieu fermé, séparé du milieu ambiant par des parois plus ou moins isolantes, on obtiendra dans ce milieu isolé une température qui sera la résultante des différences entre les chaleurs dégagées et absorbées.

La constance ou la variabilité de cette température dépendra de la rapidité, de la continuité et de la régularité de la succession des réactions, ainsi 
que de la conductibilité calorique des parois du milieu intérieur.

Or, nous avons vu que, dans l'évolution progressive, ce sont toujours les éléments les plus mobiles et les plus actifs qui s'associent pour former les unités matière-énergie plus élevées. Il arrive donc, quand ces milieux deviennent suffisamment complexes et volumineux, que les réactions deviennent d'une continuité, d'une régularité et d'une rapidité suffisantes pour assurer à l'ensemble une température à peu près constante, qui, une fois établie, devient à son tour un des facteurs les plus importants de l'évolution progressive, parce qu'il la rend indépendante, dans une certaine mesure, des variations du milieu extérieur.

On peut parfaitement supposer, en effet, que, si la température générale à la surface de la terre s'était abaissée au-dessous de zéro au moment où l'évolution progressive était au stade pré-nerveux (protistes et végétaux), l'évolution se serait arrêtée forcément à ce stade par suite de la congélation de l'eau, qui rend impossible toute réaction vitale chez des êtres dépourvus d'une température propre.

On peut parfaitement admettre aussi que, si cette éventualité se réalisait actuellement, à la période d'évolution du cerveau tel qu'il est aujourd'hui, elle n'aurait plus les mêmes inconvénients.

Le milieu intérieur "homme" possède une température assez constante pour la conserver malgré des variations considérables de la température ambiante et il est très probable que si, dans un avenir plus ou moins lointain, toute la surface de la terre devenait aussi froide que le sont actuellement les régions polaires, l'homme saura bien trouver les 
moyens d'assurer son existence, de vivre lui-mème et de faire vivre les animaux et les plantes qui lui seront nécessaires en mettant en valeur l'énergie intraatomique.

On ne sait pas encore comment cela se fera, mais on sait que les atomes contiennent des trésors d'énergie inépuisables (calorique et autres), et il n'est pas douteux qu'on arrivera à les exploiter et à produire les quantités de chaleur, de lumière et d'autres énergies rayonnantes nécessaires pour l'entretien et l'évolution progressive de la vie, bien avant que ne tariront leurs sources naturelles dans le soleil.

\section{4. - Conclusions du Livre Premier}

La conception de la constitution et de l'évolution de l'univers, accessible à notre conscience actuelle, peut donc s'appuyer sur l'analyse et la coordination des faits suivants:

L'univers est composé d'un certain nombre d'unités : matière, énergie distinctes, que l'on pouvait tout d'abord diviser en deux grandes classes:

$1^{\circ}$ Les unités qui semblaient indivisibles, dont la durée avait été considérée, jusqu'à ces derniers temps, comme illimitée, et dont la structure ainsi que la nature nous apparaissaient comme constantes, c'est-à-dire indépendantes des variations du milieu dans lequel elles pouvaient se trouver. C'étaient, pour les physiciens du siècle dernier, les atomes des corps simples: hydrogène, oxygène, azote, carbone, fer, etc....;

$2^{\circ}$ Les unités qui ont, comme individus, une existence éphémère parce qu'elles subissent, sous l'influence des variations du milieu extérieur, des trans-

Danysz. - La genèse de l'énergie psychique. 
formations incessantes, qui naissent et qui meurent.

La découverte des corps radioactifs, ainsi que les faits révélés par l'étude spectroscopique des astres, a fait reculer la limite des unités indivisibles et infiniment durables, et l'a reportée aux électrons, et on peut prévoir déjà une dissociation possible de ces derniers en éléments encore plus petits et plus simples : les éthérons.

L'étude du mécanisme de la dissociation spontanée de l'atome de l'uranium et d'autres corps radioactifs nous a permis de nous faire une idée assez précise de la structure intime et de la composition de l'atome. Nous ne connaissons encore rien de la structure et de la composition des électrons, mais comme nous savons que les atomes sont composés d'électrons, nous pouvons admettre, que la structure de ces derniers est plus simple, et nous sommes autorisés à supposer que leur durée est plus longue. Nous savons aussi que l'énergie électronique : l'électricité, peut se manifester dans des conditions de milieu beaucoup plus variables que l'énergie earactéristique des atomes: l'affinité, et par conséquent, que les électrons doivent être d'une constitution plus stable que les atomes.

Peut-on considérer aujourd'hui l'électron comme l'unité matière-énergie primordiale, infiniment durable, ou bien faut-il chercher cette unité encore plus loin, dans l'éthéron? Peu importe! Théoriquement, pour le bon sens, la divisibilité d'une unité matérielle quelconque est infinie; mais s'il y a des éthérons, ils sont nécessairement bien plus simples, stables et durables que les électrons et on peut admettre que la durée de leur existence individuelle dépasserait alors celle des systèmes solaires de notre uni- 
vers, et cette certitude peut nous suffire, pour placer l'électron ou l'éthéron à la base de l'évolution progressive comme unité de matière-énergie primordiale, uniforme et infiniment stable et durable.

Dans la classification que je viens de proposer, on devrait donc placer: d'un côté les éthérons ou les électrons, de l'autre côté toutes les autres unités connues. L'on constate alors, que toutes ces unités que nous connaissons aujourd'hui n'existaient pas toujours, et que si on peut admettre, à la rigueur, l'existence simultanée d'électrons, d'atomes et de sels dans un milieu quelconque, la formation des composés organiques n'est devenue possible qu'après la condensation de l'eau à la surface de la terre, c'est-àdire dans un milieu de température inférieure à $100^{\circ}$. Nous constatons en outre, que depuis cette époque, la formation des unités de plus en plus complexes, n'a pas été simultanée, mais que ces unités: les êtres unicellulaires et pluricellulaires de plus en plus différenciés, ont apparu successivement dans un ordre déterminé.

Mais si nous n'en avons pas la certiture scientifique, nous avons, depuis la découverte du fait de la décomposition des atomes en unités plus simples, des raisons sérieuses d'admettre une évolution analogue pour les unités inorganiques.

Cette succession nécessaire dans la formation des unités "matière-énergie " d'une puissance énergétique de plus en plus grande, par la réunion et la coordination, pour un travail commun, des unités plus simples en unités plus complexes dans le monde inorganique, par la multiplication et la différenciation des cellules dans le monde vivant, une fois admise comme l'hypothèse de la genèse aujourd'hui 
la plus probable, nous avons cherché à en faire ressortir les facteurs et nous avons vu:

$1^{\circ}$ Que, dans la période inorganique, le facteur des condensations et des combinaisons ne pouvait être autre que l'abaissement graduel et continu de la température qui a eu, en même temps, pour conséquence nécessaire, une sélection des éléments moins stables et plus actifs qui, seuls, pouvaient ainsi devenir les composants des unités plus complexes;

$2^{\circ}$ Que la formation de l'eau à l'état liquide, à la surface de la terre, et l'abaissement progressif de la température de cette eau jusqu'à $50^{\circ}$ environ a été le facteur d'une deuxième sélection, comme dissolvant de différents éléments et composés, en proportions différentes, et, comme catalyseur d'une synthèse des éléments dissous et dissociés, en proportions telles, qu'ils étaient précisément nécessaires pour former la matière vivante.

La formation des premières unités vivantes n'exigeait donc pas l'intervention d'une énergie spéciale. Elle était la conséquence naturelle et même obligatoire d'une série de sélections qui ont abouti à la réunion dans l'eau, à un moment donné, des éléments les plus légers, les plus riches en affinités multiples, capables de former les composés les moins stables, le plus facilement modifiables qui, en un mot, par le seul jeu de leurs affinités, aidées par les propriétés catalysantes de l'eau, ont dû donner naissance aux premières micelles vivantes.

Il ne s'en forme pas actuellement?

Il ne se forme pas spontanément des microbes dans un bouillon de culture dans lequel certains microbes ensemencés se multiplient bien. C'est une certitude indiscutable depuis les mémorables expé- 
riences de Pasteur; mais il faut peut-être des siècles de formations successives et des conditions de milieu que personne n'a encore cherché à réaliser, pour qu'une micelle primitive devienne microbe.

$3^{\circ}$ Nous avons vu ensuite, qu'une fois les êtres vivants constitués, comme unités pourvues d'un milieu intérieur d'une complexité infiniment plus grande que celle de toutes les unités qui les ont précédées, et pourvues, grâce à cette complexité, d'une sensibilité très grande à toutes les actions du milieu extérieur, ce sont les énergies rayonnantes qui sont devenues les facteurs les plus actifs de l'évolution progressive, en provoquant la formation des organes des sens et par là du tissu nerveux.

$4^{\circ}$ Que ce tissu nerveux et ses différentes formes d'énergie ont conduit cette révolution jusqu'à l'homme, c'est-à-dire à un cerveau, dans lequel les sphères d'association ont acquis un développement dominant toutes les autres parties de l'encéphale, et à son énergie spécifique : l'énergie psychique, qui résulte de la coopération coordonnée de toutes les unités "matière-énergie " précédentes et qui est devenue d'une puissance telle, qu'elle ne subit plus passivement les réactions du milieu extérieur, mais qui, devenue consciente d'elle-même, de sa force et de ses moyens d'action, cherche à intervenir activement dans l'évolution de son milieu intérieur et à y adapter le milieu extérieur.

J'ai cherché à expliquer encore pourquoi cette évolution est progressive, pourquoi elle ne s'est pas arrêtée en route et doit être sans limite, en montrant qu'à chaque degré de complexité la puissance énergétique des unités nouvellement formées, augmentait dans des proportions toujours croissantes, et que 
cet accroissement de puissance était une conséquence nécessaire de la succession des associations coordonnées de toutes les unités " matière-énergie " précédentes, qui ne s'additionnent pas simplement les unes aux autres, mais s'organisent pour une action commune.

Et enfin, j'ai cherché à démontrer qu'à partir de l'apparition des mammifères, un des facteurs les plus importants de la continuité et de la rapidité de l'évolution, était la constitution d'un milieu intérieur à température de plus en plus constante, condition indispensable d'une continuité régulière des réactions du milieu intérieur et d'une grande résistance aux influences perturbatrices des variations de température du milieu extérieur. 


\section{LIVRE II}

\section{RÉACTIONS DE LA MATIÈRE VIVANTE}

\section{5. - Evolution naturelle spontanée}

Ln être vivant, végétal ou animal, naît, s'accroît, meurt et subit dans cette évolution certaines règles ou lois naturelles générales. Et quand les conditions de développement d'un être vivant ne dépendent que du milieu naturel dans lequel il vit, quand aucune action volontaire et consciente de son but ne vient en modifier le cours, on peut dire qu'il subit une évolution naturelle spontanée.

Telles devaient être les conditions de l'évolution des espèces animales et végétales à la surface de la terre pendant les millions de siècles avant l'apparition de l'homme savant, conscient de ses actes.

Les premières observations riches en conséquences qu'a dû faire l'homme, obligé de vivre aux dépens de la flore et de la faune spontanées qui l'entouraient, ce sont les différences très grandes dans le développement des mêmes espèces sur les sols de nature différente. Il constatait que les terrains secs et sablonneux n'étaient recouverts que d'une végétation maigre en quantité et en qualité, tandis que les mêmes espèces poussaient en abondance sur des terres noires et humides; quand il a pu voyager au 
loin il a reconnu, en outre, la variété infinie des plantes et des animaux qui se sont développés sous l'influence des climats différents.

C'est ainsi que l'on a appris, tout d'abord, que ce sont les conditions de milieu dans lequel on vit, et surtout la nature du sol et la température, qui déterminent les conditions de développement des végétaux et, par là, des animaux qui s'en nourrissent. Ce sont des considérations basées sur ces observations très simples qui ont permis à Lamarck de formuler sa théorie de l'évolution des espèces. C'est le milleu qui crée l'espèce, et c'est l'obligation de s'adapter à d'autres milieux qui a déterminé la différenciation des races et la formation d'espèces nouvelles, chaque fois que les modifications apparues dans l'organisme de l'individu sont devenues transmissibles par hérédité.

Darwin a introduit dans la théorie de l'évolution des espèces un autre facteur important: la sélection naturelle qui, dans la lutte pour la vie, favorise le développement des individus les mieux armés; mais, n'est-il pas évident que seuls les individus dont lorganisme fut préalablement modifié par les conditions du milieu pouvaient mettre à profit les qualités acquises et les transmettre à leurs descendants?

On ne peut donc chercher la cause première de la formation d'un être vivant déterminé, de sa structure et de ses propriétés, que dans la composition du milieu et dans le climat dans lequel il s'est formé; les modifications de structure qui différenciaient les espèces, n'ont pu être déterminées que par des changements du milieu, dans lequel elles ont vécu ensuite, et ce n'est qu'après l'accomplissement de ces modifications, que la sélection naturelle a pu inter- 
venir pour donner la victoire aux individus et aux espèces les mieux armés pour la lutte.

Or, s'il en est ainsi, comment peut-on comprendre les relations entre le milieu et la formation ainsi que la différenciation des êtres vivants?

Les théories de Lamarck et de Darwin, ainsi que celles de leurs continuateurs, les néo-lamarckiens et les néo-darwinistes: Weissmann, W. Roux, Haeckel, etc... répondent à cette question par des arguments tirés exclusivement des observations biologiques; de la paléontologie, qui montre une succession régulière dans l'apparition à la surface de la terre de plantes et d'animaux d'une organisation de plus en plus élevée; de l'embryologie, qui nous apprend qu'aux premiers stades de leur évolution, tous les êtres vivants se ressemblent; de l'anatomie, de l'histologie, de la physiologie comparée, qui montrent des passages insensibles entre les formes de tous les organes et tissus des êtres différents et une uniformité très grande dans leurs fonctions.

Quant au mécanisme intime de l'action du milieu extérieur sur les organismes, nous en savons encore bien peu de choses; on peut même dire que, non seulement nous ne savons pas comment les différents facteurs du milieu extérieur que nous connaissons, ont pu déterminer la formation de races, de variétés et d'espèces nouvelles, mais nous sommes encore à nous demander souvent comment il faudrait faire, pour le chercher.

En effet, la période de simple observation des naturalistes de la première moitié du $\mathrm{xIx}^{\circ}$ siècle a fait place à une période d'expérimentation, à des recherches, par lesquelles on a voulu démontrer 
qu'en faisant varier le milieu, on peut faire apparaître des individus qui diffèrent, par certains caractères, de leurs parents; mais dans les expériences faites jusqu'à présent, les caractères nouveaux conférés à l'individu par une intervention artificielle ne sont pas transmis à sa descendance.

Deux ouvrages excellents qui viennent de paraitre dans la collection de Gustave Le Bon (Flammarion, Paris), La Chimie et la Vie, de G. Bohn et Drzewina, et L'hérédité et le milieu, de D.-P. Conklin, professeur à l'Université de Princeton, contiennent une foule de ces expériences et il n'est pas inutile d'en citer quelques-unes ici :

Ainsi, Herbst (1897) a montré, en élevant des œufs d'oursins dans l'eau de mer, dont il faisait varier la contenance en différents sels, que le potassium et le magnésium sont indispensables à la fécondation, le chlore et le sodium à la segmentation, que le calcium joue le rôle d'un ciment qui maintient ensemble les premières cellules qui se multiplient par des divisions successives. Dans un milieu dépourvu de soufre et de magnésium, le tuge digestif n'arrive pas à se former. Le potassium est indispensable à la formation des cils qui recouvrent tout le corps de la larve.

Stockard (1900) a montré que les larves du fundulus hétéroclitus, un poisson marin, acquièrent une particularité très curieuse, quand on les fait éclore dans une eau, à laquelle on a ajouté un excès de chlorure de magnésium: au lieu de deux yeux normaux, un grand nombre de petits poissons ne forment qu'un seul œil au milieu de la tête.

D'après les expériences de Clarke (1901), confirmées par Shinji (1918), le sulfate de magnésium, 
ainsi que les sels de certains autres métaux lourds (nickel, zinc, étain, mercure, plomb), les sels d'antimoine et même une certaine dose de sucre, dont on arrose les rosiers, font pousser des ailes à des pucerons (alphis rosæ) qui vivent sur ces rosiers et qui normalement n'ont pas d'ailes.

Dewitz a obtenu deś insectes aptères en soumettant leurs larves à l'action de l'acide cyanhydrique; Bohn a fait pousser douze bras à une méduse (Eleutheria dicholoma) qui, normalement, n'en porte que six, en la privant pendant quelques heures d'oxygène.

P. Marchal, ayant constaté que les acacias et les pêchers sont habités généralement par deux espèces différentes de cochenilles : l'acacia par le lecanium robiniarum; le pêcher par le $L$, corni, a transporté ce dernier sur l'acacia et a constaté qu'à la génération suivante, l'espèce du pêcher a pris tous les caractères du L . robiniarum.

Plus intéressants et plus importants sont les résultats des expériences, qui ont eu pour objet l'étude de l'action des substances contenues dans certaines glandes, sur le développement des animaux.

Ainsi, Gudernach (1912) a obtenu des grenouilles naines, pas plus grandes que des mouches, en nourrissant les têtards avec de la glande thyroïde de divers mammifères. Les mêmes têtards devenaient au contraire énormes et ne se métamorphosaient pas, quand on les nourrissait avee du thymus.

E. et M. Hoskins (1919) ont alors eu l'idée d'enlever l'ébauche de la thyroïde à des jeunes têtards de $6^{\mathrm{mm}}$ et ont constaté que si les têtards opérés s'accroissaient plus rapidement que les témoins, ils restaient à un état de développement inférieur. Les têtards opérés devenaient trois fois plus grands que les 
témoins au moment de la métamorphose, mais ne se transformaient pas en grenouilles. Chez ces têtards vivants, on constatait une insuffisance de la calcification. L'exagération de la croissance coïncidait avec une hyperplasie marquée de l'hypophyse. Le volume du cerveau, ainsi que celui du foie, dépassait sensiblement la normale, mais ni l'un ni l'autre n'étaient différenciés. Les branchies internes persistaient, mais les poumons s'étaient développés et fonctionnaient aussi. L'intestin, très allongé, a gardé son caractère larvaire. Les ovaires sont devenus volumineux et contiennent des ovocytes, mais ces derniers ne sont pas arrivés à maturation. Il n'y a ni œufs ni oviductes. Par contre, les organes mâles, parvenus à maturité, contiennent des spermatoroïdes.

Il est donc indiscutable que l'introduction dans l'organisme d'une substance étrangère, ou d'une substance habituelle en porportion anormale, peut modifier profondément la structure et les fonctions de l'individu, mais les faits que nous venons de citer font conclure à Conklin: "Les caractères développés, "acquis" ou non, ne sont jamais transmis par l'hérédité, et leurs changements n'affectent pas la constitution héréditaire du germe. "

Mais alors, peut-on se demander, s'il en est ainsi, si l'hérédité résiste toujours victorieusement aux changements éprouvés par l'individu, comment peuton admettre et expliquer l'évolution progressive, la succession d'êtres d'une organisation de plus en plus complexe et élevée?

On trouvera facilement une réponse satisfaisante à cette question en modifiant un peu la conclusion de Conklin, en disant: "Les caractères nouveaux qui apparaissent spontanément chez certains indi- 
vidus, ou dont l'apparition a été provoquée par l'expérience, ne peuvent être transmis par l'hérédité que s'ils provoquent des changements correspondants dans les cellules sexuelles. "(Nous y reviendrons plus loin.)

On ne peut pas affirmer qu'un caractère n'est pas héréditaire, parce qu'il n'apparaît pas chez les enfants exactement tel qu'il s'est manifesté chez l'un des parents. Il devient même de plus en plus certain aujourd'hui, qu'une telle exigence ne serait rien moins que raisonnable, parce que l'expérience nous a prouvé précisément, que dans un.organisme aussi complexe que celui d'un animal, une réaction peut avoir des répercussions aussi nombreuses que variées, que nous ne pouvons en apprécier que les apparences et ne connaissons rien, absolument rien de leur mécanisme intime.

A mesure qu'on cherche à l'approfondir, le problème du "comment" de l'évolution progressive devient de plus en plus complexe et tout ce que nous en savons aujourd'hui, c'est que, pour le résoudre, il faudra suivre les modifications provoquées ou constatées chez l'individu pendant une longue série de ses descendants, et avant tout, baser les observations sur une connaissance de plus en plus exacte des règles générales auxquellès la matière vivante et les organismes, de plus en plus complexes, doivent obéir dans leurs réactions.

\section{6. - Micelle albuminoïde vivante. Unité physiologique}

On sait que l'organisme d'un animal différencié, par exemple d'un mammifère, est composé de diffé- 
rents tissus qui, à leur tour, sont composés de cellules. Quand on examine les cellules au microscope, on les trouve composées d'un noyau plus ou moins volumineux, d'un plasma plus ou moins transparent ou granuleux et d'une couche périphérique plus dense : la membrane.

Examiné à l'ultra-microscope, le plasma cellulaire est composé d'un grand nombre de corpuscules briltants, animés d'un mouvement de va-et-vient incessant et baignant dans un liquide limpide.

La membrane, le noyau ainsi que les granules plus petites que l'on peut trouver dans une cellule, sont composés de corpuscules analogues quant à leurs formes et leurs dimensions apparentes, mais plus condensés. Ils sont baignés par le même liquide transparent.

On appelle ces corpuscules des micelles albuminoïdes.

Cette division en unités plus petites peut-elle être poussée encore plus loin?

Oui, si on considère un organisme comme un composé purement physico-chimique, et, dans ce cas, on pourrait dire encore, que la micelle est formée de molécules plus ou moins complexes, ces dernières de molécules simples (élémentaires) et ces dernières encore d'atomes.

Non, si on considère l'organisme comme une unité physiologique, c'est-à-dire un ensemble que l'on appelle individu vivant. Dans ce cas, pour conserver les propriétés essentielles qui caractérisent la vie, et que l'on ne trouve pas dans la matière inerte, la divisibilité s'arrête à la micelle albuminoïde. En décomposant les micelles, on fera mourir la matière vivante et l'on n'obtiendra que des substances minérales. 
La micelle albuminoïde est donc la plus petite unité physico-chimique et en même temps physiologique de la matière vivante, au même titre qu'une molécule composée est la plus petite unité de structure d'une substance minérale de composition définie (chlorure de sodium, sulfate de magnésie, etc.), un atome, la plus petite unité caractéristique d'un élément, tel que l'oxygène, l'azote, le fer, etc.

En considérant un organisme différencié au point de vue de sa fonction essentielle, c'est-à-dire de sa nutrition, on constate qu'un tel organisme ne peut vivre qu'en nourrissant ses cellules, et qu'il ne peut remplir cette fonction qu'en apportant au niveau des cellules un liquide composé d'eau contenant des sels inorganiques et organiques en solution. Ce liquide baigne toutes les cellules, et ce sont ces composés inertes cristalloïdes seuls qui pénètrent normalement à l'intérieur des cellules et deviennent ainsi les matériaux de construction des micelles.

Bien entendu, un animal peut absorber des substances albuminoïdes vivantes ou mortes, végétales ou animales, et s'en alimenter, mais ces substances ne peuvent pénétrer normalement dans le sang et devenir nutritives pour les cellules, qu'après avoir été digérées, c'est-à-dire dégradées en composés simples, solubles dans l'eau. Cette transformation est indispensable parce que, au point de vue de leur structure et de leurs propriétés physico-chimiques, les substances albuminoïdes mortes sont très analogues aux mêmes substances vivantes. Ce sont des colloïdes formés de micelles, insolubles dans l'eau, qui ne peuvent pas traverser les membranes cellulaires normales. Dans le eas où cela deviendrait possible, une micelle vivante ne pourrait s'assimiler

\section{http://rcin.org.pl}


d'autres micelles mortes, qu'à la condition de les faire décomposer au préalable en cristalloïdes simples, isolés et solubles dans l'eau, exactement comme il ne serait possible de construire une maison avec d'autres maisons, qu'à la condition de décomposer ces dernières en matériaux de construction simples et plus ou moins isolés.

Ainsi, au point de vue du mécanisme le plus simple de la nutrition (y compris la respiration) qui caractérise la vie, une cellule quelconque faisant partie de l'organisme le plus différencié, par exemple de l'homme, se trouve exactement dans les mêmes conditions que l'organisme unicellulaire le plus simple, vivant dans l'eau.

On peut affirmer que toute cellule, quelle que soit sa complexité et le genre d'existence (aérien ou aquatique) de l'être dont elle fait partie, mène une vie aquatique.

L'analogie ne va pourtant pas plus loin. Entre un être uni- et pluricellulaire, il y a des différences de même ordre qu'entre une molécule et une micelle. L'être vivant, le plus parfait, l'homme, est une unité de matière au même titre qu'un électron, une molécule, une micelle ou une cellule. Ainsi que nous l'avons vu dans les chapitres précédents, toutes ces unités ne représentent en réalité que des degrés successifs de l'évolution de la même matière et, au point de vue physico-chimique, ne diffèrent entre elles que par la complexité de leur composition, mais il est important de noter, que pour chacun de ces degrés d'évolution, il se forme un équilibre d'ensemble, une communion et une collaboration intimes de toutes les unités d'ordres inférieurs réunis dans l'unité de l'ordre supérieur. 
Une cellule qui fait partie d'un organisme supérieur, n'est plus aussi libre et indépendante qu'un être unicellulaire vivant dans l'eau. Les réactions qu'elle aura subies se réfléchiront sur l'ensemble, dont elle fait partie et elle ressentira les réactions d'une portion quelconque de cet ensemble.

Il en est de même pour les micelles qui composent une cellule.

Nous ne possédons encore aucun moyen d'étudier la forme, la structure, le poids ni les dimensions des micelles albuminoïdes vivantes, mais à en juger par leur ressemblance avec les micelles des substances colloïdales simples (or, argent, platine, amidon, etc.), ainsi que par la régularité de leurs mouvements (mouvement Brownien), on peut admettre qu'elles sont sphériques et d'un diamètre compris entre 0,1 et $0,01 \mu$ (la lettre $\mu$, abréviation de micron, désigne le millième du millimètre), et comme le diamètre des molécules est évalué à 0,0001 - $0,0005 \mu$, on peut admettre aussi qu'elles sont composées de milliers ou de dizaines de milliers de molécules.

Les micelles des colloïdes simples, telles que, par exemple, celles des métaux ou de l'amidon, sont composées de molécules toutes identiques, dont on peut établir les formules exactes. Ainsi la formule d'une molécule d'oxychlorohydrate ferrique est $\left(\mathrm{FeO}^{3} \mathrm{H}^{3}\right) \mathrm{n}\left(\mathrm{FeCI}^{3}\right)$, celle d'une molécule d'amidon $\left(\mathrm{C}^{6} \mathrm{H}^{10} \mathrm{O}^{5}\right) \mathrm{n}$, mais il doit être bien entendu que, si les molécules de ces substances étaient indépendantes les unes des autres, ce ne serait ni de l'hydrate de fer colloïdal, ni de l'amidon, parce que les propriétés

DAnysz. - La genèse de l'énergie psychique. 
qui caractérisent l'état colloïdal sont déterminées précisément par la combinaison d'un grand nombre de ces molécules identiques en unités distinctes (1).

La composition moléculaire d'une micelle albuminoïde est très certainement toute autre. Elle est composée d'un grand nombre de molécules qui diffèrent les unes des autres par leurs structures et leurs propriétés. Ainsi quand on traite l'amidon par l'acide sulfurique ou chlorhydrique, on transforme les micelles de l'amidon en molécules de glycose et tous les amidons, quelle qu'en soit la provenance (riz, blé, pomme de terre), donneront toujours le même glycose, tandis qu'une substance albuminoïde, traitée de la même façon, sera décomposée en un certain nombre d'acides-aminés qui, tout en possédant tous le même groupement caractéristique $\left(\mathrm{CHNH}^{2}-\mathrm{COOH}\right)$ et, à peu près, la même composition élémentaire, seront très différents les uns des autres par leur structure et leurs propriétés.

On doit en conclure qu'une micelle albuminoïde est composée d'un certain nombre de molécules différentes.

En analysant de cette façon différentes substances albuminoïdes du sang, du lait, des muscles, de la substance nerveuse, etc., on constate aussi que ces albumines diffèrent les unes des autres par les proportions et la nature des acides-aminés dont elles sont composées.

Nous connaissons la composition élémentaire de l'albumine. En analysant toutes sortes de substances

(1) La formation d'un cristal résulte aussi de la réunion d'un grand nombre de molécules identiques, mais dans ce cas il n'y a qu'un simple accolement des molécules les unes aux autres, il n'y a pas de combinaison. 
albuminoïdes animales ou végétales, on a reconnu que toutes sont formées principalement de carbone, d'hydrogène, d'oxygène et d'azote et d'une vingtaine (1) d'autres métaux et métalloïdes en quantités relativement beaucoup plus faibles et en proportions variables. La composition élémentaire de toutes ces substances est done bien uniforme, mais comme on a constaté, d'autre part, que les albumines des différentes espèces possèdent des propriétés physico-chimiques et physiologiques différentes, il faut bien en conclure, que les micelles de toutes ces albumines, tout en étant formées des mêmes molécules, ne les contiennent pas en proportions identiques.

On a constaté, en outre, que les propriétés des substances albuminoïdes d'une espèce végétale ou animale sont très constantes : l'albumine du sérum d'un cheval normal présentera les mêmes propriétés que celle du sérum de tous les chevaux normaux.

On doit en conclure que les micelles albuminoïdes vivantes d'une espèce déterminée ne peuvent posséder un ensemble de propriétés physiologiques constantes qu'à la condition d'avoir une composition chimique et une structure également invariables. Or, une telle micelle absorbe sans cesse certaines substances et en élimine certaines autres. Les éléments qui entrent dans la micelle sont les mêmes que ceux qui en sortent, mais, à la sortie, on ne les trouve plus sous la forme des mêmes composés sous laquelle ils sont entrés. Les composés absorbés par la micelle ont été transformés en route, ils ont subi une évolution, et c'est la constance de la succession des phases de cette évolution qui détermine la cons-

(1) On a trouvé jusqu'à présent 32 éléments dans les différentes substances vivantes. 
tance des propriétés d'une micelle albuminoïde vivante. Une micelle albuminoïde ne peut donc pas avoir la même composition dans toutes ses parties.

Considérée à un moment de sa vie, que nous pouvóns imaginer arrêtée pour un instant, une micelle albumịnoïde serait formée d'une série de composés de plus en plus complexes résultant de synthèses successives et, à partir du composé le plus complexe, d'une autre série de composés de plus en plus simples résultant de décompositions successives.

La formation d'une telle micelle par la condensation et la combinaison d'un certain nombre de molécules identiques, est donc non seulement peu probable, mais tout à fait impossible.

$\mathrm{Au}$ point de vue physico-chimique, une micelle vivante ne peut être représentée que comme une unité de composition et de structure en équilibre dynamique constant, déterminé par une série de synthèses et de décompositions qui se succèdent d'une façon continue, toujours dans le même ordre, et s'effectuent à la même place.

$\mathrm{Au}$ point de vue de ses fonctions, une telle micelle doit être considérée comme une unité de travail qui, dans les cas les plus simples, chez les végétaux, puise dans son milieu ambiant les éléments nécessaires à sa nutrition à l'état de composés inorganiques (azotates, carbonates, phosphates, chlorures de sodium, calcium, potassium, etc.), en conserve une partie pour sa nutrition et sa multiplication et en élimine le reste sous forme de composés organiques (sels ammoniacaux, acide carbonique, hydrates de carbone, etc.).

Les micelles qui composent les cellules des animaux, ne peuvent plus se nourrir de matières exclu- 
sivement minérales; en plus des sels, il leur faut des composés plus complexes, des acides-aminés, qui résultent de la digestion des albumines végétales ou animales.

Les cellules végétales sont donc capables de faire la synthèse totale de leurs albumines, ou, en d'autres termes, de construire leurs micelles albuminoïdes avec les matériaux inorganiques les plus simples: azotates, phosphates, etc., dissous dans l'eau, l'oxygène, l'acide carbonique et quelquefois même l'azote de l'air. Les cellules animales ainsi que celles de beaucoup de végétaux parasites, ont perdu cette faculté, elles ne sont-capables de faire la synthèse de leurs albumines qu'à partir de composés organiques déjà assez complexes : les acides-aminés.

Ainsi, la différence entre le processus de nutrition d'une cellule végétale et d'une cellule animale consiste principalement dans ce fait, que la première peut opérer la synthèse totale des acides aminés et en construire ensuite les micelles albuminoïdes, tandis que la cellule animale en est incapable. Pour construire des albumines, elle est obligée de se procurer des acides aminés tout faits.

On doit en conclure, que les micelles qui forment la substance vivante des animaux, et surtout des animaux supérieurs, sont d'une composition et d'une structure physico-chimique plus complexe, plus élevée que celles des végétaux. Mais, quelles que soient les différences de complexité des micelles de différentes espèces, les réactions de nutrition qu'elles auront à subir seront partout de la même nature, sinon identiques dans leurs détails. Elles devront être toujours identiques dans tous leurs détails dans une micelle d'une espèce déterminée, pour que cette 
espèce puisse toujours ressembler à elle-même, c'est-à-dire conserver tous ses caractères spécifiques. Or, il est évident que les réactions ainsi que l'équilibre physico-chimique qui en résulte, sont strictement déterminés en premier lieu par la composition chimique élémentaire de la micelle et ensuite par les conditions de deńsité, c'est-à-dire de la quantité d'eau retenue dans l'ensemble, ainsi que de température, qui exerce une grande influence sur la durée de chaque réaction, de sorte que, pour remplir cet ensemble de conditions, une micelle vivante doit absorber toujours, dans les mêmes proportions, exactement les mêmes éléments, dont elle est formée et se maintenir dans un milieu, dont la nature physico-chimique (composition, densité, viscosité, etc.) ainsi que la température ne peuvent varier que dans des limites assez étroites.

Les conclusions que l'on doit en tirer s'imposent d'elles-mêmes: si l'état normal d'une micelle vivante dépend de sa composition élémentaire et reste étroitement lié aux conditions de composition, de densité et de température du milieu dans lesquelles elle vit, conditions dont l'ensemble détermine son équilibre physico-chimique et fonctionnel normal, il est évident que toute modification notable apportée à l'un quelconque de ces facteurs, aura pour résultat de déranger cet équilibre et de mettre la micelle dans un état anormal.

Ces troubles de l'équilibre normal peuvent être provoqués par des facteurs que l'on peut classer en plusieurs ordres suivant leur manière d'agir et notamment :

$1^{\circ}$ Les composés chimiques simples, les cristal- 
loïdes, qui ont pour caractère commun d'être solubles et dissociables dans l'eau; de traverser les membranes dialysantes, de pénétrer dans le milieu intérieur des cellules et des micelles albuminoïdes et de former des combinaisons directes et immédiates avec les substances qui composent les micelles.

$2^{\circ}$ Les composés chimiques complexes, les colloïdes qui ont pour caractère commun d'être insolubles dans l'eau, qui ne traversent pas les membranes dialysantes et ne peuvent pas former des combinaisons directes avec les substances qui composent les micelles albuminoïdes vivantes.

Les colloïdes qui pénètrent dans le milieu intérieur des cellules ou des organismes doivent être digérés, c'est-à-dire décomposés en cristalloïdes, pour être assimilés ou éliminés.

$3^{\circ}$ Les énergies rayonnantes: température, tumière, électricité, rayons $\mathrm{X}, \beta, \gamma$, etc., qui, suivant leur intensité et leur action élective sur certains éléments, peuvent modifier l'allure et la nature des réactions normales et provoquer des troubles plus ou moins graves.

$4^{\circ}$ Les émotions ou énergies d'ordre psychique, qui peuvent provoquer des réactions chimiques anormales en traublant les fonctions de l'organisme par l'intermédiaire des centres nerveux. Ces énergies ne peuvent agir, bien entendu, que sur des êtres pourvus d'un système nerveux différencié et d'un cerveau.

Nous n'avons aucun moyen de constater actuellement si, ou dans quelle mesure les micelles peuvent vivre en liberté, c'est-à-dire isolées du plasmạ de la cellule à laquelle elles appartiennent. Il ne nous est donc pas possible de contrôler les réactions 
intra-micellaires, pas plus que nous ne pouvons apprécier directement les réactions qui se passent entre les molécules de deux corps différents.

Mais, de même que nous ne pouvons connaître les propriétés des molécules invisibles qu'en opérant sur des quantités visibles et pondérables de la matière, il faudra nous borner à apprécier le mécanisme et les effets des réactions micellaires par déduction, d'après les réactions des cellules ou plutôt des tissus et des organes.

\section{7. - Réactions provoquées par les cristalloïdes}

Une série de recherches sur la fécondation artificielle inaugurée par J. Loeb, nous ont donné sur ce sujet les premiers renseignements précis.

J. Loeb, d'abord, et un grand nombre de naturalistes après lui (Delage, Bataillon, Lillie, Godlewski, etc.), ont montré qu'il est possible d'obtenir le développement des œufs d'oursin, d'étoile de mer, de certains vers, de poissons et même de grenouille (Bataillon), sans intervention des spermatozoïdes spécifiques. Dans tous ces cas, la fécondation naturelle par les spermatozoïdes avait été remplacée par une fécondation artificielle, au moyen de certains agents chimiques (sels, acides, alcalis, alcools, etc.) ou simplement physiques (agitation, piqûres), et le but de ces expériences était de démontrer que, pour obtenir d'un ovule la formation d'un être nouveau et complet, la réunion de deux éléments, mâle et femelle, n'était pas nécessaire, que l'élément femelle, l'ovule, était seul indispensable, l'élément mâle pouvant être remplacé par un " excitant de croissance " quelconque. Et c'est ainsi que, suivant les méthodes 
et les techniques plus ou moins bien appropriées et perfectionnées, le développement des ovules non fécondés a pu être mené jusqu'aux stades de morula, de gastrula, de larve ou de têtard. Delage a pu même obtenir de cette façon des oursins adultes.

Ces recherches, bien qu'entreprises dans un but tout autre que celui qui nous préoccupe, ont permis de constater qu'en faisant agir sur les œufs d'oursins des substances cytolytiques quelconques, des acides organiques: butyride, acétique ou des bases faibles: de la saponine, solanine, des sels biliaires ou des savons, des hydrocarbures (benzène, toluène), de l'alcool ou de l'éther en très petite quantité et pendant très peu de temps), on provoque la dissolution de la couche périphérique superficielle de l'œuf (formation de la membrane de fécondation). Les œufs ainsi traités et replacés ensuite dans leur milieu normal, continuent à se développer comme s'ils avaient été fécondés par leurs spermatozoïdes spécifiques. Mais, si l'on fait agir les mêmes substances à doses un peu plus fortes, ou un peu plus longtemps, ou encore à une température supérieure à $15^{\circ}$, on obtient la destruction des œufs par dissolution complète de toute leur masse protoplasmique.

Loch a constaté encore, qu'on peut empêcher la cytolyse complète des œufs d'oursin et obtenir leur développement jusqu'au stade de Pluteus à une température supérieure à $15^{\circ}$, en les plongeant, après le traitement par un acide, dans une solution hypertonique (eau de mer d'une concentration de 50 p. 100 supérieure à la normale) pendant 20 à 60 minutes, ou bien dans une eau de mer dépourvue d'oxygène dissous, ou encore dans une eau de mer normale à laquelle on a ajouté une trace de cyanure de po- 
tassium et en les reportant ensuite dans l'eau de mer normale. Dans ces cas, la solution hypertonique a donc produit le même effet que l'abaissement de température et il est facile de comprendre pourquoi il doit en être ainsi: en abaissant la température on ralentit l'action de l'acide sur le plasma, et une solution hypertonique provoque le même résultat en retirant un peu d'eau contenue normalement dans la cellule. Or, comme on peut produire encore le même effet en privant les ovules d'oxygène ou en ajoutant au milieu du cyanure de potassium, on peut en déduire, que l'action de tous ces facteurs consiste dans l'arrêt momentané du processus d'oxydation.

On sait, d'autre part, que la plupart de ces substances (excepté les bases) qui, suivant les conditions de concentration, de temps et de température, maintenues dans certaines limites, exercent sur la cellule tantôt une action de stimulation ou développante, par dissolution partielle, tantôt une destruction par dissolution complète, peuvent provoquer la mort de la cellule par une coagulation plus ou moins complète de son plasma, quand on les fait agir en concentrations beaucoup plus fortes.

Ainsi, la même substance, un acide ou un alcool, par exemple, agissant sur l'ensemble d'une cellule vivante, peut provoquer deux phénomènes diamétralement opposés: une excitation formatrice par dissolution partielle, ou bien une destruction complète par dissolution ou par coagulation de la masse totale du plasma.

On peut obtenir des résultats analogues en faisant agir un antiseptique, par exemple un sel de mercure 
ou de l'acide arsénique sur les organismes unicellulaires, et en particulier sur des bactéries qui se multiplient rapidement par divisions successives et qu'il est facile d'étudier en les cultivant dans des milieux nutritifs artificiels. Voici l'expérience:

Action de l'acide arsénique sur la culture de la bactéridie du charbon. - On distribue dans une série de 50 tubes, par parties égales, une série de solutions d'acide arsénique $\left(\mathrm{AsO}^{5}\right)$ allant de 1 p. 10.000 à 1 p. 200, dans du bouillon nutritif (bouillon de viande additionné de 1,5 p. 100 de peptone neutre); on ensemence chaque tube avec une goutte de culture fraîche de bactéridie charbonneuse non sporulée et on porte les tubes ainsi ensemencés à l'étuve à $37^{\circ}$. On ensemence en même temps, dans les mêmes conditions, quelques tubes contenant du bouillon sans acide arsénique. Ce seront des tubes témoins.

Après un séjour de 48 heures à l'étuve, on exatmine tous ces tubes et on trouve, en commençant par les dilutions les plus faibles:

Dans les solutions de 1 p. 10.000 à 1 p. 5.000 (tubes $n^{\circ s} 1$ à 3), la culture est sensiblement moins abondante que dans les tubes témoins;

Dans les solutions de 1 p. 3.500 à 1 p. 1.450 (tubes $\mathrm{n}^{\circ s} 4$ à 7 ), la culture est sensiblement plus abondante que dans les tubes témoins;

Il n'y a pas de culture du tout dans les solutions de 1 p. 1.250 à 1 p. 500 (tubes 8 à 20 );

Dans les solutions encore plus concentrées ( 1 p. 400 à 1 p. 200), on trouve un précipité (tubes 21 à 50 ).

On peut contrôler les résultats de l'expérience précédente en ensemençant quelques gouttes du contenu de chacun de ces tubes sur gélose (agar-agar) en tubes inclinés. On trouve alors des colonies moins 
nombreuses dans les tubes 1 à 3 que dans les tubes 4 à 7 . Il n'y a pas de culture du tout dans les tubes 8 à 20 , mais on trouve des colonies dans les tubes de gélose 21 à 25, tandis qu'il n'y avait pas de développement de microbes dans les tubes correspondants de bouillon. A partir du tube $n^{\circ} 26$, les tubes de gélose restent stériles.

L'examen microscopique du contenu des tubes de bouillon donne d'autres renseignements intéressants: on trouve des microbes vivants dans les tubes $n^{\circ s} 1$ à 10 (solutions 1 p. 10.000 à 1 p. 3.000), ainsi que dans les tubes 20 à 25 ; on trouve aussi des corps microbiens morts, mais bien conservés dans les tubes 26 à 50. On ne trouve rien dans les $n^{\circ s} 10$ à 20 , et on doit en conclure, que dans ces derniers tubes les microbes ont été tués par dissolution.

Quand on tue ces mêmes microbes par la chaleur (en les plongeant pendant une heure dans un bainmarie chauffé à $100^{\circ}$ ) et quand on les soumet ensuite à l'action des solutions d'acide arsénique de 1 p. 1.250 à 1 p. 500 (tubes $n^{\circ 8} 10$ à 20 ), on voit les corps microbiens se conserver sans aucune modification apparente. On doit en conclure que, dans ces tubes 10 à 20 , les microbes n'ont pas été dissous par l'acide arsénique, mais qu'il y a là un phénomène d'auto-digestion ou d'autolyse. On peut dire que, dans ce cas, l'acide arsénique a arrêté les fonctions de synthèse sans arrêter celles de décomposition des microbes, tandis qu'une quantité double de cet acide (solutions de 1 p. 500 à 1 p. 400) a eu pour effet d'arrêter momentanément ces deux fonctions sans tuer les microbes. Un séjour un peu plus long dans les solutions de 1 p. 480 à 1 p. 400 a pour résultat la mort des microbes par coagulation. 
Ainsi, on constate que la solution moyenne de 1 p. 7.000 ralentit la multiplication des microbes, celle de 1 p. 3.000 d'acide arsénique excite leur croissance, celle de 1 p. 1.000 arrête l'assimilation sans arrêter l'auto-digestion et tue les microbes par dissolution, celle de 1 p. 480 paralyse les deux fonctions. sans tuer les microbes, et enfin celle de 1 p. 400 les tue en coagulant le plasma. Pour chacune de ces réactions, il y a un "optimum" de concentration ou de quantité d'acide fixé par les éléments de la cellule, et il est important de noter ici que les effets d'accélération ou de ralentissement de la multiplication des microbes, ainsi que de leur destruction par hydrolyse totale, sont provoqués non pas par les éléments directement touchés et détruits, mais par les éléments encore vivants, c'est-à-dire que l'effet appréciable est le résultat d'une réaction indirecte de la partie vivante de la cellule.

L'étude de l'action des solutions hypertoniques sur des animaux supérieurs ou sur des organismes unicellulaires, peut nous montrer les effets de ces réactions encore sous un autre jour.

En injectant à une souris de 20 gr., sous la peau du dos, 1 c. c. d'une solution de sel marin à 10 p. 100 , soit dix centigrammes de sel, ce qui fait un $200^{\circ}$ du poids de l'animal, on constate qu'au bout de dix minutes sa respiration devient de plus en plus fréquente. Après 30 minutes, l'animal tombe sur le flanc, est agité de soubresauts et succombe peu après avec tous les signes d'asphyxie.

A l'autopsie on trouve les reins un peu congestion- 
nés, mais l'aspect à peu près normal de tous les autres organes ne justifie nullement une mort aussi rapide. Dans la région injectée on trouve un œdème contenant un liquide jaunâtre, ce qui prouve qu'un peu de plasma et de lymphe est venu se mélanger à la solution saline.

$\mathrm{Si}$, au lieu de 10 centigr. de sel en une seule fois on en injecte 12 ou même 13 centigr. par fractions de 3 centigr. à des intervalles de 30 minutes, toujours dans le même volume total d'eau (1 c. c. en tout), la souris n'en souffrira aucunement sur le moment et continuera à vivre normalement pendant des semaines et des mois.

Ainsi, on constate qu'à la dose de un deux-centième du poids de l'animal, le chlorure de sodium n'est toxique qu'à la condition d'être injecté en solution concentrée et d'intéresser directement et rapidement une certaine étendue du corps de l'animal. La même quantité et même une quantité supérieure ne sera pas toxique, quand l'injection sera faite assez lentement pour que le sel puisse diffuser au fur et à mesure dans le corps tout entier.

Pour examiner l'aetion toxique du sel marin avec un peu plus de détails, on en injecte à un cobaye de $365 \mathrm{gr}$., sous la peau du ventre, 1 gr. 800 en solution dans 9 c. c. d'eau distillée, soit un deux-centième de son poids.

L'injection ne semble provoquer aucune douleur. La température du cobaye prise quelques minutes avant l'injection est de $38^{\circ} 2$.

Après l'injection, on prend la température de l'animal toutes les 30 minutes et on constate un abaissement de $2^{\circ}$ au bout de la première demi-heure et ensuite un abaissement presque régulier chaque fois 
d'un demi-degré, de sorte qu'au bout de trois heures, quelques minutes avant la mort de l'animal, on ne note plus que $33^{\circ}$.

Il n'est guère possible de suivre directement les réactions qui se passent dans les cellules de la souris ou du cobaye ainsi traité, entre le moment de l'injection du sel et la mort de l'animal. Dans les organismes aussi complexes, les modifications cellulaires constatées à l'autopsie, après la mort de l'animal, peuvent résulter non pas de l'action directe du sel sur la cellule lésée, mais d'une série de réactions réflexes, que nous ne connaissons pas. L'action directe d'une solution d'un sel qui agit uniquement par sa concentration et non par une propriété toxique élective, peut être étudié plus facilement sur des êtres unicellulaires, sur des infusoires nageant dans une goutte d'eau et en observant ce qui se passe au microscope.

Les paramécies se prêtent entre autres très bien à une telle étude.

On fait tomber sur une lame de verre une goutte d'eau contenant quelques paramécies et, à côté, une goutte d'une solution de sel à 10 p. 100 . On réunit alors les deux gouttes. Il s'établira entre les deux gouttes deux courants en sens inverse: l'eau passera dans la solution saline et le sel dans l'eau, de sorte qu'au bout de quelques minutes il y aura une quantité égale de sel et d'eau dans les deux gouttes. Les paramécies sentiront l'arrivée du sel. Elles seront d'abord un peu plus agitées, ensuite leurs mouvements deviendront plus lents et s'arrêteront complètement. Leur corps ne changera pas de forme tout d'abord, mais le plasma deviendra plus granuleux et, en même temps que l'arrêt du mouvement, on consta- 
tera un rétrécissement du plasma et des plissements de la membrane. Le corps perdra bientôt environ un tiers de son volume, et, si on le laisse ainsi pendant quelques minutes, il ne reviendra plus à la vie.

Dans l'organisme de la souris comme dans celui du cobaye, il y a certainement aussi une action purement physique du sel en solution concentrée sur les cellules environnantes, comme on vient de le voir pour les paramécies. Ces cellules sont déshydratées parce que le passage de l'eau intracellulaire dans la solution saline est plus rapide que le passage du sel dans les cellules, mais dans ce cas la déshydratation d'un certain nombre de cellules directement touchées par la solution saline n'a pu causer la mort aussi rapide de l'animal, que par une répercussion de ce trouble local sur l'économie générale. Cette mort survenue 15 à 25 minutes chez la souris, ou 3 heures chez le cobaye après l'injection, avec des symptômes d'asphyxie, n'a pu être causée que par une réaction nerveuse sur le processus d'oxydation.

Il est donc à noter ici que le sel marin qui n'est pas un élément étranger à l'organisme, puisque chaque organisme en contient des quantités notables, et qui n'est pas un poison, à proprement parler, peut, malgré cela, devenir très pathogène, quand il est introduit rapidement dans une région limitée mais encore assez étendue de cet organisme. Il n'agit que par la rapidité et l'étendue des troubles qu'il provoque, par une rupture d'équilibre que l'organisme n'a pas le temps de rétablir.

Pour trouver l'explication de ces phénomènes et analyser en détail le mécanisme des réactions dont 
nous venons d'enregistrer les résultats, on peut simplifier le problème en éliminant les facteurs qui agissent dans le même sens et peuvent, dans certaines limites, se remplacer mutuellement.

Ainsi, nous avons vu plus haut, que la même dose d'un acide peut produire deux résultats contraires : une stimulation du développement ou une destruction complète et rapide de l'œuf d'oursin, suivant la durée de l'action de l'acide ou la température à laquelle a été faite l'expérience. En faisant agir l'acide pendant 5 à 6 minutes à une température de $15^{\circ}$, l'œuf se développera, mais si on élève la température ou si on augmente la durée de l'action de l'acide, l'œuf sera détruit par dissolution (cytolyse) du plasma. On peut obtenir aussi le développement de l'œuf par une dose d'acide plus forte, mais à la condition de diminuer en même temps la durée de son action, de sorte qu'en opérant toujours dans des conditions de temps et de température identiques, on n'aura à tenir compte que d'un seul facteur, "la dose ", ou la concentration de l'acide.

Pour apprécier la nature et le mécanisme des réactions, il suffira donc de déterminer la dose de la substance agissante, parce que, en réalité, ce qui agira, ce n'est pas la quantité de substance active versée dans le liquide de culture ou injectée à un animal, mais uniquement la quantité fixée par les cellules, en un temps donné, et ainsi que nous venons de le voir, cette quantité dépend de la concentration des liquides, de la durée du contact et de la température.

Tout ceci, bien entendu, dans certaines limites; à une température trop basse aucune réaction vitale ne se produira, quelles que soient la nature et la

DAnysz. - La genèse de l'énergie psychique. 
quantité de la substance agissante, et une température de 55 à $60^{\circ}$ coagulera le plasma sans intervention d'aucune substance étrangère.

Une première analyse nous conduit donc à admettre, que la nature de la réaction (stimulation ou destruction) provoquée dans une cellule par une solution active, dépend de la quantité de cette substance fixée par la masse cellulaire, ou en d'autres termes, de la proportion de cette masse altérée dans sa composition et ses fonctions par les combinaisons qu'elle a formées avec cette substance étrangère.

Or, nous avons vu qu'une cellule est formée d'un grand nombre de micelles, qui sont ses unités de constitution physico-chimiques et de fonction. Ce sont aussi les unités de réaction de la cellule. L'action d'une substance étrangère sur une cellule dépendra donc de la proportion des micelles atteintes. Supposons qu'une cellule soit f̣ormée de 100.000 micelles, si on détruit 100 de ces micelles, la vie de l'ensemble de la cellule n'en souffrira pas sensiblement, au contraire, la mise en liberté de certains catalyseurs par une hydrolyse ou une coagulation partielle exercera une action stimulante sur le processus de nutrition et d'oxydation; - mais si on en détruit 30 ou 50.000 , la cellule succombera. On peut en dire autant d'une micelle formée de milliers de molécules: sa fonction de nutrition sera stimulée ou arrêtée suivant la quantité des molécules qui auront formé des combinaisons nouvelles, et apporteront ainsi une perturbation plus ou moins profonde dans son équilibre physico-chimique et dans son fonctionnement normal.

Ceci ne s'applique, bien entendu, qu'à des substances qui agissent surtout par leur concentration. 
hypo- ou hypertonique, par rapport à la tonicité de la matière vivante.

Le nitrate d'argent, par exemple, provoquera la destruction des infusoires par autolyse à une concentration infiniment plus faible que le chlorure ou le nitrate de sodium.

Dans ces cas, la nature de l'action excitante ou pathogène sera toute autre, et à mesure que nous chercherons à provoquer des réactions stimulantes ou nocives chez des êtres de plus en plus élevés, pourvus d'un système nerveux de plus en plus différencié et centralisé, nous verrons parallèlement augmenter la liste de ces substances électives, produisant des effets considérables par des doses relativement minimes.

La proportion des micelles atteintes dans une cellule, des cellules atteintes dans un organisme pluricellulaire, n'est donc pas le seul facteur, dont il faut tenir compte pour comprendre les diverses modalités de l'action stimulante ou pathogène des différents cristalloïdes sur les êtres vivants. Il faut encore prendre en considération les effets, que peuvent produire les différentes substances étrangères sur les différentes fonctions de l'organisme, ou, en d'autres termes, l'importance du rôle de différentes micelles dans la vie d'une cellule, de différentes cellules ou tissus dans la vie d'un organisme.

Ainsi, le cyanure de potassium sera excitant ou toxique à des doses beaucoup plus faibles que le chlorure, le nitrate ou le sulfate de potassium, parce que le premier agira sur les réactions d'oxydation d'une façon beaucoup plus directe et plus forte que le chlorure ou le sulfate et que les réactions d'oxydation et de réduction sont beaucoup plus impor-

\section{http://rcin.org.pl}


tantes pour la vie de l'individu que les réactions de nutrition ou de reproduction. La strychnine ou la morphine sont des excitants et des poisons cent fois plus énergiques pour des organismes pourvus d'un système nerveux différencié que la quinine, parce qu'ils sont névrotropes, possèdent une affinité et exercent une action spéciale sur les cellules des centres nerveux, tandis que la quinine ne semble pas agir d'une façon aussi élective sur ce tissu.

Les organismes supérieurs peuvent vivre plusieurs jours et même plusieurs semaines sans s'alimenter, tandis qu'un arrêt de la respiration pendant quelques minutes met leur vie en danger. De même, les lésions même étendues du tissu épithélial ou musculaire sont infiniment moins dangereuses que les lésions de quelques cellules du bulbe.

L'étude du mécanisme apparent des réactions provoquées par les cristalloïdes est d'autant plus difficile, que l'organisme soumis à l'expérience est plus complexe.

Chez l'homme, on peut guérir une bronchite ou une douleur rhumatismale en badigeonnant la peau de la poitrine, du dos ou d'une articulation, avec de la teinture d'iode, ou bien par des vésicatoires ou des ventouses ou encore par la chaleur. On provoque ainsi des réactions dans les cellules de l'épiderme, mais il est impossible d'admettre que ce sont les combinaisons de l'iode avec les substances des eellules épidermiques ou l'oedématisation de ces eellules par un vésicatoire, qui fait disparaître, par une action directe, la congestion des bronches, séparées de la peau par plusieurs barrières infranchissables. Il est bien plus probable que les modifications chimiques provoquées dans les cellules de 
l'épiderme et surtout dans les cellules des terminaisons nerveuses, réagissent soit indirectement par l'intermédiaire des centres nerveux, soit plus directement par une réaction des substances résorbées, sur les produits des glandes à sécrétions internes, ainsi qu'on peut l'admettre d'après les recherches des physiologistes modernes sur les hormones.

Or, si on peut obtenir le même résultat apparent par l'iode, par une ventouse, un vésicatoire ou par l'air chaud, qui, sans aucun doute, provoquent dans les cellules de la peau des réactions chimiques différentes, il devient évident que la seule connaissance du mécanisme chimique des combinaisons qu'un réactif peut former localement dans les cellules, ne nous apprendra rien de précis sur le mécanisme des multiples réactions réflexes, qui aboutissent en définitive à un effet curatif ou nocif, stimulant ou destructif.

L'étude du mécanisme intime de toute réaction des tissus vivants est bien du domaine de la chimie, mais pour apprendre, par exemple, comment l'iode agit sur les processus congestifs, l'antipyrine ou la morphine sur la douleur, etc., il faudrait connaître les lois ou tout au moins les règles de la chimie des albuminoïdes, dont nous commençons à peine à entrevoir l'existence.

Au point de vue qui nous préoccupe ici : du rôle des cristalloïdes étrangers dans l'évolution progressive des êtres vivants, nous n'avons à envisager que leur action à doses stimulantes, - et comme nous n'en connaissons pas le mécanisme intime, nous devons nous borner à en indiquer les règles générales d'après les effets apparents qu'ils produisent. 


\section{Résumé}

Ces règles générales peuvent être résumées de la façon suivante :

Les réactions provoquées par les cristalloïdes dans les organismes sont rapides et pour ainsi dire immédiates, parce que ces substances traversent facilement les membranes cellulaires et peuvent former des combinaisons chimiques immédiates avec les substances contenues dans les cellules.

Facilement absorbées par l'organisme, les cristalloïdes sont aussi facilement et rapidement éliminés. Absorbés une ou même plusieurs fois, ils provoquent des modifications individuelles peu durables. Un cristalloïde ne peut provoquer des modifications héréditaires, qu'à la condition d'être absorbé habituellement pendant une longue série de générations et, dans ce cas, la modification stabilisée n'est pas l'effet immédiat de la réaction chimique, qui se produit entre la substance étrangère et la substance celluraire, mais d'un ensemble de réactions indirectes de l'organisme provoquées par la rupture de l'équilibre physico-chimique local. On peut admettre, en effet, que s'il y a une rupture d'équilibre après chaque intervention du cristalloïde étranger, l'organisme ne tarde pas à retrouver son équilibre antérieur normal aussitôt que la substance étrangère est éliminée. $\mathrm{Au}$ contraire, un équilibre nouveau peut s'établir et devenir durable si la même excitation se répète pendant un temps suffisamment long pour provoquer une modification durable dans les fonctions habituelles d'un organe quelconque. 


\section{8. - Réactions provoquées par les colloïdes}

Les réactions provoquées par les substances colloïdales sur les organismes vivants sont d'une toute autre nature, que celles provoquées par les cristalloïdes, et pour comprendre ce que cette action a de particulier et de distinctif, il faut se rappeler que les colloïdes inertes d'origine biologique ou inorganique ont pour unités de réaction des micelles d'une composition et d'une structure analogues à celles des micelles des substances albuminoïdes vivantes.

Or, de même que les molécules composées des sels ne peuvent réagir les uns sur les autres qu'après avoir été dissociées en atomes, une micelle inerte ne pourra agir sur une micelle vivante, qu'après avoir été décomposée en molécules, qui seules peuvent former des combinaisons avec les molécules de la micelle vivante.

Il faut done qu'une micelle inerte soit digérée, pour que les éléments simples qui la composent, puissent être assimilés par une micelle vivante.

Chez les êtres pluricellaires, la digestion des colloïdes s'opère par des organes et cellules spécialement adaptés à cette fonction: par les racines garnies de radicelles chez les plantes, par les muqueuses du tube digestif, qui n'est, au fond, qu'un appareil radicellaire invaginé, chez les animaux;

(1) En faisant agir une molécule de chlorure de sodium $(\mathrm{Na} \mathrm{Cl})$ sur une molécule de nitrate d'argent $\left(\mathrm{Ag} \mathrm{AzO}^{3}\right)$, on obtiendra une molécule d'azotate de sodium $\left(\mathrm{Na} \mathrm{AzO}{ }^{3}\right)$ et une de chlorure d'argent $(\mathrm{Ag} \mathrm{Cl})$, mais pour que cet échange soit possible, il faut que les molécules $\mathrm{Na}, \mathrm{Gl}, \mathrm{Ag}$ et $\mathrm{Az}$ deviennent libres, ou qu'elles deviennent des ions. La dissociation des sels dans l'eau est un phénomène en tout comparable à l'hydrolyse ou, autrement dit, à la digestion des micelles. 
mais aussi bien pour les êtres pluricellulaires que pour les cellules libres, la transformation des micelles non assimilables en molécules assimilables se fait normalement toujours en dehors des cellules. Cette opération est effectuée par des catalyseurs ou ferments digestifs excrétés par les cellules dans le milieu liquide qui les entoure. Seuls les cristalloïdes devraient pénétrer normalement dans le milieu intérieur des organismes pluricellulaires et à l'intérieur des cellules.

Il arrive pourtant, qu'à la faveur d'accidents ou d'événements de toutes sortes, des substances colloïdales s'introduisent dans le milieu intérieur d'un organisme pluricellulaire ou d'une cellule, et alors, pour comprendre ce qui va se passer, il faut distinguer et considérer plusieurs cas particuliers, et notamment :

$1^{\circ}$ La composition chimique et.la structure des micelles du colloïde absorbé peuvent être identiques à celles des micelles de la cellule qui les absorbera. Ce sont les albumines homo- ou autogènes provenant des humeurs, tissus ou organes d'añimaux de la même espèce ou du même individu.

$2^{\circ}$ La composition chimique élémentaire peut être la même, mais la structure des micelles sera différente par l'arrangement et les proportions des molécules simples et complexes.

Ce sont des albuminoïdes hétérogènes, provenant des humeurs, tissus et organes d'animaux ou de végétaux appartenant à des espèces différentes de l'animal qui les a absorbés.

$3^{\circ}$ Les micelles des colloïdes absorbées peuvent différer de celles de l'organisme qui les absorbe, non seulement par leur structure générale, mais aussi 
par la composition élémentaire des molécules qui les composent. Ce seront des colloüdes étrangers.

Les colloïdes des deux premières catégories sont alimentaires, les molécules qui composent leurs micelles peuvent entrer telles quelles dans les cellules de l'organisme qui les a digérées et être assimilées, elles ne seront pas nocives. Les molécules des colloïdes étrangers seront indifférentes ou nocives, mais en outre de ces réactions nutritives ou nocives provoquées par les colloïdes décomposés, c'est-à-dire par les éléments, les sels et les cristalloïdes devenus libres, les collö̈des introduits dans le milieu intérieur d'un organisme ou d'une cellule agiront d'une façon particulière par leur nature même, parce qu'ils ne peuvent pas être directement assimilés ni éliminés et parce qu'ils sont digérés dans le milieu intérieur, qui n'est pas adapté à cette fonction (1).

Cette question n'a été étudiée, jusqu'à présent, avec quelque précision que chez les animaux supérieurs et notamment chez les mammifères.

1. Anaphylaxie. - Ainsi, on sait que les albumines animales ou végétales peuvent être mangées par les animaux sans aucun inconvénient pour leur santé et que ce sont là des substances alimentaires habituelles, dont les animaux ne peuvent pas être privés, sans mourir de faim. Mais si on injecte ces mêmes substances, par exemple, du sérum de cheval ou du blanc d'œuf sous la peau ou dans le sang à un chien, à un lapin ou à un homme, ou bien, si ces

(1) Les colloïdes sont complètement insolubles dans l'eau. Les molécules qui composent les micelles ne peuvent done etre dissociées et réagir sur d'autres molécules qu'à la condition de devenir libres et solubles dans l'eau. 
substances non digérées ou n'ayant subi qu'une digestion incomplète passent dans le sang à travers la muqueuse intestinale ou trachéale à la faveur d'une congestion accidentelle de cette muqueuse, alors elles provoquent dans les cellules et dans la composition des humeurs de l'animal certaines modifications durables, et ce qui est plus particulièremènt important à noter, toujours les mêmes dans l'ensemble, sinon dans tous leurs détails, quelle que soit la nature, l'origine et la composition chimique du colloïde, que ce soit une albumine alimentaire, une toxalbumine (certains venins, sérum d'anguille ou de murène, ete.) ou une substance colloïdale préparée par synthèse (arsénophénylglycine, .arsénobenzène, etc.).

Quelles sont ces modifications, par quelles manifestations apparentes se traduisent-elles, quel en est le mécanisme et la raison d'être?

Les recherches de biologie et de médecine expérimentale de ce dernier demi-siècle nous permettent de répondre à toutes ces questions d'une façon suffisamment claire et précise.

Hayem (1), le premier, a eu l'idée (en 1889) d'injecter dans la veine d'un chien du sang de bœuf et de répéter cette opération treize jours après. Il a constaté que le chien qui a supporté la première injection de 48 centilitres, sans aucun trouble apparent, a succombé à la seconde injection d'une dose moins forte, après une série de manifestations pathologiques violentes : prurit, dyspnée, diarrhée, vomissements, convulsions, etc.

L'autopsie du cadavre a révélé des congestions et

(1) HAYEM, Du sang et des altérations anatomiques, Paris, 1889. 
des hémorragies capillaires nombreuses dans presque tous les organes; le sang est devenu incoagulable et, conservé pendant 24 heures, il a laissé déposer un précipité granuleux.

La première injection a donc profondément modifié la composition et, ce qui s'en est suivi, les propriétés de certaines cellules et du liquide sanguin.

Belfanti et Carbone, J. Bordet, Kraus et beaucoup d'autres expérimentateurs ont montré quelques années plus tard (1896-1900), en procédánt de la même façon, mais en variant les conditions des expériences, que le sérum d'un animal injecté avec une substance albuminoïde hétérogène quelconque et qui ne précipitait pas une dilution de cette albumine avant l'injection, acquiert 10,15 ou 20 jours après cette injection, la propriété de former un précipité quand on le mélange avec elle dans un verre. La première injection a done provoqué dans l'organisme de l'animal injecté la formation d'une substance nouvelle qui a, elle aussi, les propriétés d'une albumine et qui possède une affinité spéciale pour la substance injectée. Cette substance, appelée anticorps, qui n'apparaît dans le sang de l'animal injecté qu'après une période de temps plus ou moins longue (12 à 20 jours) après l'injection, ne peut être qu'une production ou sécrétion de certaines cellules.

Les substances qui provoquent la formation des anticorps ont été appelées: antigènes, et on a constaté que les anticorps et les antigènes sont réciproquement spécifiques : le sérum de cheval injecté à un lapin, provoque dans l'organisme du lapin injecté la formation d'un anticorps qui donne un précipité quand on le mélange avec le sang normal d'un cheval quelconque, mais ne réagit pas de la même façon 
avec le sang des animaux d'autres espèces; de même, l'anticorps préparé pour le blanc d'œuf de la poule, ne réagira pas ou, en tout cas, pas avec la même force, avec le blanc d'œuf du canard ou de l'oie.

Quelles sont les cellules de l'organisme injecté avec un antigène qui participent plus spécialement à la production des anticorps ?

Pour Metchnikoff, ce rôle serait dévolu exclusivement aux leucocytes, et il est facile de constater, qu'en effet, les globules blancs du sang englobent et digèrent tout corpuscule microscopique étranger vivant ou mort qui a pénétré dans le sang ou dans les tissus. On peut donc admettre qu'ils absorbent et digèrent de la même façon les micelles des substances albuminoïdes hétérogènes; mais, en admettant qu'il en soit ainsi, il serait difficile d'expliquer les manifestations pathologiques qui suivent les injections de ces albumines.

En effet, des injections peu nombreuses de petites doses de ces substances ne provoquent généralement pas de modifications appréciables et qu'il serait facile à constater à l'autopsie; mais chez les animaux, qui ont reçu un très grand nombre d'injections de toxines, de corps microbiens, de sang ou de sérum hétérogène, on constate des modifications profondes dans la rate et dans la moelle osseuse, des lésions dans le foie et les reins, des hypertrophies et des dégénérescences dans presque toutes les glandes à sécrétions internes (surrénales, thyroïdes hypophyse, pancréas).

Il n'est guère possible d'attribuer ces lésions à l'empoisonnement des cellules de ces glandes, puis- 
qu'on obtient les mêmes effets par des colloïdes alimentaires, dont les molécules ne sont pas des poisons, il faut bien en chercher les causes dans une réaction des cellules des glandes lésées: les attribuer au travail supplémentaire de la production EN ExcÈs des anticorps, destinés à la digestion des antigènes, c'est-à-dire des colloüdes.

L'introduction des antigènes dans le milieu intérieur de l'organisme provoque donc des modifications de deux sortes:

$1^{\circ}$ Congestion et hypertrophie qui peut aller jusqu'à une dégénérescence plus ou moins étendue de certains organes et glandes, qui produisent l'excès d'anticorps, et qui deviennent "insuffisants" pour exercer leurs fonctions normales.

$2^{\circ}$ Présence de cet excès d'anticorps dans le sang et les humeurs de l'organisme.

Les symptômes par lesquels ces modifications se manifestent sont aussi de deux sortes:

$1^{\circ}$ Les insuffisances glandulaires auront pour conséquence un trouble plus ou moins grave de la nutrition générale, qui se traduira en dernier lieu par les symptômes qui caractérisent toutes les affections chroniques connues (1): troubles gastro-intestinaux, dermatoses, rhumatismes, asthme et emphysèmes, neurasthénie, psychasthénies, épilepsie, etc...

$2^{\circ}$ La présence d'un excès d'anticorps dans la circulation provoquera des crises plus ou moins vio-

(1) On doit en excepter celles, bien entendu, qui résultent de traumatismes accidentels ou de malformations congénitales, bien que, dans ces derniers cas, le mal hérité doit avoir pour cause l'anaphylaxie des parents. 
lentes, parfois mortelles, chaque fois qu'une certaine quantité d'antigène viendra se mélanger dans le sang avec l'anticorps en excès et provoquera la formation d'un précipité (1).

Le degré de violence de la crise, la rapidité avec laquelle elle apparaît, sa durée, sa gravité, dépendent des quantités absolues et des proportions de l'antigène et de l'anticorps, qui se trouveront en présence dans le sang et dans les humeurs.

Ceci dit, l'explication du mécanisme et de la raison d'être de ces réactions apparaît, pour ainsi dire, d'elle-même.

On peut bien admettre aujourd'hui, que tous les êtres vivant actuellement proviennent, par une série d'évolutions, d'êtres unicellulaires qui, primitivement, se nourrissaient exclusivement de sels inorganiques. Certaines de ces cellules, placées dans des milieux riches en produits de décomposition d'autres cellules semblables, ont pris l'habitude de se nourrir, c'est-à-dire d'assimiler d'abord ces produits de décomposition (sels et cristalloïdes organiques) et ensuite de concourir à la décomposition des substances albuminoïdes des cadavres, en sécrétant des ferments digestifs.

Chez les organismes pluricellulaires, il s'est établi peu à peu une différenciation et une spécialisation des fonctions et des structures des cellules en différents tissus. Ce sont les cellules des radicelles chez les plantes, des muqueuses du tube digestif chez les animaux, qui seules ont gardé la propriété de pro-

(1) Les toxines (tétaniques et diphtériques font exception à cette règle très générale. Les mélanges des toxines et de leurs anticorps ne donnent jamais de précipités in vitro et ne provoquent pas de crises pathologiques in vivo. 
duire et de sécréter les ferments digestifs et l'ont même exagérée. Elles produisent les ferments digestifs (les anticorps) en excès, c'est-à-dire plus qu'il n'en faut pour leurs propres besoins.

Les cellules de tous les autres tissus, nourries normalement, exclusivement de cristalloïdes, ont perdu l'habitude de produire ces anticorps, mais elles en ont gardé plus ou moins le "souvenir". Les globules blancs du sang (phagocytes de Metchnikoff) possèdent cette capacité au plus haut degré, les cellules nerveuses en sont probablement complètement dépourvues.

Aussi, quand les cellules du milieu intérieur viennent en contact avec une substance colloüde, ce "souvenir" se réveille à nouveau et elles commencent à produire des ferments digestifs ou autrement dit des anticorps en excès. Quand on intervient pour la première fois, la production des anticorps est très lente, le sérum de cheval injecté dans le sang du lapin, par exemple, circule dans les vaisseaux sanguins du lapin pendant 10,15 ou 20 jours. On le retrouve à l'aide de certaines réactions spéoifiques; mais les cellules chargées de la production des anticorps, une fois mises en train, continuent cette production. Bientôt tout le sérum de cheval sera digéré et il y aura des anticorps en excès dans le sang et les humeurs.

L'animal injecté semble en parfait état de santé, mais si, à ce moment on lui injecte une nouvelle quantité de sérum de cheval dans la veine, l'opération de la digestion commencera très rapidement et la première phase de cette digestion sera la formation d'un précipité, qui provoquera des troubles vasculo-sanguins presqu'immédiats. 
Les cellules des glandes et autres tissus du milieu intérieur, producteurs des anticorps en excès, en souffrent parce qu'elles ne sont pas "libres ", parce qu'elles font partie d'un milieu fermé, dont toutes les unités sont et doivent être parfaitement accordées pour un travail, dont la qualité et la quantité est bien déterminée pour les besoins de l'ensemble. Le trouble d'équilibre d'une de ces unités se répercutera sur toutes les autres, l'insuffisance fonctionnelle d'une glande réagira directement ou indirectement, par l'intermédiaire du système nerveux, sur la nutrition générale qui deviendra anormale, et il en résultera des manifestations variées suivant les différences des prédispositions ou "diathèses » individuelles, héréditaires ou acquises.

Ce trouble de nutrition générale durera aussi longtemps que la surproduction des anticorps: des semaines, des mois, des années ou même des dizaines d'années suivant la nature (encore inconnue) des antigènes.

La raison d'être de l'action pathogène uniforme des colloïdes est donc l'obligation pour les cellules du milieu intérieur de l'organisme, de les digérer, grace au réveil d'une fonction ancestrale qu'elles n'ont pas complètement perdue, mais à laquelle le milieu intérieur de l'organisme n'est pas adapté.

L'introduction des microbes vivants dans le milieu intérieur de l'organisme représente un cas particulier des réactions provoquées par les colloïdes.

Les êtres unicellulaires (microbes, levures, champignons), habitués à vivre tout d'abord dans des milieux riches en produits cristalloïdes provenant de 
la décomposition des albumines (décomposition des cadavres) ont pu ensuite s'adapter au travail de décomposition de ces albumines, c'est-à-dire à secréter des ferments digestifs et à vivre en parasites dans les milieux vivants. Ils peuvent alors agir:

$1^{\circ} \mathrm{Par}$ leurs excrétions qui sont généralement cristalloïdes et peuvent être pathogènes, nutritives ou indifférentes comme des cristalloïdes d'origine quelconque;

$2^{\circ}$ Par leurs sécrétions et par les substances albuminoïdes qui composent leurs corps. Ces deux sortes de substances sont généralement des colloïdes et provoqueront toujours des troubles et des manifestations pathologiques par leur nature d'antigènes, en agissant soit à la fois directement et indirectement (toxines, diastases), soit seulement indirectement comme des albumines hétérogènes non toxiques.

2. Rôle des colloïdes dans l'évolution. - En résumé, et en ne considérant pour le moment que l'action antigénique des colloïdes, on peut dire qu'un organisme qui a subi une fois l'action d'un antigène et l'a décomposé ou digéré, aura acquis par cela même la faculté de le digérer une deuxième fois bien plus facilement et plus rapidement, grâce à la présence dans son sang et dans ses tissus d'une quantité plus grande de l'anticorps correspondant.

Cette faculté ainsi acquise aura toujours pour résultat de troubler plus ou moins profondément son équilibre nutritif général, mais lui procurera en même temps l'avantage de le rendre plus résistant à une réinfection dans les cas où l'antigène sera un microbe pathogène mort ou vivant (immunité par vaccination ou par une maladie spontanée guérie).

Daxysz. - La genèse de l'énergie psychique. 
En effet, dans les infections spontanées, le nombre des microbes infectants est généralement très petit et leur multiplication, surtout au début, très lente, si l'organisme (vacciné) possède, au moment de la néinfection un certain excès d'anticorps dans son sang et ses tissus, les microbes seront détruits (digérés) avant qu'ils n'aient eu le temps de se multiplier.

Pour l'histoire de l'évolution de nos connaissances dans le domaine de la biologie expérimentale et pour l'organisation future de ces recherehes, il est intéressant de noter ici, qu'on n'est arrivé à cette conception très simple du mécanisme de l'action des colloïdes, qu'après une somme de travail énorme. On cherchait avant tout à obtenir des résultats pratiques, aussi quand les travaux de Pasteur ont bien établi l'origine microbienne des maladies infectieuses, on s'est mis partout, au hasard des circonstances, à Ia recherche des moyens prophylactiques ou curatifs de ces maladies, sans se préoccuper des règles ou lois biologiques générales qui déterminent la nature, l'allure et le mécanisme de ces réactions. On a ainsi accumulé expériences sur expériences, qui donnaient. quelquefois des résultats pratiques importants, mais dont l'interprétation incomplète, parfois erronée, conduisait souvent sur de fausses routes ou aboutissait à des impasses.

C'est ainsi que l'on a découvert tout d'abord les phénomènes d'immunité "active et passive", ou, en d'autres termes, les principes de la vaccination préventive au moyen de cultures microbiennes atténuées ou stérilisées et de la sérumthérapie par des 
sérums spécifiques antitoxiques ou antimicrobiens, sans soupçonner ni se préoceuper autrement des suites d'autres natures, des troubles que ces interventions pouvaient avoir pour l'organisme.

Ce n'est que bien plus tard que l'on a reconnu la constance de ces troubles et qu'on a pu découvrir leur pathogénie et on a donné à ce phénomène le nom d'anaphylaxie (Ch. Richet), ce qui veut dire : contraire à la prophylaxie ou à l'immunité, sans se douter, tout d'abord que ces deux phénomènes procèdent de la même réaction, que ce n'est là qu'une question de doses ou de proportions d'antigène et d'antieorps mis en présence. Une petite dose de microbes vivants, injectée à un animal vacciné, sera détruite rapidement sans aucun symptôme morbide apparent: l'excès d'anticorps protégera l'animal contre une réinfection; une dose plus forte sera détruite aussi, grâce au même excès d'anticorps, mais alors la même réaction de destruction se traduira par une crise anaphylactique plus ou moins grave, parfois mortelle.

En réalité, il n'y a donc qu'un seul phénomène : production d'un anticorps en excès sous l'action d'une substance colloïdale hétérogène introduite dans le milieu intérieur de l'organisme. Il en résulte toujours un trouble plus ou moins profond dans la structure et le fonctionnement des organes et dans la nutrition générale des tissus. L'anaphylaxie ou l'allergie (Friedberger) doit être considérée par conséquent comme un phénomène général, la prophylaxie ou l'immunité acquise n'en est qu'un cas particulier, lorsque l'antigène est un microbe vivant pathogène, une toxine, une toxalbumine ou tout autre poison colloïdal. 
En outre de ces effets sur l'individu, les colloïdes organiques exercent une action beaucoup plus profonde sur l'évolution et la transformation des espèces que les cristalloïdes. Les hypertrophies qu'ils provoquent dans certaines glandes et les modifications dans les conditions de nutrition générale qui en résultent, mettent rarement, en effet, la vie des individus en danger immédiat. Elles sont, dans la plupart des cas, très durables et provoquent des réactions " de défense " dans les organes lésés, aussi bien que dans d'autres organes complémentaires.

On sait que, pour beaucoup de maladies infectieuses, l'immunité acquise par les parents se transmet à leur descendance et on sait aussi que les prédispositions morbides, les " diathèses ", qui ne sont que des états de sensibilité spéciale, sont, elles aussi, souvent héréditaires.

Or, cette sensibilisation peut avoir des degrés d'intensité différents et exercer sur l'ensemble de l'orgánisme une action de la même allure qu'une substance étrangère quelconque exerce sur une cellule. La lésion d'un tissu, d'un organe ou d'une glande peut être, suivant son intensité et son étendue, plus ou moins stimulante pour l'ensemble de l'organisme, plus ou moins pathogène ou plus ou moins rapidement mortelle et, en outre, suivant sa nature, elle peut influencer dans les mêmes sens, tantôt un organe, tantôt un autre, et déterminer, suivant l'importance de cet organe, pour l'économie générale, des modifications lointaines plus ou moins heureuses ou néfastes.

L'étude de ces phénomènes demanderait l'observation méthodique des familles pendant plusieurs générations, la vie d'un seul observateur n'y suffirait 
donc pas; on n'a pas songé, jusqu'à présent, à créer des institutions capables d'organiser et de poursuivre ces recherches pendant assez longtemps, on ne possède à ce sujet, que quelques notions isolées et incertaines.

Toutefois on a remarqué que la syphilis, par exemple, exerçait souvent, pendant certaines périodes de son évolution, une influence stimulante sur l'activité cérébrale des malades et que les enfants de syphilitiques se signalent souvent par une intelligence plus vive que la moyenne ou par des talents particuliers pour les arts ou les sciences. La fièvre typhoïde affaiblit quelquefois certaines facultés mentales, mais exerce souvent une influence heureuse sur la santé générale ou sur certains états morbides chroniques, tels que par exemple les rhumatismes, les sciatiques, le psoriasis, etc.

On peut admettre "à priori " que les enfants conçus pendant ces périodes de stimulation ou de dépression des deux parents ou de l'un d'eux, en bénéficient ou en souffrent, et il serait très intéressant pour l'avenir des races, de soumettre ce vaste territoire, encore très peu connu, à une exploration systématique.

Bien entendu, il ne viendrait à l'idée de personne de propager la syphilis ou la fièvre typhoïde, même si on avait la certitude de modifier dans un sens favorable l'évolution des générations futures, mais on pourrait certainement obtenir des résultats très intéressants en traitant l'homme et les animaux domestiques par certains antigènes et plus particulièrement. par certains microbes stérilisés, à doses stimulantes.

C'est là un problème d'une complexité très grande; 
son étude est hérissée de nombreuses diffieultés de toutes sortes. La plus grande de ces difficultés previent de ce que, ainsi que nous le verrons dans le ehapitre suivant (réactions provoquées par des agents psychiques), les effets stimulants se traduisent surtout par des manifestations d'ordre psychique ou moral qu'il est impossible de suivre avec quelque précision chez les animaux communs de laboratoires (1).

Sur l'homme, des essais dans cette direction ont été entrepris dans mon laboratoire, depuis sept ans (1913). Les hasards des expériences nous ont amené à injecter sous la peau des quantités très petites : $1 / 2$ à 2 millièmes de milligrammes de certains microbes non pathogènes (bac. coli, diplocoques, subtilis, etc.) tués par la chaleur, et nous avons obtenu ainsi des effets de bien-être tout à fait remarquables.

Très rapidement et souvent après une seule piqûre, les personnes de tout âge ne présentant aucun symptôme de maladie caractérisée, mais se sentant fatiguées, surmenées, courbaturées, sans appétit, sans sommeil régulier, éprouvaient brusquement un besoin d'activité cérébrale ou musculaire, qu'elles ne connaissaient plus depuis longtemps et pouvaient exécuter, avec entrain et sans aucune fatigue, des travaux ou des promenades qu'il leur était impossible de faire quelques jours auparavant.

On pouvait obtenir des effets analogues par beaucoup d'autres substances: lécithine, cacodylate de soude, phosphates sous différentes formes, diverses

(1) Peut-être pourrait-on obtenir quelques résultats intéressants chez les singes anthropomorphes ou chez certains perroquets très intelligents. 
préparations alcooliques, mais, dans tous ces cas, l'état d'euphorie est moins prononcé et surtout bien moins durable, que celui que l'on obtient par l'injection de certains microbes.

Dans ce dernier cas, l'état de bien-être général et de bonne humeur, provoqué par quelques piqûres ou ingestions de microbes, peut durer des mois et même des années.

Il est, bien entendu, difficile de prévoir quels seront les effets lointains de ces interventions, mais ce que nous avons pu en observer jusqu'à présent, ne nous fait présager aucune suite fâcheuse pour l'avenir.

En effet, les doses minimes de $1 / 2$ à 1 millième de milligramme par injection n'ont jamais provoqué de sensibilisation chez les personnes qui ont subi une série de 10 à 15 piqûres et dans les cas étudiés nous avons constaté, une régularisation et un retour à la normale des fonctions digestives, hépatiques vasculaires, rénales, respiratoires et 'surtout nerveuses, plus ou moins troublées. Il faut noter aussi que ces mêmes préparations microbiennes ne sont pas pathogènes pour les animaux de laboratoire, à des doses cent mille fois supérieures.

On est donc plutôt porté à admettre, qu'en faisant cesser ces troubles légers, on empêche le développement des affections plus graves, - qu'en un mot, les antigènes maniés avec des précautions nécessaires, peuvent être des agents très importants dans l'évolution progressive des individus et des espèces, surtout chez l'homme, où "le moral" tend de plus en plus à dominer "le physique ".

Le mécanisme de ces troubles, ainsi que de leur guérison par les antigènes, est difficile à suivre, ne 
serait-ce qu'à cause de la légèreté des symptômes que l'on a à combattre. On peut pourtant s'en faire une idée en prenant en considération le rôle du système nerveux dans l'économie générale, ainsi que la structure et le fonctionnement de ce système.

$\mathrm{Si}$, en effet, il nous faut admettre aujourd'hui, grâce aux travaux de la physiologie moderne, sur les sécrétions internes dont Brown-Séquard a été l'initiateur (1890), que certains produits des glandes à sécrétions internes peuvent agir directement sur l'économie générale ou sur certains organes, sans passer par les centres nerveux, l'idée exprimée par Claude Bernard :

“ Dans les organismes élevés, c'est seulement par l'intermédiaire du système nerveux qu'on agit sur la plupart des phénomènes vitaux ", doit toujours être considérée comme exacte, surtout quand il s'agit de l'organisme humain.

Le fait que la secrétine (substance produite par la partie de l'intestin qui touche à l'estomac, sous l'influence de l'acide chlorhydrique de l'estomac) excite la production du suc pancréatique par le pancréas, malgré l'interruption de toute communication nerveuse entre ces deux organes, ne prouve pas que la sécrétion de la pancréatine échappe à toute action nerveuse, et s'il en était ainsi pour le chien ou le lapin, rien ne prouve que les ćhoses se passent exactement de la même façon chez l'homme.

"Dans les organismes élevés ", a dit Claude Bernard, et nous avons vu, en parlant de l'évolution du système nerveux, que ce tissu a évolué beaucoup, depuis le chien jusqu'à l'homme civilisé. Nous pouvons en conclure qu'au volume et à la complexité des centres cérébraux, doit correspondre une plus 
grande finesse et sûreté de leurs connexions avec les glandes.

Quoi qu'il en soit, il est certain que même pour les animaux supérieurs, toutes les fonctions de l'organisme se trouvent sous le contrôle, sinon sous la domination complète, directe ou réflexe du système nerveux central.

Le phénomène de la respiration peut nous servir de type de ces réactions réflexes.

Les réactions d'oxydation et de réduction des cellules ont pour résultat la production d'un excès d'acide carbonique, qui passe dans le sang veineux et qui est éliminé ou "excrété" au dehors par le mécanisme suivant :

Parvenu dans les capillaires du poumon, l'acide carbonique passe du sang dans les cellules de la muqueuse pulmonaire dont il excite les terminaisons nerveuses, comme certains autres gaz excitent les cellules sensitives olfactives. Cette excitation est transmise au bulbe et passe de là aux muscles de la cage thoracique. Ces muscles se contractent, la cage thoracique se dilate et fait pénétrer ainsi dans le poumon l'oxygène de l'air qui, en neutralisant l'action excitante de l'acide carbonique, provoque, par retour, le relâchement des muscles.

La cage thoracique se rétrécit, une partie de l'oxygène inspiré passe dans le sang, tandis que l'air mélangé de l'excès d'acide carbonique est expulsé au dehors par la bouche.

L'asphyxie ou un trouble de la respiration peut donc être provoqué aussi bien par un arrêt dans le processus de réduction dans les cellules et par conséquent par manque d'acide carbonique dégagé, que par manque d'oxygène inspiré, et encore par 
un trouble dans les communications sensitivomotrices, entre la muqueuse pulmonaire et les muscles dis thorax, ainsi que dans les centres sensitifs et moteurs du bulbe et du cerveau.

Or, les travaux les plus récents sur la structure délicate des connexions nerveuses, inaugurés par J. Ramon Cajal (1), continués et complétés par Doomer, Van Gehuchten, Mann, Marinesco, Nageotte, etc., ont montré que les communications nerveuses sont assurées par des filets qui aboutissent, de place en place, à des ganglions composés de cellules nerveuses munies de ramifications très nombreuses appelées dendrites, hérissées elles-mêmes d'innombrables petites excroissances appelées épines.

Normalement, les épines de deux cellules nerveuses, placées vis-à-vis l'une de l'autre, ne se touchent pas, mais les contacts s'établissent aussitôt qu'une excitation périphérique ou centrale fait parvenir le courant nerveux à l'une de ces cellules ou à l'autre. Un tel ganglion peut donc être comparé à une station de commutateurs, dans lesquels les communications s'établiraient d'une façon automatique par le courant électrique mis en branle par la station centrale ou par un quelconque des postes périphériques.

Dans l'organisme vivant, le fonctionnement de ce système des commutateurs peut être troublé par toutes sortes de causes. Il a été constaté par l'étude histologique du cerveau et des ganglions des animaux expérimentalement surmenés par un travail forcé, chez les animaux plongés dans le sommeil hivernal,

(1) CAJAL, Histologie du système nerveux (variations normales et pathologiques du Neurone). Trad. française, Maloine, 1911. 
soumis à un excès de froid ou de la chaleur ou encore épuisés par la faim, que chez beaucoup de cellules, sinon chez toutes, les épines et les dendrites sont contractées et moins nombreuses, que beaucoup de contacts sont abolis, que les communications deviennent plus lentes, imparfaites ou sont rompues et qu'il en résulte des troubles dans le fonctionnement de certains organes.

Il est très probable que, chez l'homme, les mêmes causes peuvent produire des modifications de la même nature dans les neurones. Le surmenage, le froid, une alimentation insuffisante, des intoxications de toutes sortes, des émotions psychiques, peuvent brouiller ou arrêter les communications dans les stations de commutateurs, en déterminant la contraction d'un certain nombre de dendrites, diminuer les fonctions de certains organes, en affoler d'autres, amener, en un mot, des troubles fonctionnels qui se traduiront en dernier lieu par la fatigue, l'insommie, l'énervement, la perte de mémoire, etc.

C'est ainsi qu'il sera probablement possible d'expliquer l'action des hypnotiques, des anesthésiques, des alcools, etc., qui, à doses stimulantes accélèrent les passages des courants nerveux, les rendent plus sûrs, à doses plus fortes, les paralysent.

$\mathrm{Y}$ aurait-il, dans certains microbes, des substances qui agiraient mieux, d'une façon incomparablement plus active et plus durable, sur les cellules des ganglions nerveux, que les phosphates, l'arsenic, certains iodures, certains extraits d'organes, etc. ? Faudrait-il admettre que les mierobes de la flore intestinale normale jouent un rôle considérable dans le développement du tissu nerveux et par conséquent dans l'évolution progressive de l'homme et peut-être 
aussi dans celle des animaux ? Que la présence habituelle ou même constante de certaines espèces microbiennes dans l'intestin n'est pas le fait du hasard, mais résulte d'une adaptation réciproque qui a conduit à une symbiose en quelque sorte nécessaire ?

Autant de questions qu'il nous faut laisser provisoirement sans réponse. On peut affirmer toutefois que, si l'injection hypodermique d'une goutte de liquide contenant un demi-millième de milligramme de microbes (tués par la chaleur), dont certainement une petite fraction seulement représente la substance réellement active, peut produire chez l'homme une réaction très appréciable, les mêmes microbes vivants qui peuplent par milliards l'intestin, ne peuvent pas rester sans influence sur le fonctionnement général ou plus particulièrement sur certaines fonctions de l'organisme.

Ainsi posée, la question présente, croyons-nous, un intérêt considérable pour la biologie expérimentale.

3. Résumé. - En dehors de leur action par les éléments et les molécules qui les composent, action identique à celle provoquée par les éléments et les molécules libres d'origine quelconque, tous les colloïdes, quelle qu'en soit l'origine et la composition élémentaire, provoquent dans l'organisme pluricellulaire différencié une réaction, qui est toujours de même nature : la production des anticorps qui décomposent les colloïdes en molécules libres.

Suivant le rôle plus ou moins important pour l'organisme des organes qu'elle fait interyenir, et sui- 
vant son intensité, cette réaction peut stimuler les fonctions de certains organes et exercer une action favorable sur le développement de l'ensemble ou provoquer leur dégénérescence et arrêter le développement ou le rendre pathologique.

Cette réaction crée pour l'organisme un équilibre physico-chimique et fonctionnel nouveau, généralement très durable, et, par cette durée, elle modifie non seulement l'équilibre physiologique de l'individu, mais aussi de sa descendance.

L'introduction des colloïdes dans le milieu intérieur des organismes peut donc être considérée comme le facteur le plus important du développement progressif ou régressif des races, des mutations, transformations ou disparitions des espèces.

Un des plus importants sujets d'étude de la biologie expérimentale serait donc de classer les antigènes suivant leurs propriétés et leur action sur tel ou tel autre groupe d'arganes, de glandes ou de tissus, de façon à pouvoir les employer au développement des fonctions qu'on, aurait intérêt à perfectionner.

\section{9. - Le rôle des énergies radiantes dans l'évolution progressive}

Les énergies transmissibles, radiantes, que nous connaissons actuellement, sont: l'attraction universelle, l'électricité sous ses deux formes de courant et d'ondes électriques, les rayons ultra-violets, visibles ou lumineux, caloriques, les ondes sonores, et enfin les derniers venus, les rayons $\mathrm{X}$ ou $\gamma$ et les rayons $\beta$.

Nous avons vu déjà, dans les chapitres consacrés au son et à l'évolution du tissu nerveux, quelle est 
la nature de l'action de la lumière et du son sur le développement des êtres vivants. Nous ne connaissons encore rien du mécanisme intime de cette action, pas plus que de celle des substances odorantes ou pourvues de saveurs particulières sur la formation des cellules olfactives ou des papilles gustatives. Nous savons seulement que, s'il n'y avait pas de lumière se propageant dans l'espace par des ondes d'un certain rythme, les animaux seraient certainement dépourvus d'organes de vision et de centres visuels correspondants et qu'il en serait de même pour tous les autres organes des sens périphériques et de leurs centres dans l'encéphale, qu'il y a donc une corrélation étroite de cause à effet entre l'existence des uns et la formation des autres.

Peut-on asigner un rôle spécial à l'attraction universelle et à l'électricité dans l'évolution progressive de la matière vivante? C'est peu probable. Il est possible que c'est sous l'action de la pesanteur que se sont formés les canaux semi-cireulaires dans lesquels on place les sens de l'espace et de l'orientation. Il est probable que l'organe électrique du gymnote électrique, de la torpille et de presque toutes les raies a pour origine des courants électriques, mais on ne trouve nulle part des témoignages anatomiques d'une influence quelconque de ces énergies sur le développement de l'encéphale et par là sur l'évolution progressive des animaux. On peut dire que, comme facteurs primordiaux de l'évolution, ces formes d'énergie n'influencent pas autrement la matière vivante que la matière inorganique.

Le rôle de l'énergie calorique dans l'évolution des animaux apparaît comme au moins aussi considérable que celui de la lumière et du son. La rapidité 
avec laquelle se succèdent les formations de plus en plus parfaites, à partir du moment où on constate l'apparition d'une température de plus en plus constante dans le milieu intérieur de l'organisme (oiseaux et mammifères), serait déjà un indice assez clair de l'importance de ce facteur, mais on sait aussi quelle influence considérable les moindres changements de température exercent sur les réactions chimiques qui sont à l'origine de toute fonction organique.

Il y a bien des protozoaires qui s'accommodent d'une température presque glaciale. On a même remarqué que dans les mers polaires les noctiluques, ainsi que d'autres infusoires, sont plus nombreux que sous l'équateur; mais il est certain, que les animaux pluricellulaires ne peuvent vivre activement à une température inférieure à $10-15^{\circ}$, parce qu'à cette température l'action de beaucoup de diastases se ralentit ou s'arrête même complètement et que les conditions " optima » d'une vie active sont réalisées à une température moyenne de 15 à $25^{\circ}$.

Les vers, les insectes, les mollusques, les amphibiens, les reptiles des régions tempérées passent la moitié de leur vie dans un état de léthargie voisin de la mort et perdent ainsi beaucoup de temps que les animaux à sang chaud, c'est-à-dire à température constante (de 36 à $40^{\circ}$ ) mettent à profit pour la progression de leur développement individuel.

De plus, il est à remarquer que la continuité et la régularité d'un travail est une condition au moins aussi importante du perfectionnement que son intensité.

On sait aussi que la faculté de produire des anticorps est bien moins développée chez les animaux à sang froid (ou plutôt à température ambiante). 
Ainsi, dans ses intéressantes recherches sur la toxine tétanique, Metchnikoff a observé que les crocodiles supportaient très bien, sans en être malades, des quantités énormes de cette toxine et qu'ils produisaient de l'antitoxine beaucoup plus rapidement que les chevaux, à la condition toutefois d'être placés dans une eau maintenue constamment à une température supérieure à $30^{\circ}$, condition qui n'est jamais réalisée dans la nature, même dans les rivières de l'équateur. Quand on maintenait les caïmans à une température voisine de $20^{\circ}$, la toxine injectée disparaissait au bout d'un certain temps, elle était détruite, mais ne provoquait pas la formation de l'antitoxine.

Cantacuzène, Metalnikoff et d'autres biologistes ont réussi à immuniser des insectes contre certaines maladies expérimentales, mais toujours en maintenant ces animaux à l'étuve, à une température de 35 à $37^{\circ}$. On ne sait pas si les invertébrés vivant dans des conditions naturelles peuvent s'immuniser contre certaines maladies infectieuses spontanées. Dans nos expériences sur les infections des insectes par les champignons pathogènes, dans des conditions aussi naturelles que possible, nous n'avons jamais rencontré, sur des centaines de mille contaminés, un seul individu réfractaire.

Or, nous avons vu combien l'état d'immunité-anaphylaxie acquise peut être important pour l'évolution de l'individu et de l'espèce et nous croyons, que ce facteur de l'évolution n'a pu entrer en jeu qu'avec la formation d'un milieu intérieur pourvu d'une température constante d'environ $36^{\circ}$, qui a coïncidé avec un certain degré de développement de l'encéphale. 


\section{* *}

On ne sait pas si les rayons de Rœentgen sont produits spontanément dans la nature, mais on doit admettre qu'une certaine quantité, il est vrai minime, des rayons $\gamma$ s'échappent continuellement de la croûte terrestre. On sait que les différents tissus sont très différemment sensibles à l'action de ces rayons, mais on ne connaît encore aucun organe différencié qui pourrait les recevoir et aucun centre pour les enregistrer.

Il en est de même pour les rayons $\beta$, pour lesquels les organismes vivants ne possèdent aucun organe spécialement sensible, mais d'après les recherches de H. Zwaardemaker, le potassium, dont le corps humain normal possède 40 grammes, c'est-à-dire une quantité suffisante pour ioniser l'air, agit surtout par ses propriétés radioactives.

D'après Campbell et Wood, 40 gr. de potassium dégageraient autant de rayons $\beta$ très pénétrants, qu'un deux millièmes de milligr. de radium pur et fournirait ainsi assez de rayons $\beta$ pour exercer une action physiologique manifeste sur l'organisme.

Zwaardemaker démontre, en effet, que certaines contractions musculaires ne peuvent être obtenues qu'en présence d'une certaine quantité de potassium et que cette action est due exclusivement à la radioactivité, parce que le potassium peut être remplacé dans son action physiologique par toute autre substance radioactive dégageant la même quantité des rayons $\beta$ et ne peut être remplacé par aucune substance non radioactive, mais possédant des propriétés chimiques analogues.

Pour nous rendre compte si le potassium aurait DANysz. - La genèse de l'énergie psychique. 
pu jouer un rôle notable dans l'évolution des êtres vivants, il serait nécessaire d'en évaluer la quantité proportionnelle chez les animaux de différentes classes, mais comme on sait qu'il y a relativement beaucoup plus de potasse dans les tissus des végétaux que chez l'homme, il serait difficile de chercher dans la radioactivité un agent de l'évolution progressive.

En résumé, ce qu'il importe de noter, c'est que de toutes les énergies rayonnantes, seules, la lumière le son et la chaleur ont exercé une influence décisive sur l'évolution progressive. Les deux premières comme formatrices de deux principaux organes des sens et par là de deux importants centres nerveux, la chaleur, comme stimulant et régulateur des réactions chimiques, qui sont à la base de toute fonction physiologique de l'organisme.

Et il nous faut ajouter que de même que les substances (cristalloïdes ou colloïdes), les énergies rayonnantes n'ont pu favoriser l'évolution qu'à doses st mulantes, c'est-à-dire modérées. Une lumière trop intense, un bruit trop violent sont aussi nocifs pour l'organisme que les poisons les plus toxiques et on sait qu'une température ambiante trop élevée, voisine de $30^{\circ}$, rend tout effort volontaire physique ou intellectuel plus pénible qu'une température de $20^{\circ}$, de sorte que chez les êtres supérieurs elle ralentit l'évolution plutôt qu'elle ne la favorise.

\section{0. - Le rôle des énergies nerveuses dans l'évolution}

1. Réactions chimiques provoquées par les émotions. - Dans les chapitres qui précèdent, nous avons 
eu à considérer les réactions provoquées dans l'organisme par les substances sous forme d'éléments simples, de molécules plus ou moins complexes et de micelles, et nous avons vu que l'action des énergies physiques peut être assimilée à celle des substances étrangères, parce que les modifications chimiques provoquées par ces énergies dans la matière vivante sont les mêmes, ou de la même nature que dans la matière inerte.

Il en est tout autrement des énergies que l'on pourrait appeler métaphysiques, parce qu'elles n'exercent aucune action appréciable sur la matière inerte et même, peut-on dire, sur la matière vivante, dans le sens général de ce mot.

Les émotions, la peur, la joie, le chagrin, la colère, la pitié, l'amour, etc., ne peuvent être ni mesurés, ni transmis comme la lumière, la chaleur ou l'électricité; ce sont là pourtant bien des énergies, non seulement parce que ce sont les leviers les plus puissants des réactions sociales, mais aussi parce qu'elles provoquent dans les cellules des organismes sensibles des réactions chimiques identiques à celles que l'on peut provoquer par des substances ou par des énergies physiques.

Nous ne connaissons encore que les effets apparents, superficiels, de ces réactions; nous ignorons complètement leur mécanisme chimico-physique intime; mais nous pouvons affirmer avec certitude qu'aucune manifestation vitale, qu'elle soit purement mécanique, comme une contraction des pseudopodes d'une amibe, ou hautement psychique, comme la pensée ou l'acte réfléchi d'un savant oud'un artiste, ne peut avoir d'autre origine qu'une réaction chimique, ne peut être exécutée que par 
une série de ces réactions, qui se déterminent et se commandent les unes les autres.

Les observations et les recherches expérimentales des biologistes et des physiologistes modernes: J. Loeb, Pavlow, N.-B. Cannon, Dumas, Kendall, etc., dont quelques-unes sont résumées dans l'ouvrage de G. Bohn et A. Drzewina: La chimie de la vie, le prouvent d'une façon incontestable.

Ainsi Kendall a observé que, chez un chat qui a subi une forte émotion désagréable, les capsules surrénales produisent une substance, "la préurée ", qui transforme le carbonate d'ammoniaque en urée, substance qui ne se forme pas à l'état normal: N.-B. Cannon et D. de la Paz ont montré, par une série d'expériences sur des animaux, que les émotions ont pour effet d'augmenter notablement la sécrétion d'adrénaline, ce qui expliquerait la glycosurie d'origine émotive. Ce sont là des preuves indiscutables de ce fait, que des réactions chimiques peuvent être provoquées par des réactions psychiques, parce que ces émotions aboutissent sans intervention d'aucune substance étrangère à la formation de composés chimiques simples, faciles à caractériser et à doser.

Chez l'homme qui peut s'observer lui-même, noter les réactions qu'il éprouve et en analyser le mécanisme apparent, on constate une délicatesse de ces réactions et de la différenciation des organes dont ces réactions sont le siège, dont il est encore difficile de se faire une idée exacte.

La vue d'un objet ou d'une personne aimée ou haïe est fixée dans la rétine par une réaction de la même nature; elle est transmise aux centres visuels du cerveau de la même façon, mais là commencent les différences: l'image de la personne aimée et 
celle de la personne haïe sera très probablement transmise à des compartiments différents des sphères d'association, ou bien si les deux images touchent les mêmes centres, leur révélation dans la conscience provoquera des réactions chimiques très différentes, d'abord dans les centres de l'écorce cérébrale, ensuite dans les systèmes glandulaires et, par retour, de nouveau dans le système nerveux central, où il éveillera des réflexes et des manifestations volontaires différentes, par conséquent des réactions chimiques encore différentes.

Une impression visuelle ou, plus généralement sensorielle agréable, touchera, suivant sa nature, l'hypophyse, la thyroïde ou la glande intersticielle ou les glandes surrénales, une impression désagréable sera transmise plutôt au foie. Les deux impressions provoqueront donc des réactions différentes dans les centres cérébraux; ces réactions seront transmises par des fibres nerveuses différentes à des organes différents, et comme elles auront pour résultat définitif la formation des composés chimiques très différents, il faut bien admettre que les différentes émotions n'ont pas pour origine des réactions chimiques identiques.

Il y a donc là quelque chose de particulier qui mérite une étude approfondie, un phénomène nouveau qui a suscité autant de discussions pasionnées que l'apparition de la vie, le passage de la matière inerte à la matière vivante, ici l'apparition de l'intelligence ou le passage de la sensibilité végétative ou, si l'on veut, purement chimique, à la sensibilité consciente qui est à l'origine de toutes les propriétés ou qualités de l'ame.

Nous venons de le dire: les énergies métaphysi-. 
ques n'exercent aucune action appréciable sur la matière inerte, et même sur la matière vivante, dans le sens général de ce mot.

En effet, un être unicellulaire, un végétal, et même une cellule vivante isolée d'un organisme supérieur, un leucocyte, une cellule conjonctive ou une fibre musculaire de l'homme que l'on peut faire vivre en dehors de l'organisme (Carrel), ne réagiront nullement, bien que vivantes, à la peur ou à la joie. Ces énergies ne peuvent agir que sur les cellules faisant partie de l'organisme des êtres pourvus d'un système nerveux différencié et reliés à l'ensemble par ce système nerveux. Elles agiront sur ces êtres en raison du degré de développement et de différenciation du tissu nerveux. Mais alors, puisque chez les êtres qui en sont conscients, ces énergies provoquent des réactions chimiques dans les cellules qui, elles-mêmes, n'en ont pas conscience, elles agissent en dernier lieu sur la matière inerte, sur les réactions des éléments inertes en soi, dont les cellules sont formées, elles ne sont donc pas métaphysiques. Ce sont simplement des énergies nerveuses ou une forme d'énergie nouvelle qui n'apparaît qu'à un certain stade de l'évolution synthétique de la matière vivante, comme l'affinité chimique qui n'existe pas dans les électrons, apparaît sous forme d'une énergie nouvelle à la suite et comme conséquence de la condensation des électrons en atomes et en molécules élémentaires, comme le phénomène vie résulte de la synthèse des molécules en micelles albuminoïdes.

Est-il possible de tracer une limite entre la vie consciente et inconsciente, de désigner avec certitude l'espèce animale chez laquelle on pourrait déceler les premières manifestations rudimentaires d'une 
sensibilité consciente? Ce sont là encore des sujets d'études, que la biologie expérimentale n'a pas abordé avec méthode et les belles expériences de Fabre sur la vie des insectes montrent bien que l'interprétation des faits observés sera souvent hérissée de difficultés presque insurmontables.

Chez beaucoup d'insectes les actes qui, à première vue, semblent volontaires, sont en réalité accomplis aujourd'hui d'une façon complètement inconsciente, pour ainsi dire automatique, et il sera souvent bien difficile d'expliquer comment et pourquoi un acte dont l'accomplissement parfait semble avoir demandé une longue série d'expériences, c'est-à-dire de manifestations volontaires, a pu devenir inconscient (1).

Quoi qu'il en soit, ce que l'on peut affirmer avec certitude, c'est l'évolution parallèle du cerveau et de la sensibilité consciente, et, quand on arrive à l'homme, la prédominance de plus en plus marquée, d'une part, du système nerveux sur toutes les réactions inconscientes de l'organisme, d'autre part, des émotions conscientes sur toutes ces réactions involontaires.

On peut affirmer aujourd'hui avec certitude que si un indigène du centre de l'Afrique ou de la Poly-

(1) La guepe des murailles (amophyle), par exemple, construit ses nids sur des murs rugueux. Elle construit d'abord une cellule dans laquelle elle place une ou plusieurs araignées tuées, ensuite elle pond un œuf dont la larve aura ainsi une nourriture préparée jusqu'à sa transformation en nymphe. Ceci fait, la guêpe ferme la cellule et recouvre le tout d'un enduit en tout semblable aux rugosités du mur. C'est done là un travail très complexe. Or, quand on enlève les araignées aéposées au fond de la cellule, l'insecte pond son œuf et continue tout le travail comme si rien d'anormal ne s'était passé. On peut même enlever l'œuf et la cellule, l'insecte ne reconstruira pas la cellule, mais il mettra l'enduit comme si tout était en place. 
nésie vit encore d'une façon à peine plus consciente qu'un mammifère supérieur, cheż l'homme civilisé et cultivé, la sensibilité consciente, l'état moral, do-, mine de plus en plus complètement le fonctionnement des organes, c'est-à-dire, en dernière analyse, les réactions chimiques et les fonctions physicochimiques des cellules.

Ainsi, tout en reconnaissant, que si la sensibilité consciente qui est à l'origine de toutes les manifestations de l'intelligence, peut et doit être l'objet d'une étude abstraite et comme telle métaphysique, elle doit être avant tout étudiée au point de vue biologique, parce que, pour l'homme, la sensibilité consciente est devenue le levier le plus puissant de l'évolution progressive individuelle et sociale. Chez l'homme cultivé, ce sont les événements de la vie quotidienne, les impressions bonnes on mauvaises, agréables et désagréables, qui règlent bien plus les sécrétions de ses glandes, le fonctionnement de ses organes, les conditions de nutrition de l'organisme et, par conséquent, l'état de santé générale, que la nature des aliments qu'il absorbe. On peut donc soutenir aujourd'hui que, chez l'homme civilisé, l'équilibre ou l'état de santé morale aura autant d'influence sur l'évolution de l'individu et de la race que son état d'équilibre physique.

Ce n'est pas là, bien entendu, la seule condition que nous ayons à considérer dans l'évolution de l'humanité, mais c'est certainement la plus importante. Les maladies infectieuses, en provoquant un certain nombre de décès prématurés, agissent plutôt sur la quantité que sur la qualité. Certaines d'entre elles, les maladies vénériennes en particulier, affectent temporairement l'état moral des individus; mais ce sont 
là des accidents plus rares que les émotions qui peuvent survenir à tout instant et sont souvent, à l'origine, les causes premières des affections chroniques et même de certaines maladies infectieuses.

Nous avons vu, en effet, que les membranes muqueuses normales sont imperméables pour les colloïdes, les albumines et les microbes, en un mot pour les antigènes, mais qu'elles laisseront passer toutes ces substances, quand elles seront congestionnées. On sait aussi que les émotions provoquent souvent ces congestions, surtout dans les muqueuses intestinales, et alors elles laissent passer dans le sang, soit des substances albuminoïdes plus ou moins incomplètement digérées qui, agissant comme antigènes, provoquent des réactions pathologiques qui se traduisent par toutes sortes d'affections chroniques, soi des microbes vivants qui, dans certains cas, peuvent devenir infectieux.

Les émotions qui nous viennent du milieu extérieur, de même que celles qui ont pour origine le fonctionnement anormal d'un organe du milieu intérieur (douleurs de toutes sortes), provoquent des réactions réflexes, et tout animal pourvu d'un système nerveux différencié et de centres nerveux capables de les ressentir et d'en avoir conscience, en est tributaire. Beaucoup d'animaux domestiques, et probablement aussi de ceux qui vivent à l'état sauvage, ressentent des joies et des chagrins qui peuvent influencer en bien ou en mal le métabolisme intime de leurs organes et avoir des répercussions favorables ou nocives pour le développement ultérieur de l'individu et de sa descendance. 
Chez l'homme, comme chez les animaux, seuls les muscles striés et les appareils munis de ces muscles: les membres, la cage thoracique, l'abdomen, les sphincters, etc., obéissent directement à la volonté et dans ces cas aussi, l'ordre donné par la volonté aux centres moteurs, ainsi que les contractions ou les relâchements musculaires, ont pour point de départ des réactions chimiques. Et si la volonté n'a aucune action appréciable ni sur les fibres musculaires lisses dont sont formées les parois du cœur, des vaisseaux sanguins et du tube digestif, ni sur tous les autres tissus qui composent l'organisme, c'est que la volonté ne sait pas encore provoquer les réactions chimiques nécessaires dans les centres cérébraux, ou qu'il n'existe pas encore de liaisons nerveuses directes ou indirectes entre les centres moteurs et les organes ou tissus qu'il faudrait actionner.

Toutefois, chez l'homme, la puissance de la volonté peut être augmentée par des exercices et un entraînement appropriés. Il y a déjà des hommes plus ou moins capables de maîtriser les émotions qu'ils éprouvent et par conséquent aussi les réactions réflexes qui en découlent, et il n'est pas impossible de prévoir (voir chap. 28) la formation dans le pallium de centres nouveaux et de connexions nouvelles, qui permettront à la volonté d'influencer directement les réactions du milieu intérieur.

2. Intervention des conceptions, conscientes. Jusqu'à nouvel ordre, l'intelligence ne peut donc agir sur le développement de l'organisme, sur les fonctions et, par conséquent, sur la formation des organes, que d'une façon indirecte, en cherchant, par une expérimentation appropriée, des substances 
qui, par leurs propriétés chimiques, provoquent certaines réactions physiologiques. En appliquant ces substances dans les cas où il s'agit de stimuler les fonctions normales d'un organe défaillant, on peut rétablir ainsi l'équilibre fonctionnel général momentanément rompu.

Cette période de l'intervention consciente du cerveau dans sa propre évolution ne fait que commencer. Elle doit s'appuyer sur les sciences expérimentales et spéculatives, qui forment l'ensemble de notre savoir et on peut dire, qu'en dernière analyse, tous ces efforts ne peuvent avoir d'autre raison d'être, d'autre but, que l'évolution toujours progressive de ce cerveau, de l'intelligence, qui, une fois éveillée, ne peut trouver nulle part ailleurs de satisfaction durable, que dans.la recherche des moyens de son propre perfectionnement à l'infini.

Cette recherche n'est pas encore organisée, comme nous prévoyons qu'elle pourrait l'être. Les découvertes sont dues plus souvent à des hasards heureux qu'à des plans de travaux méthodiques, à des révélations mentales involontaires plutôt qu'à des idées préconçues, mais il n'en est pas moins certain que les trouvailles du hasard ne sont possibles qu'au cours des recherches, que les révélations ne surgissent que dans les cerveaux en travail, et enfin que tout effort cérébral, quel qu'en soit le résultat immédiat, concourt au développement et à la différenciation de plus en plus délicate, anatomique, histologique et chimique de l'organe de la pensée.

Un travail cérébral encore confus et inexprimé du père se cristallisera souvent en activité coordonnée d'une puissance et d'une richesse remarquables chez le fils ou le petit fils. 
Ce que nous savons aujourd'hui des interventions conscientes possibles dans l'évolution de l'énergie psychique est encore bien peu de chose en comparaison de ce que nous prévoyons qu'on pourrait ou devrait savoir, non pas encore pour augmenter directement et sûrement sa puissance, mais simplement pour combiner un programme de recherches, qui pourraient nous conduire à une connaissance plus exacte des effets immédiats ou lointains provoqués par ces interventions.

Toutefois, les études concernant la composition et la constitution chimique des substances que nous savons préparer dans nos laboratoires (alcaloïdes, acides-aminés, vitamines, etc.) et qui, suivant les doses employées, peuvent provoquer des réactions stimulantes ou nocives sur tels ou tels autres centres nerveux, d'une part, le rôle physiologique des organes du milieu intérieur de l'organişme (hypophyse, système thyroïdien, surrénales, glande intersticielle, etc.), ainsi que la nature chimique de leurs sécrétions d'autre part, et enfin sur l'importance de l'éducation méthodique des organes des sens, se multiplient chaque jour.

Les résultats de ces recherches, encore souvent difficiles à apprécier à leur juste valeur et à coordonner, sont pourtant aujourd'hui soigneusement enregistrés dans des recueils spéciaux et serviront en temps utile à l'établissement des lois biologiques générales, qui permettront de mettre chaque fait à sa place et de donner à chaque phénomène une explication assez claire pour servir de base solide à des recherches de plus en plus délicates et précises. 
Ainsi les résultats infaillibles et merveilleux obtenus dans le traitement du myxœdème et des états crétinoïdes par l'extrait des glandes thyroïdes, ont montré que le développement du cerveau ainsi que la formation du squelette, sont réglées par les sécrétions de ces glandes. On sait aussi que les sécrétions de l'hypophyse modèrent le développement des os longs, que le développement et le fonctionnement normal des glandes génitales mâles contribue à l'ossification normale des cartilages et au développement du cerveau et vice versa, que la destruction d'une partie de l'écorce cérébrale, qui ne met pas la vie de l'animal en danger (l'expérience a été faite sur les gallinacées), amène une strophie notable des glandes sexuelles non seulement chez les individus traités, mais aussi chez leur descendance.

Ces expériences, bien qu'encore grossières, montrent donc d'une façon indiscutable, tout d'abord, qu'il y a des corrélations très étroites entre les fonctions, et par conséquent le développement de différentes glandes et du cerveau ensuite, qu'une connaissance plus approfondie de la nature chimique des sécrétions internes et de leur rôle nous permettra d'intervenir sciemment et utilement dans le développement de l'intelligence, non seulement en agissant directement sur le cerveau, mais aussi indirectement par l'intermédiaire de ces glandes.

3. En résumé, on peut done dire que les émotions sont des énergies, qui sous leurs formes les plus simples, commencent à s'élaborer chez les animaux invertébrés et vertébrés pourvus d'un cerveau et dont la formation peut être provoquée par des sensations, 
des phénomènes ou des événements qui peuvent être quelconques et qui frappent l'imagination non pas tant par leur nature que par l'importance des effets réels ou imaginés, agréables ou désagréables, qu'ils peuvent avoir pour l'organisme.

Pour qu'il y ait "émotion", il faut donc un certain degré de conscience et d'imagination, c'est-à-dire des centres cérébraux d'un certain développement.

Il y a certainement des animaux, les cœlentérés, les échinodermes, les vers inférieurs, incapables d'une émotion, à quelque degré que ce soit, mais il serait difficile de désigner l'espèce qui, la première, a pu ressentir une émotion quelconque. Ce qui n'est pas douteux, c'est qu'à partir de ce moment, à la suite des impressions sensorielles, les émotions ont joué un rôle considérable dans le développement de la conscience et par conséquent des centres nerveux, autant par les réactions qu'elles ont provoquées directement dans le cerveau, que par leurs actions réflexes sur les organes et, par retour, par les organes (glandes thyroïdes, etc.), sur le cerveau.

Chez l'homme, le rôle de ces énergies, dont une certaine catégorie sont devenues des sentiments, est devenu dominant dans le développement simultané ou plutôt progressivement alternatif du cerveau et de l'intelligence et par là de la volonté qui, chez l'homme cultivé, commence à maîtriser les émotions et devient ainsi actuellement le facteur le plus important de l'évolution progressive.

\section{1. - Le rôle des acides aminés et des vitamines}

Acides aminés, vitamines. - L'étude du rôle des différents facteurs du milieu extérieur et du milieu 
intérieur dans l'évolution de la matière vivante serait incomplète si nous n'y ajoutions un résumé de nos connaissances sur l'action particulière de certaines substances azotées: des acides aminés et des vitamines, sur la nutrition générale des animaux ainsi que sur le développement de certains organes.

On savait depuis longtemps que les plantes peuvent se nourrir de matières purement minérales, tandis que les animaux ne peuvent exister qu'à la condition d'avoir à leur disposition des substances organiques, des albumines préparées par les végétaux.

L'étude plus précise de ces questions ne date pas de plus d'une trentaine d'années. Ainsi Winogradsky a montré en 1890 (Annales de l'Institut Pasteur) que les nitromonades, microbes qui transforment les sels ammoniacaux en nitrates, peuvent vivre et se multiplier dans un milieu composé exclusivement de substances minérales d'origine inorganique et notamment de sulfate d'ammoniaque, de phosphate de potassium, de carbonate de magnésie ou de calcium et d'eau distillée. Mazé a constaté, par une série d'expériences précises, dont les dernières datent de 1919. qu'un végétal supérieur, le maïs, peut vivre et accomplir toute son évolution dans un milieu composé de quinze éléments simples, à l'exclusion de toute matière organique.

On ne sait pas, si des animaux tout à fait inférieurs, tels que les porifères (éponges) ou des cœlentérés (méduses) pourraient vivre dans un milieu exclusivement minéral, mais on sait avec certitude que les oiseaux et les mammifères meurent d'ination ou ne se développent pas, quand on les prive de certains acides-aminés, qu'ils succombent à des maladies spéciales: béri-béri, scorbut-polynévrites, 
quand on les nourrit avec des albumines et des graisses d'origine animale ou végétale, mais privées de vitamines.

Nous avons vu plus haut que les acides-aminés sont des substances cristalloïdes, composées de carbone, d'hydrogène, d'oxygène et d'azote, que l'on obtient par l'hydrolyse ou la digestion des micelles albuminoïdes.

On en 'connaît une quinzaine aujourd'hui et on sait que ce sont ces substances qui seules peuvent être absorbées par les cellules qui composent l'organisme animal et servir au processus de respiration, de construction ou d'entretien des micelles albuminoïdes qui composent les cellules.

Or, il résulte des expériences inaugurées au début de ce siècle et poursuivies surtout par des biologistes américains (Hopkins et Wilcock, Osborne et Mendel, Ackroyd, etc.), que si certaines de ces substances, par exemple, la tyrosine, peuvent être combinées par l'organisme aux dépens de substances plus simples, d'autres, comme le tryptophane, la lysine, l'histidine, la cystine, doivent être absorbées telles quelles et ne peuvent pas manquer dans le bol alimentaire, sous peine d'exposer l'animal à des désordres graves.

Ainsi Hopkins et Wilcock ont nourri des souris avec de la zéine, une albumine retirée du maïs, qui contient 16,13 p. 100 d'azote, mais dont l'hydrolyse ne fournit pas de tyrosine, de lysine ni de tryptophane et des quantités relativement très petites d'arginine et d'histidine, et ont constaté un amaigrissement considérable chez les adultes, un arrêt de développement chez les jeunes. L'addition de la tyrosine à la zéine ne modifie en rien cet état de 
choses, tandis que l'addition de tryptophane améliore très nettement l'état physiologique des jeunes et des adultes, sans pourtant influencer la croissance des jeunes. Osborne et Mendel ont montré que, pour faire pousser les jeunes souris ou rats jusqu'à une taille normale et les faire se reproduire, la lysine est indispensable. Il en est de même de la cystine, de l'histidine et de l'arginine dont l'absence arrête le développement des animaux et dont l'addition le fait reprendre.

Le rôle des vitamines semble être tout autre. Les premières recherches concernant ces substances, dont on ne connaît pas encore exactement la composition chìmique, sont à peu près contemporaines de celles concernant le rôle de certains acides aminés. Leur point de départ est l'observation d'une maladie, appelée béri-béri, assez fréquente en Extrême-Orient. chez des marins qu'on nourrissait presque exclusivement avec du riz décortiqué, tandis que ceux qui mangeaient du riz non décortiqué ne devenaient pas malades. Grings d'abord, et puis Eykmann, ont eu l'idée que ces polynévrites pouvaient avoir pour origine cette alimentation exclusive et ont constaté, qu'en effet, les pigeons nourris exclusivement avec du riz bien poli, présentaient, au bout d'un certain temps, les mêmes symptômes pathologiques et qu'il suffisait de leur donner un peu de son de riz pour les en préserver ou les guérir.

Mais c'est à Stepp que l'on doit d'avoir réuni toutes les données de ce problème par une expérience aussi simple que précise. Il a montré: $1^{\circ}$ que les souris nourries exclusivement avec du pain ordi-

DAnysz. - La genèse de l'énergie psychique. 
naire vivent et se multiplient d'une façon normale; $2^{\circ}$ que les souris nourries avec le même pain soigneusement lavé par l'alcool succombent rapidement à la cachexie; $3^{\circ}$ que le pain lavé par l'alcool et additionné de substances extraites par l'alcool et desséchées par évaporation, redevient aussi nourrissant que du pain frais ordinaire, tandis que le pain lavé, additionné de graisses neutres, de la lécithine, de la cholestérine, des cérébrosides que l'on pouvait supposer enlevés par l'alcool, se montrait un aliment aussi peu suffisant que le pain lavé tout seul.

Il était donc démontré que dans le pain, de même que dans l'enveloppe des grains de riz, il y a une substance absolument indispensable à l'assimilation des matières alimentaires contenues dans les grains de riz et dans le pain, par l'organisme des souris et des pigeons, et c'est à cette substance qu'il a pu obtenir à l'état cristallisé que Casimir Funk, chimiste polonais attaché à un laboratoire de médecine expérimentale de Londres, a donné le nom de Vitamine.

L'importance de cette découverte a, bien entendu, suscité de nombreux travaux dans les laboratoires de tous les pays et on a reconnu rapidement que toutes les substances alimentaires animales et végétales contiennent leurs vitamines, qu'il y a plusieurs sortes de vitamines qui diffèrent entre elles par quelques caractères chimiques et physiques, et que les substances alimentaires ne peuvent être digérées et assimilées que quand elles sont absorbées avec ces vitamines. On a reconnu aussi que ces dernières sont détruites par un chauffage prolongé, et enfin qu'une vitamine peut être souvent remplacée par une autre. Ainsi le riz décortiqué, indigeste et inassimilable, 
peut devenir un élément complet et sain quand on l'additionne d'une certaine quantité, même très petite, de levure de bière.

Nous n'avons pas à entrer ici dans les détails de la composition chimique des vitamines. Celle isolée par Funk des balles de riz se rapprocherait, par sa composition du groupe des purines, d'autres sont moins bien connues. Les unes sont solubles dans l'eau et dans l'alcool, d'autres dans les graisses. Les unes sont détruites par une chaleur modérée $\left(55^{\circ}\right.$ à $\left.60^{\circ}\right)$, d'autres résistent à l'ébullition prolongée. La chimie ne tardera certainement pas à établir avec précision toutes ces données.

Nous ne sommes pas beaucoup plus avancés dans la connaissance du mécanisme de l'action physiologique des vitamines.

On sait que tous les tissus actifs des animaux: les muscles, les glandes, le lait, les œufs, contiennent toujours une certaine quantité de vitamines: les expériences de Mc. Collum, Simmonds et Pitz, de Hart, etc., semblent prouver que l'organisme des animaux ou du moins des oiseaux et des mammifères est incapable de les produire, que la quantité de vitamines contenue dans les tissus n'est qu'une provision qui s'épuise toujours assez rapidement, en quelques jours, tout au plus en quelques semaines, si on ne la renouvelle pas constamment.

Une femelle de rat pourvue d'une ration alimentaire complète et abondante, mais privée de vitamines, ne parvient pas à élever ses petits. Leur croissance s'arrête rapidement et ils ne tardent pas à 
périr. On a constaté chez une vache traitée de la même façon, que ce fait n'est pas dû à une diminution de la quantité de lait fourni par la vache et absorbé par le veau, mais autant qu'on peut en juger, uniquement à l'absence des vitamines, dont la réserve contenue dans le corps de la mère a été épuisée.

Les expériences récentes de A. Lumière sur les pigeons nourris avec du riz poli tendent à prouver que le rôle des vitamines apportées avec les aliments consiste surtout dans l'excitation des glandes, dont les sécrétions opèrent la digestion des aliments ingérés (salive, suc gastrique, bile, suc pancréatique, etc.) et qui, sans le concours des vitamines, deviennent insuffisantes.

$\mathrm{Ou}$ bien les vitamines jouent-elles le rôle d'un complément nécessaire à toute réaction de digestion ou d'hydrolyse?

"Nous avons montré, dit Lumière, que les pigeons alimentés exclusivement avec du riz poli perdent rapidement l'appétit et que la plupart d'entre eux meurent avec tous les symptômes de l'inanition. Il nous a paru intéressant de chercher la cause de cette anorexie... La sensation de la faim semble correspondre à des besoins cellulaires, elle cesse, non quand ils sont satisfaits, mais dès que l'estomac renferme les aliments nécessaires à cette satisfaction. Il suffit dès lors que le contenu stomacal ne puisse s'évacuer pour empêcher le retour de l'appétit. "

Ce raisonnement n'est peut-être pas tout à fait juste, parce qu'on peut éprouver un manque d'appétit avec un estomac complètement vide. Il y a des émotions qui "coupent l'appétit", mais les faits constatés par Lumière n'en sont pas moins intéressants. Le riz poli mangé par le pigeon avec plaisir 
le premier jour de l'expérience, reste dans son estomac, où il forme une masse sèche et compacte, tandis que la même quantité de riz non décortiqué est broyée, digérée et évacuée en quelques heures. Le premier pigeon refuse de manger du riz poli les jours suivants, et si on le gave, on ne fait que remplir l'estomac sans aucun profit pour l'animal; au contraire, plus la nourriture avitaminée est abondante, plus la déchéance et la mort de l'animal ainsi traité est rapide.

Il suffit alors de quelques centigrammes d'un extrait de levure ou de balle de riz pour faire sourdre les ferments digestifs et ramener les animaux en expérience à l'état normal.

Le rôle des vitamines comme excitant du système glandulaire de l'appareil digestif est done suffisamment démontré et les effets pathologiques qui résultent d'une alimentation avitaminée peuvent bien être attribués non pas tant à l'absence des vitamines dans le sang, mais simplement à l'arrêt de la digestion et par là à l'inanition ou encore au passage dans le sang de produits incomplètement digérés.

Chaque aliment doit apporter avec lui sa vitamine pour être convenablement digéré et préparé ainsi à son assimilation, et si la spécificité fonctionnelle des différentes vitamines n'est pas pratiquement exclusive, cela tient probablement bien plus à ce fait, que ce sont les sucs digestifs qui ne sont pas spécifiques: que le suc gastrique, par exemple, digérera tout aussi bien l'albumine de l'œuf, d'une viande ou d'un légume, à la condition d'être excrété en quantité suffisante sous l'action d'une vitamine quelconque.

L'intervention des vitamines va-t-elle encore plus loin? Contribuent-elles, et dans quelle mesure, à la 
synthèse des tissus après avoir aidé à préparer les éléments qui doivent les nourrir? Agissent-elles directement sur les cellules intéressées ou par l'intermédiaire des centres nerveux ou des glandes endocrines?

Autant de questions qu'il nous faut encore laisser sans réponse.

Une dernière question se pose: l'intervention des vitamines comme facteur de l'évolution progressive.

A en juger par leur rôle dans la digestion chez les vertébrés supérieurs, par leur présence dans les tissus des protozoaires et des végétaux, on peut admettre que ce sont les vitamines qui ont provoqué la formation des ferments d'abord, et des organes sécrétoires de ces ferments digestifs ensuite, chez les animaux.

$\mathrm{Au}$ début de la formation des premières espèces animales, l'intervention des vitamines était donc d'une importance capitale. Depuis, rien ne nous donne à penser qu'elles aient changé de nature, qu'elles ont pu être, à un moment quelconque, les agents de la formation d'une forme d'énergie nouvelle.

Mais tout n'est peut-être pas dit à ce sujet. Il viendra peut-être un moment, quand nous connaîtrons mieux leurs propriétés chimiques et physiologiques et surtout leurs spécificités différentes, l'électivité de leur action sur tel ou tel autre organe ou tissu, où nous saurons utiliser leur activité dans des buts précis et en faire des agents d'un perfectionnement plus sûr et plus rapide. 


\section{2. - Résumé et conclusions du Livre II}

Les faits que nous venons d'indiquer dans les chapitres qui précèdent, nous permettent donc de conclure que si, considérée comme un composé chimique, la matière vivante subit les lois générales qui déterminent les réactions de la matière minérale composée des mêmes éléments simples, considérée comme unité physiologique, elle obéit, dans ses réactions, à des lois qui ne sont pas exclusivement du domaine de la chimie pure, minérale ou moléculaire.

La matière vivante est composée d'une trentaine d'éléments que nous retrouvons exactement les mêmes, dans le monde inorganique ; tout élément chimique introduit dans une cellule vivante provoque donc et subira les mêmes réactions chimiques qu'en présence des mêmes éléments du monde inorganique. L'oxygène provoquera. des phénomènes d'oxydation, le chlore formera des chlorures, le soufre des sulfites ou des sulfates, etc., dans une cellule vivante comme dans un liquide contenant les éléments qui composent la cellule; mais les effets seront très différents parce que, dans l'eau, ces éléments sont isolés, indépendants et inertes les uns pour les autres, tandis que dans une cellule ils sont groupés en unités de fonctions différentes, et que ces unités plus simples sont réunies en une unité de fonction d'ensemble.

On peut se faire une idée de la différence des effets produits dans les deux cas, par le même réactif, en faisant agir l'oxydation de l'essence sur une machine compliquée, par exemple une automobile, d'une part complètement démontée, dont les pièces sépa- 
rées seraient mises en tas; d'autre part, la même automobile prête à partir avec son chauffeur au volant. Dans le premier cas, l'essence allumée, en s'oxydant elle-même, fera brûler tout ce qui est combustible; dans l'autre, la même réaction chimique subie par l'essence conduira l'automobile à une destination déterminée. Dans les deux cas, la même réaction provoquée par la même substance (oxygène) aura donc produit deux résultats absolument incomparables et cette différence est due uniquement aux différences du degré d'organisation, ou, en d'autres termes, à l'état d'évolution du même groupe d'éléments chimiques.

Les différents organes de l'automobile peuvent donc être comparés à des molécules chimiques plus ou moins complexes, et l'automobile en marche comparé à une micelle vivante, serait une unité physiologique, comme l'admettait déjà Herbert Spencer, et cette distinction peut et doit être admise sans être une concession au "vitalisme ", comme semblent le croire certains biologistes. Une cellule, un animal sont aussi des " unités " chimiques, mais il ne viendrait à l'idée de personne de les appeler "molécules " ni de leur attribuer les mêmes propriétés globales qu'à une molécule chimique.

Tout phénomène vital, le plus simple comme le plus complexe, une contraction des pseudopodes d'une amibe, comme la pensée la plus élevée d'un savant, ont donc pour origine des réactions chimiques simples et peuvent avoir pour causes immédiates les mêmes réactions provoquées par les mêmes réactifs sur des éléments identiques quant à leur nature chimique; les effets ne seront pas comparables, parce que les mêmes réactifs agiront sur les 
mêmes éléments groupés en micelles différentes réu nies dans des cellules destinées à des fonctions différentes.

Dans les cellules cérébrales de l'homme, les réactions d'oxydation et de réduction provoquent des mouvements volontaires et des pensées consciente: qui amèneront toutes sortes d'actes volontaires et réfléchis. Dans le plasma d'une amibe, ces mêmes réactions n'auront d'autres résultats que de maintenir dans un certain état d'équilibre les fonctions de nutrition et de multiplication; dans une molécule chimique inorganique, la réaction chimique proprement dite restera simplement telle quelle.

Chaque unité nouvelle formée par synthèse inorganique ou par une différenciation biologique et douée d'une forme d'énergie nouvelle, a droit à un nom nouveau, tant pour caractériser sa structure que sa fonction. Noous avons vu (chap. Iv-rx) que dans les micelles l'affinité chimique n'obéit pas aux mêmes règles que dans les molécules, et ce serait inutilement compliquer et embrouiller les choses et les idées, que de s'efforcer à appeler du même nom des unités douées de propriétés d'un ordre différent.

En partant des colloïdes, qui ont donné naissance aux premières micelles albuminoïdes et par là aux cellules vivantes, que nous appellerons les premières unités biologiques, nous pouvons admettre que le premier facteur important de l'évolution progressive de la matière vivante a été la formation des vitamines par les protozoaires et par les végétaux. Nous avons vu, en effet, autant qu'il est possible d'en juger par les expériences faites jusqu'à présent, que c'est exclusivement grâce aux vitamines que les animaux, qui sont incapables de les produire eux-mêmes, peu- 
vent assimiler les albumines végétales, se nourrir ainsi de substances déjà sélectionnées et d'une structure plus élevée, acquérir par là une activité et une plasticité plus grande, qui leur permettent de subir l'influence des énergies rayonnantes et enfin de différencier le tissu nerveux.

Le deuxième facteur déterminant a été la formation par différenciation du " tissu nerveux », d'abord des organes des sens périphériques, ensuite des fibres nerveuses, puis des ganglions ou centres nerveux et enfin, dans ces centres, des sphères sensorielles et des sphères d'association de plus en plus complexes et étendues. Et nous avons vu qu'à mesure que les organismes devenaient de plus en plus complexes, parce que plus finement différenciés, ils devenaient en même temps, de plus en plus sensibles à des substances et à des formes d'énergies de plus en plus nombreuses et variées.

Ainsi, tous les êtres vivants, animaux, protozoaires ou végétaux sont à peu près également sensibles aux cristalloïdes simples (acides, bases, sels) ; les cristalloïdes complexes (glucosides, alcaloïdes) sont déjà d'une action plus élective, ils agissent moins ou à beaucoup plus forte dose sur les végétaux et les animaux inférieurs que sur les animaux supérieurs.

Les colloïdes, en tant qu'antigènes, ne provoquent probablement aucune réaction appréciable chez les végétaux saprophytes, peut-être agissent-ils d'une certaine façon sur certains êtres unicellulaires, sur les végétaux parasites, ainsi que sur les animaux inférieurs. La fonction de la formation des anticorps n'apparaît, semble-t-il, comme facteur de modifications importantes, que chez les vertébrés supérieurs: les oiseaux et les mammifères.

\section{http://rcin.org.pl}


Les énergies rayonnantes ne deviennent des facteurs dominants de l'évolution que chez les animaux pourvus d'un système nerveux différencié et alors, leur action, celle de la lumière et du son en particulier, sont d'autant plus manifestes que l'organe périphérique du sens et le centre sensoriel correspondant sont plus développés.

Les émotions ne commencent à être ressenties et à réagir que sur les animaux pourvus d'un cerveau et enfin la pensée proprement dite, la volonté consciente de ses buts n'apparaît et ne commence à réagir d'une façon appréciable sur l'évolution qu'avec la formation des sphères d'association de l'homme. L'apparition de la pensée délimite l'époque la plus importante dans le processus de l'évolution progressive, parce que cette forme d'énergie réagit non seulement sur l'évolution de l'unité " matière énergie " homme, sur son milieu intérieur, mais aussi, d'une façon indirecte, sur tout son milieu extérieur, sur toutes les unités "matière-énergie" qui ont précédé l'homme.

Bien entendu, ainsi que nous l'avons dit plus haut, toutes ces actions et réactions ont pour point de départ des réactions physico-chimiques et aboutissent à d'autres réactions de la même nature; il serait donc du plus haut intérêt d'en connaître le mécanisme intime.

Nous savons ce qui entre dans un organisme et sous quelle forme les substances qui y entrent sont assimilées par les cellules; nous pouvons analyser ou apprécier les produits qui en sortent, mais nous ignorons encore complètement quelles transformations subit chaque composé, quelles combinaisons chaque élément a formées pendant ce passage, parce que nous ne connaissons pas la constitution intime de 
l'unité physiologique de tout être vivant, de la gemmule de Darwin, plastidule d'Elsberg, pangènes de de Vries, plasome de Wiesner, idioblaste de Hertwig, biophore ou déterminant de Weismann, etc., en un mot de la micelle albuminoïde vivante qui ne peut, par définition, avoir exactement ni la même composition chimique ni la même structure que la micelle albuminoïde morte.

Nous avons vu que Clarke a fait pousser des ailes aux pucerons aptères (Aphis-rosæ), en arrosant les rosiers qui les nourrissaient avec un peu de sulfate de magnésium, que Stockard a obtenu des larves de. poissons (Fundulus hétéroclitus) munies d'un seul œil au milieu de la tête, en ajoutant un peu de chlorure de magnésium à l'eau de mer normale, mais nous ne savons ni pourquoi ni par quel mécanisme les sels de magnésium produisent des effets aussi différents et extraordinaires.

Savoir ce qui se passe, suivre les réactions qui aboutissent à la formation des ailes chez des pucerons qui normalement n'en ont pas, est d'autant plus difficile que le même effet peut être produit par d'autres substances telles que les sels de zinc, d'antimoine, de plomb ou le sucre qui, au point de vue chimique, présentent des différences très grandes. Et la seule conclusion que l'on puisse tirer actuellement de telles expériences, c'est que l'effet apparent résulte très probablement d'une série de réactions indirectes, d'une hypo ou hypersécrétion d'une glande qui, chez les animaux qui en sont pourvus, détermine la formation et le développement de tel ou tel autre organe ou tissu, ou bien une combinaison de la substance étrangère avec la hormone ou l'harmozone, déjà versée dans le sang ou les humeurs. 
L'effet produit sera passager ou durable; la modification, le nouveau caractère acquis sera individuel ou deviendra héréditaire, selon que la réaction provoquée sera plus ou moins durable et qu'elle aura exercé une modification correspondante dans les cellules reproductrices.

Nous avons vu que, de toutes les substances étrangères, ce sont les colloïdes et surtout les colloïdes organiques, les albumines qui, par la formation des anticorps, provoquent dans les organismes les modifications, les équilibres nouveaux, les plus durables, et alors deux éventualités sont possibles : ou bien le nouvel équilibre favorise le développement de l'individu et, s'il est transmissible, crée une race plus résistante, plus apte à se perpétuer, ou bien c'est le contraire, et alors la race peut disparaitre.

En tout cas, pour que l'individu ou sa race devienne plus résistante, il faut qu'il y ait entre les fonctions des différents organes de l'organisme un équilibre aussi parfait que possible. L'hypertrophie d'un organe peut être aussi néfaste que sa dégénérescence.

En un mot, on constate qu'un organisme ne pourrait fonctionner d'une façon absolument normale et rester toujours identique à lui-même que s'il se trouvait dans un milieu absolument invariable. Or, nous savons que cette condition n'est jamais réalisée dans la nature. Le milieu extérieur se modifie à chaque instant; mais nous avons vu aussi que la matière vivante ne subit pas d'une façon passive les influences du milieu extérieur. Elle oppose un milieu intérieur de plus en plus stable aux changements du milieu extérieur et s'en isole de mieux en mieux à mesure que ses unités biologiques - les individus -

\section{http://rcin.org.pl}


deviennent de plus en plus complexes, doués d'une énergie propre de plus en plus puissante.

Comparable en cela à un tourbillon doué d'une certaine force d'inertie qui lui permet de résister, dans une certaine mesure, aux changements du milieu dans lequel il évolue, l'unité vivante ne subit l'influence de ces changements qu'en fonction de cette force d'inertie, et l'effet produit par l'intervention d'une énergie étrangère sera toujours une résultante d'une série de réactions, dont le mécanisme nous est encore très imparfaitement connu.

Tout ce que nous pouvons en dire aujour'dhui, c'est que, chez les animaux supérieurs, toute réaction évolutive ascendante ou descendante, s'effectue soit sous l'action du système nerveux, soit (et c'est le cas le plus fréquent) par l'intermédiaire des glandes à sécrétion interne qui règlent les conditions de nutrition et par là de développement de tous les autres tissus et organes.

Chez un animal, un changement provoqué par un facteur du milieu extérieur ne peut devenir durable et transmissible qu'à la condition de provoquer dans les fonctions des glandes endocrines un équilibre nouveau durable. 


\section{LIVRE III}

\section{L'ÉVOLUTION DE L'INDIVIDU ET DE L'ESPĖCE}

\section{3. - Les phases de l'évolution}

Dans le précédent chapitre nous avons envisagé la nutrition dans son processus le plus général. Nous avons cherché à représenter la constitution et les propriétés fondamentales de la matière vivante, c'està-dire communes à toutes les cellules, et nous ne pouvions le faire qu'en les étudiant d'après une cellule idéale, nécessairement schématique.

Pour comprendre les lois générales de l'évolution de la matière vivante telles qu'elles ressortent de l'étude des propriétés des êtres vivants, qui ont peuplé et peuplent actuellement la terre, il nous faut examiner en détail le mécanisme et les réactions de la nutrition, d'une part, dans les différentes phases de l'évolution d'un organisme, d'autre part, d'après quelques types de différentes classes de protistes, de plantes et d'animaux d'une organisation de plus en plus élevée.

Dans l'évolution d'un être vivant on peut généralement distinguer trois phases successives:

$1^{\circ}$ Une période d'accroissement à partir du germe 
ou de I'œuf jusqu'à un certain maximum, que les êtres d'une espèce ne dépassent pas;

$2^{\circ}$ L'état adulte, pendant lequel l'organisme ne s'ćcroît plus, mais se maintient à un certain maximum et optimum de structure et de fonctions;

$3^{\circ}$ Une phase de sénescence, pendant laquelle l'sctivité vitale décroît progressivement et finit par s'errêter.

Ces trois périodes sont à peu près de la même durée pour les êtres appartenant à la même espèce; elles varient beaucoup d'une espèce à l'autre et leurs durées respectives ne sont pas les mêmes pour les différentes espèces.

Ainsi, d'après les données recueillies par Wesmann, une baleine vivrait quelques siècles et il serait difficile d'assigner une limite à sa croissance.

Un éléphant, un brochet, une carpe peuvent vive deux siècles. Chez l'éléphant, la durée de la vie enbryonnaire est de deux ans, celle de l'enfance et de l'adolescence de 24 ans. Le brochet et la carpe qui vivent aussi longtemps, arrivent à maturité sexuelle en un an.

Le cheval, le crapaud et le chat peuvent vivee 40 ans.

Le cochon et l'écrevisse, 20 ans.

Le merle, 18 ans.

La souris, 6 ans.

Parmi les insectes, il y a des espèces qui ne vivent que quelques heures, d'autres quelques années; leur vie à l'état adulte dure généralement beaucoup moins longtemps que leur vie larvaire, mais il y a des exceptions. La punaise se développe en quelques semaines et peut vivre plusieurs années à l'état d'imago.

Les plantes et, en particulier les arbres, peuvent 
vivre beaucoup plus longtemps; on admet que certains Adansonias des îles du. Cap Vert existent depuis plus de 60 siècles, mais ainsi que nous le verrons plus loin, un arbre, tout en représentant une unité d'évolution, ne peut pas être considéré comme un simple individu de la même catégorie qu'une mouche, un brochet ou un homme.

Pourquoi une souris devient-elle adulte en cinq ou six semaines, un cheval en trois ou quatre ans, un homme en 18 à 25 ans? Pourquoi les souris ne peuvent-elles vivre pendant plus de 6 ans, les chevaux 40 ans et les hommes 60 à 120 ans ?

C'est là un de ces phénomènes biologiques dont les lois ont été le moins établies jusqu'à présent, et pourtant, une étude approfondie des conditions de nutrition des individus appartenant à différentes espèces animales ou végétales, présentant des cycles d'évolution différents, serait peut-être le moyen le plus commode et le plus facile, en nous fournissant des termes de comparaison précis, de nous apprendre, pourquoi les tissus d'un organisme cessent de s'accroître à un moment donné et finissent par subir, à un autre moment, une dégénérescence sénile; pourquoi, en un mot, tout individu pluricellulaire qui naît d'un œuf fécondé, doit avoir un cycle d'évolution fermé d'une certaine durée.

Nous commençons à entrevoir chez les animaux supérieurs, que cette durée est sous la dépendance du système des glandes à sécrétions internes, que c'est l'abondance et probablement aussi la nature chimique de ces sécrétions qui, peu différentes chez

Daxysz. - La genèse de l'énergie psychique. 
les différentes espèces, mais variables avec l'âge de l'individu, détermine la durée des trois périodes de l'évolution et par là aussi, du cycle de la vie.

Mais, pour le moment, nous ne possédons pas encore de données assez précises pour aborder l'étude systématique de ce problème, nous ne pouvons que le poser et indiquer, que c'est dans la recherche des propriétés des sécrétions internes, des réactions, que les glandes endocrines éprouvent sous l'action de divers stimulants, que l'on trouvera à ce sujet les indications les plus fécondes.

L'étude des flores et des faunes qui ont vécu à la surface de la terre depuis les temps les plus reculés jusqu'à nos jours, nous a montré que les espèces ont subi, elles aussi, une évolution. Beaucoup d'espèces de plantes et d'animaux dont on retrouve les traces sous formes de squelettes, de débris d'empreintes ou de moules, dans des terrains de l'époque primaire, secondaire ou tertiaire, n'existent plus aujourd'hui, et il en est apparu d'autres, qui n'existaient pas auparavant.

On peut admettre qu'un certain nombre des espèces, qui n'existent plus, ont complètement disparu, que d'autres ont subi des transformations dont nous étudierons le mécanisme plus loin. Pour le moment, il s'agit de se rendre compte par l'analyse des faits connus, si l'espèce doit subir la même évolution cyclique que l'individu, si elle doit passer nécessairement par les phases de croissance, de stabilisation, de vieillesse et disparaître, ou bien si son existence doit être considérée comme théoriquement infinie. 
Nous examinerons ces détails avec plus de fruit à la fin de ce chapitre, après l'étude de quelques types d'évolution des plantes et des animaux.

\section{4. - Evolution d'une plante annuelle et d'une plante vivace}

Un grain de blé qui germe, pousse des racines qui s'enfoncent dans le sol et une tigelle qui s'élève dans l'air. Les racines, la tige et les feuilles sont formées de cellules accolées les unes aux autres et chaque cellule est composée d'une paroi cellulosique et d'un plasma.

$\mathrm{Au}$ point de vue chimique, il y a cette différence entre la paroi cellulaire et le plasma, que les parois sont formées d'une substances bien stảble, d'un hydrocarbone appelé la cellulose, insoluble dans l'eau et dans la plupart d'autres liquides, tandis que le plasma est une substance albuminoïde plus ou moins liquide. $\mathrm{Au}$ point de vue physiologique, la cellulose est l'élément de structure, le plasma l'élément de travail de la plante, de ses réactions de nutrition et de multiplication.

A mesure que la plante s'accroît par la multiplication de ses cellules, les parois de ces cellules, tendres et fines au début, deviennent de plus en plus épaisses par l'accumulation continue des éléments de structure stables et insolubles.

La formation de l'épi et des fleurs, ainsi que la maturation des cellules sexuelles, coïncide avec l'entrée d'une plante annuelle dans l'âge adulte, qui dure jusqu'au moment de la maturité des graines.

Alors commence la période de sénescence. Les parois cellulaires deviennent de plus en plus épaisses, 
les substances ligneuses de construction finissent par remplir presque complètement l'intérieur des' cellules; le passage des sucs nutritifs du sol jusqu'aux feuilles et l'épi, de même que les échanges nutritifs entre les celules deviennent de plus en plus difficiles. Toute la plante se dessèche et meurt.

Dans le cycle de l'évolution d'une plante annuelle, l'évolution cyclique de l'individu est donc déterminée par l'antagonisme entre les éléments de structure insolubles et les éléments de travail proprement dits. La fermeture du cycle ou la mort de la plante est la conséquence de l'accumulation des composés stables, qui ne peuvent être éliminés parce qu'ils sont insolubles.

Les finalistes diraient à ce propos, que la plante construit une charpente de plus en plus solide, pour que ses racines puissent vaincre la résistance du sol et s'y enfoncer à la recherche des sucs nutritifs, pour que la tige puisse supporter le poids de l'épi chargé de graines. Tout se passe, en effet, comme si la plante de blé s'obligeait à subir une évolution déterminée pour transformer en paille sèche et en grains, bons pour la nourriture, les substances minérales contenues dans le sol et que nous ne saurions utiliser sous cette forme; mais n'est-il pas évident que la plante ne raisonne pas ainsi et qu'une intelligence supérieure qui dirigerait le monde n'aurait pas besoin de toute cette mise en scène, bien compliquée, pour transformer les éléments inorganiques en acides aminés et en vitamines, qui nous sont indispensables pour construire nos tissus?

Il est bien simple d'admettre, jusqu'à nouvel ordre, que l'évolution cyclique d'une plante annuelle est déterminée par les propriétés chimiques des éléments 
dont elle est composée, que les réactions toutes naturelles et obligatoires entre ces éléments ont pour effet de former certains composés insolubles, que précisément parce qu'ils sont insolubles, ces composés ne peuvent pas être éliminés et s'accumulent à la surface du plasma où les courânts sont moins rapides, qu'en un mot, il suffirait de connaître exactement toutes les combinaisons que les éléments, qui entrent dans la composition du blé doivent obligatoirement former entre eux, dans des conditions déterminées, pour expliquer comment et même pourquoi un grain de blé doit germer, se développer en plante et mourir dans un temps déterminé.

Et disons-le en passant, il n'est pas du tout indifférent d'envisager ce problème de cette façon, parce que ce n'est qu'en raisonnant ainsi, que nous aurons le désir de chercher et que nous pourrons peut-être arriver un jour à remplacer le blé par un produit de synthèse tout aussi nutritif et à échapper ainsi aux nécessités et aux aléas que la nature nous impose encore aujourd'hui.

L'évolution d'une plante vivace, d'un arbre, considéré comme un individu, est, en réalité, toute différente. On voit les arbres grandir, vieillir et mourir, et pourtant, en théorie, un arbre pourrait vivre éternellement.

Pour expliquer cette contradiction apparente, il faut se rappeler qu'un arbre s'accroît en épaisseur et en hauteur, en formant chaque année une nouvelle couche de cellules entre le bois et l'écorce. Cette couche de cellules appelée génératrice, est la seule 
partie vivante de l'arbre. Les cellules qui forment les couches plus profondes du bois et plus périphériques de l'écorce, s'épaissisent et meurent comme dans la tige du blé.

Toutes les cellules formées dans le courant d'une année proviennent d'une couche unique de cellules génératrices, elles sont donc toutes de la même génération. L'ensemble de la couche d'une année peut donc être considérée comme une plante annuelle distincte, de sorte qu'au point de vue de sa structure, un arbre de 50 ans, par exemple, peut être considéré comme formé de 50 plantes annuelles emboitées les unes dans les autres. La dernière formée est la plus éloignée du centre et la seule réellement vivante.

Au point de vue de son évolution, un arbre de 50 ans serait done bien plutôt une colonie ou une " espèce " formée de 50 générations successives de la même plante, qu'un individu.

Comme individu, c'est-à-dire comme couche de cellules d'une génération annuelle, un arbre subit les lois de l'évolution de tout autre individu pluricellulaire, il s'accroît jusquu'à un certain maximum, passe par une phase d'état adulte, vieillit et mẹrt. Considéré comme une colonie formée d'un certain nombre de générations successives, il pourrait vivre infiniment si un cataclysme ou une maladie ne venait le détruire.

S'il pouvait vivre assez longtemps, chaque arbre finirait par former une sorte d'enceinte circulaire couronnée de branches, enfermant un espace vide. Chaque année cette enceinte s'éloignerait de quelques millimètres du centre, l'espace vide deviendrait plus grand, et si des obstacles, impossibles à vaincre, venaient à empêcher, dans certains endroits, son 
accroissement périphérique régulier, l'enceinte serait rompue, les tronçons séparés pourraient continuer à vivre chacun pour son compte et un arbre deviendrait une colonie d'arbres, dans le sens propre de ce mot.

Il y a, dans la forêt de Fontainebleau, un chêne qui vit, dit-on, depuis au moins quatorze siècles, et il y a des eucalyptus en Tasmanie, des adansonias qui vivent, dit-on, depuis 6.000 ans; des baobabs sous les tropiques, dont les trones ont plus de 20 mètres de circonférence, qui sont certainement bien plus âgés que notre chêne, et il arrivera certainement à ces géants des accidents qui les feront mourir.

Le chêné de Fontainebleau a déjà perdu une de ses plus grosses branches, cassée par un orage. La plaie ainsi formée, permet à l'oxygène de l'air de décomposer le bois de la branehe d'abord, du tronc ensuite. Le bois décomposé sera attaqué par les mierobes, qui s'adapteront progressivement à se nourrir aux dépens du bois vivant. Le tronc deviendra creux et trop faible pour soutenir le poids des branches; le moindre orage le fera alors se déchirer et tomber en morceaux et telle sera, fatalement, la fin de toute "colonie-arbre" qui évolue librement dans la forêt.

Un arbre pourrait donc vivre éternellement, même s'il ne pouvait s'aceroître et se multiplier que par des bipartitions successives de ses cellules génératrices, parce qu'il forme constamment des couches nouvelles de cellules jeunes, et parce que ses éléments ou unités de multiplication, toujours renouvelés, ne sont pas gênés dans leurs évolutions successives par les éléments de structure qui les soutiennent au contraire et les disposent dans un ordre 
déterminé. Et si, en réalité, il finit toujours par mourir et disparaître, c'est uniquement parce qu'il ne peut pas se soustraire aux conditions de son milieu extérieur, qui deviennent avec le temps, toujours défavorables à son existence.

Pour bien faire ressortir l'importance biologique du fait qu'une plante annuelle est un individu à évolution eyclique, tandis que la vie d'un arbre peut être infinie, il est nécessaire de l'envisager encore à un autre point de vue.

Dans l'un comme dans l'autre, ce sont les cellules génératrices qui constituent la partie vivante, active du végétal, c'est par la multiplication, par simples divisions de ces cellules, que l'un et l'autre s'accroissent et se développent; on doit done se demander pourquoi cette multiplication s'arrête dans la plante de blé à un moment donné, et pourquoi elle continue d'année en année chez l'arbre?

Nous avons cherché à expliquer la mort de la plante annuelle par l'accumulation des éléments de structure dans les cellules, mais alors il faut admettre que les choses ne se passent pas de la même façon dans l'arbre, et chercher pourquoi chez les plantes vivaces les éléments de structure ne s'accumulent pas dans'les cellules de la dernière génération, de façon à arrêter leur multiplication ultérieure, comme cela se produit dans les plantes annuelles.

Pour trouver une explication satisfaisante à cette question, il nous faut la transporter sur un autre terrain, la simplifier encore en étudiant la multiplication des êtres unicellulaires. 


\section{5. - Multiplication des êtres « unicellulaires »}

Toutes les cellules qui composent un chêne plusieurs fois séculaire, comme celles qui composent une plante de blé âgée de quelques mois, proviennent d'une seule cellule génératrice. Dans le premier cas, cette cellule donne naissance, par simple division, à une série d'autres cellules génératrices qui peuvent se multiplier à l'infini; un arbre peut s'accroître et se multiplier indéfiniment sans intervention d'un ovule fécondé, dans l'autre cas, la cellule génératrice ne peut donner naissance qu'à un nombre limité de cellules filles qui finissent par mourir, de sorte que l'individu ne peut se reproduire que par des, graines, c'est-à-dire par des ovules fécondés, qui subiront un nouveau cycle d'évolution fermée.

Chez les êtres unicellulaires, la question de multiplication par simple division et l'intervention nécessaire d'une conjugaison, c'est-à-dire de la formation d'un œuf fécondé, a fait l'objet de nombreuses discussions et a suscité quelques recherches expérimentales intéressantes.

Weismann l'a posé le premier, d'une façon précise, sans chercher à confirmer ses idées par des expériences. Partant de ce fait que les infusoires se multiplient le plus souvent par simples divisions successives, sans qu'il y ait jamais, par suite de cette multiplication, des individus vieillis et des cadavres, Weismann a admis que les êtres unicellulaires peuvent se multiplier ainsi à l'infini.

Maupas a cherché à vérifier cette simple vue d'esprit en cultivant une vingtaine d'espèces d'infusoires différents, de façon à empêcher les conjugai- 
sons, et il a constaté que, dans les conditions, dans lesquelles il s'était placé, ses infusoires pouvaient vivre et se multiplier pendant quelques semaines ou quelques mois, mais finissảient toujours par vieillir et par mourir.

Les expériences de Maupas (1888-89) semblaient tellement démonstratives, que sa théorie : "L'impossibilité de la multiplieation indéfinie par division, sans régénération de l'espèce par conjugaison et formation d'ovules ", avait été généralement admise en biologie.

Cette théorie a été ensuite confirmée par Calkins (1902) qui a introduit dans cette étude un facteur nouveau. En ajoutant au milieu de culture (infusion de foin) des substances considérées comme stimulantes, tels que certains sels ou des extraits de viande, il a obtenu 700 générations par simples divisions; mais, tandis que les descendants de la dernière génération par division ont tous succombé, ceux qui ont pu se conjuguer ont donné naissance à une nouvelle génération vigoureuse.

Ensuite vient une série de travaux dont les résultats mettent en doute l'exactitude de l'interprétation donnée aux expériences de Maupas et de Calkins.

Enriquez, Metalnikoff, Woodruff, ont réussi à cultiver des paramécies (P. Aurelia, P. Candatum) pendant une longue série d'années : Woodruff cultive ses infusoires depuis 1907 jusqu'à nos jours et a obtenu ainsi plus de 5.000 générations successives, sans conjugaison et sans jamais observer un signe quelconque de vieillissement, et ces auteurs s'accordent pour conclure, que si leurs prédécesseurs ont vu périr leurs cultures, le vieillissement et la mort de leurs infusoires n'étaient pas dus à l'épui-

\section{http://rcin.org.pl}


sement progressif de la “ force vitale » qui doit être renouvelée par la conjugaison, mais uniquement à des poisons qui se forment dans leur milieu de culture : leurs propres sécrétions et les microbes plus ou moins nocifs.

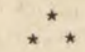

Un infusoire et, en particulier, une paramécie, est un organisme qui, bien qu'unicellulaire, est déjà assez complexe. Une paramécie est déjà pourvue d'une bouche, d'un rudiment de canal digestif et d'une vésicule pulsatile qui se contracte et se relâche périodiquement, et provoque ainsi un certain mouvement régulier des substances qui s'accumulent dans son corps. Il est assez difficile de préserver une culture de ces infusoires de contamination par des microbes nuisibles et enfin sa multiplication est relativement lente. Une paramécie ne donne guère plus de 300 générations par an.

On peut encore simplifier l'étude de ce problème en s'adressant à des êtres moins compliqués et notamment à des microbes.

\section{6. - Multiplication des microbes}

Expérience. - Si on eultive en culture pure un microbe assez commun, une race du "bacillus paratyphosus " (microbe qui ne forme jamais de spores, autant qu'on a pu le constater jusqu'à présent) dans un bouillon préparé avec une décoction de viande additionnée d'un peu de peptone, en le réensemencant autant que possible tous les jours dans un peu de ce bouillon frais, préalablement stérilisé, on constate le fait suivant. 
En ensemençant 100 microbes dans 10 centilitres de bouillon et en plaçant le tube de culture dans une étuve à température constante de $37^{\circ}$, on obtient, en 24 heures, 25 à 30.000 microbes.

En admettant que tous les microbes se soient multipliés également par bipartition, on constate que chaque microbe aura donné en 24 heures neuf générations successives, ce qui fera, en chiffres ronds, 3.000 générations par an.

On a cultivé ce microbe sans interruption pendant cinq années successives, on a donc obtenu 15.000 générations et jamais on n'a observé un signe quelconque de sénescence; bien au contraire, les microbes réensemencés chaque jour dans un bouillon frais, mais de composition identique, s'adaptaient de mieux en mieux à ce milieu et se multipliaient de plus en plus rapidement, de sorte que, au bout d'un certain temps, on obtenait neuf générations en 20 heures, puis en 16 et enfin en 12 heures.

En renouvelant le bouillon toutes les 20, 16 ou 12 heures, on obtiendrait donc, au bout de cinq ans, un nombre de générations beaucoup plus considérable.

Or, on peut bien admettre que si un microbe peut être cultivé pendant cinq années consécutives et donner naissance au moins à 15.000 générations sans rien perdre de sa vitalité et de ses propriétés appréciables (il n'a même pas varié dans sa virulence pour les souris), il pourrait vivre et se multiplier dans les mêmes conditions pendant une autre période de cinq ou de cinquante années et même de cinquante siècles. Il est certain, que si on le maintient toujours dans les mêmes conditions de milieu (bouillon, température, lumière, aération) et si on renouvelait le 
bouillon nutritif assez souvent, il pourrait vivre et se multiplier à l'infini et rester toujours identique à lui-même.

Toute autre será l'évolution du même microbe, si on le laisse se développer dans le même milieu sans le renouveler. Il donnera toujours neuf générations pendant les premières 24 heures, mais seulement une génération pendant la deuxième journée : en tout, pour 100 microbes ensemencés, 45 à 50.000 individus en 48 heures, et on constatera que, au bout de la troisième journée, le nombre total de microbes sera inférieur à 45.000. Dans, le cours de la troisième journée, le nombre des décès sera donc supérieur à celui des naissances. La multiplication ne s'est pas arrêtée. On trouve toujours des microbes bien vivants et vigoureux, en train de se diviser, mais puisque le nombre total de corps microbiens a diminué, il faut bien en conclure que ceux qui manquent ont été complètement détruits par cytolyse.

En maintenant la même culture pendant plus longtemps, dans le même milieu non renouvelé, on observe plusieurs faits qu'il est intéressant de faire ressortir ici.

Les sucres (glucose et probablement aussi un peu de lactose), provenant de la viande avec laquélle on a fait le bouillon, sont transformés en acides. Le milieu devient donc d'abord plus acide. La peptone et autres protéoses sont transformées en acidesaminés, en amines et en ammoniaque qui neutralisent d'abord les acides et ensuite rendent le milieu de plus en plus alcalin.

Les microbes qui vivent près de la surface et ont à leur disposition plus d'oxygène que ceux qui vivent 
au fond, se multiplient plus rapidement, finissent par s'y accumuler et par former un voile qui empêche l'arrivée de l'oxygène de l'air aux couches plus profondes.

Un grand nombre de microbes sont détruits par la cytolyse et les substances organiques et inorganiques mises ainsi en liberté et versées dans le milieu nutritif servent à la construetion du plasma des générations nouvelles.

Il se passe encore là beaucoup d'autres réactions de décomposition et de synthèse, dont nous sommes loin de connaître tous les détails, mais dont nous pouvons apprécier les résultats.

Les microbes modifient peu à peu la composition du milieu qui, à son tour, modifie la constitution physico-chimique, ainsi que les propriétés des microbes. Les uns s'adaptent à un milieu plus acide, d'autres à un milieu plus alcalin. Ceux qui forment le voile à la surface du bouillon s'adaptent à la vie aérobie; ceux qui se multiplient dans les couches plus profondes du liquide, deviennent anaérobies. Un grand nombre sont détruits; d'autres passent à l'état de vie latente (sans former des spores) et peuvent rester ainsi sans changer, pendant des semaines et des mois.

$\mathrm{Au}$ bout de quelques semaines, on trouvera donc dans le bouillon ensemencé avec une seule race de microbes identiques entre eux (provenant d'une seule colonie) toute une série de races ou de variétés très différentes, tant au point de vue de leurs formes qu'à celui de leur constitution physico-chimique et de leurs propriétés biologiques.

En prolongeant l'expérience pendant quelques mois, on verra la multiplication des microbes s'ar- 
rêter définitivement. Les substances nutritives du bouillon seront complètement transformées et épuisées. Tous les microbes encore vivants passeront à l'état de vie latente et ne tarderont pas à mourir, - principalement à cause de l'alcalinité du milieu.

Si on cultivait les mêmes microbes dans des bouillons contenant, d'une part, des quantités progressivement croissantes de sucre, d'autre part, de peptone, en présence ou à l'abri de l'air, on obtiendrait quatre races ou variétés et, avec le temps, probablement, des espèces nettement différentes.

Je ne connais pas d'expérience qui aurait été faite spécialement dans ce but avec le B. paratyphosus, mais j'ai cultivé le microbe du charbon dans son milieu nutritif ordinaire (bouillon de viande peptonisé) en ajoutant à ce bouillon, d'une part, des quantités croissantes d'acide arsénique, d'autre part, du chlorydrate de quinine.

Au début de l'expérience, le microbe était tué par coagulation, dans une solution de 1 p. 500 d'acide arsénique, mais peu à peu on a pu l'habituer à vivre et à donner des cultures abondantes dans une solution contenant 1 p. 400 d'acide arsénique.

Dans ces cultures, il était impossible de retrouver les caractères distinctifs des bactéridies charbonneuses, les bâtonnets compacts, à bouts earrés bien nets, ont été transformés en cellules à bouts arrondis, remplis de vacuoles ressemblant à des cellules de levure et les bâtonnets droits sont devenus des filaments contournés en ressorts à boudin et entourés d'un gros manchon de matière mucillagineuse. Dans la culture sur gélose, la masse mucillagineuse se développe sur une épaisseur de deux à trois millimètres. Les miorobes ainsi transformés ressemblaient

\section{http://rcin.org.pl}


bien plus aux bactéroïdes des légumineuses ou à des nostocs qu'aux bactéridies charbonneuses.

Dans la solution de quinine, les microbes et les filaments devenaient, au contraire, de plus en plus fins, les cultures sur gélose plus sèches.

Les caractères nouveaux acquis par les bactéridies cultivées dans les solutions d'acide arsénique ou de quinine se conservaient ensuite pendant une longue série de générations, dans des milieux de culture ordinaires dépourvus d'arsenic ou de quinine.

Les̀ microbes transformés ont complètement perdu leur virulence, de sorte qu'en considérant l'ensemble des caractères morphologiques et biologiques acquis par ces deux cultures, il serait impossible de deviner leur origine commune. Tout bactériólogiste non prévenu serait obligé de eataloguer la bactéridie nor-. male et ses deux formes transformées, comme trois espèces différentes.

Ainsi, en donnant aux microbes une nourriture suffisante et assez souvent renouvelée, la vie de l'espèce peut être infinie et les individus auront toujours les mêmes formes et les mêmes propriétés. En les gardant toujours dans le même milieu non renouvelé, ils subiront des transformations variées et finiront par disparaître.

En les cultivant dans des milieux différents, on obtinedra des espèces nouvelles, que l'on peut "fixer" et faire vivre, sous cette nouvelle forme, d'une façon indéfinie.

\section{7. - L'individu et l'espèce}

Au point de vue de l'évolution de l'individu et de l'espèce, il est encore intéressant de faire remarquer 
ici, qu'en ce qui concerne les microbes et, en général, les êtres unicellulaires qui se multiplient par des bipartitions successives, il est impossible d'établir une distinction entre l'individu et l'espèce.

Quand on considère l'évolution, la vie d'une plante annuelle ou d'un animal, on n'éprouve aucune difficulté à distinguer et à définir l'individu et l'espèce. Dans ces cas, l'individu c'est l'organisme qui naît, s'accroît, vieillit et disparaît comme unité distincte; il a une certaine durée et un corps qui, après la mort, devient un cadavre.

L'espèce, c'est l'ensemble de ces individus présentant certains caractères communs, qui vivent à un moment donné, ont vécu auparavant et vivront après.

Or, pour tous les microbes et pour beaucoup d'autres organismes unicellulaires qui se multiplient par une série de bipartitions successives, il est impossible, comme l'a déjà fait remarquer Weismann, d'établir toutes ces distinctions. Le microbe $n^{\circ} 1$ se divise en deux microbes qui porteront le $n^{\circ} 2$, et ces derniers se diviseront chacun en deux et donneront quatre microbes $n^{\circ} 3$. Ensuite, il y aura huit microbes $n^{\circ}$ 4, 16 microbes $n^{\circ} 5$ et ainsi de suite...

Nous aurons ainsi, en apparence, cinq générations successives, mais en réalité, comme les deux individus du $n^{\circ} 2$ contiennent la substance du $n^{\circ} 1$, les quatre du $n^{\circ} 3$ contiennent la substance des unités du $n^{\circ} 2$, les huit du $n^{\circ} 4$ celle des quatre unités du $n^{\circ} 3$, les 16 unités de la cinquième génération représentent la somme des individus de toutes les générations précédentes. Ils sont tous du même âge; il n'y a ni vieillissement, ni mort pour aucun d'eux, de sorte que, tous les individus provenant de la multi-

Dixysz. - La genèse de l'éner pie psychique. 
plication d'un seul microbe, pris à un moment quelconque de leur évolution, ne représenteront, en réalité, qu'une seule génération, et même, à proprement parler, un seul individu divisé en une quantité queleonque d'unités identiques, libres.

On peut donc concevoir un microbe comme un seul étre à croissance continue et illimitée, dont les unités de nutrition biologiques, à dimensions limitées, deviennent libres et indépendantes les unes des autres, à mesure qu'elles se forment.

Si les conditions de milieu restaient toujours les mêmes, ces unités resteraient toujours, elles aussi, identiques à elles-mêmes, précisément parce qu'étant libres et indépendantes les unes des autres, elles peuvent vivre dans des conditions de nutrition identiques. Si, au lieu de devenir libres, les unités successivement formées restaient intimement unies les unes aux autres, de façon à former une masse compacte, les conditions de nutrition des unités des différentes couches deviendraient différentes, et il en résulterait une différenciation de formes et de propriétés qui conduirait à la formation d'un organisme complexe. Cet organisme aurait alors une évolution d'ensemble cyclique comme celle d'une plante annuelle ou d'un animal, ou même de la couche génératrice annuelle d'un arbre, parce que l'ensemble des cellules réunies en une unité distincte, formeront un milieu intérieur invariable, ou en tous cas peu variable, et se trouveront ainsi dans des conditions analogues à celles de nos microbes cultivés dans le même bouillon non renouvelé (p. 221). 


\section{8. - Formes de résistance. Conjugaison}

Ces quelques exemples de multiplication de croissance et d'évolution des microbes, des infusoires, des plantes annuelles, et enfin des plantes vivaces, nous permettent maintenant de jeter un coup d'œil d'ensemble sur quelques règles fondamentales de l'évolution de la matière vivante et de trouver des explications suffisamment claires sinon définitives, des phénomènes que nous avons signalés en passant, sans pouvoir en indiquer en même temps la véritable signification biologique.

Nous avons donc constaté que chez une plante vivace la cellule génératrice, dont l'origine peut être quelconque (racine, feuille, rameau ou tige), peut se multiplier à l'infini, mais on sait que l'arbre peut se multiplier aussi par la formation et la fécondation des cellules sexuelles spéciales; que chez les plantes annuelles l'évolution de cette cellule générátrice est cycliquue et que ces plantes ne peuvent se multiplier que par des cellules sexuelles spéciales; que les infusoires peuvent se multiplier par simples divisions, mais aussi par des ovules fécondés, mais dans ce cas, il n'y a pas de cellules sexuelles spéciales: les deux individus qui se conjuguent ne diffèrent, en apparence du moins, en rien de ceux qui ne se conjuguent pas; qu'enfin, chez les microbes, la multiplication par division est infinie et qu'il n'y a pas de conjugaison, mais des formes de résistance ou des spores.

Or, il suffit de bien se représenter la signification biologique d'une spore, ainsi que les conditions dans lesquelles elle se forme, pour comprendre la signification biologique d'une spore, ainsi que les condi- 
lesquelles elle se forme, pour comprendre l'origine et la signification de l'acte de conjugaison chez les infusoires, de la fécondation et de la formation de l'œuf chez les plantes pluricellulaires ainsi que chez les animaux.

Les microbes prennent des formes de résistance quand les conditions de nutrition leur deviennent insuffisantes ou défavorables. Et alors que se passe$t$-il? Le microbe, au lieu de se diviser en deux individus distincts, reste "un " et s'entoure d'une membrane plus résistante. Y a-t-il la fusion de deux individus qui devraient se séparer? C'est difficile à dire parce que nous ne connaissons encore rien de la structure intime du corps microbien, mais c'est très probable. Un microbe qui subit une division en deux individus distincts toutes les deux heures, se trouve pour ainsi dire toujours en état de division plus ou moins avancé. Mais est-il possible de trouver une différence fondamentale entre le fait de la réunion en une spore, de deux individus qui allaient se diviser, et celui de la réunion de deux individus distincts qui sont déjà devenus libres? Certainement non. Nous avons vu, en effet, que tous les individus microbes d'une même culture, vivant à un moment donné, sont du même âge et doivent être identiques entre eux.

On peut en dire tout autant d'une culture d'infusoires provenant d'un seul individu et élevés dans le même récipient.

Dans ces deux cas, ce sont les conditions du milieu extérieur qui ont déterminé à l'origine la formation des espèces qui se multiplient exclusivement par bipartition, par la formation des spores ou des œufs.

Chez les végétaux pluricellulaires différenciés, ces phénomènes sont certainement beaucoup plus com- 
plexes parce que, dans ce cas, il y a un milieu intérieur déjà bien différencié, qui résiste dans une certaine mesure et plus ou moins bien, chez les différentes espèces, aux influences du milieu extérieur, et qui détermine les conditions de l'évolution des cellules sexuelles spéciales.

C'est dans la différenciation plus ou moins grande et dans le développement plus ou moins considérable de certains tissus qui permettent à la plante de résister mieux ou moins bien aux agents du milieu extérieur (humidité, température, etc.) que l'on doit chercher les causes de l'évolution cyclique du blé et de l'évolution indéfinie d'un arbre.

Quant à la formation des cellules sexuelles différenciées et différentes pour les deux sexes, nous ne possédons pas encore les éléments nécessaires pour en établir les causes exactes, mais il semble certain qu'elle a aussĩ la même origine que la formation des spores chez les microbes. Une graine ne serait autre chose qu'une forme de résistance formée primitivement dans des conditions de nutrition défavorables, et qui peut rester inactive jusqu'au moment où ces conditions lui permettront de recommencer un nouveau cyele d'évolution.

Dans les expériences de Maupas et de Calkins il y a un fait qui n'a pas suffisamment attiré l'attention de leurs contradicteurs. Ces biologistes ont constaté, que si les infusoires issus de bipartitions successives finissaient toujours par vieillir et par disparaître, ce qui, suivant Metalnikoff, Woodruff, etc., était dû à des conditions de milieu défectueuses; les individus issus de la conjugaison de la dernière génération disparue pouvaient pourtant recommencer un nouveau cycle d'évolution par bipartition, et cela 
dans les mêmes conditions de milieu, dans lesquelles les premiers ne pouvaient plus vivre.

Il y a donc là quelque chose de plus qu'une rénovation. Il semble qu'il faut chercher dans le phénomène de conjugaison une sorte de processus d'immunité ou d'adaptation, qui fait présumer une modification préalable de l'équilibre nutritif et un changement de chimiotaxie qui a pour conséquence la réunion en une spore de deux microbes qui devraient se séparer, de deux paramécies en un œuf, dont les descendants sont immunisés contre un milieu, dans lequel ses ascendants ne pouvaient plus vivre.

Chez les plantes et les animaux pourvus d'un milieu intérieur différencié et qui forment des cellules sexuelles spéciales, cette formation serait l'effet d'une immunisation à deux degrés, contre le milieu extérieur d'abord et le milieu intérieur ensuite.

\section{9. - Accroissement continu}

Ainsi, on peut eoncevoir l'évolution des êtres unicellulaires et des végétaux comme un accroissement continu de la matière vivante par la multiplication de ses unités de nutrition: unités physiologiques ou micelles albuminoïdes qui, par leur réunion, puis par leur différenciation, forment d'abord des unités biologiques, des cellules isolées et libres, et ensuite, par la réunion et la différenciation de ces dernières, des organismes pluricellulaires pourvus de tissus et d'organes différents.

Les dimensions des cellules sont limitées et alors, si les conditions du milieu extérieur (température, quantité et qualité des substances nutritives) sont 
telles, que la cellule peut conserver un certain équilibre physico-chimique qui lui permet l'accroissement simultané et bien proportionné de ses éléments de travail et de structure, du plasma et de la membrane d'enveloppe, la cellule pourra se multiplier indéfiniment par une série ininterrompue de simples divisions successives; mais si les conditions de milieu se modifient de façon à favoriser le développement de la membrane et à empêcher le développement concordant du plasma, il y aura formation des formes de résistance, des spores, telle que nous le voyons chez les microbes, et il est très probable que c'est dans ce processus de la formation des spores que l'on doit chercher l'origine et le principe fondamental de la formation des oospores chez les infusoires, des cellules sexuelles spéciales et des œufs fécondés chez les organismes pluricellulaires.

Théoriquement, un arbre pourrait vivre et se multiplier indéfiniment par la seule multiplication de ses cellules génératrices et l'on pourrait certainement réaliser les mêmes conditions de multiplication indéfinie pour toutes les plantes annuelles. On peut donc admettre que dans tout le règne des protistes et des végétaux, le processus de la fécondation et la formation des formes de résistance n'est pas une propriété fondamentale de la matière vivante procédant de sa composition élémentaire, de sa structure et de ses règles d'évolution, mais des conditions biologiques déterminées par les variations du milieu extérieur.

Mais, ainsi que nous le verrons dans le chapitre qui suit, le processus de multiplication par des œufs fécondés dans les organismes pluricellulaires, devient peu à peu le facteur le plus important de la continuité de l'évolution progressive. 


\section{0. - Evolution de l'individu animal}

Les animaux, de même que les végétaux, tirent leur origine des êtres unicellulaires; chaque individu animal provient d'une seule cellule; les règles générales de l'évolution de la matière vivante sont donc les mêmes pour les uns et les autres.

Nous avons vu, dans les chapitres précédents, que le facteur décisif de l'évolution progressive des animaux était la différenciation du tissu nerveux et que cette différenciation a été précédée et déterminée par la vie, pour ainsi dire, parasitaire des protistes qui, par une suite de formations dont nous ne connaissons pas encore tous les chaînons, ont donné naissance à des animaux.

L'ovule fécondé d'un végétal donne naissance, en se multipliant par une série de bipartitions successives, à un certain nombre de cellules génératrices qui, en continuant à se multiplier de la même façon, produisent, d'une part, la tige avec ses rameaux, ses feuilles, fleurs et fruits, d'autre part, les racines avec leurs radicelles. Un être unicellulaire quelconque pourrait évoluer en une plante de la même façon par une juxtaposition de toutes les cellules filles dans un ordre déterminé, suivie d'une différenciation rendue obligatoire, précisément, par cette juxtaposition. Pour qu'un être unicellulaire ait pu devenir animal, le processus devait êtré plus complexe.

A en juger par ce que nous savons des premiers stades du développement de l'œuf fécondé d'un animal, ce sont les protozoaires qui sont devenus des "gastrulas " ou, en d'autres termes, qui en se multipliant et en restant accolés les uns aux autres, ont 
formé un groupe de cellules, dont l'ensemble était pourvu d'une ouverture et d'une cavité intérieure, qui seules ont pu devenir les prototypes des animaux.

L'ouverture c'était la bouche primitive, la cavité, le rudiment de l'appareil qui pouvait garder pendant un certain temps et digérer les particules albuminoïdes ingérées, pour les rendre assimilables.

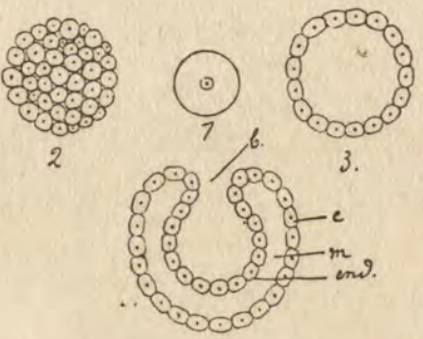

Fig. 26.

Premiers stades du développement de l'oosperme chez les animaux

L'ovule fécondé (1) se divise successivement en 2, 4,8, etc. cellules qui forment tout d'abord une sphère pleine : la morula (2). Cette dernière se transforme en une sphère creuse : la blastula (3), dont la phériphérie est formée d'une seule couche de cellules. La blastula se transforme en gastrula (4), par invagination d'une partie de sa paroi de façon à former un sac, muni d'une ouverture (b) qui deviendra la bouche. La paroi extérieure du sae deviendra l'ecloderme (e), la paroi intérieure, l'endoderme (end). Dans l'espace intermédiaire se formera le mésoderme (m) de l'embryon.

L'œuf d'un végétal n'arrive pas au stade de gastrula et c'est là la cause de son infériorité originelle dans l'échelle des êtres vivants, parce que c'est précisément ce stade d'appareil digestif primitif invaginé, qui a permis à l'organisme de se nourrir de matériaux complexes tout faits, de substances albuminoïdes accompagnées de leurs vitamines et, par là, de faire prendre à l'évolution de la matière vivante 
une direction ncuvelle qui l'a conduite à la forme animale.

G'est donc la formation d'un organisme possédant la structure et les fonctions d'une gastrula, qui peut être considérée comme le pas, le facteur décisif, par lequel l'évolution progressive de la matière vivante a été dirigée dans une voie nouvelle, dans celle qui l'a conduite chez l'animal, à la différenciation du tissu nerveux.

Nous n'avons pas à nous occuper ici de la filiation des animaux depuis le prototype unicellulaire jusqu'à l'homme, que nous avons d'ailleurs indiqué succinctement en parlant de l'évolution du tissu nerveux. Nous n'avons d'autre intention ici, que celle de trouver une définition aussi exacte que possible de l'individu et de l'espèce par rapport au rôle de l'un et de l'autre dans l'évolution progressive.

Nous avons vu que chez les protozoaires et les végétaux il n'y a pas d'individus, à proprement parler. S'il n'y avait pas d'animaux à la surface de la terre, on ne pourrait donner le nom d'individu qu'à l'ensemble de la matière vivante formée d'une infinité d'unités de réactions libres, d'une structure variable se multipliant infiniment par divisions successives.

L'individu vivant, proprement dit, n'apparaît que sous forme "animal ", et encore il est à noter que chez les animaux l'individualisation est fonction du développement du système nerveux. Au plus bas de l'échelle, chez les porifères, les cœlentérés et même chez quelques vers inférieurs, une partie quelconque 
séparée du corps de l'animal peut reproduire le corps tout entier. Ces animaux pourraient donc se reproduire par simple division de cellules somatiques, sans intervention des cellules sexuelles. Ce serait encore l'accroissement de la matière vivante plus ou moins différenciée dans ses parties constituantes. L'écrevisse peut encore faire repousser un membre perdu par accident, mais un membre séparé du corps ne peut plus refaire l'individu et, chez les vertébrés supérieurs, on ne connaît plus d'exemple de reconstitution spontanée d'un membre perdu.

A mesure que progresse la différenciation du tissu nerveux et la centralisation locale de son organe dominant, du cerveau, l'individualisation de l'unité de la matière vivante "animal " devient de plus en plus accusée; mais cette réserve faite, on peut dire qu'à partir des cœlentérés, les animaux ne se reproduisent spontanément que par des cellules sexuelles et peuvent être considérés comme des individus proprement dits.

Quelle est alors l'évolution de l'individu véritable et quel est le rôle de cette unité "matière-énergie " indivisible dans l'évolution progressive?

A travers les variations infinies, quant à la durée de la vie, les détails de forme, le mode de reproduction, la nature des éléments de travail et de structure, etc., l'évolution de l'individu animal présente une uniformité très grande et subit les mêmes lois générales que la couche des cellules génératrices d'un végétal.

Il y a pourtant une différence qu'il est important de signaler: les cellules de la couche génératrice d'un végétal qui deviennent somatiques, peuvent se multiplier, ainsi que nous l'avons vu, à l'infini, tan- 
dis que chez un animal bien individualisé, chez un vertébré supérieur, ces cellules ne se multiplient que pendant la vie embryonnaire, à l'exception toutefois des éléments figurés du sang (leucocytes et hématies) de certaines cellules épithéliales et des cellules sexuelles. Une multiplication trop rapide des cellules épithéliales ou conjonctives est toujours pathologique: (cancer). A partir de la naissance de l'individu, toutes les autres cellules qui le composent s'accroissent, se modifient, se différencient, mais ne se multiplient pas.

En étudiant l'évolution de certains tissus, par exemple du tissu musculaire des oiseaux ou des mammifères les mieux connus, on constate que les fibres molles et gélatineuses chez les tout jeunes sujets prennent peu à peu de la consistance et de la fermeté, grâce à l'épaississement des membranes conjonctives qui enveloppent les faisceaux musculaires; avec l'âge, les muscles peuvent être remplacés, dans certains cas, par des tendons, et ces derniers par des tissus cartilagineux en enfin osseux (1).

Le même phénomène se produit pour tous les autres tissus et organes. Les vaisseaux sanguins deviennent moins souples et moins perméables, les échanges nutritifs, les sécrétions des glandes, la mobilité des muscles, la sensibilité nerveuse, etc., deviennent plus lentes et plus pénibles. L'individu animal vieillit et meurt comme la couche génératrice d'une plante.

L'épaississement progressif des parois cellulaires, ainsi que l'accumulation des substances de plus en plus stables dans les tissus qui constituent les char-

(1) On peut facilement suivre ces remplacements successifs dans les membres des oiseaux. 
pentes, ont pour effet, d'une part, de rendre l'organisme plus résistant et plus vigoureux, d'autre part de ralentir le processus de nutrition et, par conséquent, l'accroissement des cellules.

L'arrêt de cet accroissement des cellules coïncide avec l'entrée de l'organisme dans l'état adulte, mais cet arrêt est loin d'être simultané pour les cellules de tous les tissus, organes et glandes d'un organisme pluricellulaire différencié.

On sait que le thymus atteint, chez l'homme, son plus haut degré de développement à l'âge de deux ans et qu'il dégénère ensuite peu à peu en tissu adipeux, pour disparaître complètement, en tant que glande, à l'âge de 10 à 12 ans. Les organes de la reproduction ne commencent à fonctionner normalement qu'à l'âge de 12 à 15 ans (plus tôt dans les pays chauds) et s'atrophient, chez la femme, entre 45 à 55 ans; chez l'homme beaucoup plus tard. On peut obtenir un accroissement des cellules musculaires à un âge déjà assez avancé, par des massages ou une gymnastique appropriée, et on peut en arrêter le développement chez l'enfant, par un travail trop pénible et trop prolongé. Dans ce dernier cas, on obtient même un arrêt prématuré du développement de tout l'organisme. C'est la cellule nerveuse qui semble résister le plus longtemps à l'envahissement par les substances de structure. Elle fonctionne souvent encore d'une façon normale et même plus active que dans la jeunesse, quand le reste de l'organisme manifeste déjà des signes évidents de fatigue.

L'évolution des différents tissus ou organes peut donc être plus ou moins précoce ou tardive, lente ou rapide, suivant la somme et la nature du travail spontané ou imposé à chaque organe ou tissu. Il est bien 
difficile de fixer une limite entre l'enfance et l'âge adulte. La maturation des organes de la reproduction pourrait servir d'étape entre ces deux phases d'évolution (parce qu'elle se manifeste d'une façon assez brusque chez chaque individu et assez uniforme chez tous les individus de la mème espèce) si ce phénomène coïncidait avec l'arrêt de croissance de l'organisme, ce qui n'est jamais le cas, du moins chez les animaux supérieurs.

Il est encore plus difficile d'établir une séparation biẹn nette entre l'état adulte et la vieillesse, qui arrive progressivement par étapes dont la dernière est marquée, chez l'homme, par un tassement de la charpente osseuse résultant de l'aplatissement et du durcissement des rondelles eartilagineuses qui séparent les vertèbres.

La cause en est la même que celle qui a déterminé l'accroissement dans l'enfance et dans la jeunesse, ainsi que la pleine force dans l'âge mûr. Les éléments de structure se développent progressivement et, arrivés à un certain maximum, ils subissent des transformations qui les rendent plus compacts, mais, en même temps, plus friables et, par-conséquent, moins résistants. En outre, ils s'incrustent de sels (oxalates, phosphates, urates de calcium, etc.), de sorte que le métaboliøme intra et intercellulaire, ainsi que les mouvements volontaires ou réflexes deviennent de plus en plus lents, difficiles et pénibles; " on perd l'envie et la volonté de se mouvoir ".

Les réactions, devenant plus lentes, dégagent de moins en moins d'énergie calorique et l'abaissement général de température réagit à son tour sur certaines combinaisons, qui deviennent encore plus lentes, pour s'arrêter complètement à un moment donné. 
Tout concourt à la fois pour arrêter les mouvements d'ensemble et de détail, volontaires ou réflexes, qui constituent la vie d'un organisme complexe. Mouvements, qui sont déterminés par le jeu des réactions intracellulaires et qui, à leur tour, provoquent des réactions et les entretiennent.

Si l'homme pouvait vivre à l'abri de tout accident, dans un milieu stérile, il finirait son évolution dans. une immobilité extérieure et intérieure complète, déterminée par l'arrêt des réactions indispensables à la nutrition de ses cellules.

Il ne faudrait pourtant pas en conclure que toutes les cellules qui composent un organisme animal doivent, ou devraient nécessairement mourir en même temps que l'ensemble qui forme l'individu.

Carrel a montré que la cellule conjonctive peut vivre et se multiplier indéfiniment quand on l'isole de l'organisme et quand on la place dans un milieu nutritif convenable, on doit donc en déduire que ce ne sont pas les facteurs du milieu extérieur, mais bien certains facteurs de son milieu intérieur qui déterminent l'évolution cyclique de l'individu animal.

Quels peuvent être alors ces facteurs du milieu intérieur qui déterminent le vieillissement et la mort de l'animal?

Metchnikoff, un des biologistes les plus éminents de ces temps derniers, a consacré les dix à quinze dernières années de sa vie à l'étude de ce problème et s'il ne l'a pas complètement résolu, il en a indiqué les éléments de la façon claire, précise et documentée qui caractérise tous ses travaux.

Après avoir constaté et prouvé, tout d'abord, qu'il y a dans tout organisme animal des cellules spéciales: les gros leucocytes mononucléaires, les cel- 
lules fixes endothéliales (qui tapissent les parois intérieures des vaisseaux sanguins), les cellules névrogliques du cerveau, et sarcoplasmiques des muscles, qui détruisent toute substance digestible étrangère introduite dans l'organisme ou tout tissu de son milieu intérieur, qui a cessé de fonctionner normalement, et ensuite, que ce sont précisément les mêmes cellules appelées phagocytes macrophages qui, dans les cas pathologiques, se mettent à la place des tissus actifs détruits et les transforment en tissu conjonctif,

Metchnikoff a cherché à démontrer que le vieillissement de l'individu est l'effet d'un processus identique.

Le mécanisme du vieillissement serait donc l'atrophie de certains organes essentiels, qui entraîne naturellement une rupture de l'équilibre fonctionnel et un état de moindre résistance de l'organisme tout entier.

Ensuite, en cherchant à expliquer pourquoi les macrophages qui vivaient jusque là en parfaite harmonie avec les cellules de tous les autres tissus, se mettent, à un moment donné, à en dévorer quelquesactifs détruits et les transforment en tissu conjonctif. Metchnikoff a trouvé, que la première cause de l'apparition de cette chimiotaxie positive ou du changement d'une chimiotaxie négative en positive ne pouvait être autre qu'une intoxication et, par là, une paralysie fonctionnelle plus ou moins totale des cellules attaquées.

Parfois, un simple manque d'exercice habituel d'un organe peut amener cet état de moindre résistance, et on dirait, que les phagocytes ne font que guetter Yoccasion de se jeter sur les cellules qui ne fonctionnent pas normalement et les digérer. 
Ainsi, par exemple, on sait que chez le protée (1), qui vit à l'abri de la lumière, l'œil est envahi par les phagocytes qui finissent par transformer le cristallin et le corps vitré en tissu conjonctif.

La cause de l'apparition de cette chimiotaxie positive, qui attire les phagocytes aux tissus en état de moindre résistance, ne serait pas physiologique, elle serait, d'après Metchnikoff, toujours déterminée par une intoxication dans le cas d'atrophie sénile, par les maladies infectieuses aiguës, ou bien, le plus souvent, par les excrétions des microbes qui pullulent toujours dans le gros intestin. Ces microbes produisent toujours, entre autres substances, de l'indol dont l'action destructive sur certains organes a été démontrée par expérience.

Ainsi, en ajoutant pendant plusieurs mois, un peu d'indol à la nourriture ordinaire des cobayes et des lapins, Metchnikoff a constaté chez ces animaux des lésions sclérotiques de l'aorte et une hypertrophie du tissu fibreux dans le foie et dans les reins.

Ces observations et expériences sont certainement très probantes. Il n'est pas douteux que les maladies infectieuses, ainsi que les microbes de la flore intestinale peuvent provoquer des lésions cellulaires suivies d'une intervention néfaste des phagocytes, mais s'ensuit-il nécessairement que si l'intestin d'un animal était complètement dépourvu de microbes et si l'animal échappait à toute intoxication ou infection, il pourrait rester éternellement jeune?

Tous les autres vertébrés, en dehors des mammifères, ne possèdent pas de gros intestin et n'ont qu'une flore intestinale très pauvre; les intestins

(1) Petit batracien que l'on trouve dans les lacs souterrains de la Dalmatie. 
des larves de certains insectes ne contiennent pas de microbes du tout, et pourtant tous ces animaux finissent toujours par vieillir et mourir.

Il y a là un malentendu; la question de l'évolution cyclique ou indéfinie de l'individu doit être posée d'une autre façon, et notamment: l'organisme le plus individualisé actuellement, l'homme, pourrait-il ne jamais vieillir si, étant né sans aucune tare héréditaire, il échappait dans le cours de sa vie, à toute infection ou intoxication, ou à un facteur destructif quelconque, venant du milieu extérieur, ou, en d'autres termes, si son milieu intérieur, tel qu'il est constitué aujourd'hui, ne porte pas en lui, de par sa composition et sa structure, les causes nécessaires de son vieillissement et de sa mort.

Nous croyons pouvoir affirmer que l'homme tel qu'il est constitué aujourd'hui et disposant de l'ensemble de ses connaissances actuelles, tant en ce qui concerne la composition, la structure et le fonctionnement de son milieu intérieur, que les facteurs du milieu extérieur qu'il peut mettre en jeu pour influencer la structure et les fonctions de ses organes, ne peut pas vivre au delà d'un certain âge, qu'il doit nécessairement vieillir et mourir par le seul jeu physiologique des réactions, dont il est le siège.

Même en menant la vie la plus normale, en se nourrissant des substances les plus appropriées à son équilibre physico-chimique et physiologique, en entretenant par une gymnastique modérée ses tissus et organes dans la meilleure forme possible, un homme vieillirait parce que, dans la série des réactions chimiques qu'il subit, il se forme toujours des composés stables et insolubles qui s'accumulent progressivement et rendent toujours, à un moment 
donné, le métabolisme intercellulaire et interorganique impossible.

Mais alors, une autre question se pose : Peut-on raisonnablement affirmer qu'il en sera toujours ainsi? - Certainement non! - Nous ne savons pas encore intervenir utilement pour prolonger la durée de l'état de réelle jeunesse ou de l'âge mûr; mais les découvertes concernant les fonctions et le rôle des glandes à sécrétions internes, ainsi que l'action de certaines substances organiques et inorganiques sur ces glandes et sur les centres nerveux, aidées par l'exercice soutenu de l'intelligence et de la volonté, nous permettront certainement un jour peut-être pas trop éloigné - d'arrêter la formation ou l'accumulation de ces substances insolubles qui " encrassent" nos organes et nos filtres, tout en conservant l'équilibre d'ensemble nécessaire pour une vie normale, dont la durée dépendra de notre vơlonté.

\section{1. - Evolution des espèces animales}

Il s'agit maintenant de comprendre comment un être unicellulaire a pu devenir homme, et de donner à ce phénomène le sens biologique le plus vraisemblable.

Pour rendre cet exposé aussi clair que possible, il faut donc se rappeler que la succession de la formation des organismes de plus en plus complexes. et différenciés dans les temps géologiques, ainsi que le développement de l'organisme le plus individualisé, d'une simple cellule, d'un ovule fécondé, sont des faits constatés et non des simples vues de l'esprit. 
Ceci bien admis, nous constatons encore aujourd'hui l'existence simultanée des organismes à tous les degrés de l'évolution: des êtres unicellulaires, peutêtre même unimicellaires (microbes indivisibles) et, en même temps, d'innombrables espèces de végétaux et d'animaux inférieurs et supérieurs, qui ont eu un point de départ à un certain niveau de l'évolution progressive, et n'ont pas dépassé ce niveau depuis qu'ils existent. Nous constatons donc, en un mot, qu'un très grand nombre d'organismes n'ont pas subi d'évolution progressive, qu'ils sont restés semblables à eux-mêmes depuis les temps les plus reculés et nous devons en déduire, nécessairement, que si les individus peuvent varier, l'espèce - prise dans son ensemble - peut être d'une stabilité à toute épreuve.

Nous avons constaté un phénomène analogue dans l'évolution de la matière minérale : l'existence simultanée des électrons, des atomes libres, des molécules, des micelles, et nous avons vu qu'en chimie-physique on explique ce fait par une loi d'équilibre qui s'oppose à la combinaison en unités plus complexes de toutes les unités plus simples, réunies dans un certain milieu; qu'en mélangeant par exemple une certaine quantité d'acide chlorhydrique et de soude caustique, en proportions exactement équivalentes, tous les atomes du chlore ne se combineront pas avec tous ceux du sodium, qu'il restera toujours dans le liquide un certain nombre de ces atomes libres.

Il existe peut-être une loi d'équilibre général, applicable à l'évolution et aux rapports de toutes les unités " matière-énergie " sur une planète ou dans l'univers, mais la loi physico-chimique à laquelle nous venons faire allusion, ne joue certainement aucun rôle dans les rapports entre les unités vivantes. 
Le fait qu'il y a encore aujourd'hui des microbes et des infusoires qui mènent une existence libre et à peu près toujours la même, et que d'autres individus de la même espèce originelle ont évolué jusqu'à l'homme, de même que l'existence des électrons et des atomes à côté des molécules, à certainement pour cause les variations locales temporaires du milieu extérieur. Si tous les atomes d'oxygène et d'hydrogène qui existaient à un moment donné, dans l'atmosphère terrestre, ne se sont pas combinés en même temps en molécules d'eau, sous certaines conditions de température et de pression, c'est certainement surtout parce que ces conditions n'étaient pas les mêmes partout, comme aujourd'hui encore les étincelles électriques provoquent la formation de l'eau, là seulement où elles se produisent.

Ce sont également les conditions très différentes des milieux dans lesquels les individus de la même espèce ont pu se trouver pendant un temps suffisamment long, qui ont provoqué des changements durables dans la constitution et les fonctions de leur milieu intérieur.

Et, s'il existe une loi d'équilibre général, qui règle l'évolution des unités de la matière vivante et les rapports entre celles de ces unités qui forment les échelons successifs de l'évolution progressive et celles qui restent en route et gardent indéfiniment l'ensemble des caractères spécifiques, comme autant de branches collatérales de cette échelle ascendante sans fin, comme autant de témoins de l'évolution progressive, cette loi n'intervient pas directement dans les modifications des organismes, mais indirectement, en équilibrant d'une certaine façon les variations des facteurs du milieu extérieur. 
Il résulte donc de ce qui précède, que l'évolution des espèces, ou la formation des espèces nouvelles, plus élevées parce que plus différenciées, est la résultante de deux facteurs antagonistes: $1^{\circ}$ les variations du milieu extérieur, qui peut amener des modifications favorables à une évolution ascendante, $2^{\circ}$ la fixité ou la stabilité des caractères spécifiques, transmis par l'hérédité, qui fait que l'œuf du brochet donne toujours naissance à un brochet et l'œuf de l'homme à l'homme.

Cette constance de caractères spécifiques a pour cause primordiale la "mémoire " du plasma vivant, que nous avons comparée à la force d'inertie d'un tourbillon ou d'un giroscope qui, animé d'une certaine vitesse, et pourvu d'une certaine ampleur, permet à ce tourbillon de résister aux facteurs extérieurs d'une vitesse et d'une puissance moindres, et communique son propre mouvement aux substances qu'il entraîne dans son milieu intérieur.

On a constaté aussi, que cette mémoire ou force d'inertie de la matiène vivante devient d'autant plus puissante qu'elle s'exerce, d'une part, plus longtemps dans les mêmes conditions (on se rappelle un fait qui s'est produit une fois, mais on se le rappellera d'autant mieux et d'autant plus longtemps, qu'il se reproduira plus souvent); d'autre part, qu'elle se produit dans une unité d'inertie elle-même plus puissante, plus complexe et plus fortement individualisée. Chez l'homme, les caractères spécifiques sont plus stables et plus durables que chez le poisson et, chez ce dernier, plus stables que chez un protozoaire, bien que l'espèce de ce dernier soit beaucoup plus ancienne que celle du poisson.

Et si, chez les microbes, on peut obtenir des 
variétés ou des races nouvelles en quelques mois, tandis que chez l'homme, on n'a pas observé de modifications de race appréciables depuis les temps historiques, c'est qu'il faut au moins cent mille fois plus de temps pour obtenir le même nombre de générations chez l'homme que chez les microbes, et il faut encore tenir compte des différences très grandes de la force d'individualisation dans les deux cas, ainsi que du fait que chez les microbes le caractère acquis par une cellule est intégralement transmis aux deux cellules filles, tandis que chez les organismes qui se multiplient exclusivement par la conjugaison de deux cellules sexuelles, les caractères acquis par un des parents, peuvent être annulés ou modifiés par ceux de l'autre.

Ainsi, en tenant compte de tous les éléments actuellement connus, qui constituent le problème de l'évolution progressive des espèces animales et notamment des modifications que peuvent provoquer les variations des facteurs du milieu extérieur, de la stabilité des caractères spécifiques, du degré de l'individualisation des unités d'une espèce et de la durée des révolutions individuelles, on peut se représenter la filiation de ces espèces, depuis l'organisme unicellulaire jusqu'à l'homme, de la façon suivante :

\section{2. - Filiation des espèces animales}

L'ancêtre ou les ancêtres unicellulaires de tous les animaux devaient avoir la faculté d'assimiler d'abord les produits encore assez complexes de la décomposition des substances albuminoïdes, et ensuite d'opérer eux-mêmes cette décomposition par 
des ferments élaborés dans leur milieu intérieur et excrétés à l'extérieur.

Ces protozoaires, qui se sont distingués ainsi, par leur genre de nutrition, des protistes qui sont devenus végétaux, devaient se multiplier par bipartition, mais aussi par conjugaison, et le premier pas en avant devait consister très probablement dans la formation d'une race, dont les unités se reproduisaient de préférence par conjugaison. Nous avons $\mathrm{vu}$, en effet, que la conjugaison résulte et a pour effet un certain degré d'adaptation à un milieu nouveau et favorise ainsi la formation des races plus résistantes aux variations de ce milieu.

Un tel organisme unicellulaire pouvait encore donner naissance aux végétaux parasites et, en particulier, à la classe des champignons, et nous avons vu que le pas décisif dans la direction de l'embranchement animal, devait être la formation d'un organisme pluricellulaire, muni d'une ouverture conduisant à une cavité intérieure (gastrula) et dont les membranes cellulaires étaient dépourvues de cellulose.

Cet organisme devait se reproduire par des cellules sexuelles spéciales, qui se conjuguaient en œufs. Il était mobile, pouvait changer de milieu ou être transporté d'un milieu à un autre, son évolution individuelle était cyclique et la durée de sa vie individuelle devait être plus longue que celle d'un être unicellulaire se multipliant exclusivement par division.

Soumis à des influences de milieu différentes, en fait de nutrition, température, etc., les individus de cette espèce devenaient différents et transmettaient les nouveaux caractères acquis à leurs cellules sexuelles et à leurs œufs, qui donnaient naissance à 
des individus pourvus de ces caractères nouveaux.

Les caractères acquis se transmettaient ainsi d'œuf en œuf pour lequel le reste de l'organisme, l'ensemble des cellules somatiques n'était qu'un milieu de culture perfectible, parce que à mesure qu'il se différenciait, il devenait de plus en plus sensible à tous les facteurs du milieu extérieur, et faisait profiter de cette différenciation progressive les cellules sexuelles qu'il cultivait.

Les choses se passent encore ainsi aujourd'hui.

Au point de vue de sa signification, de sa raison d'être biologique, l'individu animal (y compris l'homme) ne représente donc qu'une forme d'évolution passagère, un milieu de culture pour les cellules sexuelles, auquel pourtant, ces cellules sont reliées d'une façon beaucoup plus étroite, que ne le sont les êtres unicellulaires libres, à leur milieu de culture extérieur.

\section{3. - Conditions de développement des cellules sexuelles}

Seules les cellules sexuelles et les œufs qu'elles forment, et qui se multiplient par bipartition, sont les unités vivantes infiniment durables, comme les. êtres unicellulaires libres; mais nous avons vu aussi que c'est par la perfectibilité de leur milieu immédiat, de l'individu animal, qu'ils ont pu subir et subiront dans l'avenir les transformations de l'évolution progressive, parce que, seule, une différenciation de plus en plus délicate pouvait leur assurer cette évolution, et qu'une telle différenciation n'est possible que dans un organisme pluricellulaire. 
On peut citer, à ce propos, deux expériences très suggestives de Driesch et de J. Loeb.

Driesch (Zeitschr. für wissensch. Zoologie, t. L, $I I I$, L. V) a constaté qu'en secouant un œuf d'oursin au stade de deux et même de quatre cellules, on peut les séparer et que chacune d'elle donne naissance à un embryon complet, qui ne diffère de l'embryon normal que par une taille plus petite.

J. Loeb (La conception mécanique de la vie, Félix Alcan, Paris) a observé qu'en faisant éclater la membrane d'un œuf d'oursin par l'addition de 100 p. 100 d'eau distillée à l'eau de mer normale, une partie du protoplasma en sort sous forme d'une ou de plusieurs goutelettes qui, toutes, donnent naissance à des embryons complets, normaux.

Les expériences faites dans le but de montrer que dans un œuf fécondé, naturellement ou artificiellement, les substances formatrices sont réparties de façon qu'une portion (la moitié, le quart, ou encore moins) en contient tout ce qui est nécessaire pour la construction de l'organisme entier, conduisent à une suggestion encore plus intéressante que les deux auteurs n'ont pas suffisamment mise en lumière ; elles indiquent. en effet, que les cellules filles de la première et de la deuxième génération peuvent vivre librement, et que, si on continuait à les séparer ainsi à chaque division, on finirait par faire revenir l'œuf d'oursin et, par conséquent, ce qui deviendrait l'oursin lui-même, à l'état d'un être unicellulaire, se multipliant par bipartition.

L'expérience réussirait peut être plus facilement avec les œufs d'un cœlentéré ou d'un porifère. 
En sera-t-il toujours ainsi ? l'homme sera-t-il toujours réduit à obéir passivement à ses instincts, au rôle purement biologique du milieu de culture de son oosperme; sera-t-il toujours condamné aux travaux forcés comme c'est encore le cas aujourd'hui pour l'immense majorité des hommes: à assurer sa subsistance par des travaux pénibles et obligatoires, qui nuisent à son développement individuel et n'ont d'autre but, que de faire vivre ses gamètes et former un nouvel oosperme?

Gertainement non !

L'étude de l'évolution du tissu nerveux nous a montré que l'homme peut prétendre à un avenir meilleur, plus conforme au perfectionnement de son córps et de ses énergies, un avenir qu'il pressent, auquel il aspire déjà et qu'il peut considérer comme réalisable.

Pour comprendre comment on peut y arriver, pour trouver les jalons de la direction de la route qui mènera l'humanité le plus sûrement à cet état d'existence supérieure, plus conforme aux aspirations de l'esprit, il nous faut entrer encore dans quelques détails de structure et de développement de l'individu issu de ses gamètes et de l'oosperme.

\section{4. - Les phases de l'évolution}

Rappelons-nous que, représenté d'une façon schématique, le corps de l'homme se compose de cinq appareils distincts .

$1^{\circ}$ L'appareil de structure et de locomotion: les 
tissus osseux, cartilagineux, fibreux, conjonctifs et musculaires;

$2^{\circ}$ L'appareil de nutrition, composé d'organes de respiration, de digestion, d'assimilation et d'élimination: tube digestif avec ses glandes annexes, glandes salivaires, foie et pancréas, poumons, vaisseaux sanguins et lymphatiques, reins ;

$3^{\circ}$ Système de glandes à sécrétions internes : glandes thyroïdes, hypohyse, capsules surrénales, rate, moelle osseuse, foie, pancréas, glande interstitielle chez l'homme, corps jaune chez la femme, le cerveau;

$4^{\circ}$ Système nerveux central et organes des sens;

$5^{\circ}$ Appareils et organes de reproduction.

Les modifications subies par tous ces appareils pendant la vie de l'individu peuvent influencer l'évolution de ses gamètes et de l'oosperme pendant sa vie intra-utérine, mais l'importance du rôle de ces appareils dans les fonctions essentielles de l'organisme est loin d'être la même, et les répercussions que ces modifications peuvent avoir sur les descendants, dépendent non seulement de la nature des modifications subies par l'individu dans le cours de sa vie, mais aussi et même surtout du moment où elles se produisent, par rapport au degré de développement des gamètes et de l'oosperme.

Il est certain, en effet, qu'un membre cassé ou un accident quelconque arrivé aux appareils de structure et de locomotion, guéri dans de bonnes conditions, ne deviendra jamais un caractère nouveau, directement héréditaire, mais que, par contre, un trouble fonctionnel du système thyroïdien, de l'hypophyse ou du cerveau aura une action plus ou moins immédiate et profonde sur les descendants, 
suivant qu'il coïncidera avec un état de développement plus ou moins avancé des gamètes ou de l'oosperme.

Nous ne savons encore rien de précis sur la nature des réactions qu'un certain facteur du milieu extérieur, un sel, un antigène, une énergie rayonnante ou psychique, produira en dernière analyse sur les cellules sexuelles, et par quels caractères cette réaction se traduira chez les enfants nés de ces cellules; mais nous savons que, pour arriver aux cellules sexuelles, les facteurs du milieu extérieur général doivent passer par une série d'intermédiaires du milieu extérieur immédiat de ces cellules, de l'organisme, et qu'à chacun de ces passages ils peuvent être modifiés quant à la nature des réactions provoquées; que les mêmes tacteurs peuvent produire des effets différents chez des individus qui ne présentent pas le même équilibre fonctionnel.

Nous avons vu que le développement du cerveau dépend des sécrétions des glandes thyroïdes, que la croissance des membres est sous la dépendance de l'hypophyse, que les capsules surrénales règlent, dans une certaine mesure, la circulation sanguine, et nous devons supposer que le fonctionnement, plus ou moins normal et bien équilibré de ces glandes, se répercute sur la structure, la composition et, par conséquent, sur le développement des cellules sexuelles.

Nous savons encore que tout facteur extérieur ne peut provoquer dans l'organisme une modification d'équilibre durable, qu'à la condition de faire apparaître une réaction nouvelle, durable, dans une ou plusieurs de ces glandes à sécrétions internes.

Pour arriver à connaître quelles peuvent être les 
modifications apportées à l'hérédité par les changements du milieu extérieur général, il faudrait donc étudier en premier lieu l'action de différents facteurs de ce milieu, sur l'organisme de l'individu. Ensuite, il faudrait considérer la répercussion de chacune de ces réactions particulières sur ses descendants, et surtout poursuivre ces observations et expériences pendant une assez longue série de générations, parce que, autant qu'il a été possible de s'en rendre compte actuellement, si l'apparition d'un caractère nouveau chez un individu est généralement brusique, elle est certainement la conséquence d'une longue filière de réactions qui sont passées inaperçues.

C'est là précisément que réside la difficulté du problème qu'il s'agit de résoudre. Dans les expériences faites jusqu'à présent, soit en agissant directement sur l'œuf ou sur l'embryon, soit sur les cellules sexuelles, par l'intermédiaire de l'organisme qui les porte, on a cherché à obtenir des résultats immédiats et on a obtenu des monstres. Par ces interventions, généralement trop brutales, on a montré qu'il est possible de faire apparaître chez l'individu des caractères nouveaux, de rompre ainsi l'équilibre héréditaire individuel, mais non pas de vaincre une fois pour toutes la force d'inertie héréditaire, qui fait revenir chaque organisme vivant et capable de vivre, à son équilibre antérieur. Ce n'est pas en procédant de cette façon que l'on obtiendra des races ou des espèces nouvelles, durables.

Les races améliorées de plantes cultivées et d'animaux domestiques obtenues avec beaucoup de peine, dégénèrent rapidement, ou reviennent à l'état primitif, si on ne fait pas intervenir les mêmes facteurs d'amélioration d'une façon continue. 
On peut dire qu'en principe, une modification durable, qui pourrait devenir héréditaire, ne peut être obtenue que par des interventions stimulantes, en doses stimulantes, quelle que soit la nature du facteur agissant et de l'organe sur lequel il agit. La rupture de l'équilibre de l'organe sur lequel on agit, doit être suivie de l'établissement d'un équilibre fonctionnel général, et c'est alors seulement que cet état d'équilibre général, entretenu pendant un certain nombre de générations, peut agir d'une façon décisive et durable sur les cellules sexuelles ainsi que sur l'oosperme.

Mais, en réalité, ce qui nous intéresse ici, ce que nous voudrions faire ressortir, ce n'est pas de rechercher si et comment on peut obtenir une modification individuelle ou héréditaire quelconque; ce qu'il s'agit de rechercher ce sont "le " ou "les" facteurs qui avaient été et seront dans l'avenir les causes déterminantes de l'évolution progressive, de la formation de races et d'espèces dont les individus seraient de plus en plus différenciés, de plus en plus capables de dominer les facteurs du milieu extérieur général, de les adapter à un certain développement, dans une direction déterminée de l'individu et, par là, de ses cellules sexuelles.

Or, nous en avons vu (chap. 28) que le facteur déterminant de l'évolution progressive est le développement de plus en plus considérable en volume, suivi d'une structure de plus en plus complexe et d'une différenciation de plus en plus variée et délicate de l'encéphale et, en particulier, du pallium.

Nous avons vu aussi que les sécrétions des glandes thyroïdes et de l'hypophyse exercent une action profonde sur le développement général, et en particulier 
sur celui de l'encéphale. L'hypertrophie du système thyroïdien a pour conséquence un état pathologique qui se traduit par la formation d'un goître; l'insuffisance fonctionnelle de ces glandes chez l'enfant provoque un arrêt dans le développement du cerveau qui se traduit par le crétinisme. L'insuffisance fonctionnelle de l'hypophyse a pour conséquence l'infantilisme, son' hypertrophie, un gigantisme disharmonieux, accompagné de débilité physique et psychique.

Et il est certain que les réactions qui conduisent à ces effets apparents ne sont pas simples, ne résultent pas exclusivement de la quantité trop grande ou trop petite de thyroïdine ou de l'hypophysine sécrétée, mais bien plutôt d'une rupture de l'équilibre général, dans les fonctions de toutes les glandes endocrines, des rapports entre les quantités et de la nature de toutes les substances autocoïdes (1) et des harmozones (2) versées dans la circulation.

Le chemin à suivre pour l'étude des facteurs positifs de l'évolution progressive est donc assez nettement indiqué dès aujourd'hui: ce serait de préciser de mieux en miuex le rôle des glandes endocrines dans le développefent du pallium chez l'individu, et celui de l'action des facteurs du milieu extérieur sur le développement et les fonctions de ces glandes, et enfin l'action des uns et des autres sur-les gamètes et l'oosperme à l'état d'évolution embryonnaire.

Pour compléter cette étude, il nous faut donc encore examiner comment et à quel moment les cellules sexuelles peuvent être influencées dans leur développement, par leur milieu immédiat, c'est-à-dire par

(1) Sécrétions régulatrices stimulantes ou empecchantes des glandes endocrines. (Schafer.)

(2) Sécrétions formatrices des glandes endocrines. (Gley.) 
l'individu qui les porte, et pour ce faire, il est indispensable d'entrer dans quelques détails de structure et de développement des gamètes et de l'oosperme.

\section{5. - Fécondation}

On ne connaît encore les détails du développement de l'oosperme (ovule fécondé par le spermatozoïde), que chez un petit nombre d'animaux (vers, tuniciers, batraciens, oiseaux), mais, de ce qu'on sait, on peut schématiser ce développement de la façon suivante:

Structure. - L'ovule ou la gamète femelle, relativement gros (quelques centièmes à quelques dixièmes de millim., chez la femme, 0,2 de millim.), est rond et immobile. C'est une cellule composée d'un noyau et d'un plasma, entouré d'une membrane très mince.

Le spermatozoïde, ou gamète mâle, généralement plus petit que le noyau de l'ovule, est une cellule mobile, presque complètement dépourvue de cytoplasme, qui a généralement la forme d'un têtard muni d'une arrière-tête et d'une queue relativement très longue. La tête, c'est le noyau du spermatozoïde, l'arrière-tête contient généralement un petit corpuscule rond, le centrosome.

L'acte de fécondation consiste dans la pénétration de la tête et de l'arrière-tête du spermatozoïde dans l'intérieur de l'ovule, la queue reste en dehors et disparaît.

Il y a toujours beaucoup de spermatozoïdes qui tourbillonnent autour d'un ovule, mais un seul a le droit d'y pénétrer et, pour qu'il y pénètre, il faut que l'ovule lui fasse une avance. A l'approche de l'élément mâle, la cellule femelle gonfle sa membrane

DAxysz. - La genèse de l'énergie psychique. 
en un " cône de fécondation ", et c'est dans le sommet de ce cône que s'enfonce la tête du spermatozoïde. Ceci fait, l'ovule ne se laisse pénétrer par aueun autre spermatozoïde, ou du moins il ne fait plus d'avance à aucun. C'est ainsi que se forme l'oosperme et que commence le développement d'un individu nouveau.

\section{6. - Evolution de l'oosperme. Division et différenciation}

L'ovule fécondé se divise comme toute autre cellule. Les noyaux du spermatozoïde et de l'ovule, formés de filaments enroulés en pelotes, se placent visà-vis l'un de l'autre et le filament du noyau mâle se soude par un bout à un bout du filament femelle, - pour former un seul filament appelé spirème qui se déroule et se divise en fragments appelés chromosomes, plus ou moins nombreux suivant les espèces. Alors chaque fragment mâle s'accole à un fragment femelle sans qu'il y ait fusion complète entre eux ; ils augmentent alors de volume, se divisent en deux, les deux paires ainsi formées se séparent, et les paires ainsi séparées se réunissent en deux groupes égaux qui s'éloignent l'un de l'autre et forment ainsi les noyaux de deux cellules filles qui résulteront de la division de l'oosperme.

Pour chaque espèce d'animaux, le nombre de chromosomes est constant. Les noyaux des gamètes de l'Ascaris mélagaloçephala (un ver parasite) contiennent chacune deux chromosomes, et le processus de fécondation, ainsi que de la division de l'oosperme peuvent être facilement représentés par des lettres sans avoir besoin de recourir à des dessins. 
En appelant $\mathbf{S}^{\mathbf{a}}$ et $\mathbf{S}^{\mathrm{b}}$ les deux chromosomes du spermatozoïde, et $0^{a}, O^{b}$ ceux de l'ovule, nous aurons donc d'abord une chaîne :

$$
\mathrm{S}^{\mathrm{a}}+\mathrm{S}^{\mathrm{b}}+\mathrm{O}^{\mathrm{a}}+\mathrm{O}^{\mathrm{b}}
$$

ensuite, division en quatre chromosomes distincts :

Chromosomes: $\mathrm{S}^{\mathrm{a}}, \mathrm{S}^{\mathrm{b}}, \mathrm{O}^{\mathrm{a}}, \mathrm{O}^{\mathrm{b}}$

puis un groupement en deux paires de : mâle et femelle :

$$
\begin{aligned}
& \mathrm{S}^{\mathrm{a}} \text { et } \mathrm{S}^{\mathrm{b}} \\
& \mathrm{O}^{\mathrm{a}} \text { et } \mathrm{O}^{\mathrm{b}}
\end{aligned}
$$

ensuite, chaque chromosome se divisant en deux, on aura quatre paires, soit:

$$
\begin{aligned}
& \mathrm{S}^{\mathrm{a}}, \mathrm{S}^{\mathrm{a} \prime} \text { et } \mathbf{S}^{\mathrm{b}}, \mathbf{S}^{\mathrm{b}} \\
& \mathrm{O}^{\mathrm{a}}, \mathrm{O}^{\mathrm{a} \prime} \text { et } \mathrm{O}^{\mathrm{b}}, \mathrm{O}^{\mathrm{b}}
\end{aligned}
$$

qui se réuniront en deux noyaux distincts:

$$
\mathrm{S}^{\mathrm{a}}+\mathrm{S}^{\mathrm{a}}+\mathrm{O}^{\mathrm{a}}+\mathrm{O}^{\mathrm{a} \prime} \text { et } \mathrm{S}^{\mathrm{b}}+\mathrm{S}^{\mathrm{b}}+\mathrm{O}^{\mathrm{b}}+\mathrm{O}^{\mathrm{b}}
$$

dans chacun desquels il y aura quatre chromosomes dont deux mâles et deux femelles.

Cette mise en scène, dont les détails sont bien plus compliqués et plus difficiles à suivre quand le nombre des chromosomes est plus grand (il y en a 24 chez l'homme) est dirigée par deux corspuscules très petits appelés centrosomes, un pour chaque gamète, qui apparaissent aussitôt après l'entrée du spermatozoïde dans l'ovule et qui, aussitôt apparus, provoquent.dans le cytoplasme des courants dans toutes les directions, en rayons droits, dont ils sont les centres. Dans la région où les rayons de deux centrosomes se rencontrent, il forment done un fuseau et ce sont les courants cytoplasmiques établis dans ce 
fuseau, qui provoquent tous les mouvements des chromosomes que nous venons de décrire.

Les divisions successives des cellules filles de l'oosperme se continueront ainsi pendant la vie embryonnaire de l'organisme, de sorte que le noyau de chaque cellule nouvellement formée contiendra toujours le même nombre de chromosomes mâles et femelles qui ne fusionnent jamais. Toutes les cellules de l'organisme adulte et, par conséquent, cet organisme tout entier, mâle ou femelle, est composé pendant toute sa vie, d'un nombre égal d'éléments mâles et femelles.

\section{7. - Relations entre l'individu et ses cellules sexuelles}

A mesure qu'elles se multiplient, les cellules issues de l'oosperme se différencient. Les unes deviendront épiderme ou tissu nerveux, d'autres des muscles, d'autres des glandes, mais cette différenciation ne débute pas chez toutes les espèces, au même stade de division. Nous avons vu que les quatre premières cellules de l'œuf d'oursin peuvent être séparées et donneront chacune un individu normal, complet, chacune d'elle contient donc la même quantité de mêmes substances formatrices. Chez d'autres animaux, par exemple chez une ascidie, la Styela (d'après Conklin), la différenciation commence dans l'oosperme. On ne connaît ces détails que dans bien peu d'espèces et ils n'ont d'ailleurs que peu d'importance pour nous.

Ce qu'il nous importe, par contre, de faire ressortir ici, c'est que les cellules différenciées en un des trois feuillets principaux: ectoderme, mésoderme et endoderme, et en cellules sexuelles nouvelles, peuvent encore se différencier dans le cours du développement 
de l'individu, au sein du même feuillet, en cellules de la même catégorie, mais ne se transforment plus en cellules d'un autre feuillet. Elles peuvent être détruites, comme nous l'avons vu (p. 238) et remplacées par des cellules conjonctives ou fibreuses; une cellule ectodermique peut devenir épidermique, épithéliale, glandulaire ou nerveuse, elle ne peut jamais devenir musculaire ou sexuelle; de même que cette dernière, une fois différenciée, ne peut devenir autre chose qu'un spermatozoïde ou un ovule. Et cette irréversibilité des cellules différenciées provient de ce fait, que déjà, avant toute différenciation apparente, il se produit, dans l'oosperme ou dans ses premières cellules filles, des déplacements des différentes parties du cytoplasme provoqués par les rayonnements des centrosomes, de façon que les deux cellules filles peuvent ne pas contenir les mêmes substances et qu'une fois qu'elles sont séparées et isolées l'une de l'autre par des membranes propres, l'échange des substances albuminoïdes formatrices devient impossible.

Les cellules sexuelles dérivent donc directement de l'oosperme, et leur différenciation est généralement plus précoce, que celle des cellules de tous les autres tissus. Leur mûrissement est, par contre, généralement le plus tardif. Chez la femme, le développement des ovules se poursuit jusqu'au moment de la ménopause; chez l'homme la formation des spermatozoïdes peut durer jusqu'à l'âge le plus avancé, mais il est très probable que toute la réserve des spermatozoïdes et d'ovules en germe, des spermatogonies et des oogonies dont l'individu disposera pendant sa vie, se trouve formée pendant la vie embryonnaire, avant la naissance. 
Ainsi, chez l'homme, les cellules sexuelles, différenciées dès le début de la vie intra-utérine de l'embryon, se multiplient rapidement, par simples bipartitions, et s'accumulent dans les organes sexuels jusqu'à la naissance. A partir de ce moment, elles ne se multiplient plus, elles augmentent de volume (mais pas toutes à la fois) et mûrissent progressivement les unes après les autres.

A partir du moment où elles ont cessé de se multiplier, on a appelé les cellules sexuelles: oocytes et spermatocytes. La maturation des premiers oocytes se manifeste chez les jeunes filles par l'apparition des premières "règles ". Chez les garçons de la même race, le mûrissement des premiers spermatocytes est, en moyenne, un peu plus tardif, mais les uns et les autres subissent encore une transformation avant de donner naissance à des ovules et des spermatozoïdes libres, prêts à la conjugaison et au moment même de cette libération.

Chaque cellule sexuelle se divise rapidement, deux fois de suite, de façon à former quatre cellules filles.

La première division s'opère normalement. Les filaments chromatiques mâle et femelle, du noyau de la première cellule, se fragmentent, chez l'homme en 24 bâtonnets; chacun de ces bâtonnets se fend en deux, la moitié mâle de chaque bâtonnet s'accole à une moitié du bâtonnet femelle. Les 48 paires, ainsi formées, se séparent d'abord et se réunissent ensuite en deux groupes de 24 paires qui constituent les noyaux des deux premières cellules filles.

A la deuxième division, les bâtonnets chromatiques ne se fendent pas en deux. Ils s'accolent en 24 paires et se séparent pour former deux groupes de douze paires, de sorte que chacune des deux cel- 
lules filles de la deuxième division ne contiendra plus que douze chromosomes mâles et autant de femelles qui, par l'acte de fécondation, reformeront de nouveau dans l'oosperme un noyau complet de 24 chromosomes.

Bien que tous ces détails n'étaient pas absolument indispensables à la démonstration des idées que nous poursuivons, nous avons tenu à les reproduire iei, d'après les recherches les plus récentes de Wilson, Boveri, Stevens, Conklin, etc., pour indiquer que la complexité de composition en substances chimiquement différentes des cellules sexuelles et de l'oosperme est beaucoup plus grande qu'on ne le soupçonnait, il y a encore peu d'années.

Il est certain que l'oosperme ne contient pas les rudiments de tous les tissus et organes dont est pourvu l'organisme adulte qui en provient. Il en contient les substances formatrices. Les rudiments des tissus se forment seulement pendant la vie embryonnaire par la multiplication et la différenciation des cellules embryonnaires, qui continueront ensuite à se différencier par l'accroissement, sans se multiplier; mais il est certain aussi que l'oosperme contient toutes les substances formatrices différentes qui formeront ensuite les tissus nerveux musculaires, glandulaires, ainsi que les cellules sexuelles.

Dans les gamètes, ces substances sont d'abord plus ou moins mélangées dans toutes leurs parties; mais dans l'oosperme elles commencent à se grouper, suivant une loi de polarité primordiale, probablement sous l'action des centrosomes, et se répartissent au fur et à mesure des divisions successives, dans des cellules spéciales, ecto, endo et mesodermiques, $e x-$ cepté dans les cellules sexuelles, dans lesquelles tou- 
tes ces substances différentes restent toujours réunies. La seule séparation et différenciation qui s'opère dans ces cellules, et cela probablement autant dans les ovules que dans les spermatozoïdes, est celle des substances qui donneront naissance à la formation des organes et, par là, des individus femelles et mâles.

L'oosperme et les gamètes qui en dérivent, et dont la multiplication doit aboutir à la formation d'un nouvel oosperme d'un organisme quelconque, de l'homme comme d'une plante, doit donc être considéré comme un organisme unicellulaire, qui se multiplie par un certain nombre de bipartitions, coupées périodiquement par des conjugaisons, et pour lequel tout le reste du corps de l'individu, "le soma ", animal ou plante, n'est qu'un milieu de culture toujours perfectible, par lequel l'oosperme subit l'évolution progressive.

Pour cet organisme unicellulaire dont l'évolution a débuté par une micelle albuminoïde et qui a abouti actuellement à l'homme, l'albumen d'une graine, les pétales d'une fleur, le corps de l'animal, la force virile, l'intelligence de l'homme, les charmes, les qualités morales de la femme, ne sont que les éléments d'un milieu spécial de nutrition et de protection pour son développement, de sélection pour l'acte de fécondation.

\section{8. - Transmission de caractères nouveaux}

Maintenant, nous pouvons nous rendre compte comment et à quel moment la partie somatique de l'organisme peut, à la longue, en se modifiant ellemême, transmettre ces modifications aux cellules sexuelles. 
Je ne puis pas partager ehtièrement l'opinion de Conklin, quand il dit (L'hérédité et le milieu, p. 23): "A aucun moment, ni avant, ni après la naissance, la mère n'est pour l'enfant qu'une nourrice. Les influences héréditaires sont transmises exclusivement par la cellule œuf et par la cellule spermatozoïde, et le développement intra-utérin est sans action sur elles " et, p. 28: "Le plasma sanguin passe de l'un à l'autre par un phénomène de filtration, et les seules influences maternelles qui puissent s'encrer sur l'embryon, pendant le cours de son développement, sont celles susceptibles d'être convoyées par le plasma sanguin et qui sont donc, principalement, d'ordre nutritif. "

On pourrait même dire que ces influences sont exclusivement d'ordre nutritif, sans y voir une raison de nier la possibilité des modifications de certains caractères somatiques de l'embryon, et par là, des modifications des gamètes qui se développent dans cet embryon, par le plasma de la mère. Bien au contraire, c'est précisément parce que l'embryon ne peut se nourrir et se développer qu'exclusivement aux dépens du plasma de sa mère qui, en plus de substances nutritives proprement dites, charrie toutes les substances autocoïdes et les harmozones de la mère et que le placenta n'empêche certainement pas de passer, que tous les changements de l'équilibre fonctionnel éprouvé par la mère auront une répercussion certaine sur l'enfant pendant la grossesse, après la naissance, pendant l'allaitement et, même encore, d'une façon indirecte, pendant l'éducation.

On peut même affirmer que c'est pendant la vie intra-utérine que le milieu agira le plus fortement sur l'hérédité, parce que son influence s'exercera non seu- 
lement sur les cellules somatiques du futur individu, mais, en même temps, sur les cellules germinales qu'il renferme, et qui, les unes et les autres, traversent à ce moment la période de développement la plus active, parce que le moindre changement provoqué dans les cellules embryonnaires, somatiques ou germinales, peut produire des effets relativement considérables dans l'équilibre de l'individu et de ses gamètes.

On constate aussi que les individus nés successivement des mêmes parents sont très souvent très différents les uns des autres, tant au point de vue de leur constitution que de leurs capacités et facultés psychiques. Ces différences peuvent avoir pour origine des chromosomes de provenances ancestrales différentes, dans les gamètes mâles et femelles qui ont formé les oospermes successifs; mais ces différences de structure et de caractère, ou l'apparition des caractères individuels nouveaux, ont aussi pour cause les changements de l'équilibre fonctionnel, que les parents ont subis aux différents moments de leur vie, coïncidant avec la maturation de leurs gamètes.

L'indication précise que l'on peut tirer de toutes ces données positives et de leurs interprétations, que nous venons de résumer, en ce qui concerne une intervention consciente et voulue dans les directions générales que l'on se proposerait de faire prendre aux modifications de l'hérédité, serait donc d'agir surtout sur la femme dès le début de la grossesse et pendant toute sa durée, ainsi que pendant toute la durée de l'enfance et de l'adolescence des enfants.

C'est certainement en agissant sur l'organisme de la femme, de façon à obtenir chez elle le plus parfait équilibre physique, intellectuel et moral, qu'on 
arriverait le plus rapidement à modifier l'hérédité de l'espèce humaine, dans le sens désiré : en augmentant la puissance de l'énergie physique en harmonie avec toutes les autres fonctions de l'organisme féminin, on agirait le plus sûrement et le plus rapidement sur le perfectionnement de l'espèce, parce que par ses fonctions de mère pendant la grossesse et d'éducatrice de ses enfants, ensuite, l'influence de la femme sur les modifications de l'hérédité est plus grande, plus immédiate et plus profonde que celle de l'homme.

Et, en agissant ainsi, l'homme ne sera plus un milieu de culture passif de son oosperme. En réglant d'une certaine façon, qu'il apprendra sans doute à connaître, la structure et les fonctions de ses glandes endocrines, il arrivera très probablement à reculer considérablement les limites de sa vie individuelle, augmentera par cela même, par un exercice approprié et par l'accumulation des expériences vitales, la puissance de son énergie psychique dans des proportions, qui dépasseront certainement tout ce que nous pourrions imaginer actuellement. Par l'hérédité convenablement dirigée, il stabilisera les caractères acquis, autant que cela sera nécessaire, et deviendra l'agent actif de cette évolution progressive de la matière vivante, dont il est encore aujourd'hui l'esclave à peu près inconscient.

\section{9. - Résumé et conclusions du livre III}

Tout ce que nous savons aujourd'hui des manifestations de l'évolution progressive de la matière vivante peut donc être résumé en quelques mots.

Depuis la formation des premières unités biolo- 
giques des êtres unicellulaires (nous n'avons pas de documents assez précis pour nous représenter exactement son évolution dans la période précellulaire), la matière vivante continue toujours à se développer et à s'accroître par la multiplication des organismes unicellulaires. Son évolution progressive continue consiste essentiellement dans la formation progressive pour cet organisme unicellulaire, d'un milieu d'évolution immédiat, de plus en plus différencié, doué, grâce à cette complexité croissante, d'une énergie propre et de plus en plus puissante et devenant par là à la fois plus résistante, plus stable et d'une sensibilité plus délicate à l'action des éléments toujours variants, du milieu extérieur.

Cette évolution s'est poursuivie ainsi par la formation alternative et la coordination des structures et des formes d'énergie nouvelles, étape par étape, d'une façon ininterrompue à travers des millions de siècles; d'une façon spontanée et inconsciente jusqu'à l'homme actuel, qui devient de plus en plus conscient du mécanisme de cette évolution, du rôle biologique de l'organisme unicellulaire impérissable et de son milieu immédiat, de l'individu périssable.

Et, grâce à cette énergie nouvelle, la conscience, résultant de la coordination des structures et des énergies préformées de plus en plus différenciées, l'homme cherche à intervenir en connaissance de causes et d'effets, dans le mécanisme futur de cette évolution progressive, à la dominer et à la conduire avec plus de sécurité et de certitude, dans la direction qu'elle semble devoir prendre d'elle-même, que la connaissance du passé lui indique comme la seule possible : l'accroissement illimité de l'énergie psy- 
chique et la différenciation illimitée de son organe, le pallium.

L'homme est arrivé ainsi à concevoir un but précis à ses efforts, un idéal d'avenir réalisable d'une évolution du corps et de l'esprit illimitée, parce que cette évolution porte en elle tous les éléments d'un accroissement structural et énergétique infiniment perfectibles. 
http://rcin.org.pl 


\section{CONGLUSIONS GÉNÉRALES}

\section{0. - La place de l'homme dans la nature}

Pour conclure, je voudrais donc faire remarquer avant tout, que le but de cet ouvrage n'était pas de faire un résumé, forcément incomplet, de l'ensemble des découvertes scientifiques. Ces découvertes sont exposées avec beaucoup plus de détails et de précision dans les ouvrages spéciaux, et mon but n'est autre que de choisir parmi ces découvertes celles, qui nous permettent de formuler les hypothèses les plus vraisemblables sur les règles les plus générales du mécanisme et de l'enchaînement des phénomènes qui se sont déroulés sur notre planète depuis son origine jusqu'à nos jours; de montrer comment cet enchaînement a abouti à la formation de l'homme et enfin quel est actuellement et quelle peut être dans l'avenir l'intervention de cette dernière unité "matière-énergie" dans l'évolution progressive.

Nous avons vu ainsi, que malgré son origine incontestablement commune avec toutes les autres unités organiques et inorganiques, tant en ce qui concerne son corps que son esprit, malgré qu'il ne peut être autre chose que le dernier produit, le plus parfait, de cette évolution progressive à partir des 
éthérons, l'homme, depuis qu'il est devenu conscient de sa pensée, a droit à une place spéciale dans la nature. Il devient le facteur conscient de cette évolution au lieu d'en être un instrument inconscient à l'égal de toutes les unités "matière-énergie " qui l'ont précédées.

Nous avons vu aussi que, par l'accroissement continu de la puissance énergétique des unités successivement formées, cette évolution doit être nécessairement illimitée, que l'objet actuel de cette évolution ne peut être autre que l'homme, et que, dans l'organisme humain, la différenciation, c'està-dire le perfectionnement, par l'accroissement de complexité et de puissance, portera sur le cerveau et sur son énergie propre: l'intelligence et la volonté.

Il est grand temps d'abandonner l'idée qu'il serait possible d'accroître la force morale de l'espèce en agissant simplement sur l'imagination de l'individu, en cherchant à lui inculquer un certain nombre de règles de conduite basées sur un idéal d'ordre purement sentimental.

L'histoire des guerres et des révolutions des temps anciens et de l'ère chrétienne, un coup d'œil jeté sur les conditions de la vie sociale actuelle, nous montrent jusqu'à l'évidence, que l'homme actuel est dominé, dans presque tous ses actes, bien plus par ses instincts que par son intelligence. Chacun de nous doit reconnaître que, dans la plupart des actes de sa vie, il est bien plus entraîné par ses passions que par sa raison! Et que c'est ainsi, malgré les trente ou quarante siècles d'enseignement moral religieux.

N'est-il pas évident que cet enseignement n'a pas donné les fruits qu'il prétendait ensemencer? Et 
ne faut-il pas reconnaître qu'il ne pouvait pas en être autrement, que des commandements des apôtres, l'humanité ne pouvait retenir et s'assimiler que les gestes, tandis que l'idée devait nécessairement rester lettre morte, parce qu'elle ne s'adaptait pas à l'équilibre cérébral et psychique de l'homme d'alors et ne s'adapte pas encore à cet équilibre de la grande majorité des hommes aujourd'hui.

Nos sentiments sont des manifestations de nos instincts et non de notre raison. Leur niveau plus ou moins élevé est fonction, est le produit, du degré de développement de la conscience et de la compréhension de cette conscience, c'est-à-dire de l'intelligence et de la volonté.

Pour que les manifestations de justice, de solidarité sociale et de bonté deviennent des besoins spontanés et se manifestent par des actes toujours et partout, il faut qu'elles puissent procéder d'un équilibre psychique profond et d'une compréhension de cet équilibre, c'est-à-dire d'une plus haute intelligence des choses et non pas d'une croyance, d'un commandement imposé par un idéal de justice et de bonté abstraites.

Les cerveaux, dont les centres d'association sont assez développés et différenciés pour comprendre ces choses, sont certainement très nombreux aujourd'hui; on voit ces préoccupations percer dans les écrits de nombreux biologistes, sociologues et ingénieurs, surtout dans les pays neufs, moins gênés par les éléments de structure, les traditions (par exemple aux Etats-Unis), mais trop peu nombreux sont encore ceux qui pourraient, qui voudraient, qui oseraient rompre franchement avec le passé et chercher à réaliser ces préoccupations par des actes.

Dsxysz. - La genèse de l'énergie psychique. 


\section{1. - Orientation actuelle de l'évolution}

Autant qu'il est possible de comprendre l'orientation de l'évolution actuelle, on peut y distinguer, comme c'est le cas d'ailleurs pour bien d'autres phénomènes, deux tendances antagonistes: d'une part, à l'uniformisation ou nivellation de tous les individus de l'espèce "homme»; d'autre part, à la différenciation des races, des nations, des classes sociales, des familles. Et on peut dire, que ces deux tendances ont leurs racines profondes dans les lois générales, qui règlent le mécanisme de l'évolution progressive.

On constate, en effet, qu'à mesure que l'individualisation des organismes, qui se sont succédés depuis les protistes, devient plus forte, plus nettement accusée, le nombre des espèces dominantes diminue. Ainsi le nombre des espèces d'êtres unicellulaires, des végétaux et des invertébrés est, pour ainsi dire, incalculable et continue à s'accroître. On en découvre tous les jours de nouvelles et nous avons vu que, dans la classe des microbes, il se forme ou on peut former des espèces nouvelles.

On peut en dire autant des invertébrés inférieurs, des poissons, dont le nombre d'espèces est plus grand aujourd'hui qu'à l'époque primaire et secondaire.

A partir des batraciens, on voit, au contraire, que le nombre des espèces a passé par des "maxima" pour diminuer ensuite. Le maximum de développement des batraciens coïncide avec le début de l'époque secondaire, celui des reptiles avec la fin de cette période, celui des mammifères, excepté l'homme, avee le milieu de l'époque tertiaire.

En ce qui concerne l'homme, les documents paléontologiques tendent à prouver, qu'à la fin ter- 
tiaire et au début du quaternaire, les hominiens étaient représentés par plusieurs genres, comprenant plusieurs espèces : l'anthropopithèque de Java, l'homme de Heidelberg, de Neanderthal, de Dawson, qui coexistaient avec les ancêtres directs de l'homme actuel et dont une seule espèce, l'homo sapiens ou faber (Bergson) a évolué jusqu'aujourd'hui.

L'évolution spontanée d'une espèce, réglée par les seules lois de la nature inconsciente; peut donc être représentée par un fuseau. Elle commence par une famille, dont les descendants se différencient en se multipliant et en vivant dans des conditions de milieu différentes et peuvent devenir ainsi des souches de races et d'espèces différentes, pendant que chez ceux des descendants de cette famille, qui ont continué à vivre dans les mêmes conditions de milieu, les caractères de la race primitive s'affermissaient par une hérédité de plus en plus longue. Alors deux éventualités peuvent se produire : ou bien ce seront les descendants directs de la première famille qui, ayant trouvé les meilleures conditions de développement, résisteront le plus longtemps et formeront une espèce représentée par un très grand nombre d'individus de familles et de races de la même espèce, ou bien c'est une ou plusieurs branches collatérales, issues des différents niveaux du fuseau primitif, qui se développeront mieux ou aussi bien, que les descendants directs, et il y aura simultanément un faisceau de fuseaux ou un genre composé de plusieurs espèces, issues de la même souche. 


\section{2. - Classification des hommes suivant le degré de développement de leur encéphale et de leur éner- gie psychique.}

$\mathrm{Au}$ point de vue zoologique, l'humanité actuelle forme dans son ensemble une seule bonne espèce. En se plaçant à un point de vue biologique plus général, on devrait joindre à l'espèce "homme ", l'espèce voisine des singes antropomorphes, parce que les propriétés et par conséquent la composition chimique de leur sang est la même que celle de l'homme proprement dit; mais si on envisage ces choses au point de vue de la différenciation des centres d'association et de la puissance de l'énergie psychique, on pourrait déjà diviser les individus " hommes zoologiques" en plusieurs espèces. Ainsi, pour les biologistes, le genre des hominiens se trouverait près de l'extrémité descendante du fuseau de son évolution; pour les zoologistes, elle se trouverait exactement au point de jonction de deux fuseaux successifs; pour le sociologue, elle aurait déjà dépassé ce point et serait actuellement au début de la branche ascendante d'un fuseau nouveau, dont l'origine serait peut-être contemporaine de l'âge du renne.

Les documents de l'industrie humaine de cette époque semblent prouver, en effet, qu'au point de vue de leur développement mental, les races des hommes de ces temps accusaient des différences moins grandes que celles que nous constatons entre les individus des races humaines d'aujourd'hui, par exemple entre les Australiens, les negroïdes et les savants des peuples civilisés. On peut admettre aussi que la nouvelle différenciation a débuté au commen- 
cement de l'âge du cuivre avec le peuple qui, le premier, a commencé à travailler les métaux et qu'elle a continué à s'accentuer jusqu'à ces derniers temps, jusqu'au moment où les facilités des moyens de communication ont permis à l'homme blanc de pénétrer partout et d'intervenir activement dans le développement des autres races.

Jusqu'à présent, ce ne sont généralement pas les savants des peuples civilisés, qui se sont occupés du "bonheur " de leurs frères des races inférieures et l'intervention de ceux qui s'en sont arrogés le droit a eu pour résultat la disparition complète de certaines tribus et même de certaines peuplades: des Tasmaniens, des Hereros dans l'Afrique du Sud, de certaines tribus indiennes de l'Amérique du Nord.

Quels seront les effets de l'intervention consciente de l'homme dans la différenciation et le développement non seulement des races inférieures, mais de toute l'humanité ?

Ce qui semble certain, c'est que la classification des hommes ne pourra plus être basée exclusivement sur les caractères de leurs éléments de structure, Ainsi, les mesures des capacités des crânes ont donné : 1.340 centimètres cubes pour la moyenne des Australiens, 1.550 pour les Parisiens et 1.646 pour les Esquimaux, et on a constaté (Marcelin Boule) qu'il y a très peu de différence entre la capacité crânienne de l'homme fossile de la Chapelle-aux-Saints (débuts du quaternaire) et celui du savant paléontologiste américain Cope.

La classification des hommes actuels et surtout celle des hommes futurs devra reposer principalement sur les détails histologiques de la structure et de la composition chimique des cellules qui forment 
l'encéphale et sur le degré de puissance de son énergie psychique, ce qui ne doit d'ailleurs pas empêcher les naturalistes de rechercher les similitudes et les différences anatomiques et physiologiques et d'établir, d'après ces caractères, des classifications d'autres natures.

\section{3. - Le rôle de la pédagogie dans l'éducation et dans l'enseignement}

Nous avons vu, dans le chapitre consacré à l'évoIution du tissu nerveux, que le développement des diverses formes de l'énergie psychique et de l'apparition des formes ou qualités nouvelles de cette énergie était fonction du développement du pallium et, dans ce pallium, des centres spéciaux dans les sphères sensorielles et les sphères d'association, ou plutôt que l'élévation graduelle de l'ensemble des facultés mentales résultait de l'enchaînement des réactions successives réciproques entre les éléments anatomiques et histologiques et les facultés mentales : un centre nouveau fait apparaître une faculté nouvelle ou d'un degré de développement plus haut et cette forme d'énergie nouvelle provoque à son tour la formation de centres nouveaux ou plus différenciés.

Nous avons cherché à démontrer que ce développement progressif et simultané, d'une part, du volume et de la différenciation du pallium, d'autre part, de la puissance de l'ensemble des énergies psychiques, a eu et aura pour facteur principal la recherche et la connaissance de plus en plus précise du mécanisme de plus en plus intime des phénomènes, qui concourent à l'évolution du milieu exté- 
rieur dans lequel nous vivons et du milieu intérieur, de notre individu vivant. Le rôle de la biologie, y compris la psychologie expérimentale, sera de déterminer avec une précision et une exactitude de plus en plus grandes, les relations d'organes à fonctions de différents centres et des différentes facultés psychiques plus ou moins développées chez différents individus.

Mais ce serait sortir des cadres de ce livre, que d'entrer ici dans les détails de la marche à suivre pour continuer à gravir les échelons de l'évolution ascendante plus rapidement et avec moins d'efforts pénibles, que cela n'a pu se faire jusqu'à présent.

Ce sera la tâche de la pédagogie qui, avec le temps, règlera de mieux en mieux, d'une façon plus conforme aux besoins réels du corps et de l'esprit, l'instruction et surtout l'éducation de l'enfance et de l'adolescence, ainsi que la conduite dans la vie, de l'homme et de la femme adultes.

On dirait, qu'actuellement encore, le but de tout enseignement, dans presque toutes les écoles des pays civilisés, n'est pas de développer les facultés dominantes des élèves, de leur apprendre à travailler, mais de leur faire passer un examen ou un concours en vue d'une carrière, et il est évident qu'on donne ainsi la prime à la mémoire, au lieu de chercher à développer les qualités de l'intelligence, de l'imagination et de l'initiative.

Mais déjà de nombreuses voix s'élèvent contre ces méthodes qui, à tout-homme qui réfléchit, semblent de véritables anachronismes. Terman et Goddard, aux Etats-Unis, Burth, en Angleterre, Stern, en Allemagne, Binet, Jules Payot, en France, cherchent à modifier cet état de choses. 
Pour choisir les hommes les plus aptes à devenir de bons officiers pour l'armée en formation pendant la dernière guerre, le gouvernement des Etats-Unis a institué un army mental test ou l'épreuve par laquelle l'examinateur cherchait surtout à juger, non pas la somme des connaissances du candidat, mais sa capacité d'utiliser celles qu'il possède. A Louisville, on sélectionne ainsi les élèves les plus capables et on a créé pour eux des classes spéciales de "surnormaux ", où ils peuvent progresser deux fois plus vite que les autres. En Angleterre, en Allemagne, on cherche, par des épreuves psychologiques, à faire un choix entre les élèves possédant des aptitudes naturelles différentes. En France, J. Payot, dans une série d'ouvrages (1) qu'on ne saurait trop recommander à tous ceux qui se destinent à l'enseignement ou qui le font déjà, insiste surtout sur l'organisation du travail intellectuel et indique les méthodes par lesquelles on peut rendre ce travail moins pénible et plus profitable, aussi bien aux élèves qu'aux professeurs, comme l'a fait le système Taylor pour le travail manuel des ouvriers.

Dans toutes ces tentatives, on se préoccupe tout autant du développement du corps que de l'esprit. Dans toutes, on voit la préoccupation d'un équilibre fonctionnel de tous les organes, équilibre qui, s'il n'est pas toujours nécessaire au développement de certaines facultés de l'individu (2), est indispensable au développement progressif de l'espèce.

(1) L'éducation de la volonté. Le travail intellectuel et la volonté. Félix Alcan, Paris.

(2) On ne connait pas de lignées de plusieurs générations d'hommes d'une intelligence ou d'un talent très supérieurs, parce que leur supériorité, due, le plus souvent à une hyper- 
Ces méthodes, quand leur application sera généralisée et appliquée non seulement aux enfants et aux adolescents, mais aussi plus tard aux hommes et aux femmes adultes, au moment du choix des candidats pour des emplois quelconques, auront encore l'avantage inappréciable de nous montrer quels sont les moments de développement de toutes les formes d'énergie par rapport à l'âge chez les différents individus, en quoi consistent ces différences et enfin quelles peuvent en être les causes.

\section{4. - Tendance à une différenciation et à une sélec- tion des individus d'une mentalité de plus en plus élevée.}

Il est impossible de ne pas reconnaître dans tous ces efforts une tendance à une différenciation et à une sélection des individus les plus actifs, les mieux doués, et il n'est pas douteux que si on continue à favoriser le développement de certaines aptitudes et dons naturels, cette différenciation ne peut que s'accentuer avec le temps, malgré l'action antagoniste des idées de liberté et d'égālité mal comprises.

Un être unicellulaire, une amibe, un globule blanc du sang, réagit différemment aux excitants de différentes natures. Il n'englobera pas indifféremment tous les corps qu'il trouvera sur son chemin ou qui s'en approcheront; il éprouvera une attraction pour les uns, une répulsion pour d'autres, il choisit, mais il n'est pas libre de repousser ce que son chimiotac-

trophie de certains organes, n'est pas héréditaire. On trouve par contre des exemples de familles dans lesquelles une forte intelligence moyenne se maintient ou même progresse lentement pendant plusieurs générations successives. 
tisme l'oblige à englober et à digérer, ni d'attirer ce que son tactisme repousse. Ses réactions, dues à une sensibilité générale non différenciée, sont toutes obligatoires, parce qu'elles sont purement chimiques et, pour ainsi dire immédiates. Et si un leucocyte attire et digère plus rapidement et plus facilement un microbe qu'il a déjà digéré une ou plusieurs fois, ce ne sera pas là un effet de mémoire ou d'expérience, mais simplement d'une modification de son équilibre physico-chimique. La quantqité des substances de son milieu intérieur, qui l'attirent vers le même microbe et digèrent ce dernier, aura augmenté à chaque répétition de la même opération.

Les pucerons du rosier, placés dans un tube de verre, à la lumière diffuse, et garni de feuilles de toutes sortes et de feuilles de rosier, finiront par se placer tous sur ces dernières; mais si on recouvre le tube de papier noir, de façon à intercepter la lumière partout, excepté à son extrémité fermée, et si on tourne cette extrémité vers la lumière, tous les pucerons, sans exception, quitteront les feuilles pour se coller aux parois de verre de l'extrémité du tube éclairé, où ils périront d'inanition (1). Ils ont donc la faculté de choisir parmi les feuilles de plusieurs espèces, et parmi celles du rosier, les plus fraîches ou les plus jeunes; mais ils ne peuvent pas désobéir à l'appel de la lumière et n'apprennent pas par l'expérience qu'ils se condamnent ainsi à la mort.

Les pucerons sont donc libres de choisir parmi les excitants de certaines catégories et doivent obéir passivement à d'autres.

Chez les organismes plus élevés, le mécanisme

(1) Expérience de Jacques LoEB: Conception mécanique de la vie. Félix Alean, Paris. 
des réactions aux excitants du milieu extérieur devient plus complexe. Il n'y aura plus d'obéissance passive et immédiate, parce que toute action d'un excitant extérieur sera contrôlé par une série de réactions du milieu intérieur, pourvu d'une mémoire et de connaissances acquises par l'expérience, et l'acte par lequel l'organisme répondra définitivement à l'excitation, sera la résultante d'un choix entre les possibilités plus ou moins nombreuses, suivant son degré de différenciation, de la variété et de la précision de ses connaissances.

C'est à cette faculté de choisir entre les possibilités de réagir d'une façon ou d'une autre, qui existe chez tous les animaux pourvus d'un système nerveux différencié et que l'homme possède à un degré d'autant plus haut qu'il est plus instruit, que l'on a donné le nom de liberté. C'est sur cette conception de liberté que l'on a établi les responsabilités individuelles et sociales, les méthodes d'éducation et de justicé.

Et s'il en est ainsi, peut-il être question d'une liberté absolue, à un degré quelconque, chez un être vivant et chez l'homme, de ce qu'en philosophie on appelle: le libre arbitre?

Certainement non !

Non seulement l'homme est incapable et ne sera jamais capable d'exécuter et même de concevoir un acte arbitraire quelconque, mais l'homme le plus instruit est limité dans le choix des possibilités de ses conceptions et de ses actes par son hérédité d'abord, par son éducation, son instruction, le milieu immédiat, la société, la nation dans laquelle il évolue. Et on peut même dire que si l'homme riche, qui dispose de moyens d'action puissants, a plus de liberté de 
choisir entre les conditions d'existence qu'il croit lui convenir, l'homme instruit sera d'autant moins libre d'agir à sa guise, que ses connaissances lui auront tracé des lignes de conduite plus fortement accusées et plus précises.

Il saura de mieux en mieux maîtriser et coordonner ses intincts, mais obéira d'une façon de plus en plus irrésistible à sa raison. L'humanité n'est pas plus libre de désobéir aux lois inéluctables de l'évolution que les protozoaires de résister aux excitants du milieu extérieur.

L'homme a l'illusion d'être libre parce que, en se décidant d'agir d'une certaine façon, il sait qu'il aurait pu agir de toutes sortes d'autres façons, mais pourra-t-il choisir librement le ou les mobiles qui fixeront son choix définitif quel qu'il soit? Il est tout autant obligé d'aller à la lumière, au propre et au figuré, que le puceron de l'expérience de Loeb.

Seulement, à mesure que les organismes évoluent, que leur milieu intérieur devient plus complexe, sa sensibilité plus différenciée et plus délicate, les excitants du milieu extérieur passent par des réflecteurs de plus en plus nombreux, délicats et précis, avant de produire un effet, qui sera la résultante de toutes ces réactions successivement réfléchies par certains centres sensibles et coordonnées par d'autres.

Le milieu intérieur gardera une mémoire d'autant plus durable et plus précise des réactions qu'il aura subies de la part du milieu extérieur, qu'il sera plus différencié et par là plus fortement individualisé. Cette mémoire provoquera l'apparition des mobiles des réactions spontanées des "révélations" intérieures, sans intervention d'aucune excitation extérieure immédiate, et ce sont ces révélations involon- 
taires qui deviennent les mobiles de nos actions volontaires.

Nous n'avons pas le pouvoir de faire apparaitre ces mobiles à volonté, pas plus que nous ne pouvons empêcher l'apparition de ceux qui se manifestent spontanément dans notre conscience; mais nous savons que ces mobiles ont leur origine dans le savoir acquis par l'expérience et cette expérience nous apprend en outre quels sont les actes en harmonie avec les lois du " jeu " de notre milieu familial, social, ou national. Et c'est ainsi que peut être justifiée la responsabilité de l'individu devant sa propre conscience et devant la justice du milieu, dans lequel il vit, justifiée, bien entendu, dans la mesure dans laquelle ce milieu a pris soin d'éveiller la conscience, la raison et la volonté de l'individu par une éducation appropriée. Par son jeu de réactions réciproques 'du milieu à l'individu, et de l'individu au milieu, la conscience de cette responsabilité, devient de plus en plus puissante et par là l'un des facteurs dominants de l'évolution ascendante.

\section{5. - Classification des hommes suivant la nature des mobiles qui les dirigent dans leurs actions}

Une dernière question se pose à ce propos :

Quelle sera la direction générale de l'éducation que la société tendra à donner à ses individus, ou, en d'autres termes, de quelle nature seront les mobiles qui s'éveilleront dans le milieu intérieur, dans la conscience des hommes du plus prochain avenir, par la force même des lois de l'évolution?

La façon la plus simple de trouver à cette question 
la réponse la plus satisfaisante, sera encore celle, que nous avons adoptée pour répondre à toutes celles qui se sont présentées dans le cours de cet exposé : par l'analyse du passé, de l'évolution de ce phénomène à partir de ses manifestations les plus simples.

On peut dire que l'amibe obéit dans ses mouvements et dans ses réactions internes, exclusivement à des excitations purement chimiques. Les seuls mobiles de ses actes procèdent des propriétés chimiques de ses micelles et des éléments qui composent ces micelles. Ces possibilités de choix sont réglées par les modifications, que l'équilibre physico-chimique de l'ensemble de la cellule peut supporter, sans que la cellule soit détruite. Il en est de même chez les plantes, qui obéissent, elles aussi, exclusivement et impérieusement à des tropismes (geo, helio, chimio, hydrotropismes).

Les animaux inférieurs s'affranchissent de certains tropismes mais obéissent aussi impérieusement aux seuls mobiles qui procèdent de la sensation de la faim et de la soif. Leurs possibilités de choix se bornent à la préférence des aliments qui conviennent le mieux à leur équilibre physico-chimique et qui se manifeste par le goût et par l'odorat, aidés par les autres sens, s'il y en a.

L'expérience leur apprend à connaître les exigences de cet équilibre physico-chimique. Chez les vertébrés, les tropismes ne provoquent plus que des réactions indirectes. Les poissons, les batraciens, les oiseaux seront encore attirés par la lumière, mais ne se brûleront plus à la flamme, comme les papillons. Les mobiles, qui.les feront agir, auront leurs origines encore principalement dans la sensation de la faim qui devient l'instinct de conservation individuelle, 
mais à cet instinct égoïste s'ajoute peu à peu l'instinct altruiste de la conservation de l'espèce, déterminé par les affinités des cellules sexuelles et chez les vertébrés supérieurs apparaîtront les sentiments désintéressés d'amitié et d'amour. Certains animaux domestiques, les chiens en particulier, sont capables d'actes de dévouement contraires à leur instinct de conservation, dictés par des sentiments qui deviennent quelquefois des mobiles des réactions plus impérieuses que les instincts égoïstes.

Enfin, chez l'homme, apparaît un mobile nouveau, la raison qui, à mesure qu'elle dispose des moyens d'action plus étendus, d'une conscience des choses plus vaste et plus éclairée, domine de plus en plus toutes les catégories de mobiles précédemment formés et se manifeste par des actes d'une volonté de plus en plus ferme et précise. Ainsi, tout en étant soumis aux obligations irrésistibles d'une hérédité imposée par les propriétés des éléments, dont il est formé et dont l'origine remonte aux éthérons, l'homme a été amené, par la force même des lois de son évolution, à diriger cette évolution par la raison, et l'humanité n'est pas plus en état de s'opposer à cette loi, qu'une amibe n'est capable de résister aux affinités de son équilibre chimique.

L'homme n'est pas libre de choisir les mobiles de ses actions; mais il a conscience de ses connaissances et de son expérience, qui lui confèrent des possibilités d'autant plus nombreuses dans le choix de ses réactions, que son savoir est plus vaste.

Il est responsable de ses actes parce qu'il peut apprendre par l'expérience, quels sont les actes en harmonie avec les conditions d'existence du milieu dans lequel il vit. 
L'humanité n'est pas et ne sera jamais libre d'évoluer dans une direction arbitrairement choisie.

L'évolution progressive qui subissait jusqu'à présent, successivement, les lois des réactions tropiques, réflexes, instinctives et sentimentales subira désormais, de plus en plus impérieusement, les lois de la raison, qui est elle-même une résultante du concours coordonné de toutes les facultés de l'énergie psychique, et qui se sera manifestée dans les cerveaux des dirigeants du moment, sélectionnés par une différenciation de plus en plus grande et délicate des individus.

Depuis les temps historiques, l'évolution progressive de l'humanité tend à une différenciation de plus en plus grande des individus, mais l'unité de mesure de cette différenciation, l'appréciation de la valeur des caractères qui justifieront le classement des individus en espèces ou classes sociales différentes, ne seront pas les mêmes pour chaque époque de l'évolution.

On peut dire que depuis le début des temps historiques jusqu'à une époque que l'on peut délimiter par la grande révolution française, la classification sociale des hommes reposait presque exclusivement, en fait sinon en idée, sur des besoins d'ordre physique, sur des privilèges accordés à la puissance des moyens d'action matériels des individus, des familles, des races et des peuples, sur une conception égoïste (individuelle, familiale ou nationale) de la propriété des biens matériels fournis par la nature ou par l'industrie. Les hommes étaient classés suivant la quantité de ces biens qu'ils détenaient, et les individus, qui ne possédaient pas assez de ces biens accumulés pour subvenir aux besoins de leur exis- 
tence, étaient obligés d'obéir aux ordres de ceux qui pouvaient leur fournir ce supplément de biens en échange de l'exécution des ordres reçus. Les possibilités des réactions spontanées des salariés, leur liberté de choisir parmi ces possibilités, étaient donc limitées par l'intervention des mobiles étrangers à leur milieu intérieur.

L'état d'esprit qui a justifié un tel état de choses dans le passé par les diflérences d'instruction et d'éducation entre les dirigeants et les dirigés et par la nature du savoir des dirigeants, domine encore aujourd'hui les relations entre les hommes de la même race et entre les peuples de races difiérentes; mais n'est-il pas évident que, depuis que la raison a commencé à puiser les mobiles de ses actes dans la science expérimentale, les jours de cette domination sont comptés?

La différenciation sociale continuera à suivre son cours. Les différences entre les individus différemment doués deviendront de plus en plus accusées; mais leur répartition en classes ne sera plus basée sur la valeur des moyens dont ils disposeront pour assurer leur existence. Elle sera basée uniquement sur le degré d'harmonie et de puissance de leur énergie psychique, naturelle et acquise.

La quantité totale de richesses naturelles de la terre suffit et suffira toujours aux besoins de subsistance des hommes, et si aujourd'hui encore l'immense majorité des individus sont obligés d'aliéner une fraction plus ou moins grande de leur liberté pour vivre, c'est uniquement, parce que les conditions de la production ainsi que la répartition des richesses ne sont pas organisées, comme la raison indique qu'elles. pourraient l'être.

Danysz. - La genèse de l'énergie psychique. 
Cet état de choses était une nécessité de l'évolution d'une époque, qui s'appellera probablement dans l'histoire de l'humanité l'époque des sentiments et des passions insuffisamment contrólés par la raison. Ce n'était ni déraisonnable, ni injuste parce que, pour la majorité des dirigeants et des dirigés, cet état de choses résultait de leur mentalité, de leur idéal du devoir, du droit et de la justice; mais il est certain que ce serait très déraisonnable et injuste de persister à vouloir maintenir cet état de choses du moment, où, par la force de l'évolution, un idéal du devoir, du droit et de la justice basé sur une conception mieux raisonnée des relations sociales et du mécanisme de l'évolution, s'est fait jour dans la conscience d'un nombre suffisamment grand d'individus.

L'évolution progressive ne s'arrêtera pas dans son mouvement ascensionnel malgré tous les obstacles qu'elle pourra rencontrer sur son chemin, parce que rien ne peut s'opposer à l'entrée en action d'une énergie nouvelle, quand cette énergie résulte de la coopération des unités matière-énergie précédemment formées.

Une fois commencé, ce règne de la raison s'affirmera avec autant de force irrésistible par une différenciation de plus en plus délicate des sphères d'association, que s'est affirmé le règne de l'affinité chimique par la formation des atomes, des tropismes, des réflexes, des réactions instinctives et sentimentales, par la formation du plasma vivant, et ensuite dans ce plasma des différenciations successives des organes des sens et des centres nerveux et cérébraux.

Mais cette évolution peut être plus ou moins accélérée ou retardée, suivant que les facteurs agissants seront plus ou moins en harmonie avec l'équilibre 
social de l'organisme (social), sur lequel ils auront à agir et surtout suivant l'énergie avec laquelle ils agiront.

A l'égal d'un réactif qui peut provoquer dans un être vivant des réactions stimulantes ou pathogènes, suivant sa nature et sa dose et suivant le nombre, la nature et le rôle des cellules atteintes, une idée ou une méthode mise en action dans une société, contribuera au progrès ou l'arrêtera pendant un temps plus ou moins long, suivant la façon dont elle sera appliquée.

Les idées et les recherches de Lamarck, Darwin, Weissmann, Pasteur, J. Loeb, etc., sont stimulantes et évolutives, paree qu'elles enrichissent le savoir, éveillent le désir de recherches nouvelles plus précises et ne conduisent à des idées de réformes sociales que progressivement, par un long travail de différenciations nouvelles des cellules cérébrales en harmonie avec les fonctions du reste de l'organisme et par la formation d'une mentalité nouvelle, qui en est la conséquence.

Les idées d'un Marx, peu constructives, parce qu'elles ne reposent que sur des spéculations mentales accessibles à un petit nombre d'esprits, deviennent destructives parce qu'elles peuvent être mises en action immédiate à la faveur des circonstances fortuites et éveillent alors, non par les désirs de recherches nouvelles plus fécondes, mais des passions, dont les manifestations deviennent d'autant plus contraires au progrès, qu'elles se déchaînent dans des consciences plus obscures. 


\section{6. - L'évolution ne connaît pas de révolutions}

La transformation d'une cellule épidermique ou épithéliale en cellule nerveuse se fait sans aucun cataclysme violent et si les révolutions politiques sont généralement sans danger pour l'évolution progressive, parce qu'elles n'influent pas beaucoup sur les travaux des savants, les révolutions économiques, même des réformes justes, mais trop brusques inspirées par des considérations politiques qui n'ont rien à voir avec le progrès, amèneront toujours des perturbations profondes et contraires au progrès.

La loi de huit heures appliquée en France au moment où le nombre des travailleurs a diminué d'un dixième, a abaissé la production générale, agricole et industrielle d'un quart et il en est résulté un tel renchérissement de tous les objets de première nécessité, que les travaux désintéressés de tous les laboratoires scientifiques en ont été presque complètement arrêtés.

Une réforme de cette nature ne devient possible et réellement profitable, que si la diminution du travail manuel est préalablement compensée par un perfectionnement de l'outillage, qui rendra la production proportionnellement plus rapide.

\section{7. - Le chemin du bonheur plus grand pour le plus grand nombre}

Mais malgré ces bouleversements, qui heureusement, ne seront probablement jamais universels et simultanés, l'évolution ne s'arrêtera pas.

A mesure qu'il s'affirmera par ses effets, le règne de la raison et de sa résultante, la volonté, s'affer- 
mira de plus en plus. Les sciences physiques chercheront à remplacer de plus en plus la main-d'œuvre pénible par le travail des machines, c'est-à-dire, en dernière analyse, le travail des muscles par̃ le travail du cerveau, infiniment plus productif; les sciences biologiques cherchent et ne cesseront de chercher à régler de mieux en mieux les conditions de nutrition et de différenciation des tissus, pour arriver à un développement progressivement plus harmonieux de tous les organes; les sciences pédagogiques et sociales, à favoriser l'exercice et le développement de la conscience, de l'imagination, de l'intelligence et de la volonté, en diminuant pour tous la durée des travaux obligatoires, à favoriser le développement des dons individuels de chacun et à appeler ainsi à la sélection des dirigeants les individus le mieux doués, de plus en plus nombreux.

On constate toutes ces tendances aujourd'hui dans tous les esprits éclairés, et il ne peut pas en être autrement, parce que, pour la raison, la tendance à une harmonie universelle dans les rapports sociaux, $\grave{a}$ une justice respectant les droits de chacun à disposer librement de ses possibilités personnelles, est un besoin aussi impérieux, qu'une réaction tropique pour un amibe.

Quels seraient les moyens les plus sûrs, le chemin le plus court, pour arriver à ce bonheur plus grand pour le plus grand nombre?

On peut affirmer avec certitude qu'on ne trouvera ces moyens que dans le développement des sciences expérimentales, surtout des sciences biologiques, y compris les sciences sociales. Et quand on fait le bilan, siècle par siècle d'abord, décade par décade ensuite, des progrès accomplis dans le domaine de 
ces sciences, on constate, que le développement des facultés cérébrales suit une marche ascendante de plus en plus rapide et que le nombre d'individus qui participent à ce progrès augmente dans la même proportion. On peut donc en déduire que le moment où l'homme saura mettre en valeur, à son profit, l'énergie emmagasinée dans les atomes qui composent notre planète et peut-être aussi dans ceux de l'espace, et diriger d'une façon consciente la nutrition ainsi que la différenciation de ses tissus, le moment où il deviendra conscient du mécanisme intime des réactions de son milieu intérieur et extérieur n'est pas très éloigné.

Ce ne sera peut-être pas plus que dans quelques dizaines de siècles, peut-être plus tôt, si toutefois un cataclysme cosmique ou social ne vient pas troubler pour trop longtemps l'équilibre normal de cette évolution, en bouleversant les lois de la sélection naturelle.

Mais, si l'évolution progressive n'est pas une illusion, si c'est une réalité, comme tous les documents connus aujourd'hui le prouvent, il viendra un moment où l'énergie psychique de l'homme, son savoir, sa raison et sa volonté toute puissante égalera celle dont l'imagination des prophètes a doté l'être suprême.

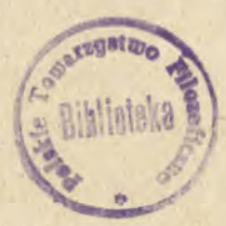




\section{TABLE DES NOMS DES AUTEURS CITÉS}

\begin{tabular}{|c|c|c|c|}
\hline & & & \\
\hline & 192 & De la Paz (D.) & 180 \\
\hline drow & 23 & Dejerine.... & 70 \\
\hline ataillon & 136 & Delage & 137 \\
\hline elfanti et Carbone. & 155 & Devaux & \\
\hline aude Bernard .... & 168 & e Vries & \\
\hline Berzélius ......... & 39 & ewitz & 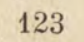 \\
\hline inet & 279 & er & 17 \\
\hline ohn (G.).. 122,123 & 180 & $\mathrm{ch}$ & 2 \\
\hline Bohr ................ & 9 & Duclaux (J.). & \\
\hline Bordet (J.). . & 155 & Dumas & 18 \\
\hline Boule (M.) ...... 84 , & 277 & Drze & 18 \\
\hline $30 \mathrm{v}$ & 264 & $\mathrm{~g}$ & 20 \\
\hline et Morris.. & 23 & uez & 21 \\
\hline Séquard ...... & 168 & $\mathrm{nn}$ & 19 \\
\hline$y \ldots \ldots \ldots$ & 23 & g. $67,70,71,72$ & 2,7 \\
\hline$\ldots \ldots \ldots$ & 279 & Friedberger ..... & 16 \\
\hline 218 & 229 & Funk (Casimir). 194, & 19 \\
\hline 1 et Wood.. & 177 & tov & 25 \\
\hline J.-B.) ....... & 180 & & 27 \\
\hline ne ........ & 176 & vski . & 13 \\
\hline & 155 & & 19 \\
\hline & 182 & & \\
\hline Clarke ........ 122, & 204 & Gudernatch .... & 12 \\
\hline $\begin{array}{l}\text { Conklin (D.-P.). } 122, \\
260,263 .\end{array}$ & 124 & Haeckel ........... & \\
\hline Crookes & 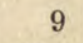 & Hay & \\
\hline Darwin & 01 & Herbst & \\
\hline
\end{tabular}




\begin{tabular}{|c|c|c|c|}
\hline & $P$ & & Pages \\
\hline ertwig & 204 & Payot (J.). & 280 \\
\hline er .......... & 70 & Pitz .... & 195 \\
\hline ins et Wilcock.. & 192 & incaré (Henri)... & 94 \\
\hline ns $\ldots \ldots \ldots \ldots$. & 123 & (Cajal).... & 170 \\
\hline $11 \ldots \ldots \ldots \ldots$ & 180 & et (Charles) .... & 163 \\
\hline alewsky ....... & 65 & V.) $\ldots \ldots \ldots$ & 121 \\
\hline$s \ldots \ldots \ldots$ & 155 & ford .... & 年 \\
\hline rck ..... 120, & 291 & abanejew ........ & 23 \\
\hline in $\ldots \ldots \ldots$. & 17 & s $\ldots \ldots \ldots \ldots$ & 70 \\
\hline s) $\ldots$ & 83 & er . & 256 \\
\hline$\ldots \ldots$ & 111 & $\ldots$ & 22 \\
\hline$\ldots \ldots$ & 23 & onds & 195 \\
\hline (2) & 136 & . $\quad 10$, & 109 \\
\hline e (Olivier)...... & 93 & er (Herbert)... & 200 \\
\hline eb .. $136,180,250$ & 291 & St & 193 \\
\hline$\ldots \ldots \ldots$ & 17 & . & 279 \\
\hline A.) $\ldots \ldots \ldots$ & 19 & $\ldots$ & 264 \\
\hline $\operatorname{lum}_{1} \ldots \ldots \ldots$ & 195 & . 122 , & 204 \\
\hline & 17 & $\ldots \ldots \ldots \ldots$ & 85 \\
\hline .) $\ldots \ldots$ & 123 & $n \ldots \ldots \ldots \ldots$ & 279 \\
\hline co ......... & 17 & a (J.-J.) ..... & 9 \\
\hline ... 217,218, & 2 & Broeck .... & 9 \\
\hline$\ldots \ldots \ldots \ldots$ & 191 & uchten. 67,74 , & 70 \\
\hline aikoff. 176,218 , & 229 & n... 121, 204, & 217 \\
\hline nikoff. $\quad 156,176$ & 239 & 1. & \\
\hline & & & 204 \\
\hline$\ldots \ldots \ldots \quad 192$ & , 19 & V & 192 \\
\hline ow $\ldots \ldots \ldots$ & 70 & V & 264 \\
\hline te $\ldots \ldots \ldots$. & 170 & adsky .. & 191 \\
\hline rel $\ldots \ldots \ldots \ldots$ & 71 & (25) & 177 \\
\hline$\ldots \ldots \ldots 192$ & 193 & $1 \ldots \ldots \quad 218$ & 229 \\
\hline$\ldots \ldots \ldots \quad 117$ & 291 & Zwaardemaker (Н.).. & 177 \\
\hline . & 180 & & \\
\hline
\end{tabular}

